\title{
MSTS
}

\section{Multiphase Subsurface \\ Transport Simulator User's Guide and Reference}

W. E. Nichols

M. D. White

May 1993

Prepared for the U.S. Department of Energy Office of Civilian Radioactive Waste Management under Contract DE-AC06-76RLO 1830

Pacific Northwest Laboratory Operated for the U.S. Department of Energy by Battelle Memorial Institute 


\title{
DISCLAIMER
}

This report was prepared as an account of work sponsored by an agency of the United States Government. Neither the United States Government nor any agency thereof, nor Battelle Memorial Institute, nor any of their employees, makes any warranty, expressed or implied, or assumes any legal liability or responsibility for the accuracy, completeness, or usefulness of any information, apparatus, product, or process disclosed, or represents that its use would not infringe privately owned rights. Reference herein to any specific commercial product, process, or service by trade name, trademark, manufacturer, or otherwise does not necessarily constitute or imply its endorsement, recommendation, or favoring by the United States Government or any agency thereof, or Battelle Memorial Institute. The views and opinions of authors expressed herein do not necessarily state or reflect those of the United States Government or any agency thereof.

\author{
PACIFIC NORTHWEST LABORATORY \\ operated by \\ BATTELLE MEMORIAL INSTITUTE \\ for the \\ UNITED STATES DEPARTMENT OF ENERGY \\ under Contract DE-ACO6-76RLO 1830
}

Printed in the United States of America

Available to DOE and DOE contractors from the

Office of Scientific and Technical Information, P.O. Box 62, Oak Ridge, TN 37831; prices available from (615) 576-8401. FTS 626-8401.

Available to the public from the National Technical Information Service,

U.S. Department of Commerce, 5285 Port Royal Rd., Springfield, VA 22161. 


\title{
MSTS
}

\section{MULTIPHASE SUBSURFACE TRANSPORT SIMULATOR USER'S GUIDE AND REFERENCE}

\author{
W. E. Nichols
}

M. D. White

May 1993

Prepared for the U.S. Department of Energy,

Office of Civilian Radioactive Waste Management under Contract DE-AC06-76RLO 1830

Pacific Northwest Laboratory Richland, Washington 99352 


\section{COPYRIGHT AND TRADEMARK ACKNOWLEDGMENTS}

MSTS- Developed by Battelle Memorial Institute, Pacific Northwest Laboratory.

MSTS Graphical Input- Developed by Battelle Memorial Institute, Pacific Northwest Laboratory. Portions $\mathbb{C}^{1989,}$ 1990, 1991 Silicon Beach Software ${ }^{\circledR}$, Inc., A Subsidiary of Aldus Corporation.

This application was created using Aldus SuperCard ${ }^{\otimes}$, from Silicon Beach Software, Inc., A Subsidiary of Aldus Corporation.

AIX is a registered trademark of International Business Machines Corporation.

Apple and Macintosh are registered trademarks of Apple Computer, Inc.

HP is a registered trademark of Hewlett Packard, Inc.

IBM is a registered trademark of International Business Machines Corporation.

SuperCard is a registered trademark of Silicon Beach Software, Inc.

Sun is a registered trademark of Sun Microsystems, Inc.

UNIX is a registered trademark of AT\&T Information Systems. 


\section{Preface}

This User's Guide and Reference is a software document written to provide instructions and a detailed reference necessary to use the Multiphase Subsurface Transport Simulator (MSTS) and a closely associated utility, the MSTS Graphical Input. The design architecture and specific computational algorithms that compose MSTS are delineated in a companion document, the MSTS Theory Manual.

It is assumed in writing this User's Guide and Reference that the user of this code has received advanced training in hydrogeology, heat transfer, and thermodynamics. It is also assumed that users are familiar with the computing environment in which they will compile and use the MSTS code.

Machine requirements for the MSTS code are dependent on the nature of the problem to be solved. The MSTS code is not machine-dependent, and with essentially minor changes (detailed in this User's Guide and Reference), the MSTS code may be compiled and run on many different computer environments using a FORTRAN 77 compiler. Naturally, the speed with which problems are solved depends on the capacity and speed of the computing platform and the size of the problem. Small one-dimensional problems may be solved quickly on a desktop computer, but large, complex two- or three-dimensional multiphase problems may be practically tackled only using a workstation or mainframe. The MSTS Graphical Input may be used only with a Macintosh computer. 
. 


\section{Summary}

This User's Guide and Reference provides information and instructions on the use of the Multiphase Subsurface Transport Simulator (MSTS) code and the associated MSTS Graphical Input. The MSTS code is used to simulate water flow, air flow, heat transfer, and dilute species mass transport in variably saturated geologic media for one, two, or three dimensions using an integrated finite-difference numerical scheme. Any or all of these processes may be simulated in a fully coupled manner. MSTS is a two-phase, two-component code with secondary processes that include binary diffusion and vapor pressure lowering. The geologic media may be homogeneous or heterogeneous, isotropic or anisotropic, and unfractured or highly fractured. A problem geometry may be described by either Cartesian or cylindrical coordinates. MSTS is written in FORTRAN 77, following the American National Standards Institute (ANSI) standards, and is machine-independent with the exception of some time and date calls required for quality control (provisions are made in the code for relatively easy adaption to a number of machines for these calls).

KEYWORDS: multiphase, ground-water flow, multiple phase, variably saturated, unsaturated, finite difference, numerical modeling, Newton-Raphson iteration, equivalent-continuum model. 


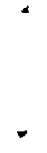




\section{Acknowledgments}

Linwood E. Peele is acknowledged for his contributions in the form of "quick tour" testing and assistance with sample problem preparation for this document. Mr. Peele provided this contribution during his Associated Western Universities EMCOM (Employment Career Opportunities for Minorities) appointment to Pacific Northwest Laboratory. 


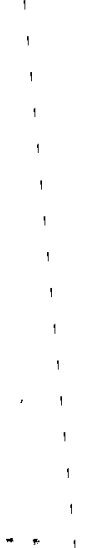




\section{Glossary of Symbols}

Roman Symbols

\begin{tabular}{|c|c|c|c|}
\hline Symbol & Description & Dimensions & SI(a) Units \\
\hline$A, A_{\ell}, A_{8}$ & function curve-fitting parameters & - & - \\
\hline$B, B_{\ell}, B_{g}$ & function curve-fitting parameters & - & - \\
\hline$c_{s}$ & $\begin{array}{l}\text { specific heat capacity of solid } \\
\text { phase }\end{array}$ & $\mathrm{L} 2 \mathrm{t}-2 \mathrm{~T}-1$ & $\mathrm{~J} / \mathrm{kg} \mathrm{K}$ \\
\hline$C, C_{\ell}, C_{g}$ & $\begin{array}{l}\text { species concentration (per total } \\
\text { volume, per aqueous volume, per } \\
\text { gas volume) }\end{array}$ & mol L-3 & $\mathrm{mol} / \mathrm{m}^{3}$ \\
\hline $\mathrm{Cr}$ & Courant Number & - & - \\
\hline$D$ & diffusion conductance & $\operatorname{mol~L-2~t-1}$ & $\mathrm{mol} / \mathrm{m}^{2} \mathrm{~s}$ \\
\hline$D_{g}^{a w}$ & air water-vapor binary diffusivity & $\mathrm{L}^{2} \mathrm{t}^{-1}$ & $\mathrm{~m}^{2} / \mathrm{s}$ \\
\hline$\tilde{D}_{h,}$ & $\begin{array}{l}\text { species hydraulic-dispersion } \\
\text { coefficient (aqueous) }\end{array}$ & $\mathrm{L}^{2} \mathrm{t}^{-1}$ & $\mathrm{~m}^{2} / \mathrm{s}$ \\
\hline$D_{d,}, D_{d}$ & $\begin{array}{l}\text { species diffusion coefficient } \\
\text { (aqueous, gas) }\end{array}$ & $\mathrm{L} 2 \mathrm{t}-1$ & $\mathrm{~m}^{2} / \mathrm{s}$ \\
\hline$g$ & acceleration of gravity & $\mathrm{Lt}^{\mathrm{t}-2}$ & $\mathrm{~m} / \mathrm{s}^{2}$ \\
\hline$h_{\ell}, h_{g}$ & enthalpy (aqueous, gas) & $\mathrm{L}^{2} \mathrm{t}^{-2}$ & $\mathrm{~J} / \mathrm{kg}$ \\
\hline$h^{w}, h^{a}$ & enthalpy (water, air) & $\mathrm{L}^{2} \mathrm{t} \cdot 2$ & $\mathrm{~J} / \mathrm{kg}$ \\
\hline$H_{T}, H_{E}, H_{p}$ & $\begin{array}{l}\text { hydraulic head (total, elevational, } \\
\text { pressure) }\end{array}$ & $\mathrm{L}$ & $\mathrm{m}$ \\
\hline$\tilde{k}$ & intrinsic permeability tensor & $\mathrm{L}^{2}$ & $\mathrm{~m}^{2}$ \\
\hline$k_{r \ell}, k_{r g}$ & $\begin{array}{l}\text { relative permeability (aqueous, } \\
\text { gas) }\end{array}$ & - & - \\
\hline$k_{r \ell_{b}}, k_{r \ell_{f}}, k_{r \ell_{m}}$ & $\begin{array}{l}\text { aqueous relative permeability } \\
\text { (bulk, fracture, matrix) }\end{array}$ & - & - \\
\hline
\end{tabular}

(a) Système Intemational 
Roman Symbols (Contd)

\begin{tabular}{|c|c|c|c|}
\hline Symbol & Description & Dimensions & SI Units \\
\hline$\tilde{k}_{e}$ & $\begin{array}{l}\text { effective thermal conductivity } \\
\text { tensor }\end{array}$ & $M L_{t}-2 T-1$ & $\mathrm{~W} / \mathrm{m} \mathrm{K}$ \\
\hline$K_{d}$ & $\begin{array}{l}\text { partition coefficient (solid- } \\
\text { aqueous systems) }\end{array}$ & - & - \\
\hline$K_{\ell g}$ & $\begin{array}{l}\text { partition coefficient (aqueous-gas } \\
\text { systems) }\end{array}$ & - & - \\
\hline$m$ & $\begin{array}{l}\text { moisture-retention curve-fitting } \\
\text { parameter }\end{array}$ & - & - \\
\hline$m_{f}, m_{m}$ & $\begin{array}{l}\text { moisture-retention curve-fitting } \\
\text { parameter (fracture, matrix) }\end{array}$ & - & - \\
\hline$m_{\ell}^{w}, m_{g}^{w}$ & water mass (aqueous, gas phases) & $\mathbf{M}$ & $\mathrm{kg}$ \\
\hline$\dot{m}^{w}, \dot{m}^{a}$ & $\begin{array}{l}\text { mass source rate per volume } \\
\text { (water, air) }\end{array}$ & $\mathrm{M} \mathrm{L}^{-3} \mathrm{t}^{-1}$ & $\mathrm{~kg} / \mathrm{m}^{3} \mathrm{~s}$ \\
\hline$\dot{m}_{\ell}, \dot{m}_{8}$ & mass source rate (aqueous, gas) & $\mathrm{Mt}^{-1}$ & $\mathrm{~kg} / \mathrm{s}$ \\
\hline$n$ & $\begin{array}{l}\text { moisture-retention curve-fitting } \\
\text { parameter }\end{array}$ & - & - \\
\hline$n_{f}, n_{m}$ & $\begin{array}{l}\text { moisture-retention curve-fitting } \\
\text { parameter (fracture, matrix) }\end{array}$ & - & - \\
\hline$n_{D}, n_{E}, n_{T}$ & porosity (diffusive, effective, total) & - & - \\
\hline${ }^{n} D_{j}, n_{E_{j}}, n_{T_{j}}$ & $\begin{array}{l}\text { fracture porosity (diffusive, } \\
\text { effective, total) }\end{array}$ & - & - \\
\hline$n_{D_{m}}, n_{E_{m}}, n_{T_{m}}$ & $\begin{array}{l}\text { matrix porosity (diffusive, } \\
\text { effective, total) }\end{array}$ & - & - \\
\hline$P_{\ell}, P_{g}$ & pressure (liquid, gas) & $M L^{-1} \mathfrak{t}^{-2}$ & $\mathrm{~Pa}$ \\
\hline$P_{\text {cap }}$ & capillary pressure & $M L^{-1} t^{-2}$ & $\mathrm{~Pa}$ \\
\hline Pe & Peclet Number & - & - \\
\hline$q$ & thermal energy & $M L^{2} t^{-2}$ & $\mathrm{~J}$ \\
\hline
\end{tabular}


Roman Symbols (Contd)

\begin{tabular}{|c|c|c|c|}
\hline Symbol & Description & Dimensions & SI Units \\
\hline$\dot{q}$ & $\begin{array}{l}\text { thermal energy source power } \\
\text { density }\end{array}$ & $M L^{2} t^{-3}$ & $\mathrm{~W} / \mathrm{m}^{3}$ \\
\hline$\dot{Q}_{e}$ & thermal energy flux rate & $\mathbf{M t}^{t^{-3}}$ & $\mathrm{~W} / \mathrm{m}^{2}$ \\
\hline$\dot{Q}_{c}$ & species flux rate & $\mathrm{mol} \mathrm{L}-2 \mathrm{t}^{-1}$ & $\mathrm{~mol} / \mathrm{m}^{2} \mathrm{~s}$ \\
\hline$\dot{Q}_{\ell}, \dot{Q}_{g}$ & $\begin{array}{l}\text { mass flux rate (aqueous, gas } \\
\text { phases) }\end{array}$ & $M L^{-2} t^{-1}$ & $\mathrm{~kg} / \mathrm{m}^{2} \mathrm{~s}$ \\
\hline$r$ & $\begin{array}{l}\text { radial coordinate (cylindrical } \\
\text { coordinate systems) }\end{array}$ & radians & radians \\
\hline$r_{0}$ & $\begin{array}{l}\text { inner radius of model domain } \\
\text { (cylindrical coordinate systems) }\end{array}$ & radians & radians \\
\hline$R^{w}, R^{a}$ & ideal gas constant (water, air) & $\mathrm{L}^{2} \mathrm{t}-2 \mathrm{~T}-1$ & $\mathrm{~J} / \mathrm{kg} \mathrm{K}$ \\
\hline$\dot{R}_{c}$ & species decay rate & $t-1$ & $1 / \mathrm{s}$ \\
\hline$\dot{s}_{c}$ & species source (mass basis) & $\mathrm{mol} \mathrm{L}-3 \mathrm{t}^{-1}$ & $\mathrm{~mol} / \mathrm{s}$ \\
\hline$\dot{s}_{c, v}$ & $\begin{array}{l}\text { species source (concentration } \\
\text { basis) }\end{array}$ & $\operatorname{mol~L-3~t-1~}$ & $\mathrm{mol} / \mathrm{m}^{3} \mathrm{~s}$ \\
\hline$s_{\ell}, s_{g}$ & saturation (aqueous, gas) & - & - \\
\hline$s_{\ell_{b}} s_{\ell_{j}}, s_{\ell_{m}}$ & $\begin{array}{l}\text { aqueous saturation (bulk, fracture, } \\
\text { matrix) }\end{array}$ & - & - \\
\hline$s_{i}^{*}$ & effective aqueous saturation & - & - \\
\hline$s_{\ell r}$ & residual aqueous saturation & - & - \\
\hline$s_{\ell r_{f}} s_{\ell r_{m}}$ & $\begin{array}{l}\text { residual aqueous saturation } \\
\text { (fracture, matrix) }\end{array}$ & - & - \\
\hline$s_{s r}$ & entrapped air saturation & - & - \\
\hline$S_{s}$ & coefficient of specific storage & $L^{-1}$ & $1 / \mathrm{m}$ \\
\hline$t$ & time & $\mathrm{t}$ & s \\
\hline$T$ & temperature & $\mathrm{T}$ & ${ }^{\circ} \mathrm{C}$ or $\mathrm{K}$ \\
\hline
\end{tabular}


Roman Symbols (Contd)

\begin{tabular}{|c|c|c|c|}
\hline Symbol & Description & Dimensions & SI Units \\
\hline$u_{s}$ & solid-phase internal energy & $\mathrm{L}^{2} \mathrm{t}-2$ & $\mathrm{~J} / \mathrm{kg}$ \\
\hline$u_{8}^{w}, u_{8}^{a}$ & $\begin{array}{l}\text { gas-phase internal energy (water, } \\
\text { air) }\end{array}$ & $\mathrm{L}^{2} \mathrm{t}-2$ & $\mathrm{~J} / \mathrm{kg}$ \\
\hline$u_{\ell}^{w}, u_{\ell}^{a}$ & $\begin{array}{l}\text { aqueous-phase internal energy } \\
\text { (water, air) }\end{array}$ & $\mathrm{L}^{2} \mathrm{t}-2$ & $\mathrm{~J} / \mathrm{kg}$ \\
\hline$u$ & east-west Darcy velocity & $\mathrm{Lt}^{-1}$ & m \\
\hline$V$ & volume & $\mathrm{L}^{3}$ & $\mathrm{~m}^{3}$ \\
\hline $\mathbf{V}_{\ell}, \mathbf{V}_{g}$ & phase velocity (aqueous, gas) & $\mathrm{Lt}^{-1}$ & $\mathrm{~m} / \mathrm{s}$ \\
\hline$v$ & south-north Darcy velocity & $\mathrm{Lt}^{-1}$ & $\mathrm{~m} / \mathrm{s}$ \\
\hline$w$ & bottom-top Darcy velocity & $\mathrm{Lt}^{-1}$ & $\mathrm{~m} / \mathrm{s}$ \\
\hline$x$ & $\begin{array}{l}\text { west-east coordinate (Cartesian } \\
\text { coordinate systems) }\end{array}$ & $\mathrm{L}$ & $\mathrm{m}$ \\
\hline$x_{g}^{w}, x_{g}^{a}$ & $\begin{array}{l}\text { gas-phase mass fraction (water, } \\
\text { air) }\end{array}$ & - & - \\
\hline$x_{p}^{w}, x_{\ell}^{a}$ & $\begin{array}{l}\text { aqueous-phase mass fraction } \\
\text { (water, air) }\end{array}$ & - & - \\
\hline$y$ & $\begin{array}{l}\text { south-north coordinate (Cartesian } \\
\text { coordinate systems) }\end{array}$ & $\mathbf{L}$ & $\mathrm{m}$ \\
\hline$z$ & $\begin{array}{l}\text { bottom-top coordinate (Cartesian } \\
\text { and cylindrical coordinate } \\
\text { systems) }\end{array}$ & $\mathbf{L}$ & $\mathrm{m}$ \\
\hline$\hat{z}$ & elevational gradient & $\mathrm{L} \mathrm{L}^{-1}$ & $\mathrm{~m} / \mathrm{m}$ \\
\hline$z^{*}$ & datum elevation & $\mathrm{L}$ & $\mathrm{m}$ \\
\hline
\end{tabular}


Greek Symbols

\begin{tabular}{|c|c|c|c|}
\hline Symbol & Description & Dimensions & SI Units \\
\hline$\alpha$ & $\begin{array}{l}\text { moisture-retention curve-fitting } \\
\text { parameter }\end{array}$ & $L^{-1}$ & $1 / \mathrm{m}$ \\
\hline$\alpha_{f}, \alpha_{m}$ & $\begin{array}{l}\text { moisture-retention curve-fitting } \\
\text { parameter (fracture, matrix) }\end{array}$ & $L-1$ & $1 / \mathrm{m}$ \\
\hline$\beta$ & $\begin{array}{l}\text { moisture-retention curve-fitting } \\
\text { parameter }\end{array}$ & - & - \\
\hline$\eta_{D}$ & total water content & $\mathrm{L}^{3} \mathrm{~L}^{-3}$ & $\mathrm{~m}^{3} / \mathrm{m}^{3}$ \\
\hline$\theta$ & volumetric moisture content & $\mathrm{L}^{3} \mathrm{~L}^{-3}$ & $\mathrm{~m}^{3} / \mathrm{m}^{3}$ \\
\hline$\theta_{r}$ & residual moisture content & $\mathrm{L}^{3} \mathrm{~L}^{-3}$ & $\mathrm{~m}^{3} / \mathrm{m}^{3}$ \\
\hline$\theta_{s}$ & saturated moisture content & $\mathrm{L}^{3} \mathrm{~L}^{-3}$ & $\mathrm{~m}^{3} / \mathrm{m}^{3}$ \\
\hline$\theta$ & $\begin{array}{l}\text { angular coordinate (cylindrical } \\
\text { coordinate systems) }\end{array}$ & radians & radians \\
\hline$\mu_{g}^{w}, \mu_{g}^{a}$ & $\begin{array}{l}\text { gas-phase component viscosity } \\
\text { (water, air) }\end{array}$ & $M L^{-1} t^{-2}$ & Pas \\
\hline$\mu_{\ell}, \mu_{\mathrm{g}}$ & viscosity (aqueous, gas) & $\mathrm{ML}^{-1} \mathrm{t}^{-2}$ & Pas \\
\hline$\rho_{\ell}, \rho_{g}, \rho_{s}$ & mass density (aqueous, gas, solid) & $\mathrm{M} \mathrm{L}^{-3}$ & $\mathrm{~kg} / \mathrm{m}^{3}$ \\
\hline$\sigma^{a w}$ & air-water surface tension & $M t^{-2}$ & $\mathrm{~N} / \mathrm{m}$ \\
\hline$\tau_{\ell}, \tau_{g}$ & tortuosity (aqueous, gas) & - & - \\
\hline$\chi_{\ell}^{a}, \chi_{g}^{a}$ & mole fraction of air (aqueous, gas) & - & - \\
\hline$\psi$ & $\begin{array}{l}\text { tension head (negative pressure } \\
\text { head) }\end{array}$ & L & $\mathrm{m}$ \\
\hline$\psi^{*}$ & $\begin{array}{l}\text { minimum drainage capillary } \\
\text { pressure head }\end{array}$ & $\mathrm{L}$ & $\mathrm{m}$ \\
\hline
\end{tabular}




\section{Subscripts}

\begin{tabular}{|c|c|}
\hline Symbol & Description \\
\hline cap & capillary \\
\hline$D$ & diffusive \\
\hline$e$ & equivalent \\
\hline E & effective \\
\hline$f$ & fracture \\
\hline$g$ & gas phase \\
\hline$\ell$ & aqueous phase \\
\hline$r$ & residual \\
\hline$s$ & solid phase; saturation state \\
\hline $\mathbf{T}$ & total \\
\hline
\end{tabular}

\section{Superscripts, Overscripts}

\begin{tabular}{cl} 
Symbol & \multicolumn{1}{c}{ Description } \\
$\sim$ & tensor \\
$*$ & reference value; effective
\end{tabular}

\section{Mathematical Symbols}

\begin{tabular}{lll} 
Symbol & & \multicolumn{1}{c}{ Description } \\
\cline { 1 - 1 } $\boldsymbol{D}$ & & divergence operator \\
$\partial$ & & partial differential operator \\
() & & function operator \\
I 1 & & absolute value operator \\
$\ln (x)$ & & natural logarithm of variable $\mathrm{x}$
\end{tabular}




\section{Contents}

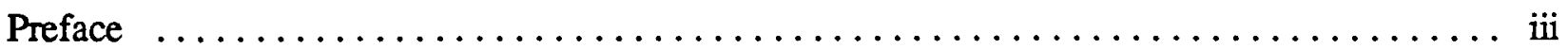

Summary $\ldots \ldots \ldots \ldots \ldots \ldots \ldots \ldots \ldots \ldots \ldots \ldots \ldots \ldots \ldots \ldots \ldots \ldots \ldots \ldots \ldots$

Acknowledgments $\ldots \ldots \ldots \ldots \ldots \ldots \ldots \ldots \ldots \ldots \ldots \ldots \ldots \ldots \ldots \ldots \ldots \ldots \ldots \ldots \ldots$ vii

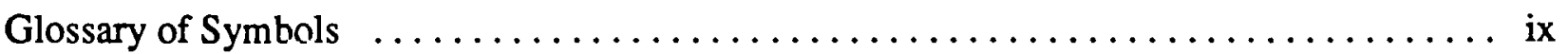

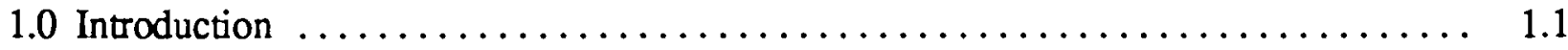

2.0 MSTS Capabilities Overview $\ldots \ldots \ldots \ldots \ldots \ldots \ldots \ldots \ldots \ldots \ldots \ldots \ldots \ldots \ldots \ldots \ldots \ldots$

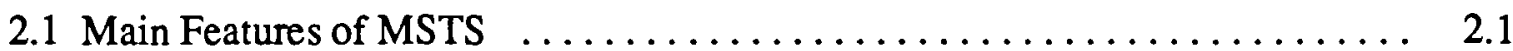

2.1.1 Spatial Dimensionality and Coordinate Systems $\ldots \ldots \ldots \ldots \ldots \ldots . .1$

2.1.2 Time and Space Dependence $\ldots \ldots \ldots \ldots \ldots \ldots \ldots \ldots \ldots \ldots \ldots \ldots \ldots$

2.1 .3 Equation Coupling $\ldots \ldots \ldots \ldots \ldots \ldots \ldots \ldots \ldots \ldots \ldots \ldots \ldots \ldots .2$

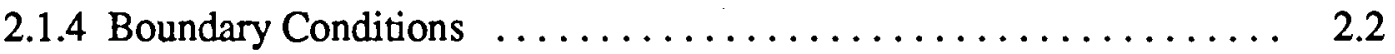

2.1.5 Solution Methods for Governing Equations $\ldots \ldots \ldots \ldots \ldots \ldots . \ldots . \ldots . \ldots$

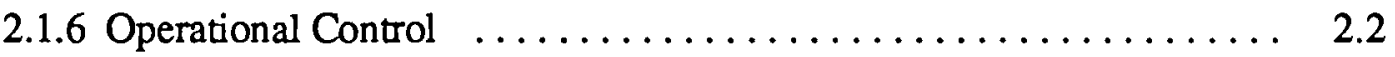

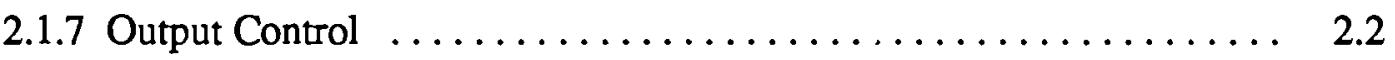

2.1.8 Variable Saturation $\ldots \ldots \ldots \ldots \ldots \ldots \ldots \ldots \ldots \ldots \ldots \ldots$

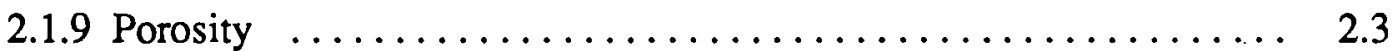

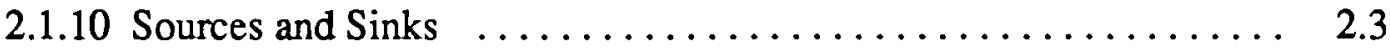

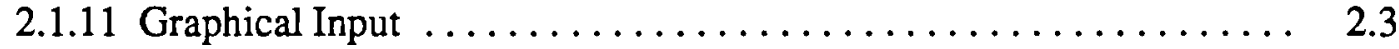

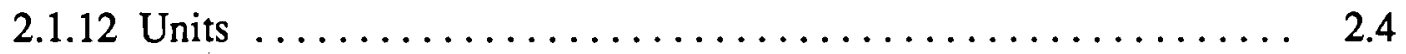

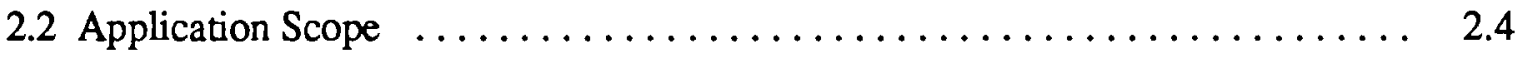

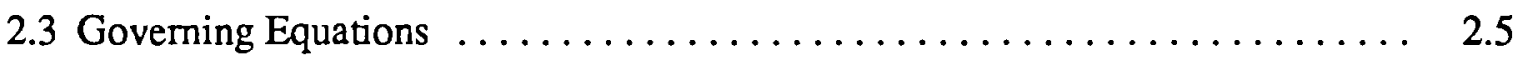

2.3.1 Water Mass Conservation Equation $\ldots \ldots \ldots \ldots \ldots \ldots \ldots \ldots \ldots$

2.3.2 Air Mass Conservation Equation $\ldots \ldots \ldots \ldots \ldots \ldots \ldots \ldots . \ldots \ldots$

2.3.3 Thermal Energy Conservation Equation $\ldots \ldots \ldots \ldots \ldots \ldots \ldots \ldots$

2.3.4 Species Mass Conservation Equation $\ldots \ldots \ldots \ldots \ldots \ldots \ldots \ldots$

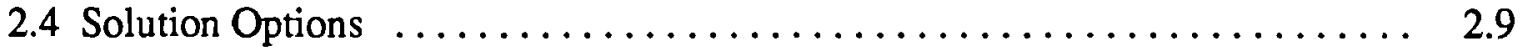

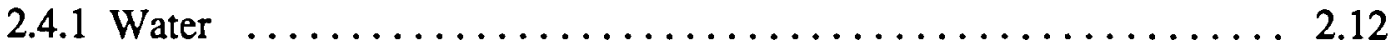

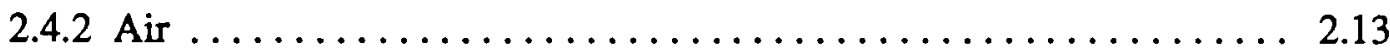

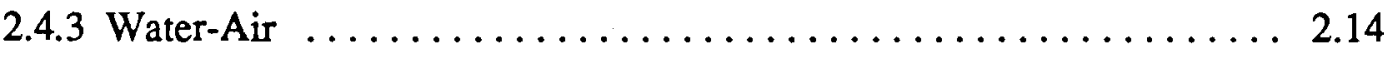

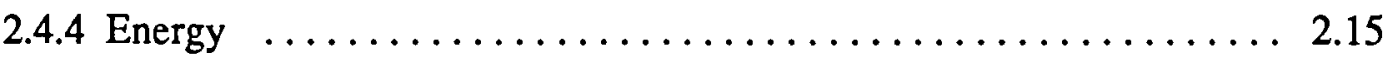

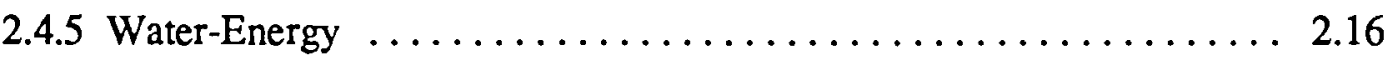

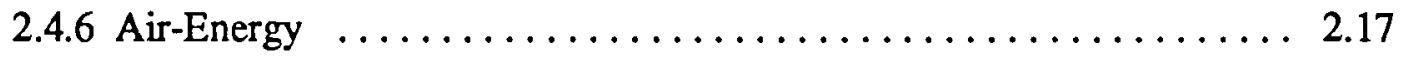




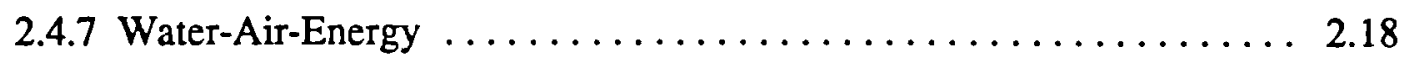

3.0 Graphical Input Overview $\ldots \ldots \ldots \ldots \ldots \ldots \ldots \ldots \ldots \ldots \ldots \ldots \ldots \ldots \ldots \ldots \ldots \ldots \ldots .1$

4.0 File Management $\ldots \ldots \ldots \ldots \ldots \ldots \ldots \ldots \ldots \ldots \ldots \ldots \ldots \ldots \ldots \ldots \ldots \ldots, 4.1$

4.1 MSTS Input and Output Files $\ldots \ldots \ldots \ldots \ldots \ldots \ldots \ldots \ldots \ldots \ldots \ldots, 4.1$

4.2 MSTS Source Code Parameterization $\ldots \ldots \ldots \ldots \ldots \ldots \ldots \ldots \ldots . . .2$

4.3 MSTS Source Code Machine Specification ................... 4.4

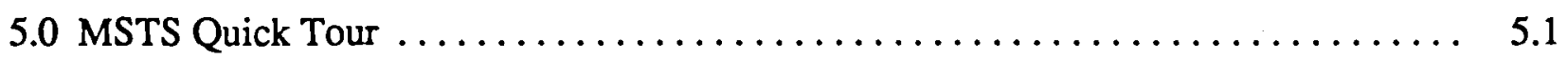

5.1 Installing MSTS on a Macintosh Computer $\ldots \ldots \ldots \ldots \ldots \ldots \ldots \ldots . .1$

5.2 Launching the MSTS Graphical Input $\ldots \ldots \ldots \ldots \ldots \ldots \ldots \ldots \ldots \ldots .6 \ldots \ldots$

5.3 Creating a New Input File $\ldots \ldots \ldots \ldots \ldots \ldots \ldots \ldots \ldots \ldots \ldots \ldots \ldots \ldots \ldots \ldots .1$

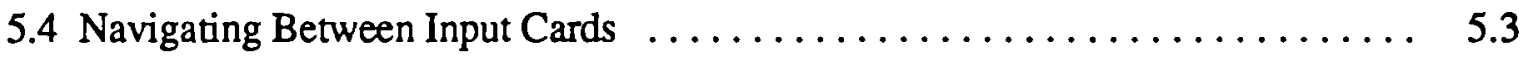

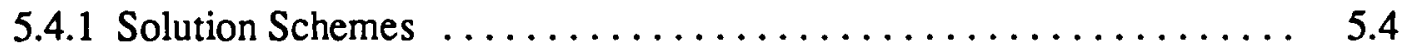

5.4 .2 Numerical Control $\ldots \ldots \ldots \ldots \ldots \ldots \ldots \ldots \ldots \ldots \ldots . . \ldots \ldots$

5.4 .3 Grid Geometry $\ldots \ldots \ldots \ldots \ldots \ldots \ldots \ldots \ldots \ldots \ldots \ldots \ldots \ldots \ldots \ldots \ldots .7$

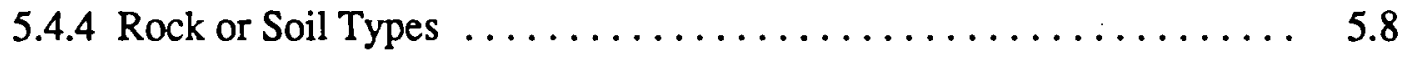

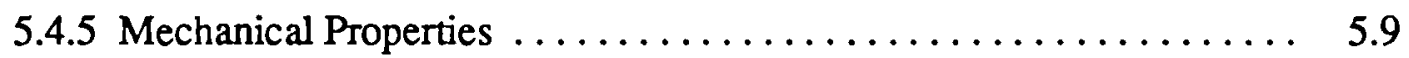

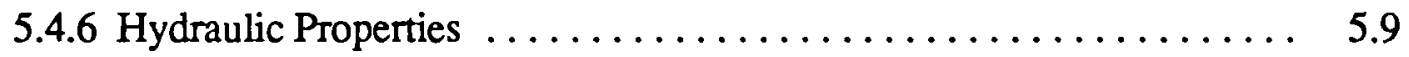

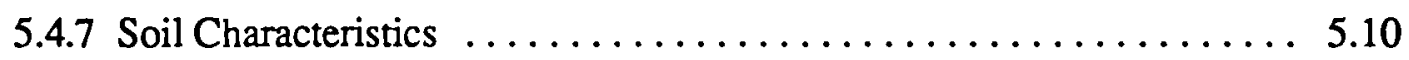

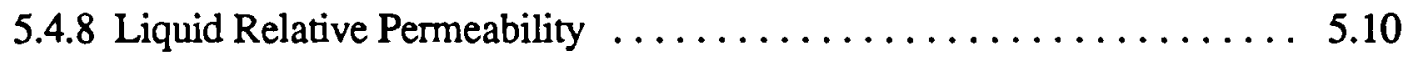

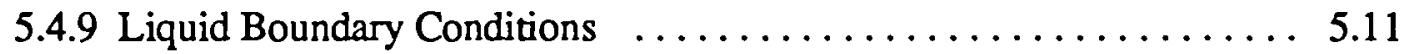

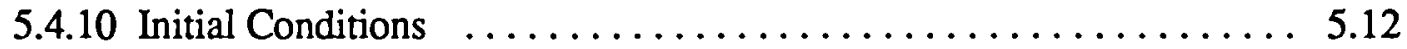

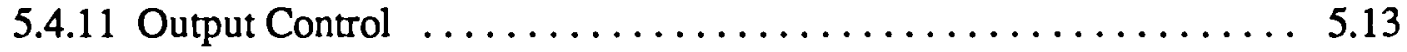

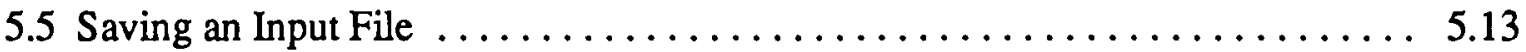

5.6 Parameterization and Machine Specification $\ldots \ldots \ldots \ldots \ldots \ldots \ldots \ldots . . \ldots \ldots$

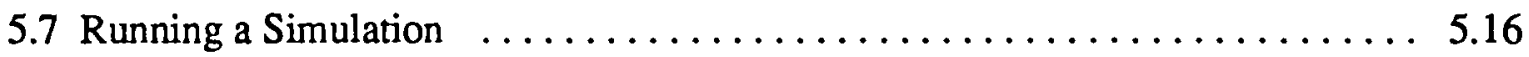

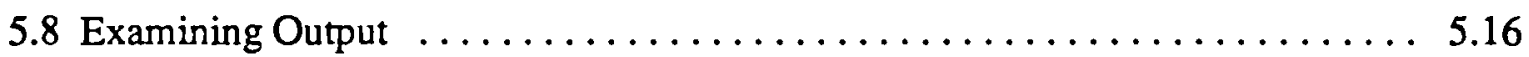

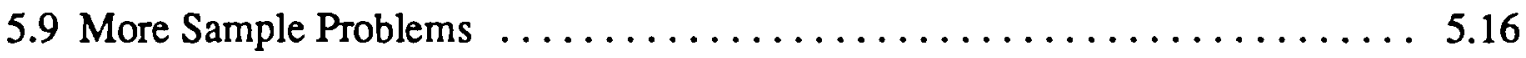

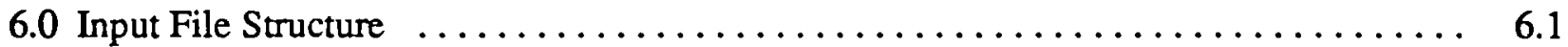

6.1 Simulation Documentation and Control $\ldots \ldots \ldots \ldots \ldots \ldots \ldots \ldots \ldots .6 .5$

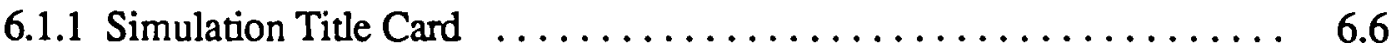

6.1 .2 Solution Schemes Card $\ldots \ldots \ldots \ldots \ldots \ldots \ldots \ldots \ldots \ldots . \ldots \ldots$

6.1 .3 Numerical Control Card $\ldots \ldots \ldots \ldots \ldots \ldots \ldots \ldots \ldots \ldots . \ldots \ldots .11$

6.2 Computational Domain $\ldots \ldots \ldots \ldots \ldots \ldots \ldots \ldots \ldots \ldots \ldots \ldots \ldots \ldots \ldots \ldots \ldots \ldots .13$

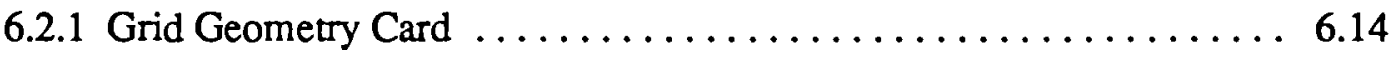


6.2.2 Inactive Nodes Card

6.2.3 Rock or Soil Types Card

6.3 Physical Properties $\ldots \ldots \ldots \ldots \ldots \ldots \ldots \ldots \ldots \ldots \ldots \ldots . \ldots \ldots . .22$

6.3.1 Mechanical Properties Card $\ldots \ldots \ldots \ldots \ldots \ldots \ldots \ldots \ldots . \ldots . \ldots \ldots \ldots . \ldots .23$

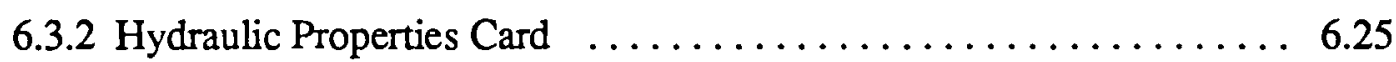

6.3.3 Thermal Properties Card $\ldots \ldots \ldots \ldots \ldots \ldots \ldots \ldots \ldots \ldots . \ldots .27$

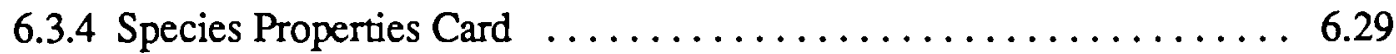

6.3.5 Soil Characteristics Card $\ldots \ldots \ldots \ldots \ldots \ldots \ldots \ldots \ldots \ldots . \ldots . \ldots \ldots \ldots$

6.3.6 Liquid Relative Permeability Card ................... 6.35

6.3.7 Gas Relative Permeability Card ................... 6.40

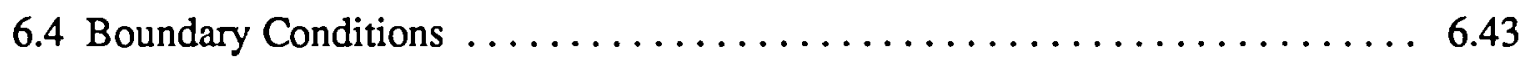

6.4 .1 Liquid Boundary Conditions Card $\ldots \ldots \ldots \ldots \ldots \ldots \ldots . \ldots .44$

6.4 .2 Gas Boundary Conditions Card $\ldots \ldots \ldots \ldots \ldots \ldots \ldots \ldots .6 .47$

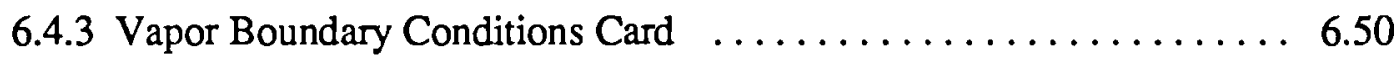

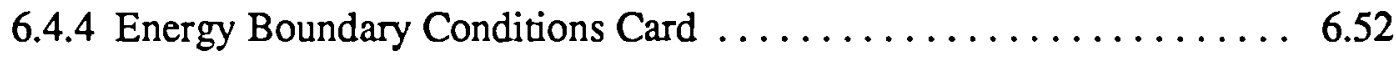

6.4.5 Species Boundary Conditions Card $\ldots \ldots \ldots \ldots \ldots \ldots \ldots \ldots . \ldots . . .65$

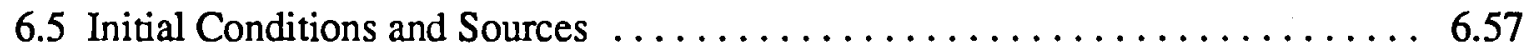

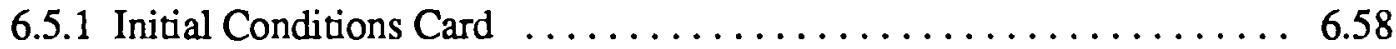

6.5 .2 Sources and Sinks Card $\ldots \ldots \ldots \ldots \ldots \ldots \ldots \ldots \ldots . \ldots \ldots 1$

6.6 Output Control ........................................6.69

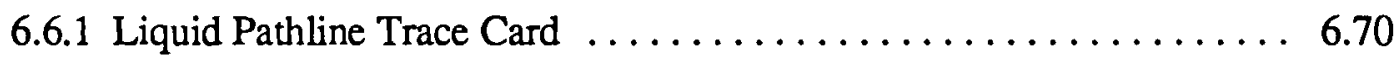

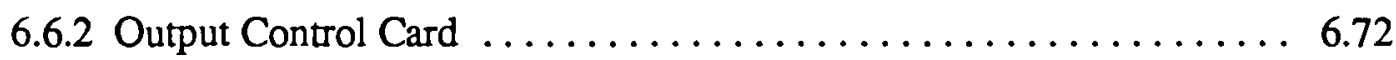

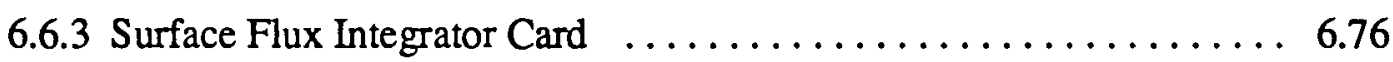

7.0 Water-Retention and Relative-Permeability Functions $\ldots \ldots \ldots \ldots \ldots \ldots \ldots . \ldots .1$

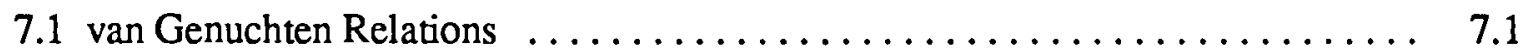

7.2 Brooks and Corey Relations $\ldots \ldots \ldots \ldots \ldots \ldots \ldots \ldots \ldots \ldots \ldots . .2$

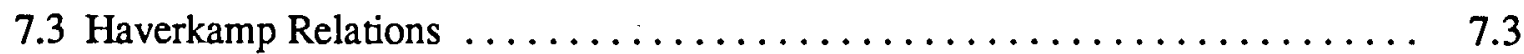

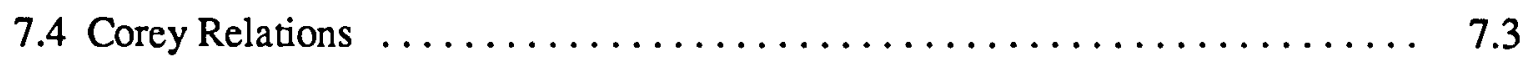

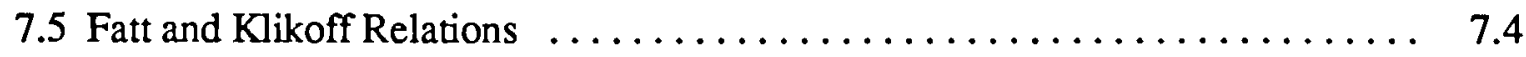

7.6 Leverett Relations .................................... 7.4

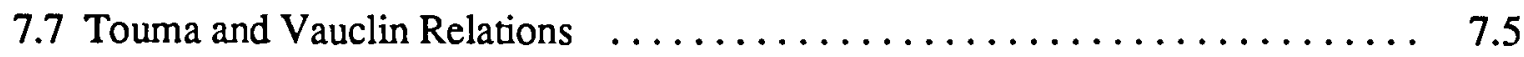

7.8 Dual-Porosity Model With van Genuchten Relations $\ldots \ldots \ldots \ldots \ldots \ldots . .5$

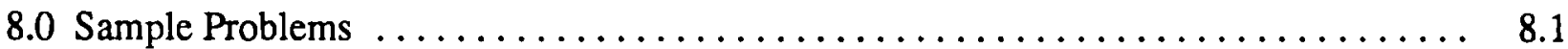

8.1 Single-Node Evaporation $\ldots \ldots \ldots \ldots \ldots \ldots \ldots \ldots \ldots \ldots \ldots \ldots, 8.3$ 
8.1.1 Problem Domain and Input Parameters $\ldots \ldots \ldots \ldots \ldots \ldots \ldots . \quad 8.3$

8.1 .2 Simulation Results $\ldots \ldots \ldots \ldots \ldots \ldots \ldots \ldots \ldots \ldots \ldots . \quad 8.3$

8.1.3 Mass Balance Check ......................... 8.3

8.2 Single-Node Condensation $\ldots \ldots \ldots \ldots \ldots \ldots \ldots \ldots \ldots \ldots \ldots . .17$

8.2.1 Problem Domain and Input Parameters $\ldots \ldots \ldots \ldots \ldots \ldots \ldots . .17$

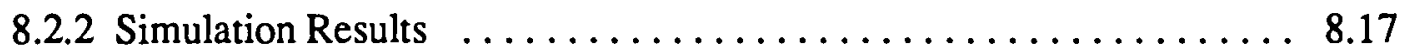

8.2 .3 Mass Balance Check ......................... 8.17

8.3 Flow from Hot Two-Phase Conditions $\ldots \ldots \ldots \ldots \ldots \ldots \ldots \ldots \ldots . \ldots .22$

8.3.1 Problem Domain and Input Parameters $\ldots \ldots \ldots \ldots \ldots \ldots \ldots . . \ldots .22$

8.3 .2 Energy Balance Check $\ldots \ldots \ldots \ldots \ldots \ldots \ldots \ldots \ldots \ldots . \ldots \ldots \ldots \ldots \ldots \ldots .22$

8.4 Philip's Vertical Infiltration Problem $\ldots \ldots \ldots \ldots \ldots \ldots \ldots \ldots \ldots \ldots . \ldots .27$

8.4 .1 Purpose $\ldots \ldots \ldots \ldots \ldots \ldots \ldots \ldots \ldots \ldots \ldots \ldots \ldots \ldots . \ldots .27$

8.4.2 Model Domain and Input Parameters $\ldots \ldots \ldots \ldots \ldots \ldots \ldots . .27$

8.4.3 Simulation Results and Comparison to Semi-Analytic Solution . . . . 8.29

8.5 Two-Dimensional Infiltration . . . . . . . . . . . . . . . . . . . 8.35

8.5.1 Problem Domain and Input Parameters $\ldots \ldots \ldots \ldots \ldots \ldots \ldots .6 .35$

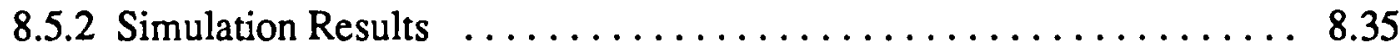

8.6 One-Dimensional Two-Phase Infiltration $\ldots \ldots \ldots \ldots \ldots \ldots \ldots \ldots \ldots .41$

8.6.1 Problem Domain and Input Parameters $\ldots \ldots \ldots \ldots \ldots \ldots \ldots . \ldots .41$

8.6 .2 Simulation Results $\ldots \ldots \ldots \ldots \ldots \ldots \ldots \ldots \ldots \ldots \ldots .8 .42$

8.7 One-Dimensional Heat Pipe $\ldots \ldots \ldots \ldots \ldots \ldots \ldots \ldots \ldots \ldots \ldots . \ldots . .54$

8.7.1 Problem Parameters and Input File $\ldots \ldots \ldots \ldots \ldots \ldots \ldots \ldots .6 .54$

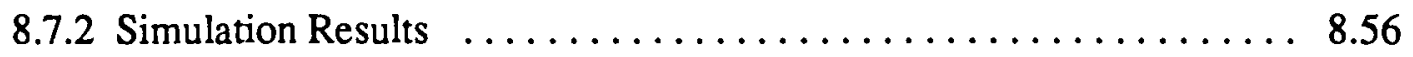

8.8 Unsaturated Transport $\ldots \ldots \ldots \ldots \ldots \ldots \ldots \ldots \ldots \ldots \ldots \ldots \ldots .6 .62$

8.8.1 Problem Domain and Input Parameters $\ldots \ldots \ldots \ldots \ldots \ldots \ldots . \ldots .62$

8.8.2 Semi-Analytic Solution $\ldots \ldots \ldots \ldots \ldots \ldots \ldots \ldots \ldots \ldots \ldots . \ldots \ldots \ldots$

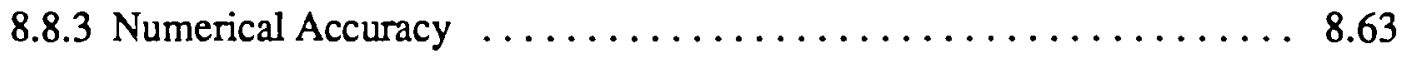

8.8.4 Simulation Results and Comparison to Semi-Analytic Solution . . . . 8.64

9.0 References $\ldots \ldots \ldots \ldots \ldots \ldots \ldots \ldots \ldots \ldots \ldots \ldots \ldots \ldots \ldots \ldots . . .6 .1$ 


\section{Figures}

5.1. Information Window from MSTS Graphical Input $\ldots \ldots \ldots \ldots \ldots \ldots \ldots \ldots$

5.2. Menu Bar from MSTS Graphical Input $\ldots \ldots \ldots \ldots \ldots \ldots \ldots \ldots \ldots \ldots \ldots \ldots$

5.3. File Dialog Box from MSTS Graphical Input $\ldots \ldots \ldots \ldots \ldots \ldots \ldots \ldots \ldots$

5.4. Initialization Prompt for New Input Files $\ldots \ldots \ldots \ldots \ldots \ldots \ldots \ldots \ldots \ldots \ldots$

5.5. Main Menu Card from MSTS Graphical Input $\ldots \ldots \ldots \ldots \ldots \ldots \ldots \ldots \ldots$

5.6. Simulation Title Input Card Example $\ldots \ldots \ldots \ldots \ldots \ldots \ldots \ldots \ldots \ldots \ldots \ldots$

5.7. Solution Schemes Input Card Example $\ldots \ldots \ldots \ldots \ldots \ldots \ldots \ldots \ldots \ldots \ldots \ldots$

5.8. Grid Geometry Input Card Example $\ldots \ldots \ldots \ldots \ldots \ldots \ldots \ldots \ldots \ldots \ldots$

5.9. Rock List from MSTS Graphical Input $\ldots \ldots \ldots \ldots \ldots \ldots \ldots \ldots$

5.10. Example of MSTS Graphical Input Interactive Help Feature $\ldots \ldots \ldots \ldots \ldots . \ldots$

5.11. Output Control Input Card from MSTS Graphical Input $\ldots \ldots \ldots \ldots \ldots \ldots . \ldots$.

5.12. Example of the "Compute Parameters" Resource of the MSTS Graphical Input . . 5.15

6.1. Example Grid Nodes and Associated Grid Cells for Uniform Node Distribution . . 6.16

6.2. Example Grid Nodes and Associated Grid Cells for Nonuniform Node Distribution . 6.16

8.1. MSTS-Predicted Temperature and Liquid Saturation Variation with Time for the Single-Node-Evaporation Problem $\ldots \ldots \ldots \ldots \ldots \ldots \ldots \ldots \ldots \ldots \ldots$

8.2. MSTS-Predicted Temperature and Liquid Saturation Variation with Time for the Single-Node-Condensation Problem $\ldots \ldots \ldots \ldots \ldots \ldots \ldots \ldots \ldots \ldots . \ldots \ldots$

8.3. Comparison of MSTS Numerical Solutions to the Analytical Solution of the Philip's Vertical Infiltration Problem After 2 Hours of Infiltration . . . . . . . . . 8.30

8.4. Two-Dimensional Infiltration Problem Geometry, Discretization, and Boundary

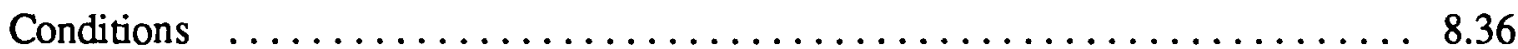

8.5. Functional Relationships Among Liquid Saturation, Liquid Pressure, and Liquid Relative Permeability for the Two-Dimensional Infiltration Problem $\ldots \ldots \ldots \ldots .36$

8.6. Capillary Pressure Profiles at 0.508 Day for Two-Dimensional Infiltration Problem . 8.37

8.7. Liquid Saturation Contours at 0.508 Day for Two-Dimensional Infiltration Problem . 8.37

8.8. Moisture-Retention and Relative-Permeability Functions for the One-Dimensional Two-Phase Infiltration Problem $\ldots \ldots \ldots \ldots \ldots \ldots \ldots \ldots \ldots \ldots \ldots \ldots . \ldots \ldots$

8.9. Water Content Profiles Predicted by MSTS for Ponded Infiltration in the OneDimensional, Two-Phase Infiltration Problem: Open Column $\ldots \ldots \ldots \ldots \ldots . . .43$

8.10. Water Content Profiles Predicted by MSTS for Ponded Infiltration in the OneDimensional, Two-Phase Infiltration Problem: Bounded Column $\ldots \ldots \ldots \ldots . .8 .44$ 
8.11. Schematic Describing the Heat Pipe Problem $\ldots \ldots \ldots \ldots \ldots \ldots \ldots \ldots \ldots . \ldots . . \ldots . \ldots$

8.12. Steady-State Temperature Profiles for Heat Pipe Problem .............. 8.57

8.13. Steady-State Saturation, Air Mole Fraction, and Advective/Total Ratio Profiles for Heat Pipe Problem $\ldots \ldots \ldots \ldots \ldots \ldots \ldots \ldots \ldots \ldots \ldots \ldots . \ldots . . .57$

8.14. Steady-State Heat Transport Modes for Heat Pipe Problem . . . . . . . . . . . 8.58

8.15. Dimensionless Solute Concentration Versus Distance at Various Times Using the Semi-Analytical Solution of Smiles et al. (1977) and MSTS Solutions With Two Spatial and Temporal Discretizations 


\section{Tables}

4.1. MSTS Input and Output File Name Convention $\ldots \ldots \ldots \ldots \ldots \ldots \ldots \ldots \ldots .2$

4.2. MSTS Parameters $\ldots \ldots \ldots \ldots \ldots \ldots \ldots \ldots \ldots \ldots \ldots \ldots \ldots \ldots \ldots \ldots . . \ldots \ldots$

4.3. MSTS-Supported Machines and Associated Comment Blocks $\ldots \ldots \ldots \ldots . \ldots$

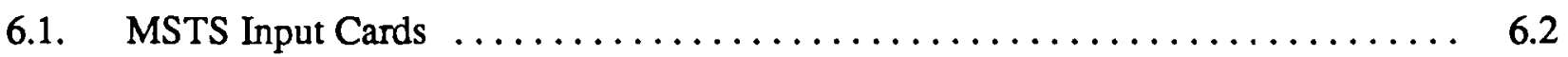

6.2. Input Reference Conventions $\ldots \ldots \ldots \ldots \ldots \ldots \ldots \ldots \ldots \ldots \ldots \ldots \ldots \ldots . . \ldots \ldots$

6.3. MSTS Field Variables Available for"Output" File, "Plot" File, and Reference Node Records ................................. 6.74

8.1. Summary of Sample Problems $\ldots \ldots \ldots \ldots \ldots \ldots \ldots \ldots \ldots \ldots \ldots \ldots \ldots \ldots$

8.2. Single-Node-Evaporation Problem Initial and Final Conditions $\ldots \ldots \ldots \ldots . \ldots$

8.3. Single-Node-Condensation Problem Initial and Final Conditions $\ldots \ldots \ldots . \ldots 8.18$

8.4. Hot Two-Phase Flow Problem Initial and Final Conditions $\ldots \ldots \ldots \ldots . \ldots . \ldots 8$

8.5. Comparison of MSTS Liquid Saturation Predictions to Philip's Semi-Analytical Solution for Vertical Infiltration of Water Into Yolo Light Clay After 2 Hours . . . . 8.29

8.6. Heat Pipe Problem Physical Properties $\ldots \ldots \ldots \ldots \ldots \ldots \ldots \ldots \ldots$ 


\section{Exhibits}

8.1. MSTS Input File for Single-Node-Evaporation Problem $\ldots \ldots \ldots \ldots \ldots . \ldots$

8.2. MSTS Output File for Single-Node-Evaporation Problem . . . . . . . . . . . 8.9

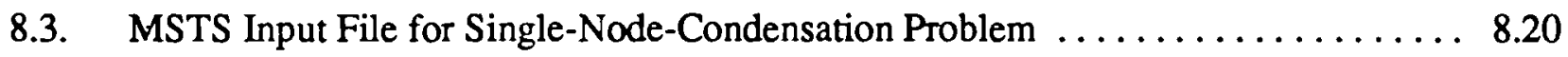

8.4. MSTS Input File for Hot Two-Phase Problem $\ldots \ldots \ldots \ldots \ldots \ldots \ldots \ldots \ldots \ldots .25$

8.5. MSTS Input File for Philip's Vertical Infiltration Problem $\ldots \ldots \ldots \ldots \ldots \ldots . \ldots$.

8.6. MSTS Input File for Two-Dimensional Infiltration Problem $\ldots \ldots \ldots \ldots \ldots . \ldots$

8.7. MSTS Input File for Two-Phase Infiltration Problem, Open Column $\ldots \ldots \ldots .46$

8.8. MSTS Input File for Two-Phase Infiltration Problem, Bounded Column . . . . . 8 8.50

8.9. MSTS Input File for Heat Pipe Problem $\ldots \ldots \ldots \ldots \ldots \ldots \ldots \ldots \ldots$

8.10. MSTS Input File for Unsaturated Transport Problem $\ldots \ldots \ldots \ldots \ldots \ldots$ 


\subsection{Introduction}

The Multiphase Subsurface Transport Simulator (MSTS) is a numerical model and computer code capable of solving fully coupled equations for conservation of water and air mass, conservation of energy, and conservation of dilute species mass in variably saturated geologic media. MSTS employs an integrated finite-difference numerical scheme in conjunction with a Newton-Raphson iteration scheme to solve the coupled equations for water conservation, air conservation, and energy conservation. The species solute mass conservation equation is solved sequentially to the coupled governing equations with a direct integrated finite-difference scheme. One-, two-, or three-dimensional domains may be simulated in a time-transient fashion.

The MSTS Graphical Input is an extremely useful pre-processor for preparing the input files that direct MSTS simulations. The interface uses a "point-and-click" approach to speed entry of geometry specification, numerical solution control, boundary and initial conditions, output directions, and geologic property values. The MSTS Graphical Input also provides elementary error checking and determines the minimum size of MSTS FORTRAN parameters required for an MSTS input file. A distinct advantage of using the MSTS Graphical Input is that this preprocessor observes all of the input file format requirements, removing the burden from the code user of learning the input file structure and constantly adhering to it.

MSTS and the MSTS Graphical Input were developed by Pacific Northwest Laboratory(a) at the request of the Yucca Mountain Project Office of the U.S. Department of Energy to produce numerical predictions of subsurface flow and transport phenomena at the potential high-level nuclear waste repository site at Yucca Mountain, Nevada.

This document is designed to introduce the user to the MSTS code, demonstrate its use, and provide a comprehensive reference of input commands used to build an MSTS input file. New users can follow the "Quick Tour" (Section 5.0) to see, step by step, how to build an input file and run a small, one-dimensional simulation. Finally, input files and results are provided for a variety of example problems to demonstrate the range of MSTS applications. Several of these example problems double as test problems, with the results compared either to analytic solutions or to other computer models. The design architecture and specific computational algorithms that compose MSTS are delineated in a companion document, the MSTS Theory Manual (White and Nichols 1992).

(a) Pacific Northwest Laboratory is operated for the U.S. Department of Energy by Battelle Memorial Institute. 


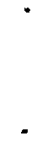




\subsection{MSTS Capabilities Overview}

The Multiphase Subsurface Transport Simulator can solve a wide variety of single- or multiplephase problems for unsaturated or saturated geologic media. The four basic governing equations it solves are conservation of water mass, conservation of air mass, conservation of thermal energy, and conservation of a dilute species mass. In this overview, the main features of the MSTS code are introduced and the fundamental governing equations are presented. Additional details on the mathematical basis and theory of the MSTS code are available in the MSTS Theory Manual (White and Nichols 1992).

\subsection{Main Features of MSTS}

By the appropriate use of the features of the MSTS computer code, a wide range of porous media problems can be solved. This section provides a brief overview of the major features of MSTS. More detailed information on the use of these features is discussed in subsequent sections of this document.

\subsubsection{Spatial Dimensionality and Coordinate Systems}

MSTS is designed to solve one-, two-, or three-dimensional problems. A problem can be defined and solved using either Cartesian or cylindrical coordinates. In a Cartesian coordinate problem, the horizontal plane is represented by $x-y$ coordinates, but for cylindrical problems the horizontal plane is represented by $r-\theta$ coordinates. The vertical coordinate, coincident with the gravity vector, is represented by $z$ in both coordinate systems.

\subsubsection{Time and Space Dependence}

All MSTS solutions are time-dependent (transient); steady-state solutions are obtained with MSTS either directly or with false transients. The values of certain parameters (e.g., sources of fluid or gas mass, thermal energy, or species mass) can be varied in time. Time-varying inputs are specified in tabular format. Time variance in parameters such as boundary conditions that are constant in time can be accommodated through use of the restart feature in MSTS.

All physical properties of a porous medium can be spatially varied by rock or soil type to model heterogeneity. There are no limits, within computer memory constraints, to the number of rock or soil types that can be defined. Certain physical properties, such as thermal or hydraulic conductivity, can be anisotropic. 


\subsubsection{Equation Coupling}

Three of the four governing equations solved by MSTS-water conservation, gas conservation, and thermal energy conservation-are solved in a fully coupled manner. The fourth equation, dilute species mass conservation, is solved sequentially to the other governing equations. Depending on the nature of the problem, any or all of the four governing equations may be activated for a particular simulation. Hence, problems that are concerned only with isothermal fluid flow would require activating only the water conservation equation. Problems involving contaminant transport in an isothermal, fully saturated environment would require solution of only the water mass conservation and species mass conservation equations.

\subsubsection{Boundary Conditions}

Boundary conditions that can be specified using MSTS include Dirichlet, Neumann, saturated, and unit-gradient conditions as applicable to each physical process. Boundaries can be defined over an entire surface, over some smaller portion of a surface, or at the interface between computational nodes and internal "inactive" (noncomputational) nodes. Boundaries are separately defined for aqueous-phase mass, gas-phase mass, vapor mass, thermal energy, and species mass.

\subsubsection{Solution Methods for Governing Equations}

Governing equations are solved by discretizing the governing equations over the spatial grid for a discrete time step and solving the resulting system of nonlinear algebraic equations. Nonlinearities in the discretized governing equations are resolved with Newton-Raphson iteration. Either a banded matrix or conjugate gradient solution scheme may be used. Where iteration is required to obtain a solution, the Newton-Raphson technique is employed.

\subsubsection{Operational Control}

Operational control is exercised by means of commands included in the input file that specify the length of simulation, time-step control, solution techniques, which of the governing equations to solve, convergence criteria for each governing equation, wallclock and CPU time limits for the simulation execution, and other aspects of simulation control. A restart capability is incorporated into MSTS to add additional flexibility.

\subsubsection{Output Control}

Output is controlled through use of commands included in the input file that permit a user to specify which field variables are recorded at which simulation steps and for which grid nodes. Output is separately controlled for the general output file, the plot file (which may be used with a 
variety of third-party graphical post-processors), and reference node output that is produced during simulation execution.

\subsubsection{Variable Saturation}

The numerical solution scheme employed in MSTS allows the solution of fluid flow in both the vadose (unsaturated) zone and the fully saturated domain. In the vadose zone, solution of the water conservation equation alone assumes that liquid water and gas (air) exist, but only liquidphase movement is modeled. If both the water and gas conservation equations are activated, then both water and gas motion is modeled.

\subsubsection{Porosity}

The MSTS code is based on a three-porosity conceptual model of porous media. These porosities are termed effective, total, and diffusive. Effective, or kinematic, porosity $\left(n_{E}\right)$ is the ratio of the volume of void space in a representative elementary volume (REV) through which water effectively advects to the total volume of the REV. Total porosity $\left(n_{T}\right)$ is the ratio of the volume of void space of a REV to the total volume of the REV. Diffusive porosity $\left(n_{D}\right)$ is the ratio of the volume of connected pore space to the total volume of the REV. Effective, diffusive, and total porosity are always subject to the physical constraint, $n_{E} \leq n_{D} \leq n_{T}$.

\subsubsection{Sources and Sinks}

Sources and sinks of thermal energy, water mass, gas mass, and dilute species mass may be included in an MSTS simulation. The strength of a source or sink either may be constant over prescribed time periods or may vary in time following a tabular specification. A source or sink is defined in relation to a node or range of nodes, giving spatial variability as required.

\subsubsection{Graphical Input}

A closely knit application that enhances the use of MSTS is the MSTS Graphical Input, a graphical user interface that makes preparation and modification of consistent, format-error-free input files radically easier than for previous hydrogeologic codes. The Graphical Input application was available for the Macintosh at the time this document was written, but versions for other computing environments may be available; check the "read.me" file on any distribution diskettes for more recent information. 


\subsubsection{Units}

MSTS performs all internal calculations using Système International (SI) units; all data and parameters are handled strictly in SI units. However, to allow for easy data entry, MSTS will read a wide variety of units from input files, including SI-derived units and English Engineering units. It accomplishes this by converting all input to SI units as it is imported. Hence, most information can be entered in the units in which it was originally recorded, while the advantages of working with SI units are retained.

\subsection{Application Scope}

The four fundamental equations for conservation of component mass, energy, and species mass that compose MSTS essentially define the simulator's application scope. A fundamental restriction with respect to MSTS's application scope is that all applications are strictly limited to subsurface porous media environments. This restriction holds because of the application of Darcy's law to compute phase volumetric fluxes. Furthermore, driving forces for fluid flow are limited to pressure gradients and gravitational body forces. MSTS is classified as a twocomponent, two-phase simulator. This classification indicates that the simulator is capable of computing flow and transport processes for subsurface systems that contain no more than two components and two phases. Moreover, components are confined to water and air, and phases are confined to aqueous and gas. Subsurface systems contaminated with significant concentrations of an organic compound fall outside of MSTS's application scope. Low solubilities are assumed in expressing the quantity of air dissolved in the aqueous phase. Within the gas phase, component mass fractions are completely variable. Liquid saturations and relative permeabilities are computed neglecting hysteretic and air entrapment effects. The flow model for highly fractured systems assumes that the porous medium and the fractures are continuously in thermal and hydraulic equilibrium. Although discrete fractures can be modeled with MSTS, the simulator is inappropriate for modeling transient fracture-matrix interactions with the dual-porosity model.

The energy conservation equation expands the application scope by allowing thermal gradients to influence the flow fields. The energy equation formulation allows for both sensible and latent heat transfer. Sensible heat transfer occurs by thermal diffusion through the solid and aqueous phases and by advection through the aqueous phase. Diffusive heat transfer through the gas phase is neglected. Latent heat transfer by evaporation and condensation processes is considered for the water component. Because interphase mass transfer computations depend on the assumption of thermodynamic equilibrium, MSTS is not applicable for systems requiring disequilibrium or kinetic 
modeling of the thermodynamic processes. Sensible and latent heat transfer associated with diffusing or dispersing components is neglected. Therefore, enhanced heat transfer by vapor migration under thermal gradient conditions must be included empirically into the effective thermal conductivity coefficient.

Species transport processes are solved by assuming loose coupling between multiphase fluid flow and species transport processes. Because of the strong dependence of phase pressures and temperature on the secondary variables that compose the fundamental equations for mass and energy conservation, these fluid flow and heat transport fundamental equations are solved simultaneously (coupled). The principal assumption associated with the species transport processes is that the species are numerically considered passive scalars. Physically, this assumption implies that the secondary variables of the mass and energy conservation equations are independent of species concentrations. A passive scalar species limits the applicability of the engineering simulator to dilute species concentrations. Species concentrations may be considered dilute whenever the physical, thermal, or transport properties of the transport fluid remain unaltered. The dilute concentration assumption and decoupled solution approach also allow consideration of multiple species and transport modeling of radioactive decay products. No accounting is made of chemical reaction and/or radioactive decay products.

\subsection{Governing Equations}

MSTS employs four partial differential equations as the governing equations to mathematically describe the transport of water, air, thermal energy, and a dilute species in the subsurface environment. Each partial differential equation represents an expression of conservation for either component mass, thermal energy, or the species mass. The conservation equations balance the time rate of change in the conserved quantity within a control volume against the surface fluxes of the conserved quantity entering or leaving the control volume. The conserved quantities are water mass, air mass, thermal energy, and species mass. Transport of the four conserved quantities through a porous medium is modeled within two distinct phases: aqueous and gas. The aqueous phase is composed primarily of water, with relatively small amounts of dissolved air, whereas the gas phase is composed of various compositions of air and water vapor.

\subsubsection{Water Mass Conservation Equation}

The conservation equation for water mass, expressed in partial differential equation form, is shown in Equation (2.1). The term on the left-hand side of Equation (2.1) represents the time rate of change of water mass within the control volume. Water occurs within the soil pores as liquid in 
the aqueous phase and as vapor in the gas phase. Unconnected pore spaces are considered to be filled with liquid water but isolated from the continuous pore spaces, and therefore do not contribute to the water mass accumulation term. Interphase mass transfer of water between the aqueous and gas phases is computed by assuming thermodynamic equilibrium conditions. This equilibrium assumption implies that time scales for thermodynamic processes are significantly shorter than for those associated with transport. The two gradient terms on the right-hand side of Equation (2.1) represent the net mass flux of water mass into the control volume through the aqueous and gas phases, respectively. Water mass flux occurs by advection in both phases and by diffusion through the gas phase. Advective transport is governed by Darcy's law for flow through porous media, where phase pressure gradients and gravitational forces drive the flow fields. For unsaturated porous media, advective transport depends on saturation-dependent relative permeabilities for each phase. Component diffusion rates through the aqueous phase are neglected as a transport mechanism. Diffusion through the gas phase is governed by the classical mass diffusion equation, modified by the porous media tortuosity. The driving forces behind diffusive transport are component concentration or mass fraction gradients in the gas phase. The last term on the righthand side of Equation (2.1) represents the water mass source rate within the control volume.

$$
\begin{aligned}
& \frac{\partial}{\partial t}\left[n_{D} x_{\ell}^{w} \rho_{\ell} s_{\ell}+n_{D} x_{g}^{\omega} \rho_{g} s_{g}\right]=\nabla\left[\frac{x_{\ell}^{\omega} \tilde{k} k_{r \ell} \rho_{\ell}}{\mu_{\ell}}\left(\nabla P_{\ell}+\rho_{\ell} g z\right)\right] \\
& +\nabla\left[\frac{x_{g}^{w} \tilde{k} k_{r g} \rho_{g}}{\mu_{g}}\left(\nabla P_{g}+\rho_{g} g \hat{z}\right)+\tau_{g} n_{D} \rho_{g} s_{g} D_{g}^{a w} \nabla x_{g}^{w}\right]+\dot{m}^{w}
\end{aligned}
$$

\subsubsection{Air Mass Conservation Equation}

The conservation equation for air mass, expressed in partial differential equation form, is shown in Equation (2.2). The term on the left-hand side of Equation (2.2) represents the time rate of change of air mass in the control volume. Air occurs within the soil pores as dissolved air in the aqueous phase and as gaseous air the gas phase. Occluded air within the aqueous phase is neglected, by assuming that air entrapped by imbibing water would eventually dissolve into the aqueous phase. Unconnected pore spaces are considered to be filled with liquid water, but isolated 
from the continuous pore spaces, and therefore do not contribute to the air mass accumulation term. Interphase mass transfer of air between the aqueous and gas phases is computed by assuming thermodynamic equilibrium conditions. This equilibrium assumption implies that time scales for thermodynamic processes are significantly shorter than for those associated with transport. The two gradient terms on the right-hand side of Equation (2.2) represent the mass flux of air mass into the control volume through the aqueous and gas phases, respectively. Air mass flux occurs by advection in both phases and by diffusion through the gas phase. Advective transport is governed by Darcy's law for flow through porous media, where phase pressure gradients and gravitational forces drive the flow fields. For unsaturated porous media, advective transport depends on saturation-dependent relative permeabilities for each phase. Component diffusion rates through the aqueous phase are neglected as a transport mechanism. Diffusion through the gas phase is governed by the classical mass diffusion equation, modified by the porous media tortuosity. The driving forces behind diffusive transport are component concentration or mass fraction gradients in the gas phase. The last term on the right-hand side of Equation (2.2) represents the air mass source rate within the control volume.

$$
\begin{aligned}
& \frac{\partial}{\partial t}\left[n_{D} x_{\ell}^{a} \rho_{\ell} s_{\ell}+n_{D} x_{g}^{a} \rho_{g} s_{g}\right]=\nabla\left[\frac{x_{\ell}^{a} \tilde{k} k_{r \ell} \rho_{\ell}}{\mu_{\ell}}\left(\nabla P_{\ell}+\rho_{\ell} g z\right)\right. \\
& +\nabla\left[\frac{x_{g}^{a} \tilde{k} k_{r g} \rho_{g}}{\mu_{g}}\left(\nabla P_{g}+\rho_{g} g \hat{z}\right)-\tau_{g} n_{D} \rho_{g} s_{g} D_{g}^{a w} \nabla x_{g}^{w}\right]+\dot{m}^{a}
\end{aligned}
$$

\subsubsection{Thermal Energy Conservation Equation}

The conservation equation for thermal energy, expressed in partial differential form, is shown in Equation (2.3), where phase velocities are defined by Equations (2.4) and (2.5) for the aqueous and gas phases, respectively. The term on the left-hand side of Equation (2.3) represents the time rate of change of heat content in the control volume. The total heat contained in the control volume depends directly on temperature, liquid saturations, phase compositions, and soil physical properties. For the thermal energy conservation equation, a modified aqueous-phase saturation applies that accounts for the thermal capacitance of unconnected pore spaces, which are assumed to 
be filled with liquid water. Internal energies and densities of the aqueous phase are those for pure liquid water. This assumption follows directly from the principal assumption of relatively low solubilities of air in the aqueous phase. Disparately, the internal energy of the gas phase is strongly dependent on phase composition. The first term on the right-hand side of Equation (2.3) represents heat transfer into the control volume by advection. Analogous to the evaluation of internal energies, the enthalpy of the aqueous phase is that for pure liquid water, whereas the gas-phase enthalpy is strongly dependent on phase composition. The second term on the right-hand side of Equation (2.3) represents heat transfer by conduction and mechanical dispersion. The equivalent conductivity tensor represents the combination of solid-and liquid-phase conductivities along with the mechanical dispersion coefficient. Typically, the mechanical dispersion tensor is neglected by assigning zero to the longitudinal and transverse dispersivities. Thermal conductivity through the gas phase is neglected. The last three terms on the right-hand side of Equation (2.3) represent internal heat generation quantities from thermal energy sources and sinks within the control volume, and the change in internal energy associated with water and air mass sources and sinks within the control volume, respectively. The internal heat generation from mass sources represent the enthalpy associated with a mass source.

$$
\begin{gathered}
\frac{\partial}{\partial t}\left[\left(1-n_{T}\right) \rho_{s} u_{s}+\eta_{D} \rho_{\ell} u_{\ell}+n_{D} s_{g} \rho_{g} u_{g}\right]= \\
-\nabla\left[\rho_{\ell} h_{\ell} \mathbf{V}_{\ell}+\rho_{g} h_{g} \mathbf{V}_{g}\right]+\nabla\left[\tilde{k}_{e} \nabla T\right]+\dot{q}+h^{w} m^{\cdot w}+h^{a} \dot{m}^{a}
\end{gathered}
$$

where

$$
\begin{aligned}
& \mathrm{V}_{\ell}=A \frac{\tilde{k} k_{r \ell}}{\mu_{\ell}}\left(\nabla P_{\ell}+\rho_{\ell} g \hat{z}\right) \\
& \mathrm{V}_{g}=A \frac{\tilde{k} k_{r g}}{\mu_{g}}\left(\nabla P_{g}+\rho_{g} g z\right)
\end{aligned}
$$




\subsubsection{Species Mass Conservation Equation}

The conservation equation for species mass, expressed in partial differential equation form, is shown in Equation (2.6). The term on the left-hand side of Equation (2.6) represents the time rate of change of total species mass within a control volume. Interphase species mass transfer between solid and fluid phases is computed by assuming that thermodynamic and geochemical equilibrium conditions apply. This assumption of equilibrium conditions requires that time scales for thermodynamic and geochemical phenomena be small when compared with those for transport phenomena. For transport through geologic media, these assumptions are generally appropriate. The first term on the right-hand side of Equation (2.6) represents the migration of species mass into the control volume by advection through the aqueous and gas phases. Advective transport is dependent on the species concentration and Darcy velocity within each phase. The second term on the right-hand side of Equation (2.6) represents the hydrodynamic dispersion of species in the aqueous phase. The third term on the right-hand side of Equation (2.6) represents the diffusion of species mass into the control volume in the aqueous and gas phases. Typically, diffusion coefficients for the gas phase will be several orders of magnitude greater than those for the aqueous phase. Species diffusion fluxes are phase specific and depend on the gradients in species phase concentrations, phase pore areas, and phase tortuosities. The fourth term on the right-hand side of Equation (2.6) represents the generation of species within the control volume. The last term on the right-hand side of Equation (2.6) represents the radioactive decay or consumption rate of species mass.

$$
\begin{gathered}
\frac{\partial C}{\partial t}=-\nabla\left[C_{\ell} \mathbf{v}_{\ell}+C_{g} \mathbf{v}_{g}\right]+\nabla\left[s_{\ell} n_{D} \tilde{D}_{h_{\ell}} \nabla C_{\ell}\right] \\
+\nabla\left[\tau_{\ell} s_{\ell} n_{D} D_{d_{\ell}} \nabla C_{\ell}+\tau_{g} s_{g} n_{D} D_{d_{g}} \nabla C_{g}\right]+\dot{s}_{c}-\dot{R}_{c} C
\end{gathered}
$$

\subsection{Solution Options}

One of the design goals for MSTS was to create an efficient numerical simulator for predicting flow and transport phenomena in the subsurface environment. Because of the diversity of subsurface flow problems, a single-solution option would result in a numerical simulator that was computationally inefficient for a majority of problems. For example, if the simulation problem involved generating predictions of radionuclide transport by ground water under isothermal 
conditions, then it would be superfluous to include gas-phase transport and heat transfer in the solution scheme. The preferred solution scheme in terms of computational efficiency would be to sequentially solve the water mass conservation and species mass conservation equations. However, if the simulation problem involves nonisothermal aspects and if species transport through the gas phase is significant, then the solution scheme would require the coupled solution of both component mass conservation equations and the energy equation, along with the sequential solution of the species transport equation. To avoid simulating the negligible aspects of a particular subsurface flow and transport problem, several solution options are available with MSTS. This approach yields a simulator that depends on the problem characteristics.

Solution modes or options are characterized by the specific solved conservation equations. The conservation equations are divided into coupled and auxiliary conservation equations according to their interdependencies. The governing conservation equations for water mass, air mass, and energy are considered coupled equations because they are solved simultaneously. The species mass conservation equation is considered an auxiliary equation because it is solved sequentially to the coupled conservation equations. The lowest-order solution modes are those that involve the solution of a single coupled conservation equation (e.g., water mass conservation, air mass conservation, or energy conservation). These lower-order solution modes generally require more assumptions about the subsurface system to be applicable. The next-higher-order solution modes are those that involve the coupled solution of a pair of conservation equations. These solution modes typically eliminate one of the primary assumptions associated with a single-equation solution mode. The highest-order solution mode requires the coupled solution of both component mass conservation equations and the energy conservation equation. The computational effort increases roughly with the square of the coupled equations solved (i.e., three coupled equations require eight times the computational effort required for a single equation). All solution modes can include the solution of the auxiliary species conservation equation.

Solution modes are chosen by the user according to the coupled conservation equations selected for solution. Considerable computational savings may be achieved through the proper choice of a solution mode for a particular application. Choosing lower-order solution modes (i.e., those with fewer equations) generally requires that some assumptions be made about the problem. Some assumptions are obvious (e.g., eliminating the air mass conservation equation for isothermal ground-water flow problems, or eliminating the energy conservation equation for isothermal problems). Other assumptions require previous modeling experience, experimental evidence, or field observations to support the elimination of a specific equation. For example, what thermal gradients constitute the inclusion of vapor transport through the gas phase to accurately predict heat 
transfer rates? Answering this type of question requires specific property information about the porous media and anticipated thermal gradients.

The following sections (2.4.1 through 2.4.7) describe the seven solution modes available in MSTS. Each section is labeled with a solution mode title, followed by brief general description of the solution mode and the type of problems to which it applies. Each section also contains primary, secondary, and velocity variable lists. Primary variables refer to the principal unknowns for the solution mode. The number of primary variables and the number of solved equations always coincide. Fixed primary variables refer to primary variables that must be specified according to the problem assumptions. Fixed primary variables are specified, not computed. For example, the gasphase pressure for a ground-water flow problem solved in the Water mode might be fixed to atmospheric conditions, or 101,325.0 Pa. Secondary variables are physical properties, transport properties, and saturations that are computed from the unknown and fixed primary variables. Fixed secondary variables are physical properties, transport properties, and saturations, required for the solved equations, that are independent of the unknown primary variables. For example, liquid viscosities generally are considered independent of liquid pressures. Velocities refer to flow velocities or diffusion mass fluxes computed by the engineering simulator from the solution of the unknown pressure field. Fixed velocities are those velocities or diffusion mass fluxes computed for inactive phases from fixed pressure fields, soil characteristics, and transport properties. 


\subsubsection{Water}

Description: The Water mode solves saturated and unsaturated ground-water flow problems without a participating gas phase. Soil characteristics are computed for a two-phase air-water system using nonhysteretic relations. Physical properties may be specified invariant or dependent on aqueous pressure. Species transport in the aqueous phase may be modeled by additionally solving the species mass conservation equation.

Application: Appropriate applications for the Water mode include saturated and unsaturated ground-water flow problems with negligible thermal and gas pressure gradients.

Specification: Solution Schemes card

Governing Equations

Thermal Energy Conservation Equation: off

Water Conservation Equation: on

Air Conservation Equation: off

Species Conservation Equation: off / on

Options

Binary Diffusion: off

Fixed Properties: off / on

Liquid Phase: on

Gas Phase: off

Vapor Pressure Lowering: off

Primary Variables: aqueous pressure

Fixed Primary Variables: gas pressure, temperature

Secondary Variables: aqueous-phase density, aqueous-phase saturation, aqueous-phase relative permeability, porosity, aqueous-phase tortuosity

Fixed Secondary Variables: aqueous-phase viscosity

Eluxes: aqueous-phase velocity 


\subsubsection{Air}

Description: The Air mode solves unsaturated subsurface gas flow problems with static saturation fields. Liquid saturations are temporally invariant, and are either specified or computed from initial fields of aqueous pressure and gas pressure. Fixed physical properties are temporally invariant, computed from the initial fields of aqueous pressure, gas pressure, and temperature. Vapor diffusive transport and convective mass transport may be computed through the gas phase for spatially variant temperature fields. Conservation of water mass and energy is not ensured for vapor transport problems. Gas-phase density, gas-phase mass fractions, and vapor diffusivities may be specified invariant or dependent on gas pressure. Species transport in the gas phase can be modeled by additionally solving the species mass conservation equation.

Application: Appropriate applications for the Air mode include unsaturated subsurface gas flow problems with invariant liquid saturation and temperature fields. Density-driven gas flows resulting from steady thermal gradients would be an appropriate problem for this mode. For these types of applications, the influence of both temperature and vapor mass fractions on gas-phase properties could be investigated. An inappropriate application, however, would result if either the liquid saturation or the thermal fields were significantly altered by the transport of vapor or heat through the gas phase.

Specification: Solution Schemes card

Governing Equations

Thermal Energy Conservation Equation: off

Water Conservation Equation: off

Air Conservation Equation: on

Options

Species Conservation Equation: off / on

Binary Diffusion: off / on

Fixed Properties: off / on

Liquid Phase: off

Gas Phase: on

Vapor Pressure Lowering: off / on

Primary Variables: gas pressure

Fixed Primary Variables: aqueous pressure, temperature

Secondary Variables: water-vapor partial pressure, air partial pressure, water-vapor density, air density, gas-phase density, water-vapor mass/mole fraction, air mass/mole fraction, gas-phase viscosity, water-vapor diffusivity

Fixed Secondary Variables: aqueous-phase saturation

Fluxes: gas-phase velocity, water-vapor diffusion mass flux 


\subsubsection{Water-Air}

Description: The Water-Air mode solves saturated and unsaturated ground-water flow problems with a participating gas phase. Soil characteristics are computed for a two-phase airwater system using nonhysteretic relations. Physical properties are temporally invariant, computed from the initial fields of aqueous pressure, gas pressure, and temperature. Watervapor transport through the gas phase is computed for spatially variant temperature fields. Phase densities, mass fractions, and vapor diffusivities may be specified invariant or dependent on pressure. Species transport in the aqueous and gas phases can be modeled by additionally solving the species mass conservation equation.

Application: Appropriate applications for the Water-Air mode include saturated and unsaturated ground-water flow problems with steady or negligible thermal gradients. This mode differs from the Water mode because it is applicable to problems involving gas pressure gradients. For steady thermal conditions, this mode is appropriate for predicting the transport of water vapor by diffusion and advection through the gas phase. The mode becomes inappropriate when the liquid and mass transport influences the thermal field. Because heat transfer associated with vaporization and condensation may be significant, thermal effects should be considered carefully before applying this mode to a simulation problem.

Specification: Solution Schemes card

Governing Equations

Thermal Energy Conservation Equation: off

Water Conservation Equation: on

Air Conservation Equation: on

Options

Species Conservation Equation: off / on

Binary Diffusion: off / on

Fixed Properties: off / on

Liquid Phase: on

Gas Phase: on

Vapor Pressure Lowering: off / on

Primary Variables: aqueous pressure, gas pressure

Fixed Primary Variables: temperature

Secondary Variables: water-vapor partial pressure, air partial pressure, water-vapor density, air density, gas-phase density, aqueous-phase density, air mass/mole fraction, water-vapor mass/mole fraction, aqueous-phase air mass/mole fraction, gas-phase viscosity, aqueous-phase saturation, gas-phase saturation, aqueous-phase relative permeability, gas-phase relative permeability, porosity, aqueous-phase tortuosity, gas-phase tortuosity, water-vapor diffusivity

Fixed Secondary Variables: aqueous-phase viscosity

Fluxes: aqueous-phase velocity, gas-phase velocity, water-vapor diffusion mass flux 


\subsubsection{Energy}

Description: The Energy mode solves heat transfer problems for saturated and unsaturated subsurface flow problems. Constant aqueous- and gas-phase flow fields may be prescribed. Diffusive heat transfer occurs through the solid and liquid phases. Advective heat transfer occurs through the fluid phases. No heat transfer occurs by vapor diffusion through the gas phase. System pressures, phase saturations, and relative permeabilities are invariant. Mass continuity is not ensured for specified flow fields. Physical properties may be specified as invariant or temperature dependent.

Application: Appropriate applications for the Energy mode include subsurface heat transfer through saturated and unsaturated porous media. Fixed saturation and fluid flow fields are the principal assumptions associated with this mode. Saturation levels and Darcy flow velocities may be specified for the aqueous and gas phases through the initial pressure fields and soil characteristics. An appropriate application for this mode could involve heat transfer through completely dry or completely saturated porous media, or heat transfer through partially saturated porous media with relatively high liquid mass flux rates. Without adjusting the porous media thermal conductance for heat transfer enhancement by vapor diffusion, this mode would be inappropriate for predicting heat transfer for partially saturated media with low liquid mass flux rates. The mode would also be inappropriate for conditions where the thermal field affected the saturation fields (e.g., heat-pipe flow phenomena).

Specification: Solution Schemes card

Governing Equations

Thermal Energy Conservation Equation: on

Water Conservation Equation: off

Air Conservation Equation: off

Options

Species Conservation Equation: off

Binary Diffusion: off

Fixed Properties: off / on

Liquid Phase: off / on

Gas Phase: off / on

Vapor Pressure Lowering: off

Primary Variables: temperature

Fixed Primary Variables: aqueous pressure, gas pressure

Secondary Variables: water-vapor partial pressure, air partial pressure, water-vapor density, air density, gas-phase density, aqueous-phase density, water-vapor mass/mole fraction, air mass/mole fraction, aqueous-phase air mass fraction, water-vapor enthalpy, air enthalpy, gasphase enthalpy, aqueous-phase enthalpy, water-vapor internal energy, air internal energy, gasphase internal energy, aqueous-phase internal energy, aqueous-phase thermal conductivity, solid-phase thermal conductivity, equivalent thermal conductivity

Fixed Secondary Variables: aqueous-phase saturation

Fluxes: thermal flux 


\subsubsection{Water-Energy}

Description: The Water-Energy mode solves aqueous flow and heat transfer problems for saturated and unsaturated subsurface flow systems. Aqueous-phase flow fields are computed assuming a nonparticipating gas phase. Soil characteristics are computed for an air-water system using nonhysteretic relations. Diffusive heat transfer occurs through the solid and aqueous phases. Advective heat transfer occurs through the aqueous phase. No heat transfer occurs by vapor diffusion through the gas phase. Physical properties may be specified invariant or dependent on aqueous pressure and temperature. Species transport in the aqueous phase may be modeled by additionally solving the species mass conservation equation.

Application: Appropriate applications for the Water-Energy mode include ground-water flow and heat transfer through saturated and unsaturated porous media. Typical applications would involve concurrent solutions of the aqueous pressure and temperature fields. Transport of heat and water vapor through the gas phase is assumed negligible with this mode. An appropriate application for this mode could be the solution of a density-driven aqueous flow through porous media. Inappropriate problems include those characterized with high latent heat transfer rates through the gas phase.

Specification: Solution Schemes card

Governing Equations

Thermal Energy Conservation Equation: on

Water Conservation Equation: on

Air Conservation Equation: off

Options

Species Conservation Equation: off / on

Binary Diffusion: off

Fixed Properties: off / on

Liquid Phase: on

Gas Phase: off / on

Vapor Pressure Lowering: off

Primary Variables: aqueous pressure, temperature

Fixed Primary Variables: gas pressure

Secondary Variables: aqueous-phase density, aqueous-phase viscosity, aqueous-phase enthalpy, aqueous-phase internal energy, aqueous-phase thermal conductivity, solid-phase thermal conductivity, equivalent thermal conductivity, aqueous-phase saturation, trapped gas saturation, aqueous-phase relative permeability, porosity, aqueous-phase tortuosity

Fluxes: aqueous-phase velocity, thermal flux 


\subsubsection{Air-Energy}

Description: The Air-Energy mode solves unsaturated subsurface gas flow and heat transfer problems with static saturation fields. Liquid saturations are temporally invariant, and are either specified or computed from initial fields of aqueous pressure and gas pressure. Diffusive heat transfer occurs through the solid and liquid phases. Advective heat transfer occurs through the gas phase. No heat transfer occurs by vapor diffusion through the gas phase. Conservation of water mass is not ensured for vapor transport problems. Physical properties may be specified invariant or dependent on gas pressure and temperature. Species transport in the gas phase may be modeled by additionally solving the species mass conservation equation.

Application: Appropriate applications for the Air-Energy mode include gas flow and heat transfer through unsaturated porous media. Density-driven gas flows resulting from transient or steady thermal fields would be an appropriate problem for this mode. Fixed liquid saturation fields may be specified for the aqueous phase through the initial aqueous and gas pressure fields and the soil characteristics. For these types of applications, the influence of both temperature and vapor mass fractions on gas-phase properties could be investigated. An inappropriate application, however, would result if the liquid saturation fields were significantly altered by the transport of vapor or heat through the gas phase.

Specification: Solution Schemes card

Governing Equations

Thermal Energy Conservation Equation: on

Water Conservation Equation: off

Air Conservation Equation: on

Options

Species Conservation Equation: off / on

Binary Diffusion: off / on

Fixed Properties: off / on

Liquid Phase: off

Gas Phase: on

Vapor Pressure Lowering: off / on

Primary Variables: gas pressure, temperature

Fixed Primary Variables: aqueous pressure

Secondary Variables: water-vapor partial pressure, air partial pressure, water-vapor density, air density, gas-phase density, water-vapor mass/mole fraction, air mass/mole fraction, gas-phase viscosity, water-vapor enthalpy, air enthalpy, gas-phase enthalpy, aqueous-phase enthalpy, water-vapor internal energy, air internal energy, gas-phase internal energy, aqueous-phase internal energy, aqueous-phase thermal conductivity, solid-phase thermal conductivity, equivalent thermal conductivity, water-vapor diffusivity

Fixed Secondary Variables: aqueous-phase saturation

Fluxes: gas-phase velocity, water-vapor diffusion mass flux, thermal flux 


\subsubsection{Water-Air-Energy}

Description: The Water-Air-Energy mode solves aqueous flow and heat transfer problems for saturated and unsaturated subsurface flow systems. Aqueous-phase flow fields are computed assuming a participating gas phase. Soil characteristics are computed for an air-water system using nonhysteretic relations. Diffusive heat transfer occurs through the solid and aqueous phases. Advective heat transfer occurs through the aqueous and gas phases. Diffusive heat transfer through the gas phase includes both sensible and latent components for water vapor. No heat transfer occurs by vapor diffusion through the gas phase. Physical properties may be specified invariant or dependent on aqueous pressure, gas pressure, and temperature. Species transport in the aqueous and gas phases can be modeled by additionally solving the species mass conservation equation.

Application: Appropriate applications for the Water-Air-Energy mode include aqueous and gas flow and heat transfer through saturated and unsaturated porous media. Because of the coupled solution of the water, air, and energy equations, this mode is applicable to problems involving both sensible and latent heat transfer through the gas phase (e.g., heat-pipe flow). This mode eliminates the isothermal assumptions associated with the Water-Air mode and the assumptions of zero latent heat transfer associated with the Water-Energy mode, at a cost of additional computational effort.

Specification: Solution Schemes card

Governing Equations

Thermal Energy Conservation Equation: on

Water Conservation Equation: on

Air Conservation Equation: on

Species Conservation Equation: off / on

Options

Binary Diffusion: off / on

Fixed Properties: off / on

Liquid Phase: on

Gas Phase: on

Vapor Pressure Lowering: off / on

Primary Variables: aqueous pressure (water-vapor mass fraction), gas pressure, temperature

Secondary Variables: water-vapor partial pressure, air partial pressure, water-vapor density, air density, gas-phase density, aqueous-phase density, air mass/mole fraction, water-vapor mass/mole fraction, aqueous-phase air mass/mole fraction, gas-phase viscosity, aqueous-phase viscosity, water-vapor enthalpy, air enthalpy, gas-phase enthalpy, aqueous-phase enthalpy, water-vapor internal energy, air internal energy, gas-phase intemal energy, aqueous-phase internal energy, aqueous-phase thermal conductivity, solid-phase thermal conductivity, equivalent thermal conductivity, aqueous-phase saturation, gas-phase saturation, trapped gas saturation, aqueous-phase relative permeability, gas-phase relative permeability, porosity, aqueous-phase tortuosity, gas-phase tortuosity, water-vapor diffusivity

Fluxes: aqueous-phase velocity, gas-phase velocity, water-vapor diffusion mass flux, thermal flux 


\subsection{Graphical Input Overview}

The MSTS Graphical Input is an independent application that provides a relatively easy way to prepare MSTS input files. The traditional way to prepare simulation input/control files for numerical simulators is to use a text editor and type in the necessary information following the format required by the particular simulation code to be used. This is still possible with the MSTS code, but the MSTS Graphical Input provides an alternative point-and-click method that simplifies input file preparation.

The MSTS Graphical Input has three functions: 1) to prepare MSTS input files, 2) to perform a few elementary consistency checks on these files, and 3) to compute the minimum size of certain MSTS code parameters for a particular input file. The input file written by MSTS Graphical Input is an ASCII text file that exactly meets the input file format requirements of the MSTS code. This format is given in detail in Section 6.0 (Input File Structure).

The MSTS Graphical Input includes another feature to enhance its usefulness; online, contextsensitive help is available at any time the Graphical Input is in use. To activate this feature, press [98) (command and $\mathrm{H}$ keys together) or select Help from the Window menu. The help window that appears will contain information on the input card that is currently open. Information on other subjects is available by selecting topics from the main Help menu or by searching on keywords. Close the help window when finished to return to the input card last active.

For a complete introduction to the use of the MSTS Graphical Input, refer to Section 5.0 (MSTS Quick Tour). The Quick Tour will guide you through a small, but complete, numerical simulation of vertical water infiltration to demonstrate the use of the MSTS Graphical Input and the MSTS computer code. 



\subsection{File Management}

MSTS input/output and source code file management is relatively straightforward and simple. This section is not highly specific about file management because MSTS can be used on such a variety of platforms (e.g., Macintosh, Cray, Sun, Convex, and HP). Instead, a few general guidelines common to the use of MSTS on any computing platform are provided. It is the responsibility of the users to adapt these guidelines to their particular computer platforms and operating systems.

\subsection{MSTS Input and Output Files}

All input and output files used by the MSTS code are listed in Table 4.1 with a brief description of the function or contents of each. All input and simulation control is accomplished using the "input" file, whereas output is directed to several of the files named in Table 4.1. This "input" file may be prepared either with a text editor or by using the MSTS Graphical Input. The Graphical Input is described in Section 3.0, and a detailed reference of the input file format is provided in Section 6.0. The MSTS code always searches for the file names listed in Table 4.1 in the default directory during execution.

If an output file already exists in the default directory with the same file name as one MSTS must use, it will overwrite the existing file. Therefore, to keep copies of output files from previous simulations, the user must either 1) maintain all input and output files associated with a single simulation in a separate directory or 2) rename the input and output files that are to be kept with file names that do not appear in Table 4.1. In the first approach, the user typically would give the directory a name descriptive of the simulation (e.g., "sim1" or "run03"). In the second approach, the user should give file names that group the simulation together, such as renaming the "output," "surface," and "debug" files to "run01.out," "run01.srf," and "run01.dbg," respectively.

The restart file requires special attention. If a simulation requires a restart file, the file must be in the default directory and must be named "restart". Be cautious, however, MSTS will read the existing restart file, perform the simulation, and then overwrite the restart file with the conditions at the end of the new simulation. Hence, to keep a copy of the original restart file, which is recommended in case the restarted simulation must be repeated for any reason, the user should make a copy of the restart file prior to using it. If using a separate directory for each simulation, simply copy the restart file to the directory intended for the new simulation. If renaming files by 
Table 4.1. MSTS Input and Output File Name Convention

\begin{tabular}{|c|c|c|}
\hline File Name & Type(a) & Contents \\
\hline input & $\mathrm{i}$ & parameter input and simulation control commands \\
\hline output & 0 & $\begin{array}{l}\text { echo of simulation input, simulation progress, and selected } \\
\text { field variable output }\end{array}$ \\
\hline restart & io & values of field variables in model domain at end of simulation \\
\hline surface & 0 & surface flux integrator output \\
\hline debug & o & optional debugging information \\
\hline
\end{tabular}

(a) $\mathrm{i}=$ input file (read by MSTS)

$0=$ output file (written by MSTS)

io = both input and output (written by MSTS for later use)

simulation, copy the restart file (rather than renaming it) to some non-MSTS file name (e.g., "run01.res").

\subsection{MSTS Source Code Parameterization}

The MSTS source code file, "msts.f," is a text file that contains approximately 20,000 lines of source code and comments written in FORTRAN 77, following the American National Standards Institute (ANSI) standards. A user must usually make two general modifications to the source code before executing the code: parameterization and machine specification (discussed in Section 4.3). Parameterization involves modifying the parameter statements in the source code to fit a particular class of problems. This section presumes that users are familiar with the operating systems and text editors for their computing environments.

To parameterize a code, first determine the size of each parameter. Table 4.2 provides a list of MSTS parameters and their functions and computations. The computations provided in Table 4.2 give the minimum size of each parameter, these can be larger, but larger parameters will increase the size of the executable file. It is usually best to compute the largest value of each parameter for all simulations of a particular problem you wish to run and parameterize the MSTS code with those values. Once you have determined the parameter values, use a text editor to open the MSTS source code file and navigate to the first parameter statement block (usually within the first 50 lines of source code); it will look like this: 
Table 4.2. MSTS Parameters

Parameter

LFX

LFY

LFZ

LFLD

LROW

LCOUP

$\mathrm{LZN}$

LSN

LBC

LST

LSCT

LRPT

LSF

LCHR maximum number of $x$ - or $r$-nodes

maximum number of $y$ - or $\theta$-nodes

maximum number of $z$-nodes

maximum number of nodes (LFLD $=\mathrm{LFX} * \mathrm{LFY} * \mathrm{LFZ})$

Minimum value of LROW depends on the solution scheme chosen. For any method, ISLVS is the number of coupled equations that will be solved and ranges from 1 to 3 (add 1 each for thermal energy, water mass, and air mass conservation equations; species mass is not a coupled equation and does not affect ISLVS).

Banded Matrix: $\quad$ Compute IJFLD $=$ LFX * LFY

Compute JKFLD $=$ LFY * LFZ

Compute KIFLD $=$ LFX $*$ LFZ

If IJFLD $\leq$ JKFLD and IJFLD $\leq$ KIFLD, then

LROW $=3 *($ ISLVS * IJFLD + ISLVS -1$)+2$

Else if JKFLD $\leq$ KIFLD and JKFLD $\leq$ IJFLD then

LROW $=3 *$ (ISLVS * JKFLD + ISLVS - 1) + 2

Else if KIFLD $\leq$ IJFLD and KIFLD $\leq$ JKFLD then

LROW $=3 *($ ISLVS * KIFLD + ISLVS - 1) +2

Conjugate Gradient: ROW = ISLVS * LFLD + 1

(LFLD - NPX) * ISLVS + 1, where NPX is the total number of inactive nodes

maximum number of rock or soil type declarations

maximum number of source term declarations

maximum number of boundary node conditions declared for any one boundary condition card (maximum number of nodes with specified boundary conditions in liquid boundary, gas boundary, vapor boundary, thermal energy boundary, or species boundary condition cards).

maximum number of entries in all source term tables together

maximum number of rock or soil characteristic declarations

maximum number of relative-permeability declarations

maximum number of surface flux integrator declarations

maximum number of characters in any input card (usually set to 1000 for convenience) 


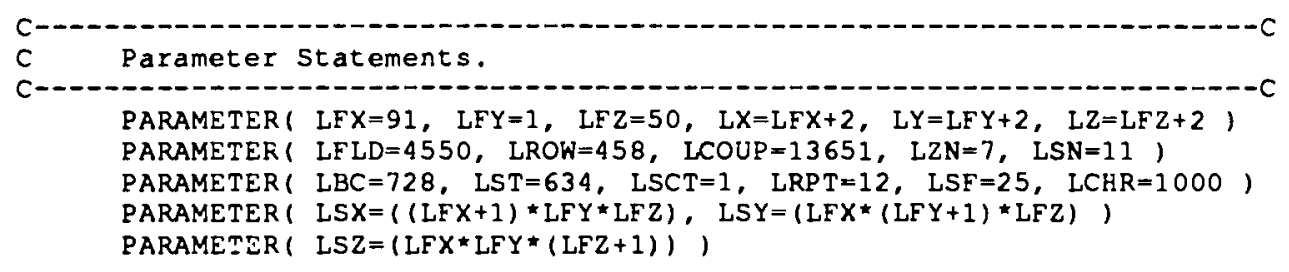

Only the first three statements are ever changed; the last two (LSX, LSY and LSZ) never require modification. These parameter statements appear in exactly this form in dozens of places in the source code; the easiest way to change them is to prepare a block of text that replaces the first three lines and use the search-and-replace feature common to most text editors. For example, if we wish to change the parameter statements shown above so that LFY is increased to 5 and the number of rock or soil types (parameter LZN) is increased to 9 , then we begin by making a copy of the three lines of parameter statements and making the desired changes to the copy:

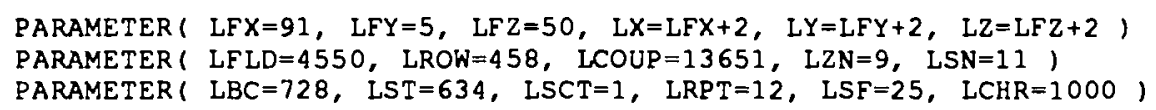

Next, order the text editor to search for occurrences of those original three lines in the file and to replace them with the three new lines. When all occurrences are replaced with the new lines, save the newly parameterized source code file to your working directory (do not overwrite your original source code!).

A time-saving feature of the MSTS Graphical Input is the "Compute Parameters" feature. Once you have finished preparing an input file and have saved it, choose the "Compute Parameters" feature and select the input file of interest. The Graphical Input will compute the minimum required parameters for that input file and display them on screen; more useful yet, it will write the three parameter statements to a file entitled "parameters" in the default directory. Now all you have to do to parameterize the code is open the MSTS source code file and the parameter file with your text editor and use the contents of the parameter file as the text to replace the old parameter statements.

\subsection{MSTS Source Code Machine Specification}

Machine specification involves changing certain commented lines in the MSTS source code into executable statements so that the code is customized for a particular computer. Ordinarily, these lines of code are concerned with obtaining the execuation time and date from the host computer for quality assurance purposes. Because the way in which the computer's clock is accessed is different from one computer to the next, some machine-specific code is necessary. 
Such code has been included for a number of machines in the "msts.f" file, and then "commentedout" so that none of the machine-specific code is active is the distribution "msts.f" file. A special "comment block" name has been reserved for each computer supported by the MSTS code, such as "CMAC" for Macintosh. Here is an example of Macintosh-specific code in MSTS:

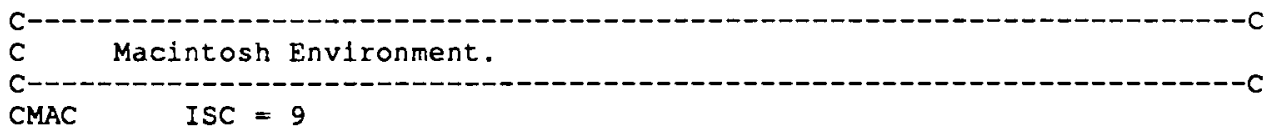

To make the code ready to compile on the Macintosh, remove the "CMAC" comment block:

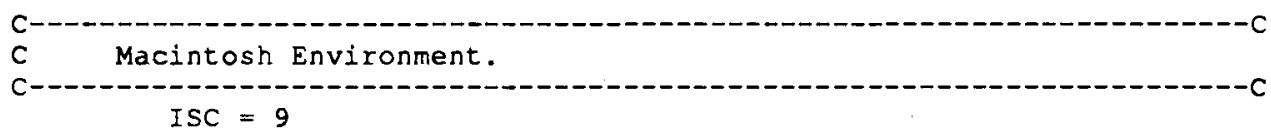

This must be done for all occurences of "CMAC" in the code. The efficient way to do this is to use the search-and-replace feature of your text processor to find all occurences of "CMAC" (or the appropriate comment block for your machine) and replace it with feur spaces.

Table 4.3 lists the machines and associated comment block names that were available in the "msts.f" file at the time this document was written. Other machines may have been added since, so check the source code file if your machine does not appear in Table 4.3.

Table 4.3. MSTS-Supported Machines and Associated Comment Blocks

\begin{tabular}{lcc}
\multicolumn{1}{c}{ Computer } & Comment Block Name \\
\cline { 1 - 1 } Apple Macintosh & & CMAC \\
Sun & CSUN \\
IBM (AIX) & CIBM \\
Stellar & CSTLR \\
HP 9000 & CHP \\
Convex & CNVX \\
Cray & CRAY
\end{tabular}


. 


\subsection{MSTS Quick Tour}

This section leads the new MSTS user through a quick tour of both use of the MSTS Graphical Input and an application of the MSTS code. Following the tour outlined in this section is highly recommended for new MSTS users as a fast way to "come up to speed" on the use of MSTS. The quick tour leads the reader through the steps necessary to create an input file for performing a one-dimensional simulation of vertical water infiltration into a generic sand. This section assumes that the reader is using a Macintosh computer with a FORTRAN compiler installed and has a basic mastery of the Macintosh computing environment. Terms such as "point and click," "double-click," "drag," and "launch" should be familiar to the reader. It is further assumed that the user understands the fundamentals of unsaturated flow in porous media that are simulated in this tour. The quick tour requires approximately 2 hours to complete.

\subsection{Installing MSTS on a Macintosh Computer}

If not already done, install the MSTS source code and the MSTS Graphical Input application on your Macintosh's hard disk. Insert the distribution disk containing the MSTS source code into the floppy disk drive and drag the "msts.f" icon to an appropriate folder in your hard disk. After the file is copied, eject the floppy disk and insert the disk containing the MSTS Graphical Input application. Copy this file to the same folder you put the source code in and eject the second disk.

\subsection{Launching the MSTS Graphical Input}

Launch the MSTS Graphical Input either from the menu (select the icon, then choose Open from the File menu) or by double-clicking the application icon. The MSTS Graphical Input information window (Figure 5.1) will be displayed while the application loads. A new menu bar (Figure 5.2) replaces the Macintosh Finder menu when the Graphical Input is loaded. The menu bar includes the familiar Apple, File, and Edit menu items, plus several menu items that are unique to the Graphical Input menu bar.

\subsection{Creating a New Input File}

To create a new simulation file, select New from the File menu. Specify the input file name and folder using the dialog box (Figure 5.3) that appears next. Enter the name "input" in the box titled "New Input Title" and choose a folder. Click the Save button to continue. When creating a 


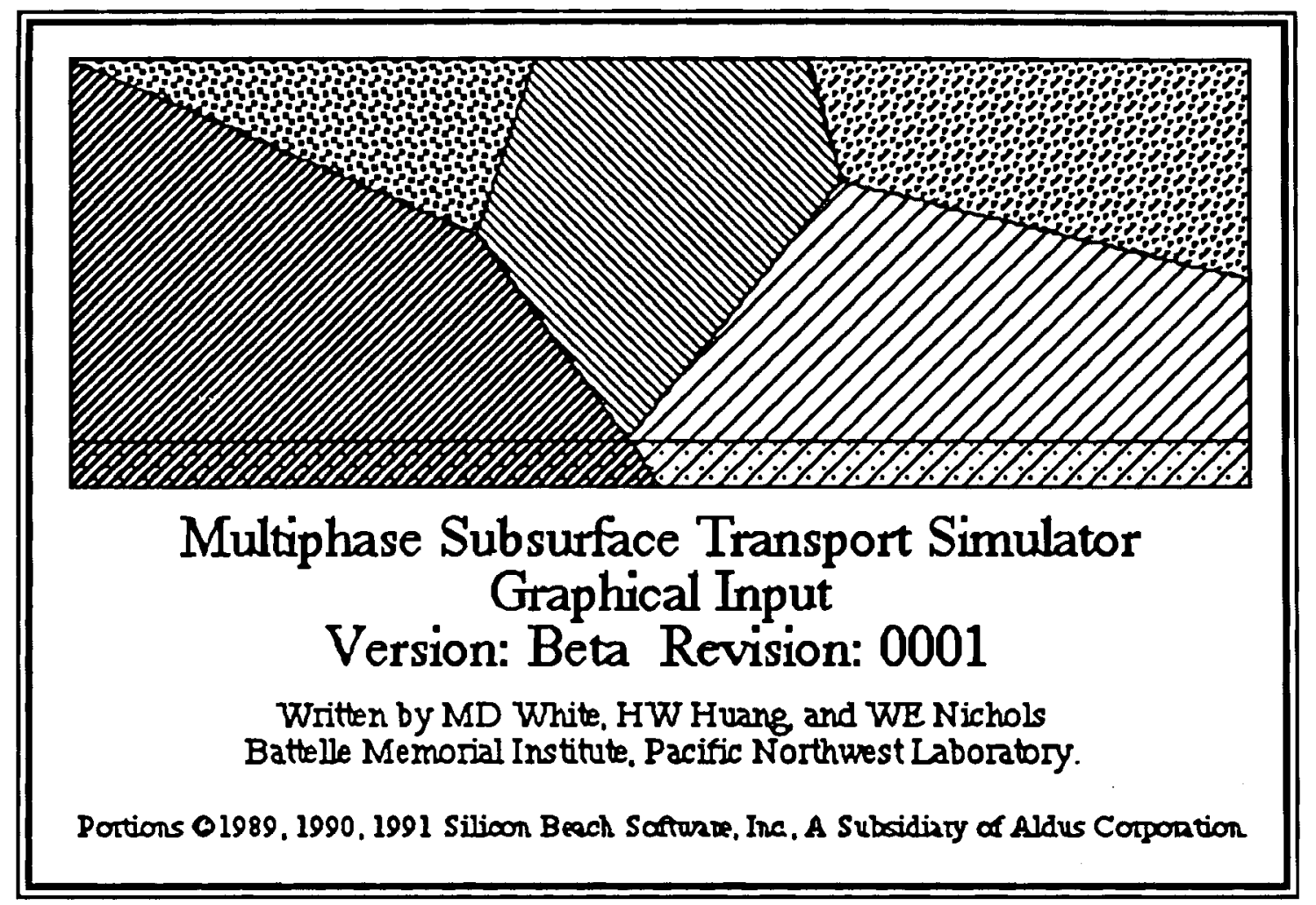

Figure 5.1. Information Window from MSTS Graphical Input

a File Edit Windows Tables contril maneities Candilians Resources

Figure 5.2. Menu Bar from MSTS Graphical Input

new input file, the Graphical Input responds with a dialog box that asks if you wish to initialize all cards (Figure 5.4). If the Yes button is selected, all data-entry fields will be initialized to blank fields or default values. Otherwise, all data fields will contain the data entered most recently since the last initialization (including previous Graphical Input sessions). Individual cards may be initialized by selecting the Initialize item from the File menu while the card is open. Click on the Yes button. A feature of the Graphical Input is the ability to hold the most recent options and user entries in memory until replaced or initialized. This feature can be useful if you are preparing a new input file that is only slightly different from the last one you prepared, and saves recalling the old file before modifying it. To begin with a clean slate, though, initialize all cards.

Next, the Main Menu card will appear, centered on your screen (Figure 5.5). This card will have the input file name on the window bar (top) and two columns of labeled buttons. Each button 


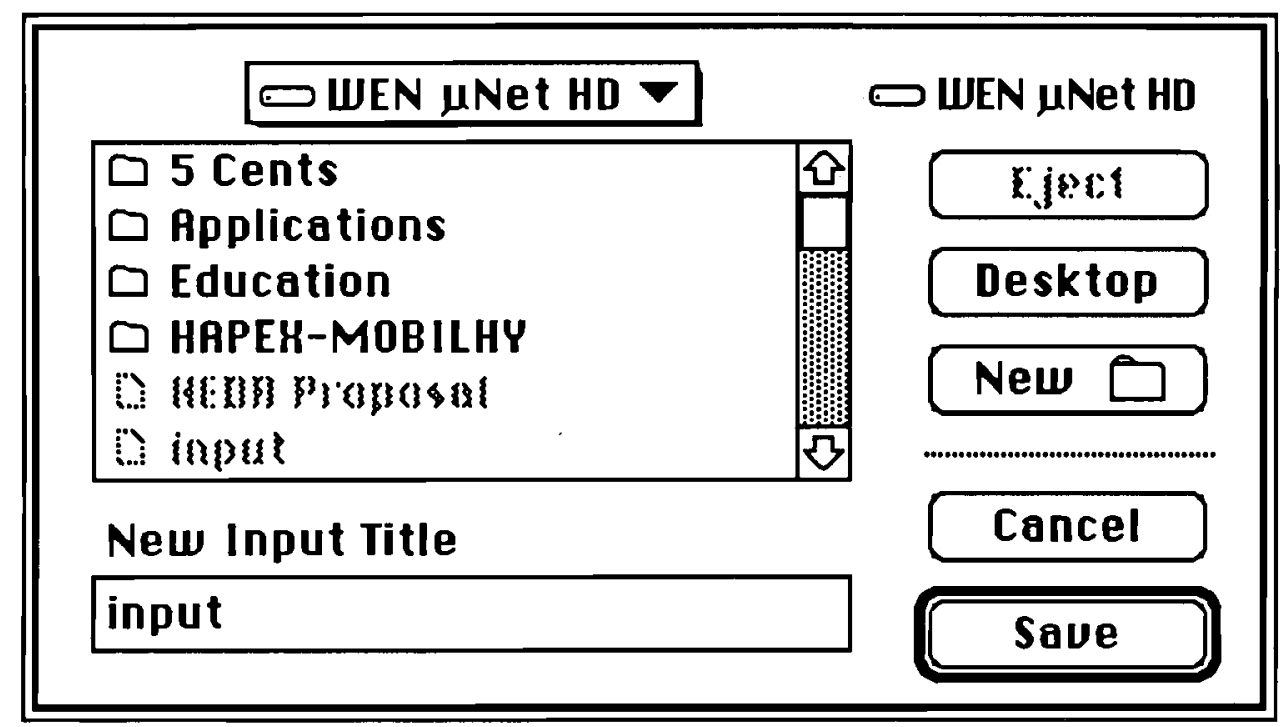

Figure 5.3. File Dialog Box from MSTS Graphical Input

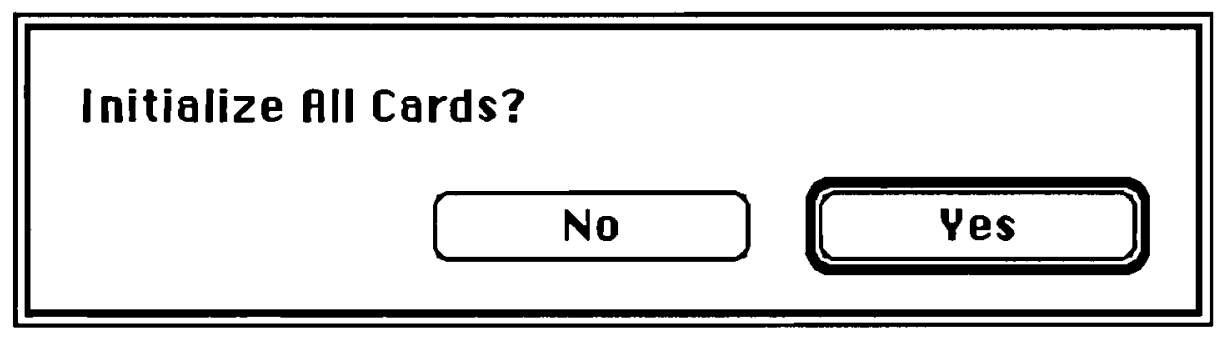

Figure 5.4. Initialization Prompt for New Input Files

corresponds to a particular MSTS input card and when clicked, opens that card. We will begin preparing the simulation input file with the Simulation Title card. Click on the top button of the left column of buttons, labeled "Simulation Title." The card that appears (Figure 5.6) is used to enter record information (e.g., simulation title, user's name and affiliation, file creation date, and notes about the simulation). Complete these fields with your own personal information, using Figure 5.6 as a guide. Clicking the Date button will automatically enter the file creation date and time.

\subsection{Navigating Between Input Cards}

The four arrow icons at the top right of the input card (Figure 5.6) allow you to navigate between input cards. When clicked, the first arrow will always return you to the Main Menu card; the second arrow (pointing left) will open the previous input card in the input card order (that is, 


\section{Multiphase Subsurface Transport Simulator Graphical Input}

\begin{tabular}{|c|}
\hline Simulation Title \\
\hline Solution Schemes \\
\hline Numerical Control \\
\hline Grid Geometry \\
\hline Inactive Nodes \\
\hline Rock or Soil Types \\
\hline Mechanical Properties \\
\hline Hydraulic Properties \\
\hline Thermal Properties \\
\hline Species Properties \\
\hline Soil Characteristics \\
\hline Liquid Permeability \\
\hline Gas Permeability \\
\hline
\end{tabular}

\begin{tabular}{|c|}
\hline Liquid Boundary Cond. \\
\hline Gas Boundary Cond. \\
\hline Uapor Boundary Cond. \\
\hline Energy Boundary Cond. \\
\hline Species Boundary Cond. \\
\hline Sources \& Sinks \\
\hline Initial Conditions \\
\hline Liquid Pathline Trace \\
\hline Output Control \\
\hline Surface Flus Integrator \\
\hline
\end{tabular}

Figure 5.5. Main Menu Card from MSTS Graphical Input

the order in which the cards appear in Table 6.1 of Section 6.0, and in the MSTS "input" file); the third arrow (pointing right) will call the next input card in the input card order; and while the last arrow will open the next-higher-level card returning up the hierarchy of card levels. Click the last arrow to return to the Main Menu card. Reading down the left column of buttons on the Main Menu card, the next button after Simulation Title is Solution Schemes; click on the Solution Schemes button to navigate to that input card.

\subsubsection{Solution Schemes}

The Solution Schemes input card (Figure 5.7) allows you to specify the solution options, the simulation time period, the solution technique, and other control information. Click on the Water 


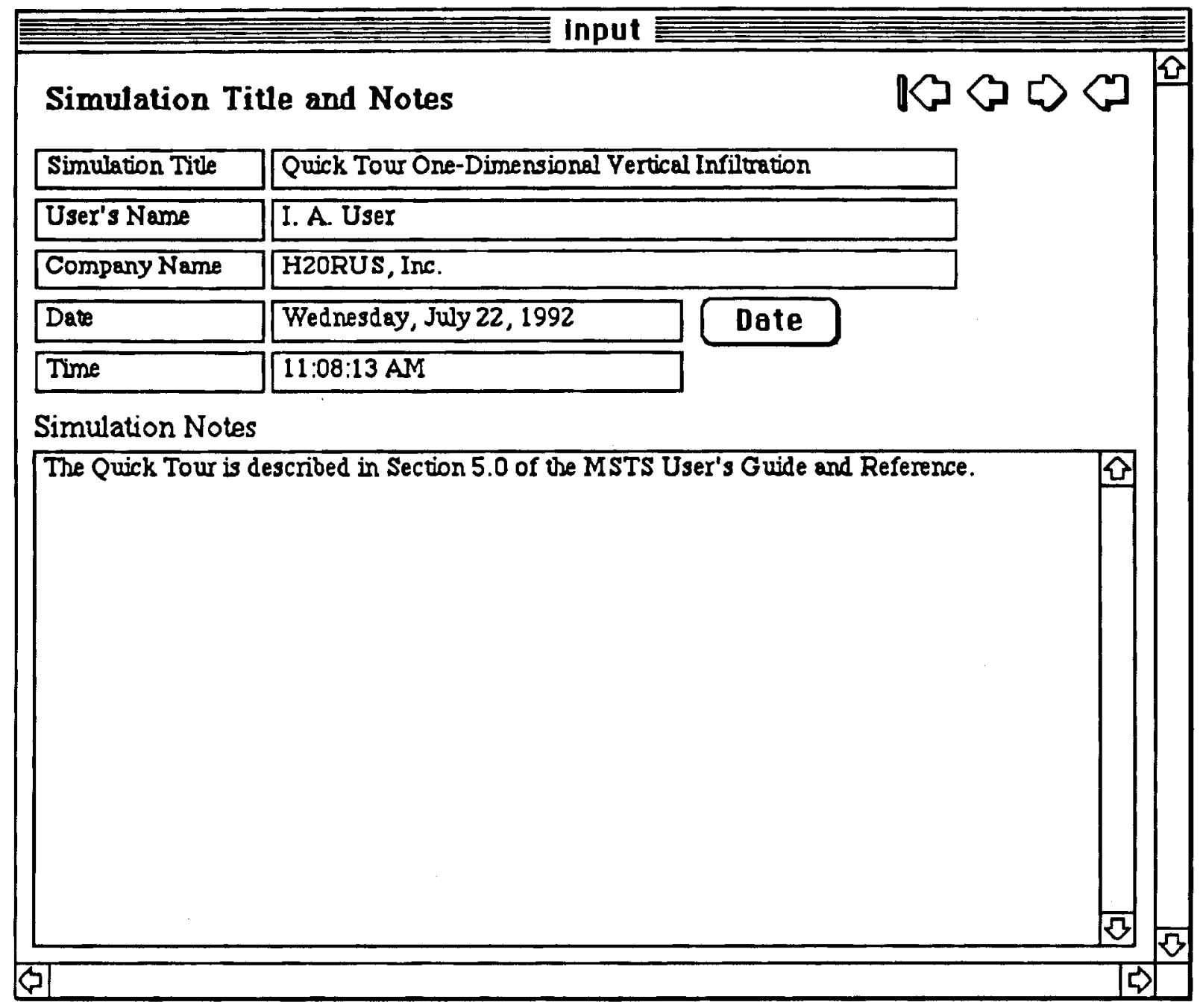

Figure 5.6. Simulation Title Input Card Example

Conservation box (but not the other three, Thermal Energy, Air Conservation, or Species Concentration) to invoke the Water mode (see Section 2.4 for more information on solution methods available). To fill the data arrays in MSTS and examine initial conditions without solving the equations, check the Initial Conditions Only box. The initial conditions option is useful for computing saturations and physical properties from the initial primary variables specified either in the Initial Conditions card or in a restart file. We will avoid that option for now. Because we are starting from the beginning, leave the Restart box unchecked.

To enter the solution limits information, click on the Units button, drag down the button menu, and select " $h$ " (hours). Enter in the respective boxes a time limit of 2.0 hours, an initial 


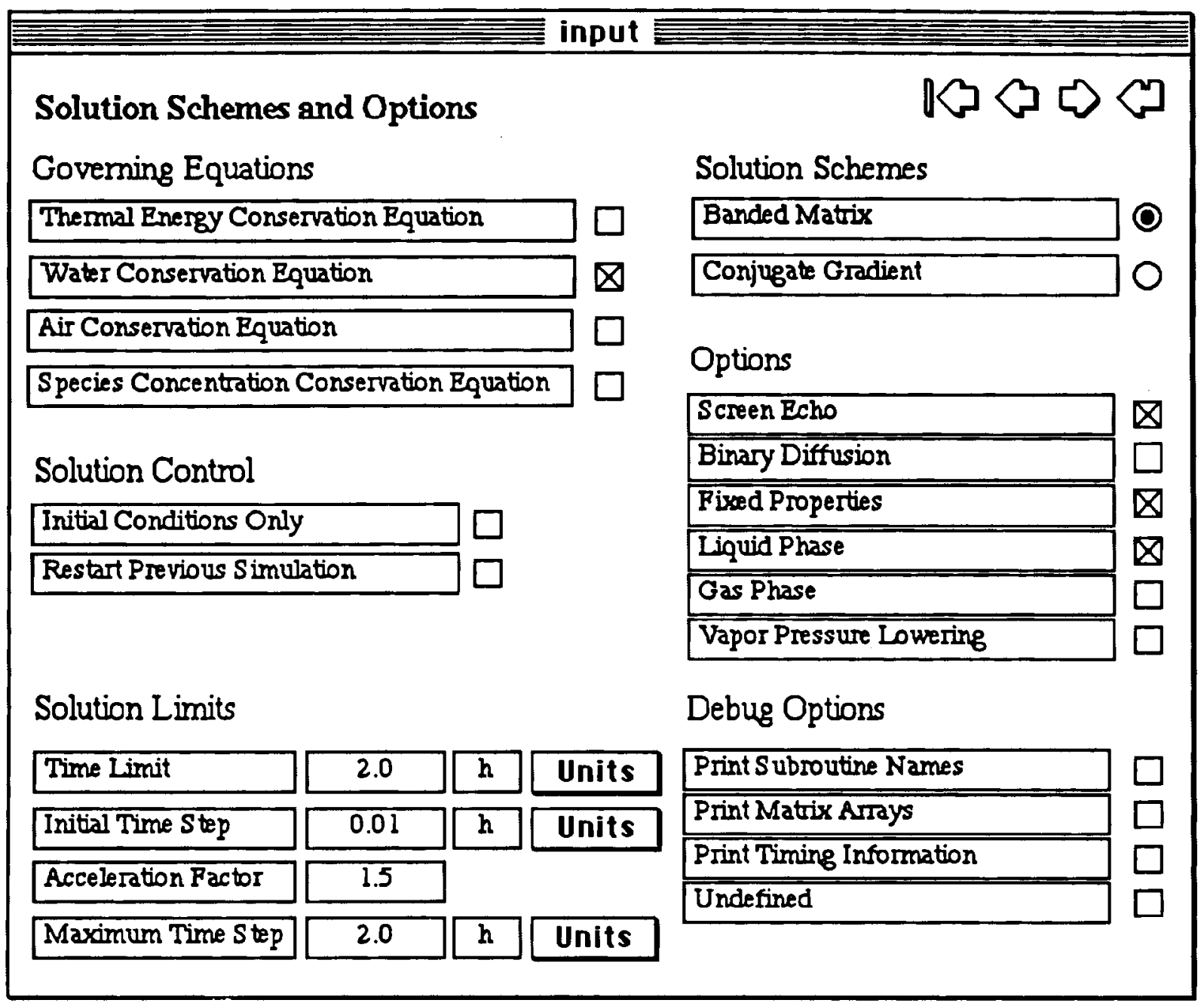

Figure 5.7. Solution Schemes Input Card Example

time step of 0.01 hour, a time-step acceleration factor of 1.5 , and a maximum time step of 2.0 hours. These numbers control, respectively, the time length, the initial time step, the acceleration factor that specifies how fast MSTS will try to increase the time step (a factor of 1.5 would mean a 1-second time step would be increased to 1.5 seconds on the second step, 2.25 seconds on the third step, and so on until a convergence limit is exceeded), and the maximum time step. Because we chose hours as the time unit, all solution limits are expressed in units of " $h$ ".

Under "Solution Schemes," check the Banded Matrix option. This is usually acceptable for most simulations. Refer to Section 4.6 of the MSTS Theory Manual (White and Nichols 1992) for a discussion of the available linear-equation solution schemes. 
"Options" include an assortment of check boxes. Check the Screen Echo box so that MSTS will report reference node information to your computer screen during the simulation execution. Also check the Fixed Properties box, which is used to prevent MSTS from recomputing certain physical properties (e.g., fluid density) each time step, thus achieving a faster execution for problems where these properties do not vary. The Fixed Properties option should not be invoked for problems involving variable physical properties (e.g., density-driven flow through porous media). The Liquid Phase box was automatically checked for you when you activated the water conservation goveming equation; leave this box checked. Because we are not considering gasphase migration of water vapor, do not check the Gas Phase box. Do not check any of the "Debug Options" either, these are not needed for our quick tour.

\subsubsection{Numerical Control}

Click the third arrow at the top right-hand comer of the Solution Schemes input card to navigate to the Numerical Control card. Enter 8 in the Maximum Iterations per Step field. This number limits the number of Newton-Raphson iterations per convergence attempt. Select the Maximum Residual convergence mode from the next button. Set the "CPU Limit" and "Wallclock Limit" to 1 hour each (remember to set the time units to hours with the Units buttons). Finally, set the convergence criteria to $1.0 \times 10^{-6}$ (enter this as 1.0E-06) for the water equation and choose the Geometric Mean option for property averaging for the water equation. The case of the "e" exponent indicator is unimportant for MSTS input, as are leading zeros (e.g., 1.0E-06 is the same as 1.e-6). Convergence criteria and averaging techniques may be set for the other equations as well but are not needed because only the water conservation equation will be solved. Navigate to the next card (Grid Geometry).

\subsubsection{Grid Geometry}

The quick tour problem requires a one-dimensional, vertical model domain. Begin constructing the grid by checking that the coordinate system is Cartesian; if the radio button next to the "Cylindrical Grid" on the right side of the Grid Geometry input card (Figure 5.8) is highlighted, check "Cartesian Grid" instead. Enter numbers in the fields under the "Number of Nodes" portion of the input card so that there is 1 node in the $x$-direction, 1 node in the $y$-direction, and 20 nodes in the $z$-direction. Then indicate for "Grid Dimensions" that the $x$-and $y$-dimensions are $1 \mathrm{~cm}$, and the $z$-dimension is $20 \mathrm{~cm}$. The datum elevation and the grid bottom elevation should both be $0 \mathrm{~cm}$. At this point we could enter the node positions manually, by entering the values and accepting them, but a faster way to construct the grid for uniformly spaced nodes is to click the Uniform button. The Graphical Input will generate the node coordinates and record them in the 


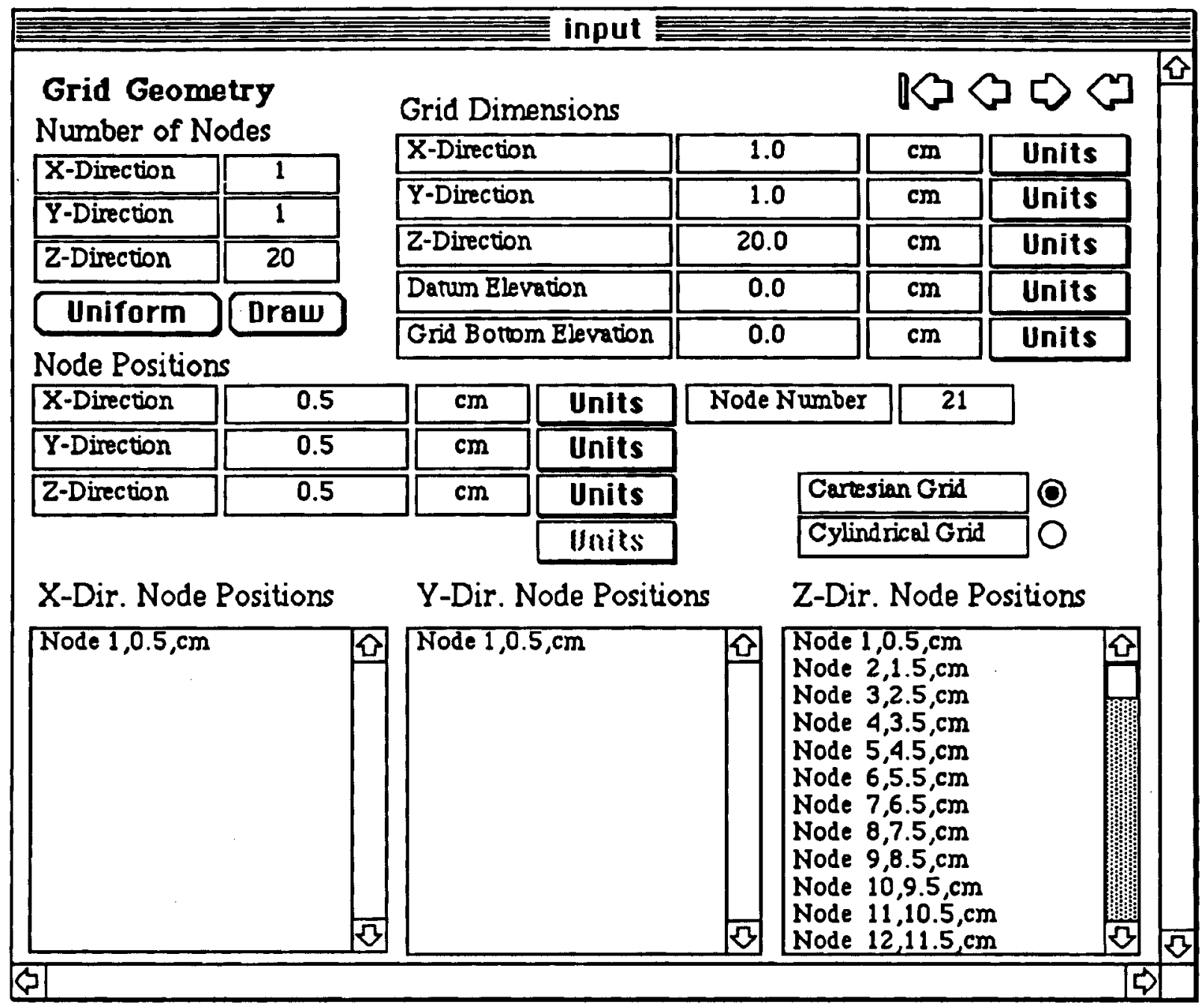

Figure 5.8. Grid Geometry Input Card Example

appropriate fields at the bottom of the Grid Geometry card (see Figure 5.8). There are no inactive nodes in this problem, so navigate to the Rock or Soil Types card.

\subsubsection{Rock or Soil Types}

Our simulation domain comprises only one type of porous material, which we term generic sand. Click in the blank field next to the box titled "Rock Name" and type "Generic Sand" to identify the soil. If this medium filled only a portion of the domain, we would enter the node number ranges in $x, y$, and $z$ to indicate its extent. Because the generic sand extends over the whole domain, we simply click the Entire Domain button to have the Graphical Input automatically fill these fields with the maximum node range. Note that use of the Entire Domain button requires that 
the Grid Geometry card already be completed. Now the soil type is specified but not recorded; press $\Delta$ (command and A keys together) or select Accept from the Edit menu to record the rock or soil type in the Rock List (more about that later). After the entry is recorded, a line will appear in the scrolling box at the bottom of the card that looks like this:

Generic Sand, 1, 1,1,1,1,20,

Navigate to the next card (Mechanical Properties).

\subsubsection{Mechanical Properties}

Choose Rock List from the Windows menu. A floating window will appear (Figure 5.9) that contains the names of all rock types you have recorded. At this point, only one name will appear: "Generic Sand." If you double-click on "Generic Sand" in the Rock List, the name will be placed in the Rock Type field of the Mechanical Properties card (or any other input card with a field entry titled "Rock name"). You may use the Rock List to pick rock or soil types for any card that requires one. Close the Rock List. Enter total porosity of 0.40 , diffusive porosity of 0.50 , and effective porosity of 0.30 . Ignore the tortuosity option fields; these are unnecessary for this tour. Now accept the entry (press [D or select Accept from the menu). Have you noticed the authors' mistake yet? Diffusive porosity cannot exceed total porosity; we have reversed these numbers, so the two must be corrected. This may be done in two ways. One is to select the record line that was to be corrected (click once on the line and it will highlight), and delete it by pressing [D] or selecting Delete from the Edit menu. Then correct the entry and accept it again. The other way is to replace the record line by correcting the Total Porosity and Diffusive Porosity fields, then highlight

the line and press $[\mathbb{g}$ (or choose Replace from the Edit menu with the line highlighted). Using either method, correct the porosity entries for generic sand. The Accept, Delete, and Replace functions are similar for all input cards in the Graphical Input. Navigate to the next card (Hydraulic Properties).

\subsubsection{Hydraulic Properties}

By now you should be able to see the pattern for input card preparation. Test your ability by picking the rock type and entering a $z$-direction hydraulic conductivity of $1.0 \times 10^{-2} \mathrm{~cm} / \mathrm{s}$ (enter as 1.0E-02). Porous media transmissivity may be expressed with either a hydraulic conductivity at $20^{\circ} \mathrm{C}$ or an intrinsic permeability. Units preceded by "hc:" are for hydraulic conductivity and are expressed as length per time $\left(\mathrm{L} \mathrm{t}^{-1}\right)$, whereas intrinsic permeability units are measured in length squared (L2). Accept your entry and navigate to the Soil Characteristics card (we will skip the 


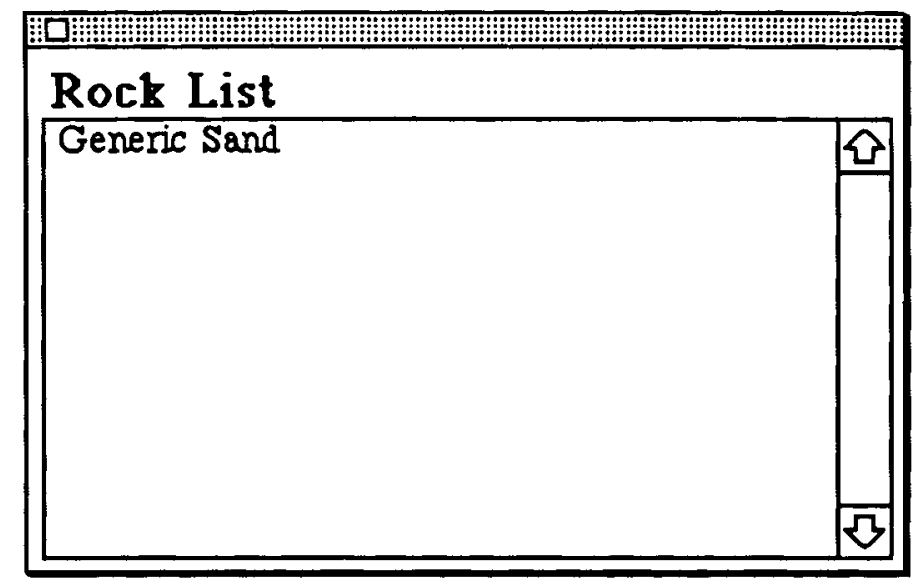

Figure 5.9. Rock List from MSTS Graphical Input

Thermal Properties and Species Properties cards because the quick tour problem is not concerned with heat transfer or species transport).

\subsubsection{Soil Characteristics}

Select Generic Sand from the Rock List and choose van Genuchten from the Moisture Retention button menu. Enter an $\alpha$ (alpha) parameter value of $0.051 / \mathrm{m}$, an $n$ parameter value of 1.5 , and a maximum tension head value of $1.0 \times 10^{5} \mathrm{~m}(1.0 \mathrm{E}+5)$. The online help feature of the Graphical Input may be used to inquire about the van Genuchten function. To invoke this feature, press $[\mathbb{D}$ or choose Help from the Window menu. The help window will appear, displaying information related to the input card currently open. Click on the Find button, type "van Genuchten" in the Find Info... field, and then press the RETURN key. The help window will display the van Genuchten equations (Figure 5.10). Information about many other topics may be found through online help. When finished, click the Ok button to close the help window. Accept your input entry and navigate to the next card (Liquid Relative Permeability).

\subsubsection{Liquid Relative Permeability}

Choose Generic Sand from the Rock List. Select the Mualem w/ van Genuchten option to compute relative permeability, and enter the value of the parameter $m$. If you do not know how to compute the value of $m$, check the help window on van Genuchten again where the value of $m$ is defined (remember, $n=1.5$ for this problem; use the equation you see in the help screen to compute $m$ and enter that value). Finally, enter a minimum liquid relative permeability of $1.0 \times 10^{-10} \mathrm{~m}$ (1.0E-10). Accept your entry and navigate to the Liquid Boundary Conditions card. 


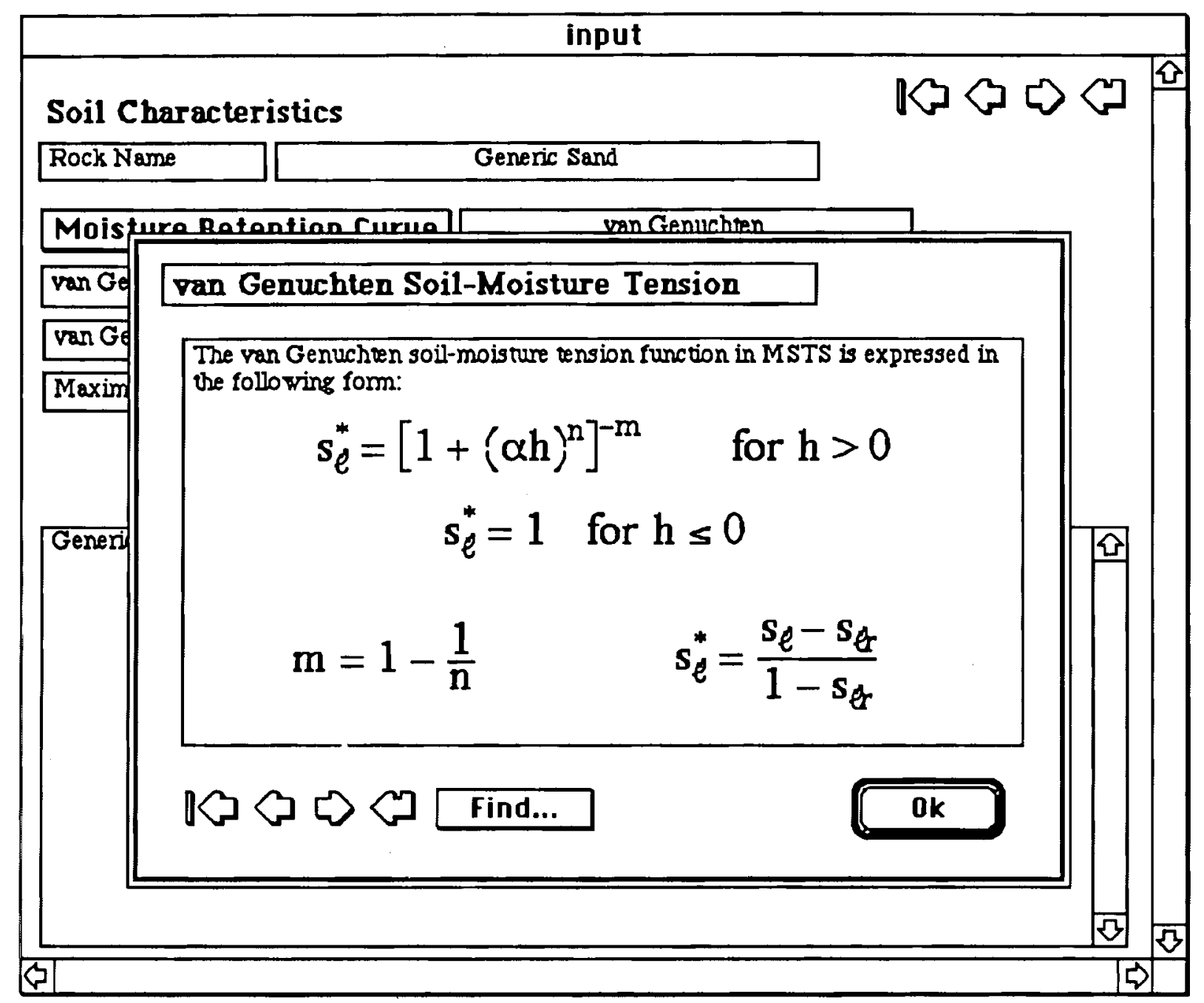

Figure 5.10. Example of MSTS Graphical Input Interactive Help Feature

\subsubsection{Liquid Boundary Conditions}

The quick tour problem involves a fixed water recharge of $1.0 \mathrm{~cm} / \mathrm{h}$ at the top of the column and saturated conditions at the column bottom. To specify the top boundary condition as constant recharge, choose Z-Direction: Top Surface from the Orientation Index button menu and click the Entire Surface button. Choose the Neumann @ Boundary condition from the Boundary Type button menu. The mass flux is computed by multiplying the volumetric recharge of $1.0 \mathrm{~cm} / \mathrm{h}$ by the density of water $\left(998.32 \mathrm{~kg} / \mathrm{m}^{3}\right.$ at $\left.20^{\circ} \mathrm{C}\right)$, as follows:

$$
\text { Mass Flux Rate }=\left(1.0 \frac{\mathrm{cm}}{\mathrm{h}}\right)\left(\frac{1 \mathrm{~m}}{100 \mathrm{~cm}}\right)\left(\frac{1 \mathrm{~h}}{3600 \mathrm{~s}}\right)\left(998.32 \frac{\mathrm{kg}}{\mathrm{m}^{3}}\right)=2.773 \times 10^{-3} \mathrm{~kg} / \mathrm{m}^{2} \mathrm{~s}
$$


Type $2.773 \mathrm{E}-3$ in the Field Variable field to represent the liquid mass flux and enter $998.32 \mathrm{~kg} / \mathrm{m}^{3}$ as the liquid density (remember to select the appropriate units!). The air mass fraction may be neglected for this problem. Accept the entry. For the lower boundary, choose the appropriate index (Z-Direction: Bottom), click the Entire Surface button, and select "Saturated @ Boundary" as the boundary type. Enter the same liquid density as before and accept this entry also: Check that there are two lines in the scrolling box at the bottom of the input card that look like this:

2-Direction: Top Surface, Neumann Boundary, $2.773 \mathrm{E}-3, \mathrm{~kg} / \mathrm{m}^{\wedge} 2 \mathrm{~s}, 998.32, \mathrm{~kg} / \mathrm{m}^{\wedge} 3,1,1,1,1,20,20$

Z-Direction: Bottom Surface, Saturated Boundary, ., 998,32, kg/m^3, , 1, 1, 1, 1, 1, 1

The liquid boundary conditions are now prescribed. Because the quick tour problem does not require boundary conditions for vapor, gas, energy, or species transport and there are no sources or sinks specified for this problem, we will skip the other boundary condition cards and the Sources and Sinks card. Navigate to the Initial Conditions card.

\subsubsection{Initial Conditions}

The initial state for hydrologic conditions can be completely defined by specifying any pair of the following field variables: gas pressure, liquid pressure, and liquid saturation. The unspecified field variable will by computed by MSTS from the water-retention characteristic function. Choose the option "Liquid Saturation \& Gas Pressure" from the button menu labeled "Initial Saturation Option" to indicate how initial conditions will be specified for this problem. Specify the first field variable by choosing "Gas Pressure" from the button menu labeled "Field Variable" and click the Entire Domain button to specify the nodes to be initialized. The initial gas pressure throughout the domain will be atmospheric, so enter 101325.0 into the Gas Pressure box and select "Pa (abs)" from the Unit button menu. Leave the gradients at zero (uniform initial conditions). Accept this entry and observe the new line added to the scrolling box below:

Gas Pressure, 101325.0, Pa (abs), 0.0,1/m,0.0,1/m,0.0,1/m,1,1,1,1,1,20,

The liquid saturation is specified in the same way; change the field variable to "Liquid Saturation" using the Field Variable button menu and enter 0.50 for the liquid saturation. The entire domain is still set from the liquid pressure entry, so accept this entry and a second line is recorded:

Liquid Saturation, $0.50,0.0,1 / \mathrm{m}, 0.0,1 / \mathrm{m}, 0.0,1 / \mathrm{m}, 1,1,1,1,1,20$,

These two lines together set the initial hydrologic conditions for our problem. We have not specified an initial temperature, so MSTS will initialize the domain to $20^{\circ} \mathrm{C}$. Navigate to the Output Control card. 


\subsubsection{Output Control}

The Output Control card provides flexibility in the type and quantity of output produced by MSTS during a simulation. Three scrolling field boxes appear on the left side of the Output Control card (Figure 5.11) that display the field variables to be recorded to the three output devices: the output file, the plot file, and the reference node output. The reference node is limited to a maximum of seven field variable outputs. The output and plot files may contain any number of field variables. To modify the field variables in each scrolling box, click on the radio button corresponding to the output device and a listing of possible field variables will appear. Click the check box next to each desired field variable to select that variable for output (click again to deselect any you do not want) and click the Ok button when finished. Practice now by choosing liquid pressure, liquid saturation, total hydraulic head, volumetric moisture content, and $z$-direction liquid velocity for all three output devices (output, plot, and reference node). We will want output for the whole problem, so click the Entire Domain button and click the Z-X Planes radio button to indicate the orientation for output. It is possible to request output at several simulation times, but we will settle for the final condition at the end of the simulation: enter 2.0 and select " $h$ " as the time unit for field variable output times. Accept this entry. Up to four reference nodes can be monitored during a simulation execution. We will use one for this tour, enter 1 for $x, 1$ for $y$, and 20 (the top) for $z$. This causes the values to be displayed at every time step for all of the field variables chosen above for node $(1,1,20)$.

\subsection{Saving an Input File}

To save the completed input file, press $[\underline{[5}$ (or select Save from the File menu). Make sure that you use the name "input" for the saved file. While the file is being written, a "Writing Input File" message will be displayed indicating the recording progress. Although you were not directed to do so in this tour, it is wise to save the input file frequently while you develop it with the Graphical Input to prevent loss in case of a power failure, a machine freeze, or other interruption that could lose all entries to that point.

\subsection{Parameterization and Machine Specification}

Although you have an input file now, you must still determine how large the parameters must be in the MSTS code. The Graphical Input provides a quick way to do this: select "Compute Parameters" from the Resource menu. The Graphical Input will prompt you to choose an input file: 


\section{Output Control}

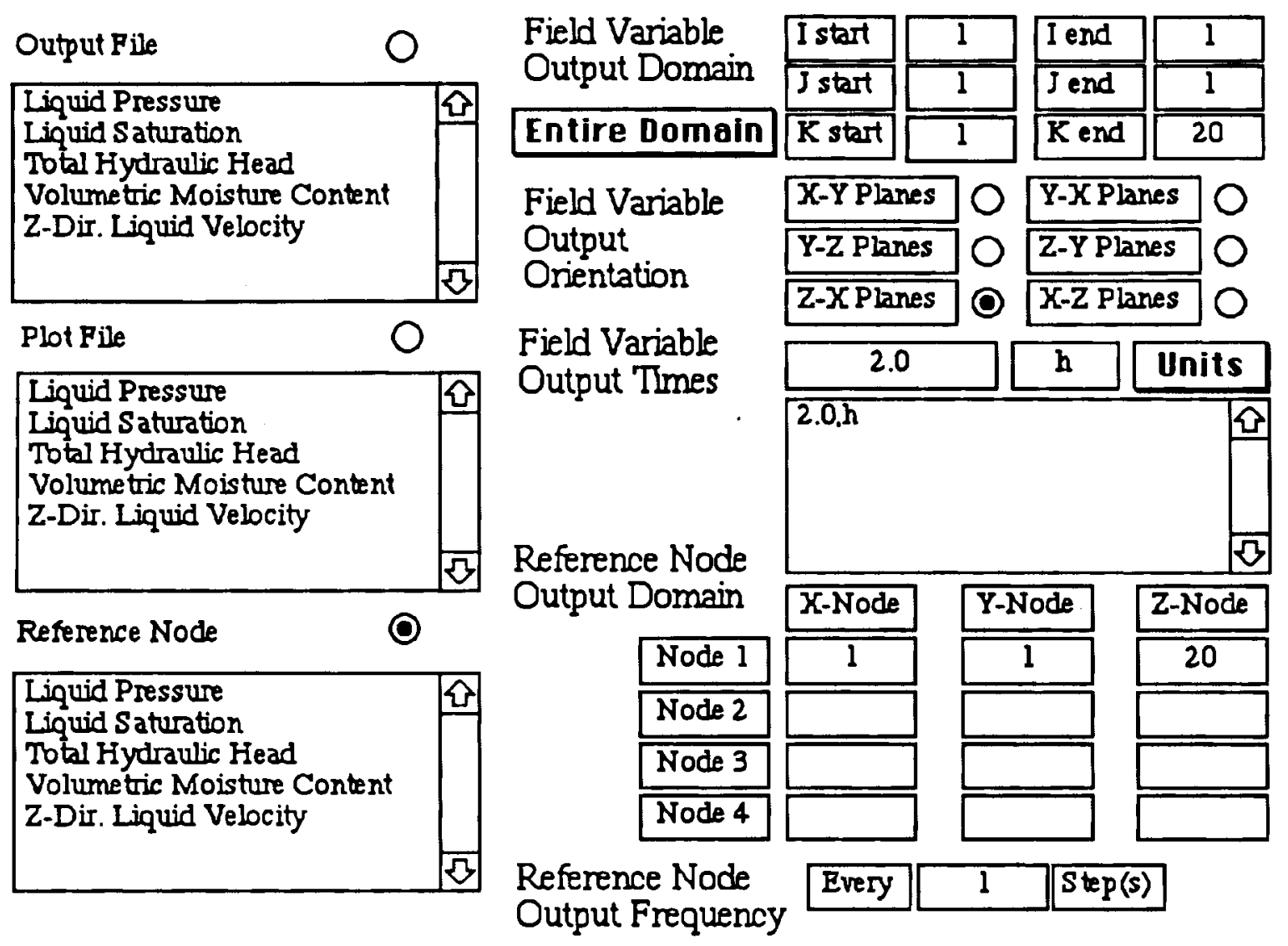

Figure 5.11. Output Control Input Card from MSTS Graphical Input

pick the file "input" that you just saved. The Graphical Input will proceed to read the input file and compute the minimum size for each MSTS parameter (refer to Section 4.0 for details on parameters) for this input file. The results will be displayed on the screen (Figure 5.12) and recorded in the default directory in the file "parameter". You are now finished using the Graphical Input; close it by clicking the Ok button on the parameter display, then choosing Quit from the File menu. Choose No when prompted if you wish to save the input file (you have made no changes since saving it last). With this, your Graphical Input session is closed.

Next you must parameterize the MSTS code. Using your preferred text editor, open the file "msts.f" and locate the first block of parameter statements (within the first 100 lines of code). This parameter block is repeated throughout the code in exactly the same way, so we can use the search- 


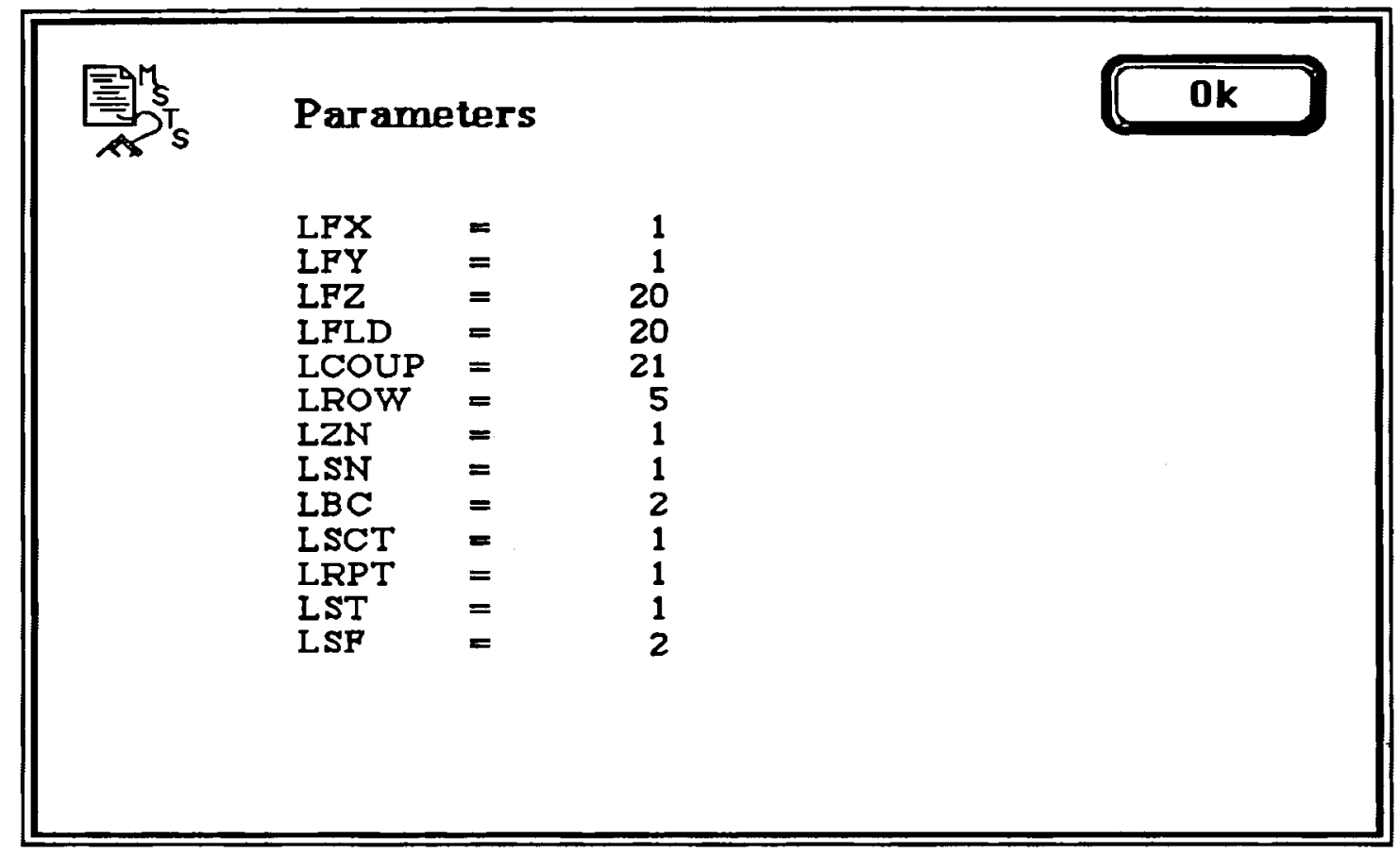

Figure 5.12. Example of the "Compute Parameters" Resource of the MSTS Graphical Input

and-replace feature of your text editor to make parameterization easy. Search for all occurrences of the first three (not all five) parameter statements, and replace them with the three parameter statements you will find in the file "parameter" that was written by the Graphical Input.

To make the code machine-specific, search for a set of comment lines that pertain to your code and delete them. Assuming you will run the MSTS code for this tour on your Macintosh computer, search for all occurrences of "CMAC" and delete these. This will make active statements out of the time and date functions MSTS requires that vary from one computing environment to another. Other machines have different comment lines to alter: "CRAY" for the Cray computer, "CSUN" for a Sun workstation, and so on (see Section 4.0 for a complete listing of these). When finished deleting the "CMAC" occurrences, save the "msts.f" file. (Important: be careful you save an original, unaltered copy of the "msts.f" file!) The code is now parameterized and specific to your computer. It is important to save the original "msts.f" file because making it machine-specific by altering a fresh copy of the original each time is easier than trying to restore one set of comments and deleting another (e.g., restoring all the "CMAC" occurrences and deleting all the "CSUN" occurrences to prepare for a simulation on a Sun workstation is more difficult than merely deleting the "CSUN" occurrences on a new copy). 


\subsection{Running a Simulation}

Ensure that the "input" and "msts.f" files you have prepared are in the default directory you wish to use for the simulation. Compile the "msts.f" using the FORTRAN compiler you have available. Then invoke the application (executable file); no other action is required. MSTS will read the file "input" and follow its instructions.

\subsection{Examining Output}

General simulation output is contained in the "output" file, which is in ASCII format and may be viewed with the text editor of your choice. The output file contains a unique run-identification number and the time and date of the run as well as an "echo" of the information read from the input file. Check the first part of the output file to ensure that the input information repeated there is what you entered using the Graphical Input. Next, the output file records the reference node information just as it appeared on the screen during the simulation execution. Finally, the field variables you requested in the Output Control card of the input file are recorded.

Most often you will wish to graph the results of your simulation or prepare images (contour plots or raster images) of the results. The "plot" file generated by MSTS makes this easier to do. Any field variables separately requested for the plot file are recorded in a format more conducive to plotting tasks. Following some header information, the names of the variables in the plot file are listed (one per line), followed by a listing of the field variables in increasing node order. The node number and $x-, y$-, and $z$-coordinates of the node are always provided in the first four columns and the node volume in the fifth column.

\subsection{More Sample Problems}

Additional sample problems can be found in Section 8.0. The samples include single-node evaporation and condensation tests, a heat pipe problem, flow from hot two-phase conditions, vertical and horizontal infiltration problems, and an unsaturated transport problem. 


\subsection{Input File Structure}

Control and model parameter specification is accomplished in MSTS through the "input" file. An "input" file may be prepared either by using the MSTS Graphical Input (see Section 3.0 for details) or by using a text editor and complying with the MSTS input file format requirements. This section provides detailed information for each card in an MSTS input file and is intended as a reference. The typical MSTS user will commonly use the Graphical Input and therefore not need to be intimately familiar with all the details provided in this section but may refer to them as needed.

This section is grouped by card function (e.g., all of the cards that are used to specify boundary conditions are together), and the cards are organized in the same order as in an MSTS input file. Table 6.1 summarizes all of the input cards contained in an MSTS input file in this order.

The "input" file itself must be an ASCI file and hence is portable between computing environments. Therefore, a user may prepare an "input" file on a personal computer, transfer the file to a mainframe, and run the simulation. The syntax rules used in this section are presented in Table 6.2. MSTS avoids case-sensitive errors by converting all input file contents to lower case as the input is received by the code. Very little other error checking is performed on the input; hence close attention to the format requirements presented in this section is necessary. The easiest way to avoid format errors is to use the MSTS Graphical Input to prepare input files, as that application is specifically designed to comply with all of the format requirements contained in this section. 
Table 6.1. MSTS Input Cards

Input Card

Simulation Title

Solution Schemes

Numerical Control

Grid Geometry

Inactive Nodes

Rock or Soil Types

Mechanical Properties

Hydraulic Properties

Thermal Properties

Species Properties

Soil Characteristics

Liquid Relative Permeability

Gas Relative Permeability

Liquid Boundary Conditions

Gas Boundary Conditions

Vapor Boundary Conditions

Energy Boundary Conditions

Information Specified

identification of simulation, user, notes, file creation time and date solution scheme, governing equations, simulation time control iteration limits, convergence criteria, averaging techniques coordinate system, physical extent, number and location of nodes location of noncomputational nodes identification of rock or soil types and node assignment porosities and tortuosities

hydraulic conductivities (or permeabilities)

thermal conductivities, rock or soil density, heat capacity identification of species, decay rates, coefficients moisture-retention characteristics, tension head limits unsaturated liquid relative-permeability parameters gas relative-permeability parameters

type, extent, and magnitude of boundary conditions for liquid water type, extent, and magnitude of boundary conditions for gas type, extent, and magnitude of boundary conditions for vapor type, extent, and magnitude of boundary conditions for thermal energy

Species Boundary Conditions type, extent, and magnitude of boundary conditions for species mass

Initial Conditions

Sources and Sinks

Liquid Pathline Trace

Output Control

Surface Flux Integrator specification of field variable initial values location, temporal variation, and strength of sources and sinks liquid pathlines for which pathline and travel times are required field variables to record in output files planes to report fluxes of mass or energy as a function of time 
Table 6.2. Input Reference Conventions

Notation

Line 1

Line $n$

$\sim$

I, J, K

$[x \mid y]$

\{ alpha \}

\{ alpha-numeric \}

$\{$ integer \}

$\{$ real \}

( text )

text

"text"
Interpretation

The first line in an input card following the card name.

One of $\mathrm{N}$ line(s) in an input card, where $\mathrm{N}$ is declared in the beginning of the input card and $1 \leq n \leq N$.

A tilde always precedes the name of an input card and signals the input modules of MSTS to the beginning of a new input card.

Commas separate individual entries in a line within an input card. A terminating comma is usually required at the end of each line (inclusion of a terminating comma where it is not required does not adversely affect input).

Indices for node ranges in the $x-, y$, and $z$-directions for Cartesian coordinate systems, or indices for node ranges in the $r_{-}, \theta_{-}$, and $z$ directions for cylindrical coordinate systems.

Square brackets indicate a list of options separated by vertical lines. Only one of the available options may be used and must be written precisely as shown in the option list except that the case of characters is unimportant ( $\mathrm{Aa}$, aa, and $\mathrm{AA}$ are all equivalent).

Braces enclosing the word "alpha" indicate field inputs of alphabetic characters.

Braces enclosing the words "alpha-numeric" indicate field inputs of alphabetic and numeric characters.

Braces enclosing the word "integer" indicate field inputs of integer numbers.

Braces enclosing the word "real" indicate field inputs of real numbers.

Text within parentheses gives informal directions on what type of line, or group of lines, may be used in an input card.

Italicized text defines input options.

Text within the quotation marks is to be typed exactly as shown, except that the case of characters is unimportant (Aa, aa, and AA are all equivalent). 
Table 6.2. (Contd)

Notation

text
Interpretation

Courier typeface is used to display text from any computer file, such as an input file. In some cases, a line in a computer file is wider than can be displayed within the margins of this document, in which case the additional text is shown on subsequent indented line(s) to signify that it is a continuation of the same line. To illustrate:

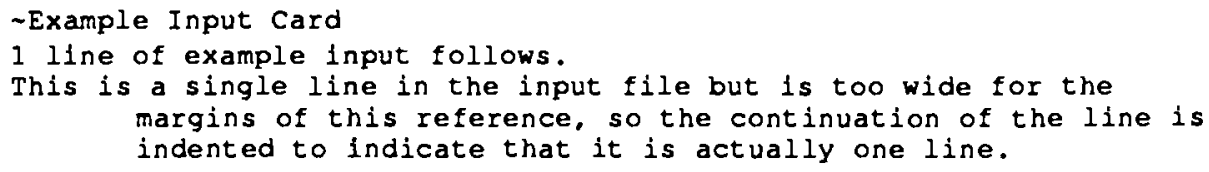




\subsection{Simulation Documentation and Control}

Simulation documentation and control are accomplished with the Simulation Title, Solution Schemes, and Numerical Control cards. The first card provides a way to identify each "input" file by title, user, date and time of creation, and MSTS version name and revision number. The Solution Schemes card is used to specify which combination of the four governing equations (thermal energy conservation, water conservation, air conservation, and species mass conservation) is to be solved, the period of time to be simulated, and the solution scheme (e.g., banded matrix). The Solution Schemes card also provides control over other options like debugging output, screen echoing during a simulation, binary diffusion, etc. The Numerical Control card is used to enter convergence criteria, averaging options, computer time limits, and iteration limits. 


\subsubsection{Simulation Title Card}

\section{Function}

The Simulation Title card is used to specify information that uniquely identifies an "input" file, including the title of the simulation, the user's name and affiliation, the "input" file creation date and time, and any notes the user may enter.

\section{Format}

Line $0 \quad$ " Simulation Title and Notes"

Line 1 Version Name \{ alpha ( 4 characters ) \}, Revision Number, [ integer ( 4 digits ) \}

Line 2 Simulation Title \{ alpha-numeric \},

Line 3 User Name [alpha-numeric \},

Line 4 Company Name \{ alpha-numeric \},

Line 5 "Input" File Creation Date ( alpha-numeric ( 20 characters maximum ) \},

Line 6 "Input" File Creation Time \{ alpha-numeric ( 20 characters maximum ) \},

Line $7 \quad$ Characters \{ integer \} "character(s) of simulation title notes follows."

Line(s) $8+\quad$ Simulation Title Notes \{ alpha-numeric ( 80 characters per line, no returns ) \}

\section{Comments}

The version name and release number provide positive identification of the particular MSTS code used, preventing use of inconsistent MSTS Graphical Input and MSTS code versions. The version name (e.g., "beta") and release number (e.g., "0001") pertain to both the MSTS code itself and the MSTS Graphical Input. The MSTS Graphical Input will accept only files that have the same version name and release number.

The Simulation Title field is a short, one-line description or title that will allow the user to quickly recognize which simulation is controlled by this "input" file. The User Name and Company Name fields are provided for additional background information. The Simulation Title Notes field provides a space to enter a more extensive description of the "input" file.

\section{Examples}

In this example a vertical infiltration problem is identified. The input card belongs to a file created with the beta version of MSTS, release 0001 . The file creation date and time are indicated, 
and a short description of the problem is included in the Simulation Title Notes field.

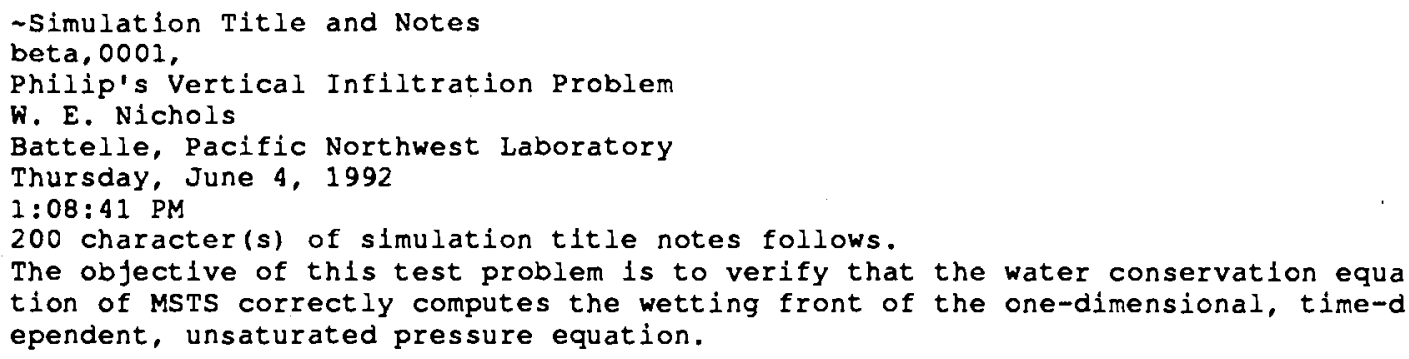




\subsubsection{Solution Schemes Card}

\section{Function}

The Solution Schemes card is used to invoke restart options, control simulation time variables, select a matrix solution scheme, specify which governing equations to solve, choose secondary process options, and invoke debugging assistance options.

Format

Line 0 " Solution Schemes"

Line 1 Initial Conditions Only [ true I false ],

Restart [ true I false ],

Restart from Time Zero [ true | false ],

Line 2 Matrix Solution Scheme [ banded matrix | conjugate gradient ],

Line 3 Energy Equation [ true | false ],

Water Equation[ true | false ],

Air Equation [ true I false ],

Species Concentration Equation [ true I false ],

Line 4 Simulation Time Limit \{real \},

Units $[s|\min | \mathrm{h} \mid$ day $|\mathrm{wk}| \mathrm{yr}]$,

Line 5 Initial Time Step \{ real \},

Units $[s|\min | \mathrm{h} \mid$ day $|\mathrm{wk}| \mathrm{yr}]$,

Line 6 Time Step Acceleration Factor $\{$ real $\}$,

Line 7 Maximum Time Step \{ real \},

Units $[s|\min | \mathrm{h} \mid$ day $|\mathrm{wk}| \mathrm{yr}]$,

Line 8 Screen Echo [ true I false ],

Binary Diffusion [ true I false ],

Fixed Properties [ true | false ],

Liquid Phase [ true I false ],

Gas Phase [ true I false ],

Vapor Pressure Lowering [ true I false ],

Line 9 Print Subroutine Names [ true I false ], Print Matrix Arrays [ true | false ], Print Timing Information [ true I false ], Undefined [ true I false ], 


\section{Comments}

The Initial Conditions Only option, when invoked (true), will force MSTS to read the "input" file, compute all secondary variables and fluxes (e.g., liquid saturation and Darcy velocities), and stop execution. This option is useful if the user wishes to confirm that the initial conditions are correct before proceeding with a lengthy simulation.

The Restart option permits a restart from a previous simulation. Whenever a simulation is completed, MSTS records a file named "restart," which can be read to begin a new simulation from the same conditions. To invoke this option, set the Restart option to "true" and ensure that a valid "restart" file is available in the same directory as the "input" file. The Restart from Time Zero option applies only to restarted simulations. If the Restart from Time Zero option is set to "true," then the simulation time begins at zero for a simulation where the initial conditions are taken from the "restart" file; if set to "false," then the time continues from the simulation time of the "restart" file.

The governing equations for conservation of component mass and thermal energy are considered coupled equations. Seven solution modes or combinations of the coupled conservation equations are available with MSTS. Refer to Section 2.4 for a description of the seven solution modes. Solution of the species conservation equation can be appended to any of the seven coupledequation solution modes.

\section{Examples}

In this example, a simulation is started from initial conditions specified in the "input" file (not a "restart") and is solved using the banded matrix solution scheme. Only the water conservation equation will be solved for a simulated time of 2 hours beginning with an initial time step of 0.01 hour with a time-step acceleration factor of 1.25 (i.e., each time step will be 1.25 times larger than the previous unless the convergence criterion is violated), up to a maximum time step of 0.10 hour. A screen echo of the progress of the simulation will be available, binary diffusion is not included, and fluid properties are fixed (usually this is chosen for isothermal problems only). The liquid phase will be simulated, but not the gas phase. The vapor-pressure-lowering feature is not activated either. No debugging output is requested (subroutine names, array output, or timing information). 


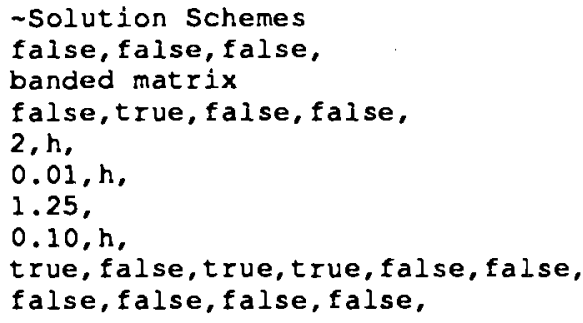

We can modify the same example for a second simulation in which thermal effects and the gas phase are modeled. In this case, we invoke two more equations (line 3), switch the Fixed Properties option to "false" because fluid properties will change with temperature, and switch the Binary Diffusion, Gas Phase, and Vapor Pressure Lowering options to "true," as follows:

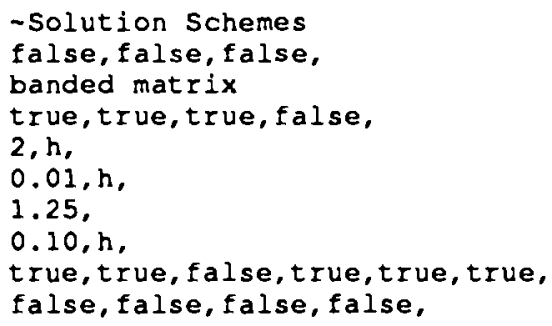




\subsubsection{Numerical Control Card}

\section{Function}

The Numerical Control card is used to control the iteration process, constrain the use of computer resources (limits on both total CPU usage and actual time), and specify the numerical convergence limits and averaging technique used for solution of each of the governing equations.

\section{$\underline{\text { Format }}$}

Line 0 " Numerical Control"

Line 1 Number of Newton-Raphson Iterations \{ integer \}, Residuals Convergence [ least squares I maximum residual ],

Line 2 CPU Execution Time Limit \{ real \}, Units $[s|\min | \mathrm{h} \mid$ day $|\mathrm{wk}| \mathrm{yr}]$, Clock Time Limit \{ real \}, Units $[s|\min | \mathrm{h} \mid$ day $|\mathrm{wk}| \mathrm{yr}]$,

Line 3 Thermal Energy Equation Convergence Criterion \{ real \}, Water Equation Convergence Criterion \{ real \}, Air Equation Convergence Criterion \{ real \},

Line 4 Thermal Energy Equation Mean [ harmonic I geometric I arithmetic I upwind ], Water Equation Mean [ harmonic I geometric l arithmetic I upwind ], Air Equation Mean [ harmonic I geometric I arithmetic I upwind ], Species Concentration Equation Mean [ harmonic I geometric I arithmetic I upwind ],

\section{Comments}

The CPU execution time and clock time limits are provided to give a user the means to prevent a batch-process run of the MSTS code from exceeding queue limits in a mainframe computer. For example, if the host computer queue limits a batch submission to $4 \mathrm{CPU}$-hours, the CPU execution time limit can be set to 3.9 hours to prevent an interruption. MSTS will execute for 3.9 hours and write the requested output files for the last time step completed at that time, including a "restart" file. If additional time is required to finish the simulation, the "restart" file can then be used to continue. The clock time limit works in the same manner.

Convergence limits need be specified only for those coupled governing equations that are to be solved, as indicated by options in the Solution Schemes card. 


\section{Examples}

For this example, we choose to specify eight Newton-Raphson iterations using the Maximum Residual Convergence option. The simulation will be stopped and a "restart" file written for possible later use if the simulation consumes more than 1 hour of CPU time or 5 hours of wallclock time. The Convergence Criterion field for each of the three coupled governing equations is $1.0 \times 10^{-6}$, and all internodal properties will be computed using a harmonic mean.

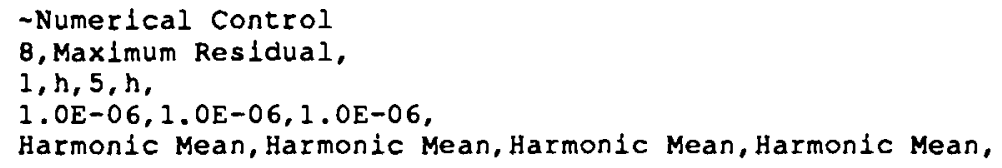

The first example could be modified to allow 10 Newton-Raphson iterations per time step, extend the CPU execution limit to 6 hours, extend the clock limit to 1 day, and require a geometric mean for solution of the water conservation equation, as follows:

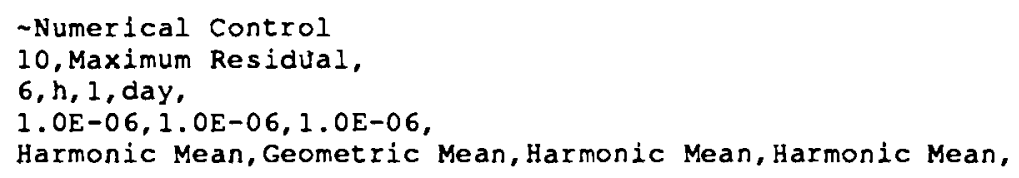




\subsection{Computational Domain}

The computational domain is defined using the Grid Geometry, Inactive Nodes, and Rock or Soil Types cards. The first of these, Grid Geometry card, is used to specify the coordinate system (Cartesian and cylindrical are available), the total physical length of the model domain in each dimension, the number of nodes in each dimension, the elevation datum, and the location of each node. Often the rectangular grid required by the finite-difference method used in MSTS does not provide the flexibility needed to describe complex geometries; the Inactive Nodes card provides a way to "blank out" noncomputational nodes, which MSTS will not treat as a part of the porous media continuum in a simulation. The inactive nodes feature is useful, for example, when modeling a geometry in which several nodes in the corner of a model domain are in the atmosphere; impervious regions also could be modeled as inactive nodes. The Rock or Soil Types card is used to assign ranges of nodes to a rock or soil type. The rock or soil type is commonly identified by a name, such as "Ringold Formation" or "Willamette Silt Loam." All physical properties of these rock or soil types are defined in subsequent input cards and assigned to the type by the identifying name. 


\subsubsection{Grid Geometry Card}

Function

The Grid Geometry card is used to select the problem coordinate system (Cartesian or cylindrical), specify the number of nodes, specify the physical extent of the problem domain, and indicate the physical location of all nodes in the problem domain.

\section{Format}

Line 0

"—Grid Geometry"

Line 1 Coordinate System [ Cartesian I cylindrical ],

Line 2 Number of Grid Nodes in the $x$ - or $r$-Direction \{ integer \}, Number of Grid Nodes in the $y$ - or $\theta$-Direction \{ integer \}, Number of Grid Nodes in the $z$-Direction [integer \},

Line $3 x$-or $r$-Direction Physical Domain Length $\{$ real $\}$, Units $[\mathrm{m}|\mathrm{ft}| \mathrm{cm}]$, $y$ - or $\theta$-Direction Physical Domain Length or Arc \{real \},

Units $[\mathrm{m} / \mathrm{ft} \mid \mathrm{cm} /$ degrees ], $z$-Direction Physical Domain Length [ real \}, Units $[\mathrm{m}|\mathrm{ft}| \mathrm{cm}]$, z-Direction Datum $z^{*}\{$ real $\}$, Units $[\mathrm{m}|\mathrm{ft}| \mathrm{cm}]$, $z$-Direction Grid Bottom Elevation from Datum \{ real \}, Units $[\mathrm{m} \mid \mathrm{ft} / \mathrm{cm}]$,

Line 4 Inner Radius Length $r_{0}\{$ real (cylindrical coordinates; enter zero for Cartesian) \}, Units $[\mathrm{m} / \mathrm{ft} \mid \mathrm{cm}]$,

Line $5 \quad$ I \{ integer \} "line(s) of grid geometry input follows."

Line $\mathrm{i} \quad$ "Node " $\mathrm{i}\{$ integer \}, $x$ - or $r$-Direction Node Position \{ real \}, Units $[\mathrm{m}|\mathrm{ft}| \mathrm{cm}]$,

Next Line $\quad J\{$ integer \} "line(s) of grid geometry input follows."

Line $\mathrm{j} \quad$ "Node " $\mathrm{j}\{$ integer \}, $y$ - or $\theta$-Direction Node Position ( real ), Units $[\mathrm{m}|\mathrm{ft}| \mathrm{cm} \mid$ degrees ],

Next Line $\quad \mathrm{K}\{$ integer $\}$ " line(s) of grid geometry input follows."

Line $\mathbf{k} \quad$ "Node " $\mathbf{k}$ \{ integer \}, $z$-Direction Node Position \{ real \}, Units $[\mathrm{m} / \mathrm{ft} / \mathrm{cm}]$, 


\section{Comments}

The Cartesian coordinate system is used to represent any length, area, or volume in rectangular grid volumes. Cartesian coordinates always imply the horizontal plane is represented by $x$ - and $y$-axes, and the vertical plane (coinciding with the gravity vector) by the $z$-axis. The cylindrical coordinate system can be used to represent a radial, symmetric problem only, centered on a point. The horizontal plane in this case is represented by the radius $r$ and angle $\theta$. The vertical plane is represented by the $z$-axis, just as for Cartesian coordinates. Any portion of an arc greater than 0 degrees but less than 360 degrees may be represented with cylindrical coordinates, though the symmetry requirement would imply that a smaller, representative arc would be sufficient for most radial problems.

Node positions are referenced from the west, south, or bottom boundaries for the $x-, y-$, and $z$-coordinate directions, respectively, for Cartesian coordinate systems. For cylindrical coordinate systems, node positions are referenced from the west, south, or bottom boundaries for the $r-, \theta, z-$ coordinate directions, respectively. Surfaces between nodes are located at the midpoint between two adjacent node positions. Node volumes are defined by their bounding surfaces. To illustrate, a onedimensional probiem $3 \mathrm{~m}$ in length could be represented by three uniformly distributed nodes in the appropriate dimension, located at $0.5,1.5$, and $2.5 \mathrm{~m}$, as shown in Figure 6.1. For a nonuniform distribution of nodes, a node is not necessarily centered in the grid cell it represents. Figure 6.2 illustrates the same one-dimensional, three-cell problem of 3-m length represented by nonuniform node spacing at 1.0, 1.5, and $2.5 \mathrm{~m}$. The first grid cell, which is the length represented by node 1 , extends from the left domain boundary $(x=0)$ to the midpoint between the first and second nodes $(x=1.25 \mathrm{~m})$.

In MSTS, the physical position of every node must be a positive number. MSTS grid geometries should generally arrange a grid such that the lower boundary in each dimension is at position zero. The vertical dimension can be offset to a different reference by means of the zDirection Grid Bottom Elevation from Datum field value. For example, if the bottom of a model domain is at elevation $700 \mathrm{~m}$ above mean sea level and the top is at $1000 \mathrm{~m}$, distribute $z$-direction nodes from zero to $300 \mathrm{~m}$ in the $\mathrm{K}$ dimension and assign a value of $700 \mathrm{~m}$ to the $\mathrm{z}$-Direction Grid Bottom Elevation from Datum field. 


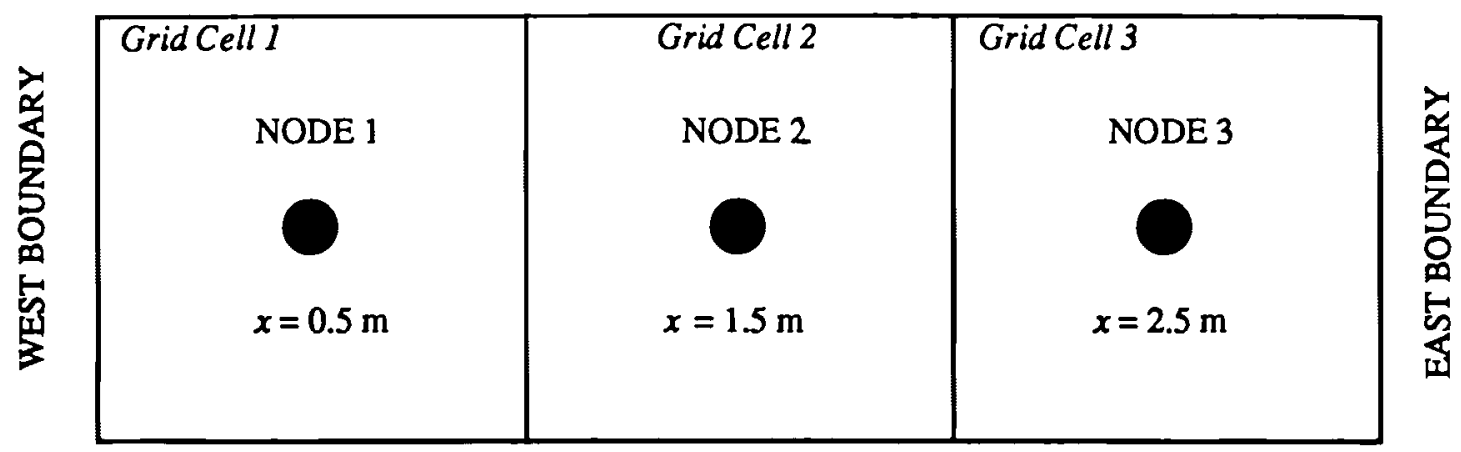

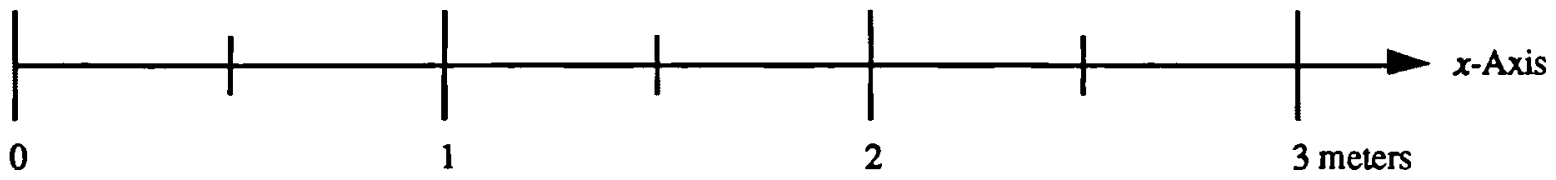

Figure 6.1. Example Grid Nodes and Associated Grid Cells for Uniform Node Distribution

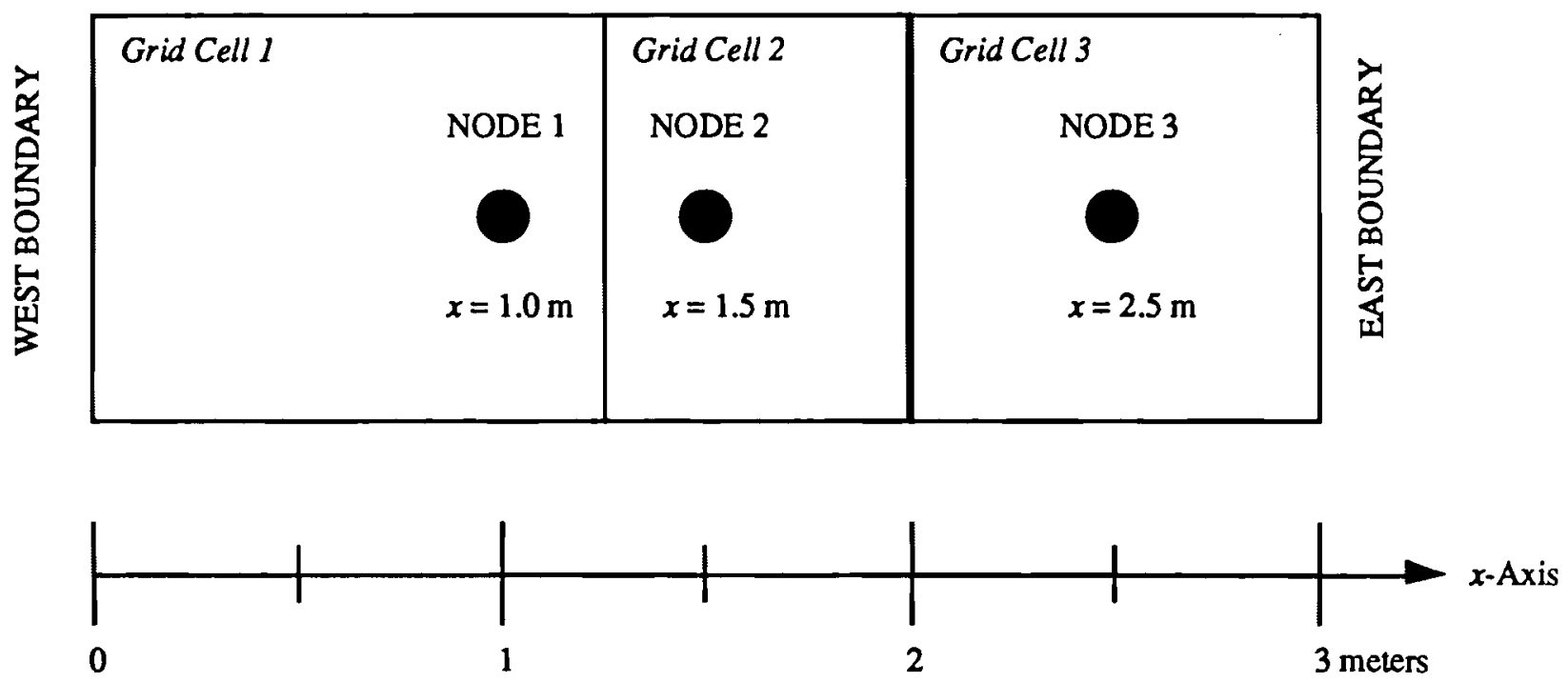

Figure 6.2. Example Grid Nodes and Associated Grid Cells for Nonuniform Node Distribution

\section{Examples}

The first example shows how the problem illustrated in Figure 6.1 would be defined using the Grid Geometry card. This problem is a single-dimension (in the $x$-direction) domain $3 \mathrm{~m}$ in length to be represented by three nodes. The $y$ - and $z$-direction nodes are given a single node each to indicate the one-dimensional aspect of the problem. 


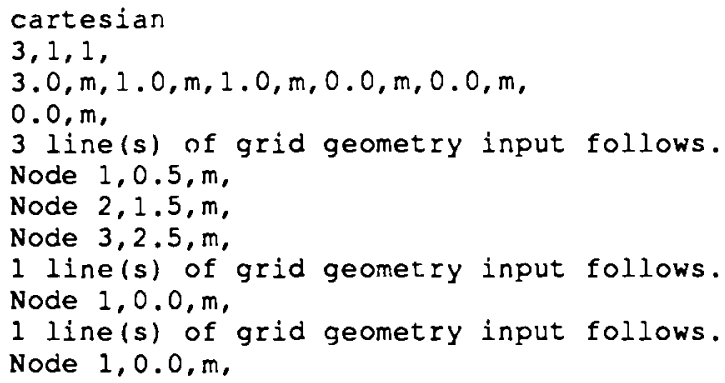

A vertical domain is similar, except the $z$-axis is the multiple node direction. The next example illustrates use of the Grid Geometry card for a one-dimensional (vertical), five-node problem that models a physical length of $10.0 \mathrm{~cm}$. The top of the domain is taken as elevation zero, so the bottom of the domain is at elevation $-10.0 \mathrm{~cm}$.

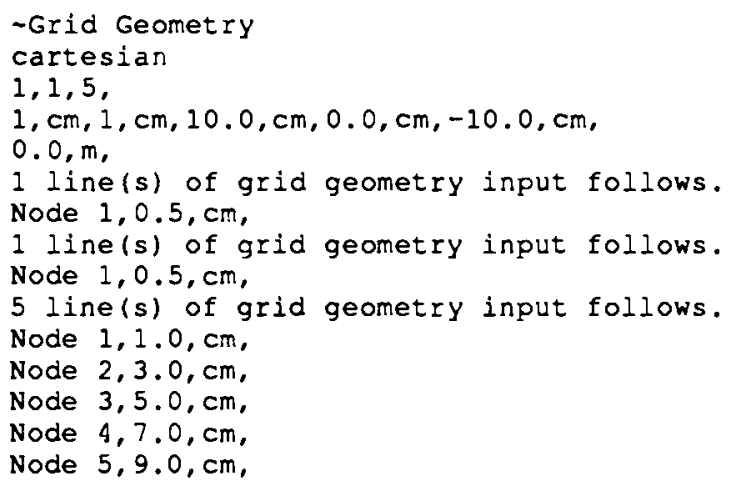

For a radially symmetric, 10-m-tall, 1 -m-radius problem centered on a borehole with inner radius $10 \mathrm{~cm}$ and represented by a 10-degree arc, the Grid Geometry card would look like:

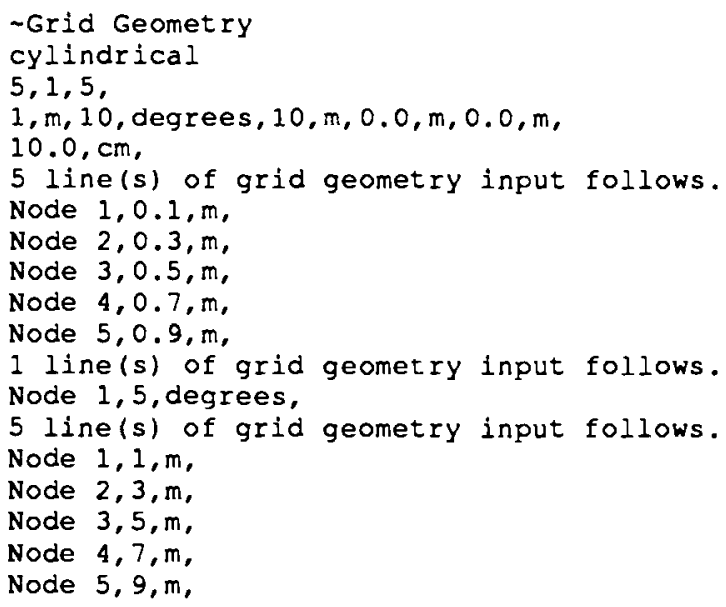




\subsubsection{Inactive Nodes Card}

\section{Eunction}

The Inactive Nodes card is used to designate inactive, or noncomputational, nodes in the problem domain. Inactive nodes may represent any portions of the model domain that are to be excluded from the porous media continuum to be simulated. Examples might include nodes representing the atmosphere or impervious rock. Inactive nodes can also be used to define internal boundary conditions. A surface between an inactive and an active node can function as a boundary surface for the active node. Normal boundary conditions may be applied to these internal surfaces.

Format

Line $0 \quad$ " Inactive Nodes"

Line $1 \quad \mathrm{~N}$ \{ integer \} " line(s) of inactive nodes input follows."

Line n I Start \{ integer \}, I End \{ integer \}, J Start \{ integer \}, J End ( integer ), K Start ( integer \}, $\mathrm{K}$ End \{ integer \},

\section{Comments}

The restriction to rectangular elements imposed by the finite-difference method used in the MSTS code makes descriptions of irregular geometries difficult. The inactive nodes feature of MSTS gives some flexibility in this regard by allowing the user to specify nodes at which the governing equations will not be solved and which can be assigned boundary conditions. Although grid cells are still restricted to rectangular elements, the model domain is not. Examples of appropriate uses for inactive nodes include representation of the atmosphere for a sloping surface at the upper part of a model, representation of a nonhorizontal saturated zone in a unsaturated model domain, and representation of impervious zones within a model domain.

\section{Examples}

This example makes two groups of nodes inactive; one group will represent the atmosphere in the upper left-hand portion of a two-dimensional ( 10 by 1 by 5 node) domain, and the other group will represent an impervious region in the lower left-hand portion. 
- Inactive Nodes

2 line(s) of inactive nodes input follows.

$8,10,1,1,5,5$,

$1,3,1,1,1,2$,

The following example declares that there are no inactive nodes in the model domain:

- Inactive Nodes

O line(s) of inactive nodes input follows. 


\subsubsection{Rock or Soil Types Card}

\section{Function}

The Rock or Soil Types card is used to group computational node(s) with identical physical properties into unique rock or soil types.

\section{Format}

Line $0 \quad$ "Rock or Soil Types"

Line 1 Characters [ integer \} "character(s) of rock or soil types notes follows."

Line 2+ Rock Notes \{ alpha-numeric (strictly 80 characters per line) \}

Next Line M ( integer ] " line(s) of rock or soil types input follows."

Line $\mathrm{m} \quad$ Rock or Soil Type (alpha ( 80 characters maximum ) \},
I Start \{ integer \},
I End ( integer \},
J Start \{ integer \},
$J$ End \{ integer \},
$\mathrm{K}$ Start ( integer \},
$\mathrm{K}$ End ( integer \},

\section{Comments}

The Rock Notes field is used to include references or sources of information that help in documenting where the user obtained the properties used to represent rock or soil types.

The Rock or Soil Types card is used to specify properties for similar groups of nodes, often termed zones, units, or layers. Usually groupings follow hydrogeologically similar soils or rock formations. The Rock or Soil Type field is a name that identifies the grouping to which properties are assigned, e.g., "Tiva Canyon Welded" or "Yolo Light Clay." The next six numbers bound the rectangular block of nodes that are assigned to that rock or soil type. Multiple lines can be used to assign different groups of nodes to the same rock type (see the following examples).

\section{Examples}

In the first example, a one-dimensional vertical column of Yolo light clay is specified as the only soil type in the simulation and all 100 nodes of the problem are assigned to this type: 
$\sim$ Rock or soil Types

52 character(s) of rock or soil types notes follows.

Yolo light clay was first described by Moore (1939).

1 line(s) of rock or soil types input follows.

Yolo Light Clay, 1,1,1,1,1,100,

Often it is necessary to use multiple lines to assign all of the nodes belonging to one rock or soil type. Here the first example is repeated with a middle layer of 21 nodes assigned to a different rock type (generic sand):

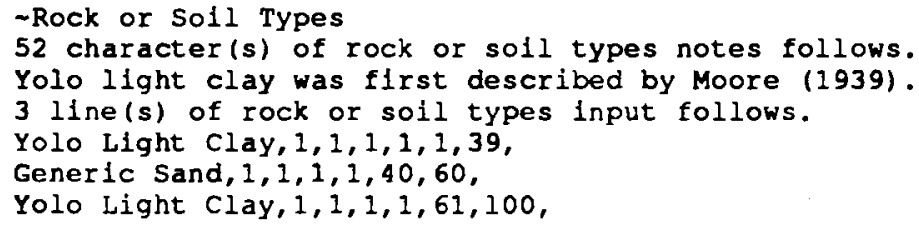




\subsection{Physical Properties}

Physical properties include mechanical properties (porosity and tortuosity), hydraulic properties (permeability or conductivity, and storativity), thermal properties (thermal conductivity, density, and specific heat), species properties (species name, molecular diffusion, liquid-gas partition coefficient, solid-liquid partition coefficient, and species half-life), soil characteristics (moisture-retention relations), liquid relative permeability, and gas relative permeability. All of the physical characteristics of a rock or soil type used by the MSTS code are entered using these seven physical properties input cards. In each card, the physical properties are assigned to a rock or soil name that must match precisely (except that MSTS input is case-insensitive) one of the names entered with the Rock or Soil Types card. 


\subsubsection{Mechanical Properties Card}

Function

The Mechanical Properties card is used to assign values of porosity and tortuosity to rock or soil types identified with the Rock or Soil Types card.

\section{Format}

Line $0 \quad$ " Mechanical Properties"

Line $1 \quad \mathrm{~N}$ ( integer \} " line(s) of mechanical properties input follows."

Line n Rock or Soil Type \{ alpha ( 80 characters maximum ) \}, Matrix Total Porosity $n_{T}$ or $n_{T}$ ( real \}, Matrix Diffusive Porosity $n_{D}$ or $n_{D_{m}}\{$ real $\}$, Matrix Effective Porosity $n_{E}$ or $n_{E_{m}}\{$ real $\}$, Fracture Total Porosity $n_{T}\{$ real $\}$, Fracture Diffusive Porosity $n_{D}\{$ real $\}$, Fracture Effective Porosity $n_{E}\{$ real $\}$, Tortuosity variability option [ constant I saturation dependent ], Liquid Tortuosity $\tau_{\ell}$ (not required for saturation-dependent option) $\{$ real $\}$, Gas Tortuosity $\tau_{g}$ (not required for saturation-dependent option) \{ real \},

\section{Comments}

Mechanical properties are assigned by rock or soil type, which is input using the Rock or Soil Types card. The Rock or Soil Type field must contain a name that exactly matches one contained on the Rock or Soil Types card.

The (matrix) total, effective, and diffusive porosities must be specified for all rock or soil types named. The fracture porosities must be named only for rock or soil types for which the dualporosity (equivalent-continuum) model is to be used.

Tortuosities can be specified under two options: "Constant" and "Saturation Dependent". If the Constant option is chosen, then a liquid-phase and/or gas-phase tortuosity must be specified. Zero values of tortuosity eliminate binary diffusion of gas-phase components and eliminate molecular diffusion for species transport. If the Saturation Dependent option is chosen, the liquidand gas-phase tortuosities are computed from a model proposed by Millington and Quirk (1959), where the tortuosities are computed as a function of the porosity and phase saturation. Refer to Section 3.8 of the MSTS Theory Manual (White and Nichols 1992) for a model description. 


\section{Examples}

In this example a single soil type, Yolo light clay, is assigned a total porosity of 0.495 , an effective porosity of 0.371 , and a diffusive porosity of 0.371 . Only matrix flow is applicable to this soil type, so nothing is put between the three commas normally reserved for fracture porosities.

The blank fields of tortuosity will result in constant tortuosities with zero value for both liquid and gas phases (i.e., no binary diffusion and no species diffusion transport).

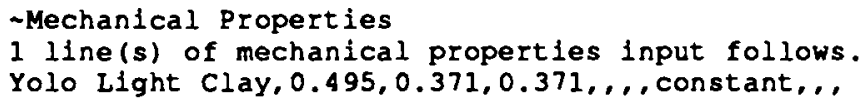

If the dual-porosity, equivalent-continuum approach is used for a rock type, then six porosity values are required. In this case, we specify two rock types, one that is fractured (Topopah Springs) and one that is not (Calico Hills). We also specify constant liquid and gas tortuosities:

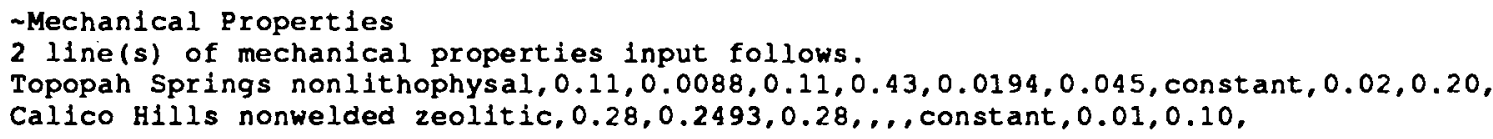




\subsubsection{Hydraulic Properties Card}

\section{Function}

The Hydraulic Properties card is used to assign values of intrinsic permeability or hydraulic conductivity to rock or soil types identified with the Rock or Soil Types card.

\section{Format}

Line 0

" Hydraulic Properties"

Line $1 \quad \mathrm{~N}$ \{ integer \} " line(s) of hydraulic properties input follows."

Line n Rock or Soil Type \{ alpha ( 80 characters maximum ) \}, $x$ - or $r$-Direction Matrix Intrinsic Permeability or Hydraulic Conductivity \{ real \}, $y$ - or $\theta$-Direction Matrix Intrinsic Permeability or Hydraulic Conductivity \{ real \}, z-Direction Matrix Intrinsic Permeability or Hydraulic Conductivity [ real \}, Units $\left[\mathrm{m}^{\wedge} 2\left|\mathrm{ft}^{\wedge} 2\right| \mathrm{cm}^{\wedge} 2|\mathrm{hc}: \mathrm{m} / \mathrm{s}| \mathrm{hc}: \mathrm{ft} / \mathrm{s}|\mathrm{hc}: \mathrm{cm} / \mathrm{s}| \mathrm{hc}: \mathrm{cm} /\right.$ day $]$, $x$ - or $r$-Direction Fracture Intrinsic Permeability or Hydraulic Conductivity \{ real \}, $y$ - or $\theta$-Direction Fracture Intrinsic Permeability or Hydraulic Conductivity \{ real \}, $z$-Direction Fracture Intrinsic Permeability or Hydraulic Conductivity \{ real \}, Units [ $\mathrm{m}^{\wedge} 2\left|\mathrm{ft} \mathrm{f}^{\wedge}\right| \mathrm{cm}^{\wedge} 2|\mathrm{hc}: \mathrm{m} / \mathrm{s}| \mathrm{hc}: \mathrm{ft} / \mathrm{s}|\mathrm{hc}: \mathrm{cm} / \mathrm{s}| \mathrm{hc}: \mathrm{cm} /$ day $]$, Coefficient of Specific Storage $S_{s}\{$ real $\}$, Units $[1 / \mathrm{m}|1 / \mathrm{ft}| 1 / \mathrm{cm}]$,

\section{Comments}

Hydraulic properties are assigned by rock or soil type, which is input using the Rock or Soil Types card. The Rock or Soil Type field must contain a name that exactly matches one contained on the Rock or Soil Types card.

Enter values for fracture permeability (conductivity) only if you wish to invoke the equivalent-continuum, dual-porosity model (see Section 7.8 for details). Otherwise, enter only the matrix values and leave no characters between the commas for fracture values.

If only the water conservation equation is to be solved and it is preferable to enter hydraulic conductivity values rather than intrinsic permeability, precede the units with "hc:" to alert the MSTS code to this. For example, an isotropic hydraulic conductivity of $3.5 \mathrm{~cm} / \mathrm{s}$ would be entered as:

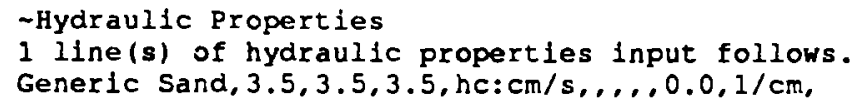


Hydraulic conductivity is converted to intrinsic permeability and storativity is converted to porous media compressibility internally in MSTS by assuming a reference temperature of $20^{\circ} \mathrm{C}$. Notice that no fracture values were included in this example, so this material would be treated as a simple porous medium.

\section{Examples}

The following example provides input for a single rock or soil type, Yolo light clay, with an isotropic hydraulic conductivity of $1.23 \times 10^{-7} \mathrm{~m} / \mathrm{s}$ and a storativity of $0.1241 / \mathrm{m}$.

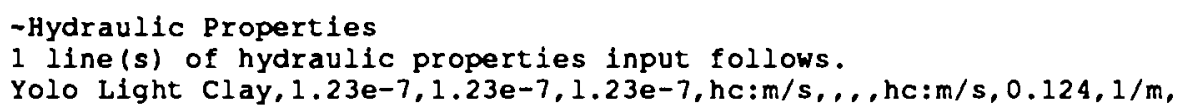

If we entered the same properties using intrinsic permeabilities, the input card would look like this:

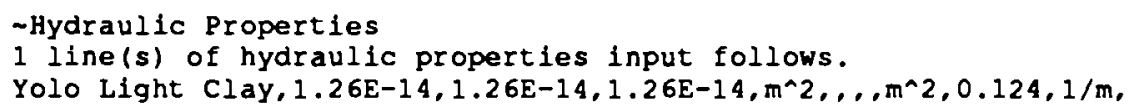

The next example gives the parameters for a simulation that will use the equivalentcontinuum, dual-porosity approach for one of two rock or soil types. The first layer is the Calico Hills nonwelded vitric, which has a saturated, isotropic hydraulic conductivity of $8.0 \times 10^{-11} \mathrm{~m} / \mathrm{s}$ and is not fractured. The second layer is the Topopah Springs welded nonlithophysal with a saturated, isotropic matrix hydraulic conductivity of $2.00 \times 10^{-11} \mathrm{~m} / \mathrm{s}$ and a saturated, isotropic fracture hydraulic conductivity of $8.3 \times 10^{-5} \mathrm{~m} / \mathrm{s}$.

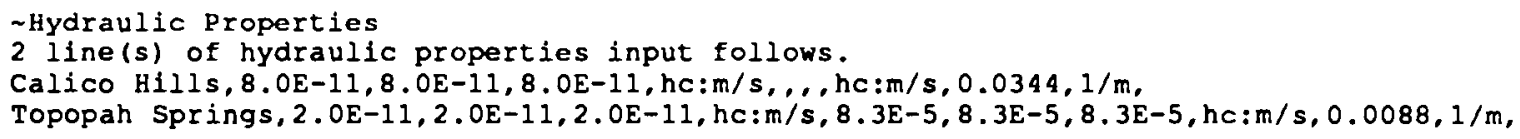




\subsubsection{Thermal Properties Card}

\section{Function}

The Thermal Properties card is used to assign values of thermal conductivity, density, and specific heat to rock or soil types identified with the Rock or Soil Types card.

\section{Format}

Line $0 \quad$ "Thermal Properties"

Line $1 \quad \mathrm{~N}$ [ integer ] " line(s) of thermal properties input follows."

Line n Rock or Soil Type \{ alpha ( 80 characters maximum ) \}, Effective Thermal Conductivity Model [ dependent I independent I somerton ] $x$ - or $r$-Direction Unsaturated Thermal Conductivity \{ real \}, $y$ - or $\theta$-Direction Unsaturated Thermal Conductivity \{ real \}, $z$-Direction Unsaturated Thermal Conductivity \{ real \}, $x$ - or $r$-Direction Saturated Thermal Conductivity \{ real \}, $y$ - or $\theta$-Direction Saturated Thermal Conductivity \{ real \}, $z$-Direction Saturated Thermal Conductivity \{ real \}, Units [ $\mathrm{W} / \mathrm{m} \mathrm{K}|\mathrm{Btu} / \mathrm{h} \mathrm{ft} \mathrm{F} / \mathrm{cal} / \mathrm{s} \mathrm{cm} \mathrm{K}| \mathrm{Btu}$ in $/ \mathrm{h} \mathrm{ft} \wedge 2 \mathrm{~F}$ ], Rock or Soil Density $\rho_{s}$ ( real ], Units [ $\left.\mathrm{kg} / \mathrm{m}^{\wedge} 3\left|\mathrm{lbm} / \mathrm{ft}^{\wedge} 3\right| \mathrm{gm} / \mathrm{cm}^{\wedge} 3\right]$, Rock or Soil Specific Heat $c_{s}\{$ real \}, Units [ J/kg K I Btu/lbm F I cal/gm K ],

\section{Comments}

Thermal properties are assigned by rock or soil type, which is input using the Rock or Soil Types card. The Rock or Soil Type field must contain a name that exactly matches one contained on the Rock or Soil Types card. The equivalent thermal conductivity of a partially saturated porous medium can be computed using three different models in MSTS. These models are described in Section 3.9 of the MSTS Theory Manual (White and Nichols 1992). The model choice is specified with the second option as "Independent," "Dependent," or "Somerton." The Dependent option combines a temperature-dependent liquid conductivity with an unsaturated solid thermal conductivity as a function of moisture content. The Independent option scales the saturated and unsaturated thermal conductivities with the liquid saturation. The Somerton option scales the saturated and unsaturated thermal conductivities with the square root of the liquid saturation. 


\section{Examples}

The first example illustrates how to enter no thermal energy physical parameters at all for an isothermal simulation:

- Thermal Properties

o line(s) of thermal properties input follows.

A problem with one rock or soil type of an isotropic material that has a thermal conductivity of $2.10 \mathrm{~W} / \mathrm{m} \mathrm{K}$, a density of $2650 \mathrm{~kg} / \mathrm{m}^{3}$, and a specific heat of $1000 \mathrm{~J} / \mathrm{kg} \mathrm{K}$ would be entered as this example shows:

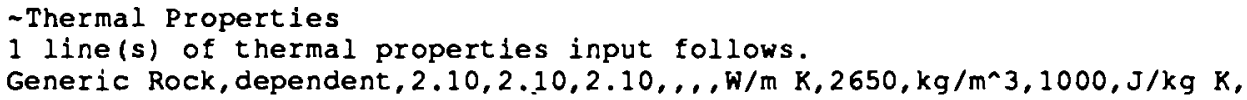

If the same rock type were anisotropic, having a thermal conductivity twice as large in the vertical dimension as in the horizontal dimensions, the above example would be modified to appear as

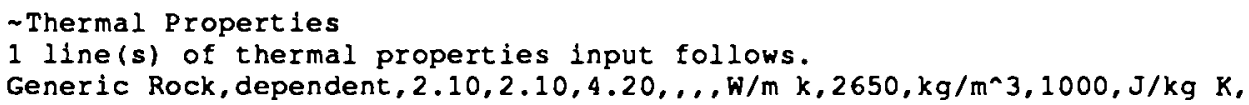

Finally, a three-rock domain could be defined with the following Thermal Properties card:

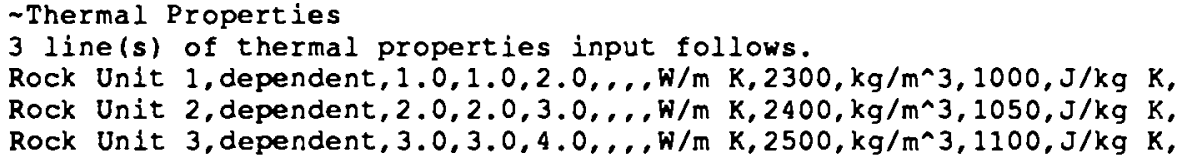




\subsubsection{Species Properties Card}

\section{Function}

The Species Properties card is used to assign a species name and associated molecular diffusion coefficients, partition coefficients, and radioactive half-life to each rock or soil type identified with the Rock or Soil Types card. The current version of MSTS can simulate only one species at a time, but the species properties need to be identified with respect to each rock or soil type.

\section{Format}

Line 0

$$
\text { "-Species Properties" }
$$

Line $1 \quad \mathrm{~N}$ \{ integer \} "line(s) of species properties input follows."

Line n Rock or Soil Type \{ alpha ( 80 characters maximum ) \}, Species Name \{ alpha ( 80 characters maximum ) \}, Liquid-Phase Molecular Diffusion Coefficient $D_{d_{k}}$ at $20^{\circ} \mathrm{C}$ and $1 \mathrm{~atm}$ \{ real \}, Units $\left[\mathrm{m}^{\wedge} 2 / \mathrm{s}\left|\mathrm{ft}^{\wedge} 2 / \mathrm{s}\right| \mathrm{cm}^{\wedge} 2 / \mathrm{s}\right]$, Gas-Phase Molecular Diffusion Coefficient $D_{d_{g}}$ at $20^{\circ} \mathrm{C}$ and $1 \mathrm{~atm}\{$ real \}, Units $\left[\mathrm{m}^{\wedge} 2 / \mathrm{s}\left|\mathrm{ft}^{\wedge} 2 / \mathrm{s}\right| \mathrm{cm}^{\wedge} 2 / \mathrm{s}\right.$ ], Liquid-Gas Partition Coefficient $K_{\ell g}\{$ real $\}$, Units [ $\mathrm{cm}^{\wedge} 3$ gas/gm liq. I liter gas/gm liq. ], Solid-Liquid Partition Coefficient $K_{d}\{$ real $\}$, Units [ gm liq./gm sol. ], Species Half-Life $t_{1 / 2}\{$ real \}, Units $[\mathrm{s}|\min | \mathrm{h} \mid$ day $|\mathrm{wk}| \mathrm{yr}]$,

\section{Comments}

Species properties are indexed by rock or soil types because the molecular diffusion coefficients and phase partition coefficients may be dependent on the local geochemical environment. The Rock or Soil Type field must contain a name that exactly matches one contained on the Rock or Soil Types card. The liquid-gas partition coefficient equals the ratio of species concentration in the liquid phase to that in the gas phase. Similarly, the solid-liquid partition coefficient equals the ratio of the species concentration adsorbed on the solid phase to that dissolved in the liquid phase.

\section{Examples}

If the species mass conservation equation is not solved, the Species Properties card is entered 
A simple example of species transport for a contaminant transported in liquid phase only through a single rock domain without retardation is

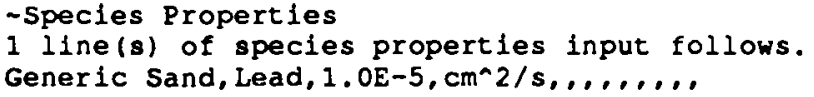

The following example illustrates the Species Properties card for ${ }^{14} \mathrm{C}$, a radioactive isotope of carbon with a half-life of 5730 years, in a five-rock domain of volcanic tuffs. Carbon- 14 is transported in both the liquid and the gas phases, so molecular diffusion and partition coefficients are all specified.

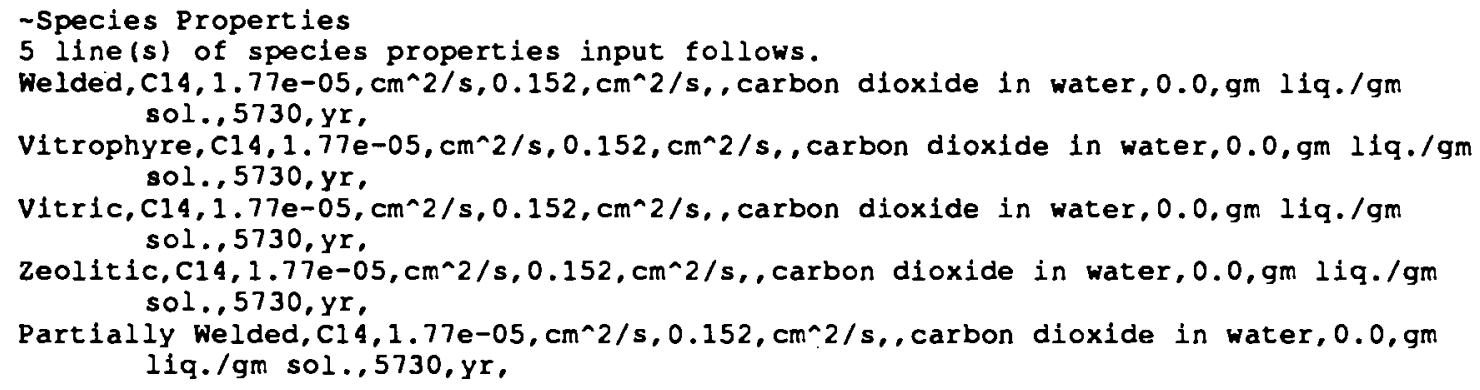

This example uses a special MSTS feature, "carbon dioxide in water," to describe the phase partition coefficients for the geochemical environment in the vadose zone at Yucca Mountain, Nevada. 


\subsubsection{Soil Characteristics Card}

\section{Function}

The Soil Characteristics card is used to specify the relationship between liquid-gas capillary pressure and liquid saturation for unsaturated conditions for each rock or soil type identified on the Rock or Soil Types card. Several functions are available to express the soil-moisture-retention characteristics: van Genuchten, Brooks and Corey, Haverkamp, Leverett, or dual porosity with van Genuchten, as well as tabular input (see Section 7.0 for a description of these functions). The maximum tension head for each rock or soil type is also specified on this card.

\section{Format}

Line $0 \quad$ "Soil Characteristics"

Line 1: $\quad \mathrm{N}\{$ integer $\}$ " line(s) of soil characteristics input follows." van Genuchten relation.

Line n Rock or Soil Type \{ alpha ( 80 characters maximum ) \}, "van Genuchten," van Genuchten $\alpha$ Parameter $\{$ real \}, Units [ $1 / \mathrm{cm} \mathrm{|} 1 / \mathrm{m} \mathrm{|} 1 / \mathrm{ft}]$, $\operatorname{van}$ Genuchten $n\{$ real (dimensionless ) \}, Maximum Tension Head \{ real \}, Units $[\mathrm{m} / \mathrm{ft} / \mathrm{cm}]$,

,

,

Brooks and Corey relation.

Line n Rock or Soil Type \{ alpha ( 80 characters maximum ) \}, "Brooks and Corey,"

Brooks and Corey $\psi^{*}$ parameter $\{$ real $\}$, Units $[\mathrm{cm}|\mathrm{m}| \mathrm{ft}]$, Brooks and Corey $n$ parameter $\{$ real $\}$, Maximum Tension Head \{ real \}, Units $[\mathrm{m}|\mathrm{ft}| \mathrm{cm}]$,

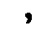


Haverkamp relation.

Line n Rock or Soil Type \{ alpha ( 80 characters maximum ) \}, "Haverkamp,"

Haverkamp $\alpha$ Parameter \{ real \},

Haverkamp $\beta$ \{ real (dimensionless ) \},

Maximum Tension Head \{ real \},

Units $[\mathrm{m} / \mathrm{ft} \mathrm{cm}$ ],

Leverett relation.

Line n Rock or Soil Type \{ alpha ( 80 characters maximum ) \}, "Leverett"

Residual Liquid Saturation $s_{\ell}\{$ real \},

Reference Capillary Pressure ( real ( Pa ) \},

Maximum Tension Head \{ real \},

Units $[\mathrm{m} / \mathrm{ft} \mid \mathrm{cm}]$,

Dual-Porosity model.

Line $\mathrm{n} \quad$ Rock or Soil Type \{ alpha ( 80 characters maximum ) \},

"Dual Porosity,"

van Genuchten $\alpha_{m}$ Parameter for Matrix \{real \},

Units [ $1 / \mathrm{cm}|1 / \mathrm{m}| 1 / \mathrm{ft}]$,

van Genuchten $n_{m}$ Parameter for Matrix \{ real \},

Maximum Tension Head \{ real \},

Units $[\mathrm{m}|\mathrm{ft}| \mathrm{cm}]$,

van Genuchten $\alpha_{f}$ Parameter for Fracture [ real \},

Units [ $1 / \mathrm{cm}|1 / \mathrm{m}| 1 / \mathrm{ft}]$

van Genuchten $n_{f}$ Parameter for Fracture \{ real \},

Tabular data.

Line $n \quad$ Rock or Soil Type ( alpha ( 80 characters maximum ) \}, "Tabular,"

"Table No. " $\mathrm{M}$ \{ integer \},

Tables for tabular data follow all functional input lines. Tables must be ordered sequentially according to their respective table numbers.

Line $1 \quad \mathrm{~N}$ \{ integer\} "line(s) of capillary tables input follows."

Line $\mathrm{n}$ Tension Head $\psi\{$ real $\}$,

Liquid Saturation $s_{\ell}\{$ real $\}$,

Tension Head Units [ $\mathrm{m}|\mathrm{ft}| \mathrm{cm}$ ], 


\section{Comments}

Soil characteristics are assigned by rock or soil type, which is input using the Rock or Soil Types card. The Rock or Soil Type field must contain a name that exactly matches one contained on the Rock or Soil Types card. Functional specifications of the soil-moisture-retention functions are preferred over tabular descriptions.

\section{Examples}

One method to specify the relationship between liquid pressure and liquid saturation is to use tabular input. For example, suppose the Haverkamp relations (Haverkamp et al. 1977) were not available in MSTS to define the soil-moisture-retention characteristics of a soil and we wished to use that function with the following parameters: $\alpha=200, \beta=1.5, A=100$, and $B=2$. We could compute several pairs of tension head-liquid saturation points from the Haverkamp function (see Section 7.3 for the equation) and enter these as tabular input in the MSTS "input" file. Points on the soil-moisture-retention curve between tabular values are derived in MSTS by linear interpolation; hence, the greater the accuracy required, the greater the number of points should be in the tabular input that defines a nonlinear relationship such as this one. The resulting tabular input might appear as follows:

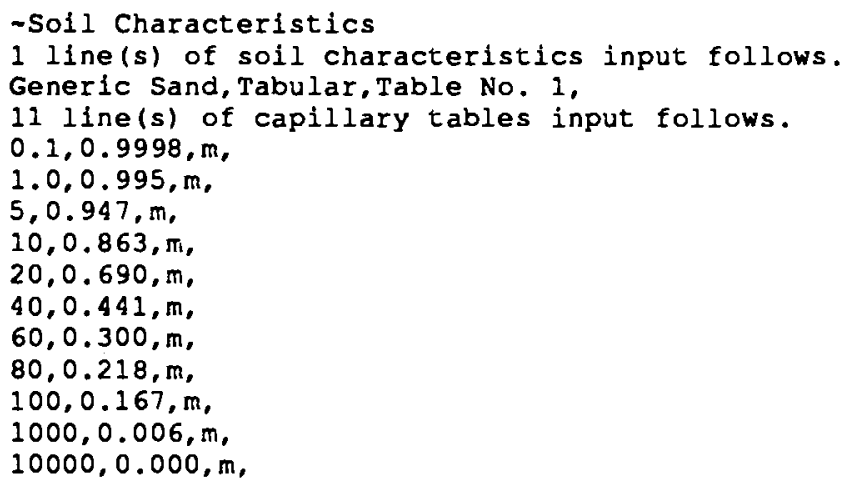

If the parameters are available, functional relationships are preferred to tabular input because data entry is easier, the problems with linear interpolations are avoided, and solutions will be faster than for tabular input. In the following example, the van Genuchten relations are used to define soil characteristics for nine "zones," or rock types, as follows: 


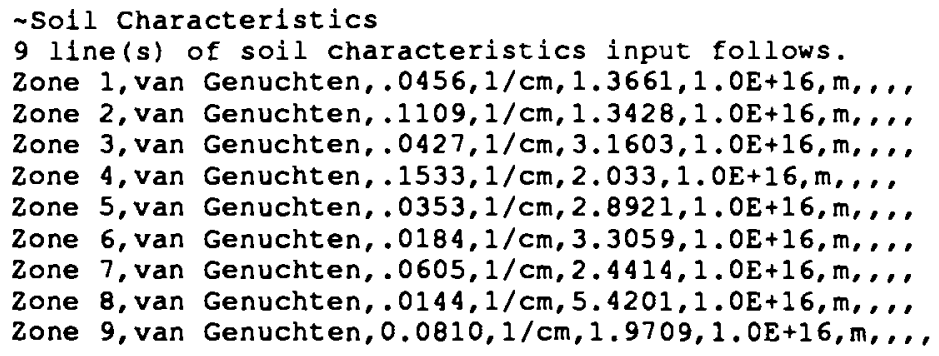

An example follows for the use of the Brooks and Corey function to define the soil-moistureretention curve of sandy clay:

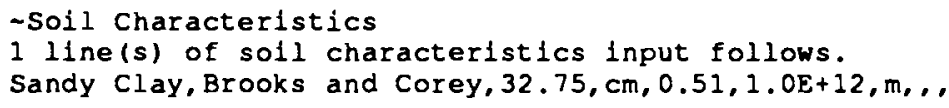

An example follows for the use of the Leverett function to define the soil-moisture-retention curve of sandy clay:

Soll characteristics
1 IIne(s) of soll characteristics input follows.
Zone 3 . Leverett, $0.05, .10132 .0,1.0 \mathrm{E}+15, \mathrm{~m}, \ldots$ 


\subsubsection{Liquid Relative Permeability Card}

\section{Function}

The Liquid Relative Permeability card is used to specify the liquid relative-permeability function for each rock or soil type identified on the Rock or Soil Types card. Several functions are available to express the relationship between liquid relative permeability and liquid saturation or between liquid relative permeability and capillary pressure head: Mualem pore distribution function integrated with the van Genuchten or Brooks and Corey soil-moisture-retention functions, Burdine pore distribution function integrated with the van Genuchten or Brooks and Corey soil-moistureretention functions, Haverkamp function, Corey function, Fatt and Klikoff function, Touma and Vauclin function, or a dual-porosity model. Alternatively, tabular input may be used to define the relationship between liquid relative permeability and liquid saturation or between liquid relative permeability and capillary pressure head. The minimum liquid relative permeability for each rock or soil type is specified with the Liquid Relative Permeability card also.

\section{Format}

Line $0 \quad$ "Liquid Relative Permeability Properties"

Line $1 \quad \mathrm{~N}$ \{ integer \} " line(s) of liquid relative permeability input follows."

Mualem function with van Genuchten soil-moisture-retention relation.

Line n Rock or Soil Type \{ alpha ( 80 characters maximum ) \}, "Mualem w/van Genuchten," van Genuchten $m$ Parameter \{real \}, Minimum Liquid Relative Permeability \{ real \},

Mualem function with Brooks and Corey soil-moisture-retention relation.

Line n Rock or Soil Type \{ alpha ( 80 characters maximum ) \}, "Mualem w/ Brooks \& Corey," Brooks and Corey $\beta$ Parameter \{ real \}, Minimum Liquid Relative Permeability \{ real \}, Burdine function with van Genuchten soil-moisture-retention relation.

Line n Rock or Soil Type \{ alpha ( 80 characters maximum ) \}, "Burdine w/van Genuchten," van Genuchten $m$ Parameter \{ real \}, Minimum Liquid Relative Permeability \{ real \}, 
Burdine function with Brooks and Corey soil-moisture-retention relation.

Line n Rock or Soil Type \{ alpha ( 80 characters maximum ) \}, "Burdine w/ Brooks \& Corey,"

Brooks and Corey $\beta$ Parameter \{ real \},

Minimum Liquid Relative Permeability \{ real \},

Haverkamp relation.

Line n Rock or Soil Type \{ alpha ( 80 characters maximum ) \},

"Haverkamp,"

Haverkamp $A$ Parameter \{ real \},

Haverkamp $B$ Parameter $\{$ real \},

Minimum Liquid Relative Permeability ( real \},

Corey relation.

Line n Rock or Soil Type \{ alpha ( 80 characters maximum ) \}, "Corey's Curves,"

Residual Liquid Saturation $s_{\ell}\{$ real \},

Entrapped Air Saturation $s_{\text {es }}\{$ real \},

Minimum Liquid Relative Permeability ( real \},

Fatt and Klikoff relation.

Line n Rock or Soil Type \{ alpha ( 80 characters maximum ) \}, "Fatt and Klikoff"

Residual Liquid Saturation $s_{\ell r}\{$ real $\}$,

Minimum Liquid Relative Permeability \{ real \},

Touma and Vauclin relation.

Line n Rock or Soil Type \{ alpha ( 80 characters maximum ) \},

"Touma and Vauclin"

Fitting Parameter $A_{\ell}\{$ real $\}$,

Fitting Parameter $B_{\ell}\{$ real $\}$,

Minimum Liquid Relative Permeability \{ real \},

Dual-porosity model.

Line n Rock or Soil Type \{ alpha ( 80 characters maximum ) \}, "Dual Porosity" van Genuchten $m_{m}$ Parameter for Matrix \{ real \},

Minimum Liquid Relative Permeability \{ real \}, van Genuchten $m_{f}$ Parameter for Fracture \{real \}, 
Tabular versus Liquid Saturation.

Line n Rock or Soil Type \{ alpha ( 80 characters maximum ) \}, "Tabular vs Liquid Saturation,"

"Table No. " M \{ integer \},

Tables for tabular data follow all functional input lines. Tables must be ordered sequentially according to their respective table numbers.

Line $1 \quad \mathrm{~N}\{$ integer $\}$ " line(s) of liquid permeability tables input follows."

Line n Liquid Saturation $s_{\ell}\{$ real $\}$,

Liquid Relative Permeability $k_{r \ell}$ \{ real \},

Tabular versus Tension Head.

Line n Rock or Soil Type ( alpha ( 80 characters maximum ) \},

"Tabular vs Tension Head,"

"Table No. " M [ integer \},

Tables for tabular data follow all functional input lines. Tables must be ordered sequentially according to their respective table numbers.

Line $1 \quad \mathrm{~N}$ ( integer \} " line(s) of liquid permeability tables input follows."

Line $n$ Tension Head $\psi\{$ real $\}$,

Liquid Relative Permeability $k_{r \ell}$ [ real \},

Tension Head Units $[\mathrm{m}|\mathrm{ft}| \mathrm{cm}$ ],

\section{Comments}

Liquid relative permeabilities are assigned by rock or soil type, which is input using the Rock or Soil Types card. The Rock or Soil Type field must contain a name that exactly matches one contained on the Rock or Soil Types card. Functional specifications of the liquid relativepermeability functions are preferred over tabular descriptions. For additional details on the various functional relationships (e.g., Mualem, Burdine) available with this card, refer to Section 7.0.

Examples

Tabular input is the most difficult to enter into MSTS input files, so the first two examples illustrate this type of specification for liquid relative permeability. The first example shows a tabular form of the liquid relative-permeability function proposed by Haverkamp et al. (1977), in the form of liquid relative permeability versus liquid saturation, as follows: 
- Liquid Relative Permeability Properties

1 line (s) of liquid relative permeability input follows.

Generic Sand, Tabular vs Liquid Saturation, Table No. 1,

11 line(s) of liquid permeability tables input follows.

$1.000,1.000$

$0.998,0.998$,

$0.947,0.800$

$0.863,0.500$

$0.691,0.200$

$0.442,0.059$

$0.301,0.027$,

$0.218,0.015$

$0.167,0.010$

$0.006,9.99 E-5$,

$0.000,1.00 \mathrm{E}-6$,

Alternatively, we can define the liquid relative permeability as a function of tension head, as follows:

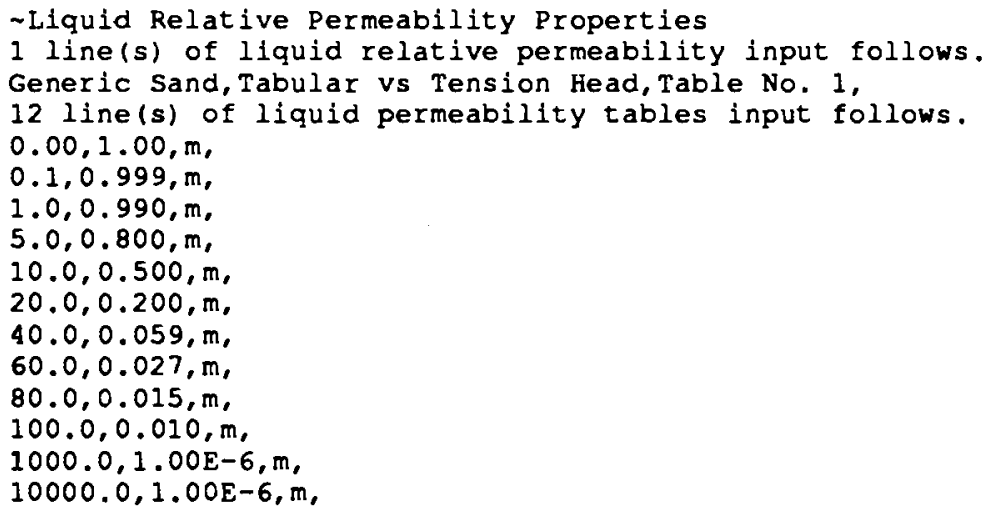

Notice in both tabular examples that the endpoints of the curves are established at zero capillary pressure $\left(s_{\ell}=1.0\right)$ and at the minimum relative conductivity for the problem $\left(1.0 \times 10^{-6} \mathrm{~m}\right.$ in this case); this practice serves to avoid undefined regions of these relationships.

Functional relations are easier to enter than tabular input. An example of the liquid relativepermeability function specified using the Corey function appears as follows:

- Liquid Relative Permeability Properties

1 line(s) of liquid relative permeability input follows.

Generic Sand, Corey's Curves, 0.30,0.05,1.0E-10,.

In this example, the minimum relative liquid permeability is $1.0 \times 10-10$, the parameter residual liquid saturation equals 0.30 , and the entrapped air at saturation equals 0.05 . See Section 7.0 for details on the parameters used in Corey's Curves and other relative-permeability functions.

The final example of liquid relative-permeability input is for a seven-rock-type problem where some of the rock types are fractured and use the dual-porosity model while others are not 
fractured and use Mualem functions with van Genuchten soil-moisture-retention relations.

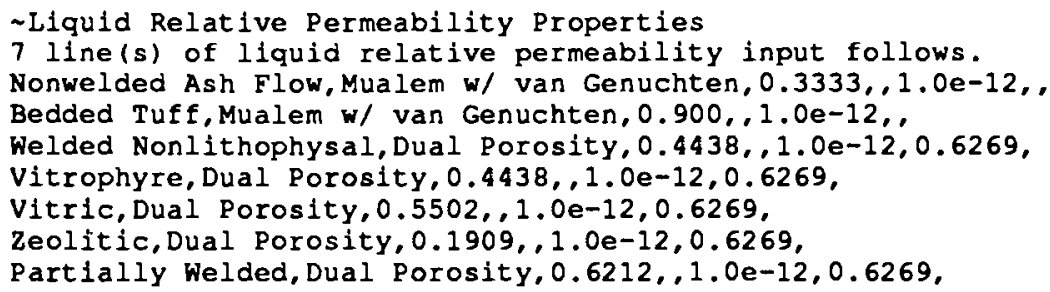




\subsubsection{Gas Relative Permeability Card}

\section{Eunction}

The Gas Relative Permeability card is used to specify the gas permeability function for each rock or soil type identified on the Rock or Soil Types card. Several functions are available to express the relationship between gas relative permeability and liquid saturation or between gas relative permeability and capillary pressure head: Corey function, Fatt and Klikoff function, Touma and Vauclin function, or a linear relationship with the liquid relative permeability of the same rock or soil type. Alternatively, tabular input may be used to define the relationship between gas relative permeability and liquid saturation or between gas relative permeability and capillary pressure head. The minimum gas relative permeability for each rock or soil type is also specified with the Gas Relative Permeability card.

\section{Format}

Line 0 "Gas Relative Permeability Properties"

Line $1 \quad \mathrm{~N}$ \{ integer \} " line(s) of gas relative permeability input follows."

\section{Corey relation.}

Line n Rock or Soil Type \{ alpha ( 80 characters maximum ) \}, "Corey's Curves,"

Residual Liquid Saturation $s_{\ell r}($ real $\}$,

Entrapped Air Saturation $s_{\ell s}$ ( real \},

Minimum Gas Relative Permeability \{ real \},

Fatt and Klikoff relation.

Line n Rock or Soil Type \{ alpha ( 80 characters maximum ) \},

"Fatt and Klikoff"

Residual Liquid Saturation $s_{\ell}\{$ real \},

Minimum Gas Relative Permeability \{ real \},

Touma and Vauclin relation.

Line n Rock or Soil Type \{ alpha ( 80 characters maximum ) \}, "Touma and Vauclin"

Fitting Parameter $A_{g}$ \{ real \},

Fitting Parameter $B_{g}\{$ real \},

Minimum Gas Relative Permeability \{ real \}, 
Linear with liquid relative permeability.

Line n Rock or Soil Type (alpha ( 80 characters maximum ) \}, "Linear w/Liq. Rel. Perm.,"

Tabular versus Liquid Saturation.

Line $n \quad$ Rock or Soil Type (alpha ( 80 characters maximum ) \},

"Tabular vs Liquid Saturation,"

"Table No." M \{ integer \},

Tables for tabular data follow all functional input lines. Tables must be ordered sequentially according to their respective table numbers.

Line $1 \quad \mathrm{~N}$ [ integer \} " line(s) of gas permeability tables input follows."

Line $n \quad$ Liquid Saturation $s_{\ell}\{$ real $\}$, Gas Relative Permeability $k_{r g}\{$ real $\}$,

Tabular versus Tension Head.

Line n Rock or Soil Type \{ alpha ( 80 characters maximum ) \},

"Tabular vs Tension Head,"

"Table No. " $M$ \{ integer \},

Tables for tabular data follow all functional input lines. Tables must be ordered sequentially according to their respective table numbers.

Line $1 \quad \mathrm{~N}$ \{ integer\} " line(s) of gas permeability tables input follows."

Line $n$ Tension Head $\psi$ ( real $\}$,

Gas Relative Permeability $k_{r g}$ \{ real \},

Tension Head Units $[\mathrm{m} \mid \mathrm{ft} / \mathrm{cm}$ ],

\section{Comments}

Gas relative permeabilities are assigned by rock or soil type, which is input using the Rock or Soil Types card. The Rock or Soil Type field must contain a name that exactly matches one contained on the Rock or Soil Types card. Functional specifications of the gas relative-permeability functions are preferred over tabular descriptions. For additional details on the various functional relationships (e.g., Corey, Fatt and Klikoff) available with this card, refer to Section 7.0.

\section{Examples}

As with liquid permeability relations, tabular input for gas permeability relations is the most complex type of entry. This example illustrates how to make gas relative permeability vary linearly with liquid saturation using tabular input for a single-rock-type problem: 
- Gas Relative Permeability Properties

1 line(s) of gas relative permeability input follows.

Generic Sand, Tabular vs Liquid Saturation, Table No. 1,

2 line(s) of gas permeability tables input follows.

$0.0,1.0$.

$1.0,1.0$.

Gas relative permeability can be expressed in terms of tension head (liquid-gas capillary pressure head), as well as liquid saturation. Of course, nonlinear relationships require more than two data points to adequately define a curve.

Another option for gas relative permeability is to relate it linearly to liquid relative permeability, as for this clay problem:

Gas Relative Permeability Properties
1 line(s) of gas relative permeability input follows.
Yolo Light Clay. Linear w/ Liq. Rel. Perm.,

The method used to define gas relative permeability can be different for different rock or soil types as well. Here, one rock type is described by a Fatt and Klikoff relation while a second is described by tabular input as a function of liquid saturation.

Gas Relative Permeability Properties

2 line(s) of gas relative permeability input follows.

Sample, Fatt and Klikoff,0.15, 1.0E-06,

Slick Rock, Tabular vs Liquid Saturation, Table No. 2,

3 line (s) of gas permeability tables input follows.

$0.0,0.4286$,

$0.15,0.4286$

$1.0,0.4286$, 


\subsection{Boundary Conditions}

Boundary conditions are specified through five distinct boundary condition cards: liquid, gas, vapor, thermal energy, and species. The Liquid Boundary Conditions card specifies boundary conditions for aqueous pressure, aqueous component mass fractions, aqueous density, and aqueous fluxes. The Gas Boundary Conditions card specifies boundary conditions for gas pressure, gas component mass fractions, gas density, and gas fluxes. The Vapor Boundary Condition card specifies boundary conditions only with respect to diffusion of components through the gas phase; the advection of components in the gas phase at boundary surfaces is specified through the gas boundary conditions. Having unique boundary conditions for the advection and diffusion transport mechanisms for gas components allows greater flexibility in creating problem definitions. For example, this approach allows the user to simulate boundaries that are impermeable to advective flow but permit gas component diffusion. The Energy Boundary Conditions card specifies boundary conditions for temperature, volumetric enthalpies for liquid and gas, and heat fluxes. The Species Boundary Conditions card specifies boundary conditions for species concentrations in the aqueous and gas phases and species boundary surface fluxes individually for the aqueous- and gasphase fluxes. The default boundary condition for every undeclared boundary surface is a zero-flux condition for liquid-phase mass, gas-phase mass, vapor diffusion, heat, and species mass. 


\subsubsection{Liquid Boundary Conditions Card}

\section{Eunction}

The Liquid Boundary Conditions card is used to specify boundary conditions for aqueous pressure, aqueous component mass fractions, aqueous density, and aqueous fluxes at the external boundaries of the problem domain and at the boundaries of inactive nodes. Four boundary condition types are available: Dirichlet, Neumann, saturated, and unit gradient.

Format

Line $0 \quad$ "Liquid Boundary Conditions"

Line $1 \quad \mathrm{~N}$ ( integer \} " line(s) of liquid boundary conditions input follows."

Dirichlet Boundary.

Line n Direction [ X-Direction / Y-Direction / Z-Direction ]:

Surface [ Top I Bottom I East I West I North I South ] " Surface,"

"Dirichlet @ Boundary,"

Liquid Pressure $P_{\ell}\{$ real $\}$,

Units [ $\mathrm{Pa}$ (absolute) | Psi (absolute) | Atm (absolute) | Bar (absolute) ],

Liquid Density $\rho_{\ell}\{\mathrm{real}\}$,

Units [ $\mathrm{kg} / \mathrm{m}^{\wedge} 3|\mathrm{lbm} / \mathrm{ft} \wedge 3| \mathrm{gm} / \mathrm{cm}^{\wedge} 3$ ],

Air Mass Fraction $x q\{$ real $\}$,

I Start \{ integer \},

I End ( integer \},

J Start $\{$ integer \},

$\mathrm{J}$ End \{ integer \},

$\mathrm{K}$ Start \{ integer \},

$\mathrm{K}$ End ( integer \},

Neumann Boundary.

Line $\mathbf{n}$ Direction [ X-Direction / Y-Direction | Z-Direction ]:

Surface [ Top | Bottom I East I West I North I South ] " Surface,"

"Neumann @ Boundary,"

Liquid Mass Flux $\dot{Q}$, \{ real \},

Units $\left[\mathrm{kg} / \mathrm{m}^{\wedge} 2 \mathrm{~s}\left|\mathrm{lbm} / \mathrm{ft}^{\wedge} 2 \mathrm{~s}\right| \mathrm{gm} / \mathrm{cm}^{\wedge} 2 \mathrm{~s}\right.$ ],

Air Mass Fraction $x^{a}\{$ real \},

I Start \{ integer \},

I End ( integer \},

J Start \{ integer \},

J End \{ integer \},

K Start \{ integer \},

$K$ End \{ integer \}, 
Saturated Boundary.

Line n Direction [ X-Direction / Y-Direction | Z-Direction ]:

Surface [ Top I Bottom I East I West I North I South ] " Surface,"

"Saturated @ Boundary,"

,

Liquid Density $\rho_{\ell}$ \{ real \},

Units [ $\mathrm{kg} / \mathrm{m}^{\wedge} 3\left|\mathrm{lbm} / \mathrm{ft}^{\wedge} 3\right| \mathrm{gm} / \mathrm{cm}^{\wedge} 3$ ],

Air Mass Fraction $x_{l}^{a}\{$ real $\}$,

I Start [ integer ],

I End \{ integer \},

J Start \{ integer \},

$J$ End \{ integer \},

$K$ Start $\{$ integer $\}$,

$\mathrm{K}$ End \{ integer \},

Unit-Gradient Boundary.

Line n Direction [ X-Direction / Y-Direction I Z-Direction ]:

Surface [ Top I Bottom I East I West I North I South ] " Surface,"

"Unit Gradient @ Boundary,"

,

Liquid Density $\rho_{\ell}\{$ real $\}$,

Units $\left[\mathrm{kg} / \mathrm{m}^{\wedge} 3\left|\mathrm{lbm} / \mathrm{ft}^{\wedge} 3\right| \mathrm{gm} / \mathrm{cm}^{\wedge} 3\right.$ ],

Air Mass Fraction $x,\{$ real $\}$,

I Start \{ integer \},

I End \{ integer \},

J Start \{ integer \},

$\mathrm{J}$ End [ integer \},

K Start [ integer \},

$\mathrm{K}$ End \{ integer \},

\section{Comments}

Boundary conditions must be applied to active nodes adjacent to exterior boundary surfaces or inactive nodes. The surface orientations refer to a boundary surface with respect to the active node. Dirichlet-type boundary conditions are used to specify liquid pressures at a boundary surface. The phase density and component mass fractions will be used for boundary surface mass fluxes that are directed into the active node. Neumann-type boundary conditions are used to specify liquid-phase mass fluxes at a boundary surface. Flux directions are aligned with the coordinate directions. A positive flux in the $x$ - or $r$-direction is toward the east, in the $y$ - or $\theta$ direction is toward the north, and in the $z$-direction is toward the top. Saturated boundary conditions maintain a zero capillary pressure head at the boundary surface (i.e., liquid pressure is 
maintained equal to the gas pressure). Unit-gradient-type boundary conditions are used to specify surface fluxes at the boundary that result from gravitational body forces only. Unit-gradient-type boundary conditions applied to vertical surfaces are equivalent to zero-flux boundary conditions.

\section{Examples}

A one-dimensional problem requires boundaries to be defined only at the ends of the problem, because the default boundary conditions for undeclared surfaces are zero-flux boundaries. Here a one-dimensional, vertically oriented problem is assigned a constant liquid mass flux in the negative z-direction (the sign of the flux indicates direction) into the domain at the top of the porous medium column and a constant saturation condition (water table) at the bottom.

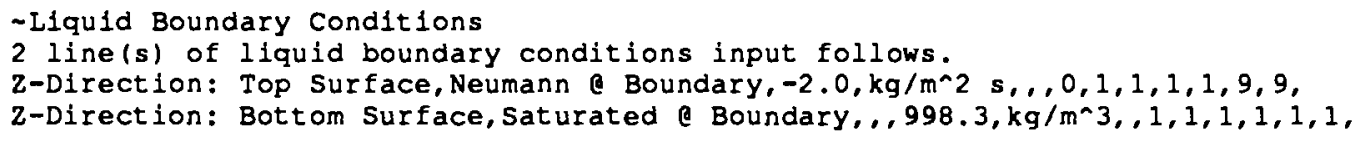




\subsubsection{Gas Boundary Conditions Card}

Function

The Gas Boundary Conditions card is used to specify boundary conditions for gas pressure, gas component mass fractions, gas density, and gas fluxes at the external boundaries of the problem domain and at the boundaries of inactive nodes. Three boundary condition types are available: Dirichlet, Neumann, and unit gradient.

\section{Format}

Line $0 \quad$ "Gas Boundary Conditions"

Line $1 \quad \mathrm{~N}\{$ integer $\}$ " line(s) of gas boundary conditions input follows."

Dirichlet Boundary.

Line $\mathrm{n} \quad$ Direction [ X-Direction / Y-Direction | Z-Direction ]:

Surface [ Top I Bottom I East I West I North I South ] " Surface,"

"Dirichlet @ Boundary,"

Gas Pressure $P_{g}\{$ real $\}$,

Units [ Pa (absolute) | Psi (absolute) | Atm (absolute) | Bar (absolute) ],

Gas Density $\rho_{g}\{$ real $\}$,

Units [ $\mathrm{kg} / \mathrm{m}^{\wedge} 3\left|\mathrm{lbm} / \mathrm{ft}^{\wedge} 3\right| \mathrm{gm} / \mathrm{cm}^{\wedge} 3$ ],

Water-Vapor Mass Fraction $\boldsymbol{x}_{\boldsymbol{g}}^{\boldsymbol{w}}\{\mathrm{real}\}$,

I Start [ integer \},

I End \{ integer \},

J Start \{ integer \},

$J$ End \{ integer \},

$\mathrm{K}$ Start $\{$ integer $\}$,

$\mathrm{K}$ End \{ integer \},

Neumann Boundary.

Line $\mathrm{n}$ Direction [ X-Direction / Y-Direction / Z-Direction ]:

Surface [ Top | Bottom I East I West I North I South ] " Surface,"

"Neumann@ Boundary,"

Gas Mass Flux $\dot{Q}_{g}\{$ real $\}$,

Units $\left[\mathrm{kg} / \mathrm{m}^{\wedge} 2 \mathrm{~s}\left|\mathrm{lbm} / \mathrm{ft}^{\wedge} 2 \mathrm{~s}\right| \mathrm{gm} / \mathrm{cm}^{\wedge} 2 \mathrm{~s}\right.$ ],

,

Water-Vapor Mass Fraction $x_{g}^{w}\{$ real $\}$,

I Start \{ integer \},

I End \{ integer \},

J Start \{ integer \},

$\mathrm{J}$ End [ integer \},

K Start ( integer \},

$\mathrm{K}$ End \{ integer \}, 
Unit-Gradient Boundary.

Line n Direction [ X-Direction / Y-Direction / Z-Direction ]:

Surface [ Top I Bottom I East I West | North I South ] " Surface,"

"Unit Gradient @ Boundary,"

,

Gas Density $\rho_{g}$ ( real ),

Units [ $\mathrm{kg} / \mathrm{m}^{\wedge} 3\left|\mathrm{lbm} / \mathrm{ft}^{\wedge} 3\right| \mathrm{gm} / \mathrm{cm}^{\wedge} 3$ ],

Water-Vapor Mass Fraction $x_{g}^{w}$ ( real \},

I Start \{ integer \},

I End ( integer \},

J Start \{ integer \},

J End \{ integer \},

K Start \{ integer \},

$\mathrm{K}$ End $\{$ integer \},

\section{Comments}

Boundary conditions must be applied to active nodes adjacent to exterior boundary surfaces or inactive nodes. The surface orientations refer to a boundary surface with respect to the active node. Dirichlet-type boundary conditions are used to specify gas pressures at a boundary surface. The phase density and component mass fractions will be used for boundary surface mass fluxes that are directed into the active node. Neumann-type boundary conditions are used to specify gasphase mass fluxes at a boundary surface. Flux directions are aligned with the coordinate directions. A positive flux in the $x$ - or $r$-direction is toward the east, in the $y$ - or $\theta$-direction is toward the north, and in the $z$-direction is toward the top. Unit-gradient-type boundary conditions are used to specify surface fluxes at the boundary that result from gravitational body forces only. Unit-gradient-type boundary conditions applied to vertical surfaces are equivalent to zero-flux boundary conditions.

\section{Examples}

The first example is for a problem that does not require a gas boundary condition to be defined:

\footnotetext{
-Gas Boundary Conditions

o line(s) of gas boundary conditions input follows.
}

A common gas boundary condition is constant atmospheric pressure. Here, a Dirichlet boundary is defined on the west surface of a problem domain with constant gas pressure of $101,330 \mathrm{~Pa}$, gas density at this boundary of $0.91944 \mathrm{~kg} / \mathrm{m}^{3}$, and vapor mass fraction 0.21395 : 
- Gas Boundary Conditions

1 line(s) of gas boundary conditions input follows.

X-Direction: West Surface,Dirichlet e Boundary, 101330.0, Pa (absolute) , 0.91944, kg/m³,0.21395, 1, 1, 1, 1, 1, 1, 


\subsubsection{Vapor Boundary Conditions Card}

\section{Function}

The Vapor Boundary Conditions card specifies boundary conditions only with respect to diffusion of components through the gas phase; the advection of components in the gas phase at boundary surfaces is specified through gas boundary conditions. Vapor boundary conditions can be declared at the external boundary surfaces of the problem domain and at the boundaries between active and inactive nodes. Only Dirichlet-type boundary conditions are available for vapor boundary conditions.

\section{Format}

Line $0 \quad$ "Vapor Boundary Conditions"

Line $1 \quad \mathrm{~N}\{$ integer \} " line(s) of vapor boundary conditions input follows."

\section{Dirichlet Boundary.}

Line $\mathrm{n} \quad$ Direction [ X-Direction I Y-Direction | Z-Direction ]:

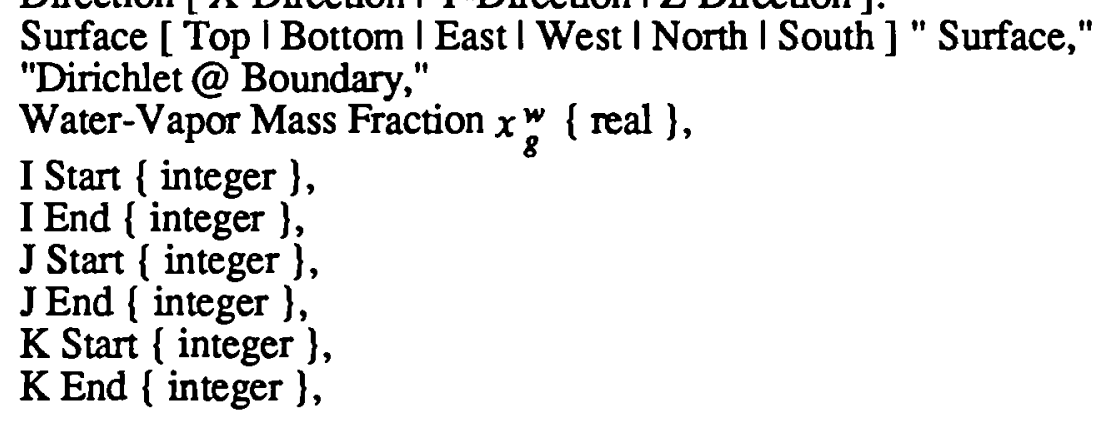

\section{Comments}

Boundary conditions must be applied to active nodes adjacent to exterior boundary surfaces or inactive nodes. The surface orientations refer to a boundary surface with respect to the active node. Dirichlet-type boundary conditions are used to specify water-vapor mass fraction at a boundary surface. Water-vapor mass fractions declared through the Vapor Boundary Conditions card are used only to compute the diffusion of water vapor across a boundary surface. Advection of water vapor across a boundary surface is controlled through the Gas Boundary Conditions card. Normally, the water-vapor mass fractions declared on the Vapor Boundary Conditions and Gas Boundary Conditions cards will be equivalent. 


\section{Examples}

If no vapor boundary conditions are to be declared in an "input" file, the Vapor Boundary Conditions card would be entered as

- Vapor Boundary Conditions

0 line(s) of vapor boundary conditions input follows.

In the next example, the Vapor Boundary Conditions card is provided for a model domain with an upper boundary that is the interface with the atmosphere. The upper boundary is a sloping surface, so inactive nodes (refer to Section 6.2.2 for information on inactive node declaration) are used to represent the atmosphere, and the boundary is defined at the edge of the inactive region. The vapor boundary condition in this example is a constant vapor mass fraction of 0.003 .

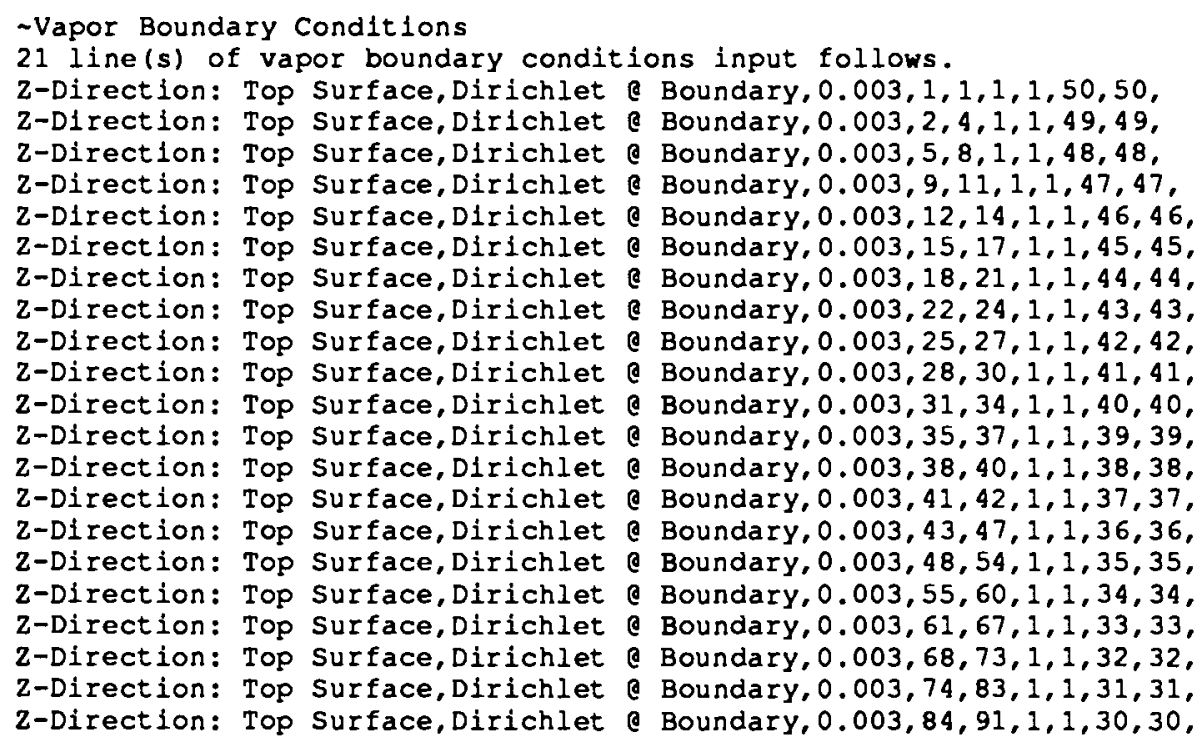




\subsubsection{Energy Boundary Conditions Card}

\section{Eunction}

The Energy Boundary Conditions card specifies boundary conditions for temperature, volumetric enthalpies for liquid and gas, and heat fluxes at the external boundaries of the problem domain and at the boundaries of inactive nodes (if any). Two boundary condition types are available: Dirichlet and Neumann.

Format

Line 0 "-Energy Boundary Conditions"

Line $1 \quad \mathrm{~N}$ ( integer \} " line(s) of energy boundary conditions input follows."

Dirichlet Boundary.

Line n Direction [ X-Direction / Y-Direction | Z-Direction ]:

Surface [ Top I Bottom I East I West I North I South ] " Surface,"

"Dirichlet @ Boundary,"

Temperature $T$ \{ real \},

Units [ Celsius I Fahrenheit | Rankine I Kelvin ],

Liquid Volumetric Enthalpy $h_{\ell}\{$ real \},

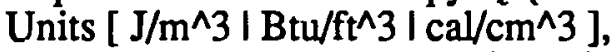

Gas Volumetric Enthalpy $h_{8}\{$ real $\}$,

Units [ J/m^3| Btu/ft^3| $\left.\mathrm{cal}^{\wedge} / \mathrm{cm}^{\wedge} 3\right]$,

I Start ( integer \},

I End \{ integer \},

J Start \{ integer \},

J End \{ integer \},

K Start \{ integer \},

$\mathrm{K}$ End $\{$ integer \}, 
Neumann Boundary.

Line n Direction [ X-Direction I Y-Direction I Z-Direction ]:

Surface [ Top I Bottom I East I West I North I South ] " Surface,"

"Neumann@ Boundary,"

Heat Flux $\dot{Q}_{\mathrm{e},:}\{$ real $\}$,

Units [ W/m^2 I Btu/h ft^2l cal $/ \mathrm{cm}^{\wedge} 2 \mathrm{~s}$ ],

Liquid Volumetric Enthalpy $h_{\ell}\{$ real $\}$,

Units [ J/m^3| Btu/ft^3 I cal/cm^3],

Gas Volumetric Enthalpy $h_{g}\{$ real $\}$,

Units $\left[\mathrm{J} / \mathrm{m}^{\wedge} 3\left|\mathrm{Btu} / \mathrm{ft}^{\wedge} 3\right| \mathrm{cal} / \mathrm{cm}^{\wedge} 3\right]$,

I Start $\{$ integer $\}$,

I End \{ integer \},

J Start \{ integer \},

$J$ End \{ integer \},

K Start $\{$ integer $\}$,

$\mathrm{K}$ End $\{$ integer $\}$,

\section{Comments}

Boundary conditions must be applied to active nodes adjacent to exterior boundary surfaces or inactive nodes. The surface orientations refer to a boundary surface with respect to the active node. Dirichlet-type boundary conditions are used to specify temperatures at a boundary surface. The liquid and gas volumetric enthalpies will be used for boundary surface mass fluxes that are directed into the active node. Neumann-type boundary conditions are used to specify heat fluxes at a boundary surface. Flux directions are aligned with the coordinate directions. A positive flux in the $x$ - or $r$-direction is toward the east, in the $y$ - or $\theta$-direction is toward the north, and in the $z$ direction is toward the top.

\section{Examples}

These are the boundary conditions for a one-dimensional, horizontally oriented heat pipe problem (see Section 8.0 for a complete description of the heat pipe problem). The east side is assigned a constant energy flux of $100 \mathrm{~W} / \mathrm{m}^{2}$ directed into the domain (the negative sign indicates the direction of the flux) while the west side is assigned a constant temperature of $70^{\circ} \mathrm{C}$.

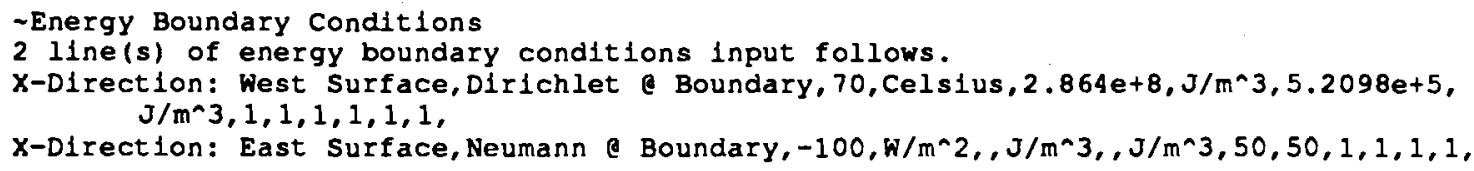


Any boundary condition can be applied either to the domain boundary or to the surface of inactive nodes (see Section 6.2.2 for information on declaring inactive nodes). In the following example of an Energy Boundary Conditions card, a boundary at a constant temperature of $20^{\circ} \mathrm{C}$ is assigned to the top of a two-dimensional $(x-z), 5$ by 5 node domain that has inactive nodes in the lower left-hand side. A constant temperature of $30^{\circ} \mathrm{C}$ is assigned to the top of each inactive node that is adjacent to a computational node to form a diagonal boundary in the problem.

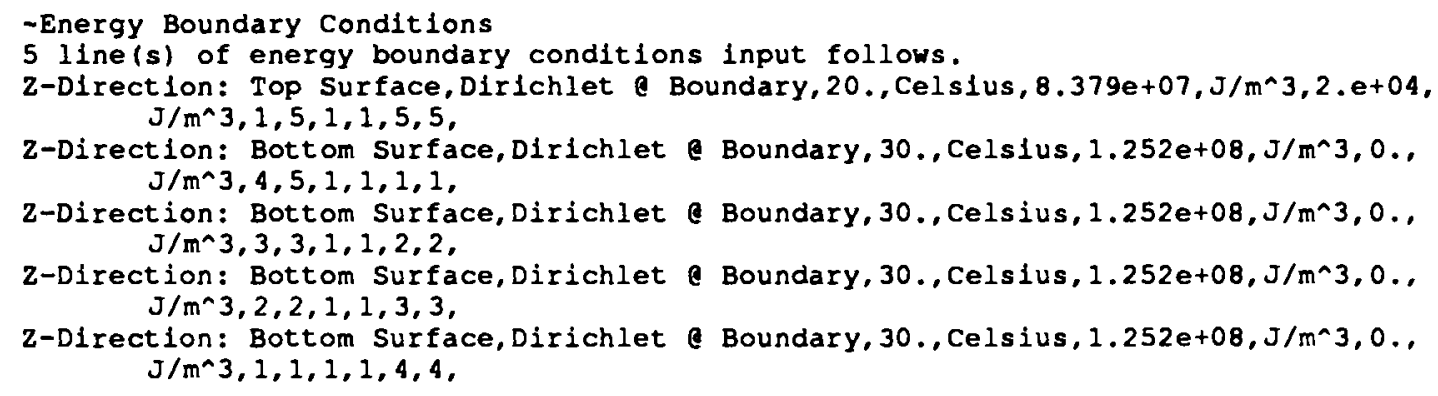




\subsubsection{Species Boundary Conditions Card}

Function

The Species Boundary Conditions card specifies boundary conditions for species concentrations in the aqueous and gas phases and species boundary surface fluxes individually for the aqueous- and gas-phase fluxes at the external boundaries of the problem domain and at the boundaries of inactive nodes. Two boundary condition types are available: Dirichlet and Neumann.

\section{Format}

Line $0 \quad$ "Species Boundary Conditions"

Line $1 \quad \mathrm{~N}$ \{ integer \} " line(s) of species boundary conditions input follows."

Dirichlet Boundary.

Line $n \quad$ Direction [ X-Direction / Y-Direction / Z-Direction ]:

Surface [ Top I Bottom I East I West I North I South ] " Surface,"

"Dirichlet@Boundary,"

Liquid-Phase Species Concentration $C_{\ell}\{$ real $\}$,

Units [ (mol;Ci)/m^3 I (mol;Ci)/f^^3 I (mol;Ci)/liter I (mol;Ci)/cm^3) ], Gas-Phase Species Concentration $C_{g}\{$ real $\}$,

Units [ (mol;Ci)/m^3 I (mol;Ci)/ft^3| (mol;Ci)/liter I (mol;Ci)/(cm^3) ],

I Start [ integer ],

I End \{ integer \},

J Start $\{$ integer $\}$,

$\mathrm{J}$ End \{ integer \},

$\mathrm{K}$ Start $\{$ integer $\}$,

$\mathrm{K}$ End ( integer \},

Neumann Boundary.

Line n Direction [ X-Direction | Y-Direction | Z-Direction ]:

Surface [ Top I Bottom I East I West I North I South ] " Surface,"

"Neumann@ Boundary,"

Liquid-Phase Species Flux $\dot{Q}_{c}$ \{real \},

Units $\left.\left[(\mathrm{mol} ; \mathrm{Ci}) / \mathrm{m}^{\wedge} 2 \mathrm{~s} \mathrm{l}(\mathrm{mol} ; \mathrm{Ci}) / \mathrm{ft}^{\wedge} 2 \mathrm{sl}(\mathrm{mol} ; \mathrm{Ci}) / \mathrm{cm}^{\wedge} 2 \mathrm{~s}\right)\right]$,

Gas-Phase Species Flux $\dot{Q}_{c_{\varepsilon}}\{$ reä \},

Units $\left[(\mathrm{mol} ; \mathrm{Ci}) / \mathrm{m}^{\wedge} 2 \mathrm{~s} \mid(\mathrm{mol} ; \mathrm{Ci}) / \mathrm{ft}^{\wedge} 2 \mathrm{~s}\left((\mathrm{~mol} ; \mathrm{Ci}) / \mathrm{cm}^{\wedge} 2 \mathrm{~s}\right)\right]$,

I Start $\{$ integer $\}$,

I End \{ integer \},

J Start $\{$ integer $\}$,

$\mathrm{J}$ End \{ integer \},

K Start \{ integer \},

$\mathrm{K}$ End \{ integer \}, 


\section{Comments}

Boundary conditions must be applied to active nodes adjacent to exterior boundary surfaces or inactive nodes. The surface orientations refer to a boundary surface with respect to the active node. Dirichlet-type boundary conditions are used to specify species concentrations at a boundary surface. Neumann-type boundary conditions are used to specify species fluxes at a boundary surface. Flux directions are aligned with the coordinate directions. A positive flux in the $x$ - or $r$ direction is toward the east, in the $y$ - or $\theta$-direction is toward the north, and in the $z$-direction is toward the top.

\section{Examples}

A two-dimensional, horizontally oriented, 75-node transport problem with constant concentrations at each end ( $1.0 \mathrm{~mol} /$ liter at the west side, $0.1 \mathrm{~mol} / \mathrm{liter}$ at the east side) is entered as

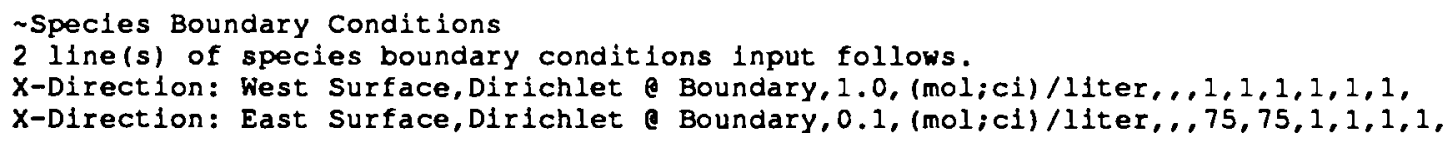

If default boundary conditions (zero-flux) are desired, or the species transport equation is not activated, then the Species Boundary Conditions card is entered as

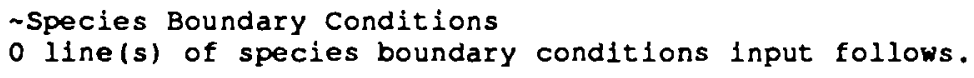




\subsection{Initial Conditions and Sources}

The initial conditions for a simulation problem can be specified either through a "restart" file or with the Initial Conditions card. When the initial conditions are specified with a "restart" file, information on the Initial Conditions card is ignored. The Initial Conditions card allows users to specify values for the primary variables and Darcy velocities, when appropriate. For added flexibility, the Initial Conditions card provides three methods for specifying the liquid pressure, gas pressure, and liquid saturation. Because the soil-moisture-retention functions relate these variables, only two of three variables are required to completely specify the hydrologic state of the system. For example, the user may specify the liquid saturation and gas pressure on the Initial Conditions card, and the corresponding liquid pressure field would be computed internally by MSTS. Alternatively, the gas pressure and liquid pressure could be specified and MSTS would compute the corresponding liquid saturation.

The Sources and Sinks card is used to define sources or sinks for water mass, air mass, thermal energy, and species mass or concentration. Sources may be defined as constant for a certain time period or may vary following a table of values as a function of time. Each source is defined with respect to a rectangular range of source node(s), though multiple Sources and Sinks cards can be used to give geometric flexibility in defining a source. 


\subsubsection{Initial Conditions Card}

\section{Function}

The Initial Conditions card is used to specify the values of field variables at computational nodes at the start of a simulation (time zero). No primary variable initial conditions are required for restart simulations. Field variables are defined at node points, whereas field velocities or fluxes are defined on node surfaces. The field velocity associated with a particular node index is defined for the lower surface (e.g., west, south, bottom). For example, $x$-direction velocities defined at a node with $(I, J, K)$ indices refers to the $x$-direction velocity at the west surface of the node with the same indices. Uncomputed velocity variables must be specified for both normal and restart simulations. The Initial Saturation option declares which two variables among the liquid pressure, gas pressure, and liquid saturation will be used to specify the initial hydrologic conditions.

Format

Line $0 \quad$ "Initial Conditions"

Line 1 Initial Saturation [ Liquid Saturation \& Gas Pressure I Liquid Saturation \& Liquid Pressure l Gas Pressure \& Liquid Pressure ]

Line $2 \quad \mathrm{~N}$ \{ integer \} " line(s) of initial conditions input follows."

Line n Field Variable Name [ Liquid Pressure I Gas Pressure I Liquid Saturation I

Temperature I Species Concentration I X-Dir. Liquid Velocity I Y-Dir. Liquid Velocity I Z-Dir. Liquid Velocity I X-Dir. Gas Velocity | Y-Dir. Gas Velocity | Z-Dir. Gas Velocity I Vapor Mass Fraction ],

Field Variable Value \{ real \},

Units Pressure. [ Pa (absolute) | Psi (absolute) | Atm (absolute) | Bar (absolute)], Saturation. (dimensionless),

Temperature. [ Celsius I Fahrenheit I Rankine I Kelvin ],

Concentration. [ (mol;ci)/m^3 I (mol;ci)/ft^3 I (mol;ci)/liter I (mol;ci)/cm^3 ]

Velocity. [ $\mathrm{m} / \mathrm{s}|\mathrm{ft} / \mathrm{s}| \mathrm{cm} / \mathrm{s}|\mathrm{m} / \mathrm{h}| \mathrm{ft} / \mathrm{h} \mid \mathrm{cm} / \mathrm{h} \mathrm{I} \mathrm{m} /$ day $\mid \mathrm{ft} /$ day $\mid \mathrm{cm} /$ day $]$,

Vapor Mass Fraction. (dimensionless),

$x$-Direction Gradient \{ real \},

Units [ $1 / \mathrm{m} \mathrm{|} 1 / \mathrm{ft} \mathrm{I} 1 / \mathrm{cm}]$,

$y$-Direction Gradient \{ real \},

Units [ $1 / \mathrm{m} \mathrm{|} 1 / \mathrm{ft} \mid 1 / \mathrm{cm}]$,

$z$-Direction Gradient $\{$ real $\}$,

Units [ $1 / \mathrm{m} \mathrm{|1/ft} \mathrm{I} \mathrm{1/cm} \mathrm{],}$

I Start $\{$ integer \},

I End [ integer \},

J Start $\{$ integer $\}$,

$\mathrm{J}$ End \{ integer \},

K Start \{ integer \},

$\mathrm{K}$ End \{ integer \}, 


\section{Comments}

Unless the simulation is beginning from a "restart" file, primary variable initial conditions must be specified. Default initial conditions for the primary variables are the following: liquid pressure $(101325 \mathrm{~Pa})$, gas pressure $(101325 \mathrm{~Pa})$, temperature $\left(20^{\circ} \mathrm{C}\right)$, and species concentration $\left(0 \mathrm{~mol} / \mathrm{m}^{3}\right)$. Default initial conditions for the secondary variables are the following: liquid saturation $\left(0 \mathrm{~m}^{3} / \mathrm{m}^{3}\right)$ and vapor mass fraction (thermodynamic saturation conditions for two-phase conditions, $0 \mathrm{~kg} / \mathrm{kg}$ for gas-phase-only conditions). Default initial conditions for Darcy velocities are $0 \mathrm{~m} / \mathrm{s}$. Before being used in MSTS, initial conditions are checked for thermodynamic consistency and operational limits. The following restrictions apply to the primary variable initial conditions: liquid pressure (611.25 $\left.\mathrm{Pa} \leq P_{\ell} \leq 2.212 \times 10^{7} \mathrm{~Pa}\right)$, gas pressure (611.25 $\mathrm{Pa} \leq P_{g} \leq$ $\left.2.212 \times 10^{7} \mathrm{~Pa}\right)$, temperature $\left(0.01^{\circ} \mathrm{C} \leq T \leq 374.14^{\circ} \mathrm{C}\right)$. Thermodynamic consistency checks are performed on two-phase nodes for evaporation and on gas-phase-only nodes for condensation. To satisfy thermodynamic equilibrium conditions for two-phase nodes (i.e., nodes with both liquid and gas phases present), the specified gas pressure must be greater than the saturated water-vapor partial pressure. To satisfy thermodynamic equilibrium conditions for gas-phase-only nodes (i.e., nodes with only the gas phase present), the specified vapor mass fraction must be less than the saturated vapor mass fraction. If either of these conditions is violated, the simulation is aborted, with an error message printed to the "output" file.

Initial species concentrations are expressed in terms of the total volume, rather than gas- or aqueous-phase volumes. For example, initial concentrations expressed per unit aqueous-phase volume must be converted to concentrations per unit total volume by multiplying the aqueous-phase concentration by the product of the diffusive porosity $\left(n_{D}\right)$ and the liquid saturation under initial conditions $\left(s_{\ell}\right)$.

To simplify the specification of initial conditions, the Initial Conditions card accepts initial conditions expressed in terms of linear gradients. Gradients are expressed in terms of dimensional length and start at the node position of the node indexed on the Initial Conditions card. An excellent example of specifying initial conditions in terms of gradients results from the specification of an initial liquid saturation field under static equilibrium conditions. For a simulation involving the Water mode, specifying a constant gradient to the liquid pressure field that decreases with elevation by the product of the aqueous-phase density and acceleration of gravity $(-9793.5 \mathrm{~Pa} / \mathrm{m} @$ $20^{\circ} \mathrm{C}$ ) will result in an initial liquid saturation field at static equilibrium. The water table height for such initial conditions will be located at the point of zero capillary pressure. 


\section{Examples}

The initial condition for a 100 -node vertically oriented, one-dimensional problem is atmospheric gas pressure and a liquid pressure of $42000 \mathrm{~Pa}$ (absolute) throughout. The Initial Conditions card for this case appears as

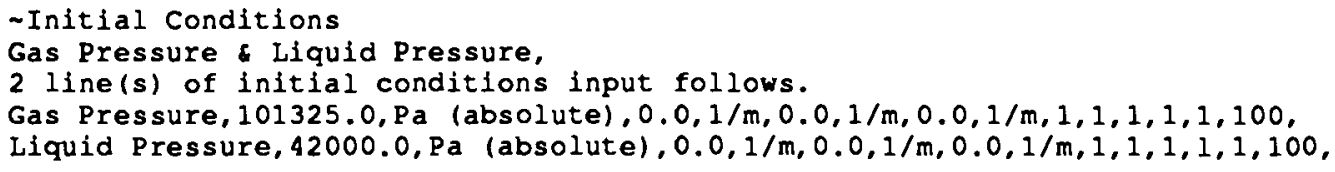

The initial condition is not restricted to a single value for the whole problem domain. One way to vary the condition is by specifying different values for different ranges of nodes. For example, the previous card can be modified to give the same liquid pressure at time zero for the lower half of the nodes, but a lower pressure for the upper half:

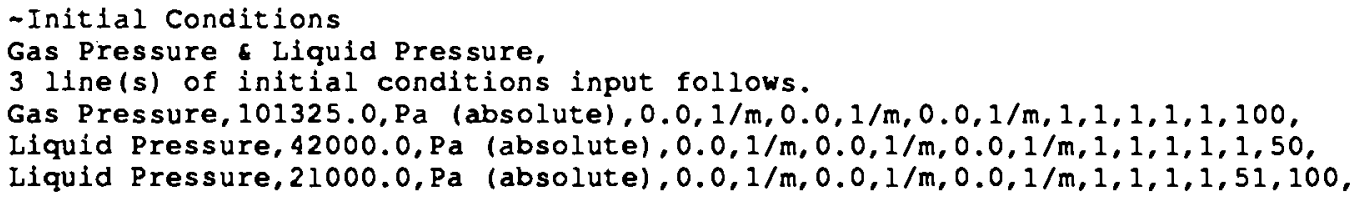

If a uniform variation of initial conditions is desired, use the gradient utility. For example, if the initial conditions of a one-dimensional, 10-m-long, horizontally oriented problem, with a uniform grid, vary linearly from zero liquid saturation at the left side to fully saturated at the right side, the initial condition could be specified as

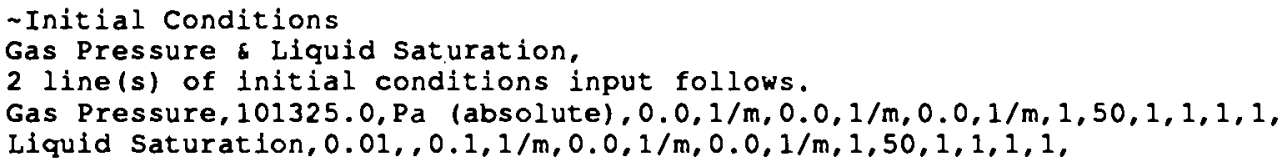

which would cause the initial condition of zero saturation at the west boundary to be increased 0.1 per meter until reaching 1.0 (fully saturated) at $10 \mathrm{~m}$, which is the east boundary. The gradient feature may be used with any initial condition in any dimension(s). 


\subsubsection{Sources and Sinks Card}

\section{Function}

The Sources and Sinks card is used to identify the location and strength of sources and/or sinks of liquid mass, air mass, thermal energy, and species mass. The strength of a source or sink either may be constant in time or may vary following tabular input.

\section{Format}

Line $0 \quad$ "Sources \& Sinks"

Line $1 \quad \mathrm{~N}$ ( integer \} " line(s) of sources \& sinks input follows."

Line n Source Type [ Liquid Mass I Gas Mass I Thermal Energy I Species I Species Concentration ], 
Liquid Mass.

Source Variation [ Constant I Tabular ],

Constant.

Liquid Mass Source Rate $\dot{m},\{$ real \},

Units [ kg/s | lbm/s | gm/s | kg/h I lbm/h $|\mathrm{gm} / \mathrm{h}| \mathrm{kg} /$ day $\mid \mathrm{lbm} /$ day $\mid \mathrm{gm} /$ day $]$,

Liquid Enthalpy $h_{\ell}$ \{ real \},

Units [ J/kg I Btu/lbm I cal/gm ],

Air Mass Fraction $x_{p}^{a}$, ( real \},

Source Start Time \{ real \},

Units [ $\mathrm{s}|\mathrm{min}| \mathrm{h} \mid$ day $|\mathrm{wk}| \mathrm{yr}$ ],

Source End Time \{ real \},

Units [ $s|\min | \mathrm{h} \mid$ day $\mid$ wk $\mid$ yr ],

I Start \{ integer \},

I End \{ integer \},

J Start \{ integer \},

$\mathrm{J}$ End [ integer \},

K Start \{ integer \},

$\mathrm{K}$ End \{ integer \},

Tabular.

Table Number \{ integer \},

Line n M $\quad$ \{ integer $\}$ "line(s) of source tables input follows."

Line $m$ Time $\{$ real $\}$,

Units [ $\mathrm{s}|\mathrm{min}| \mathrm{h} \mid$ day $|\mathrm{wk}| \mathrm{yr}$ ],

Liquid Mass Source Rate $\dot{m}_{\ell}\{$ real \},

Units [ kg/s | lbm/s I gm/s | kg/h I lbm/h I gm/h | kg/day | lbm/day I gm/day ], Liquid Enthalpy $h_{\ell}\{$ real $\}$,

Units [ J/kg I Btu/lbm I cal/gm ],

Air Mass Fraction \{ real \}, 
Gas Mass.

Source Variation [ Constant I Tabular ],

Constant.

Gas Mass Source Rate $\dot{m}_{g}\{$ real \},

Units [ kg/s $|\mathrm{lbm} / \mathrm{s}| \mathrm{gm} / \mathrm{s}|\mathrm{kg} / \mathrm{h}| \mathrm{lbm} / \mathrm{h}|\mathrm{gm} / \mathrm{h}| \mathrm{kg} /$ day $\mid \mathrm{lbm} /$ day $\mid \mathrm{gm} /$ day ], Gas Enthalpy $h_{g}$ ( real \},

Units [ J/kg I Btu/lbm I cal/gm ],

Vapor Mass Fraction $x_{g}^{w}\{$ real $\}$,

Source Start Time \{ real \},

Units $[s|\min | \mathrm{h} \mid$ day $|\mathrm{wk}| \mathrm{yr}$ ],

Source End Time \{real \},

Units [ $\mathrm{s}|\min | \mathrm{h} \mid$ day $|\mathrm{wk}| \mathrm{yr}$ ],

I Start \{ integer \},

I End ( integer \},

J Start \{ integer \},

J End [ integer \},

K Start \{ integer \},

$\mathrm{K}$ End \{ integer \},

Tabular.

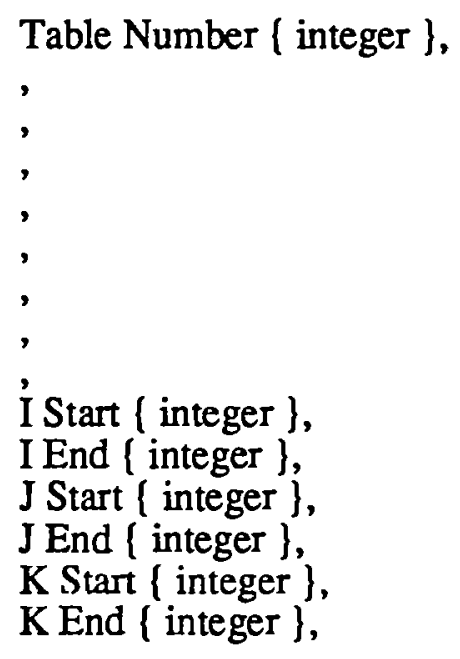

Line n M \{ integer \} "line(s) of source tables input follows."

Line $m$ Time [ real \},

Units [ $s|\min | \mathrm{h} \mid$ day $|\mathrm{wk}| \mathrm{yr}$ ],

Gas Mass Source Rate $\dot{m}_{g}$ \{real \},

Units [ $\mathrm{kg} / \mathrm{s}|\mathrm{lbm} / \mathrm{s}| \mathrm{gm} / \mathrm{s}|\mathrm{kg} / \mathrm{h}| \mathrm{lbm} / \mathrm{h}|\mathrm{gm} / \mathrm{h}| \mathrm{kg} /$ day $\mid \mathrm{lbm} /$ day $\mid \mathrm{gm} /$ day ],

Gas Enthalpy $h_{g}\{$ real \},

Units [ J/kg I Btu/lbm I cal/gm ],

Vapor Mass Fraction [ real \}, 
Thermal Energy.

Source Variation [ Constant I Tabular ],

Constant.

Energy Source Rate $\dot{Q}$ \{ real \},

Units $\left[\mathrm{W} / \mathrm{m}^{\wedge} 3\left|\mathrm{Btu} / \mathrm{h} \mathrm{ft} \mathrm{ft}^{\wedge}\right| \mathrm{cal} / \mathrm{s} \mathrm{cm} \mathrm{cm}^{\wedge} 3\right.$,

,

Source Start Time ( real \},

Units [ $s|\min | \mathrm{h} \mid$ day $|\mathrm{wk}| \mathrm{yr}$ ],

Source End Time \{ real \},

Units [ $s|\min | \mathrm{h} \mid$ day $|\mathrm{wk}| \mathrm{yr}$ ],

I Start [ integer \},

I End \{ integer \},

J Start \{ integer \},

$\mathrm{J}$ End \{ integer \},

K Start \{ integer \},

$\mathrm{K}$ End ( integer \},

Tabular.

Line $n$

Table Number \{ integer \},

,

,

,

I Start ( integer \},

I End [ integer \},

J Start \{ integer \},

$\mathrm{J}$ End \{ integer \},

K Start \{ integer \},

$\mathrm{K}$ End \{ integer \},

Line $m$ Time $\{$ real $\}$,

Units [ $s|\min | \mathrm{h} \mid$ day $|\mathrm{wk}| \mathrm{yr}$ ],

Energy Source Rate \{ real \},

Units $\left[\mathrm{W} / \mathrm{m}^{\wedge} 3|\mathrm{Btu} / \mathrm{h} \mathrm{ft} \wedge 3| \mathrm{cal} / \mathrm{s} \mathrm{cm}^{\wedge} 3\right.$ ],

,

,

, 
Species.

Source Variation [ Constant I Tabular ],

Constant.

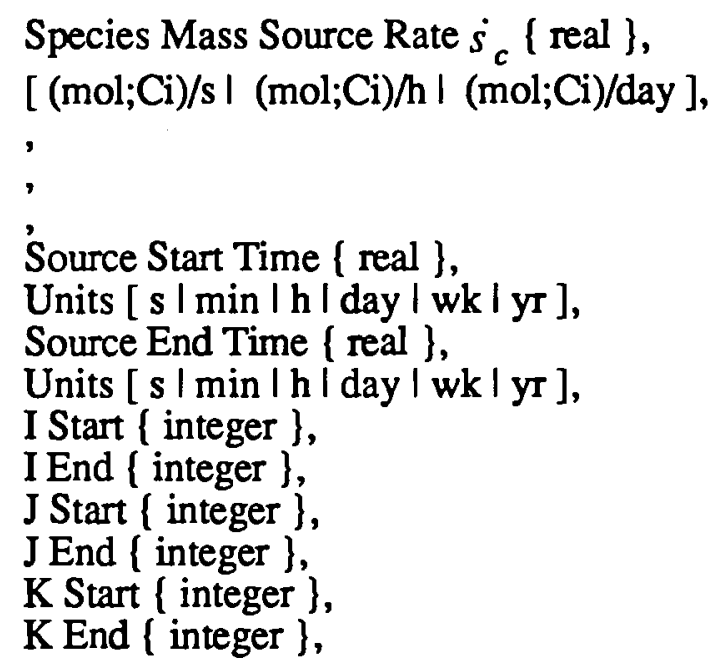

Tabular.

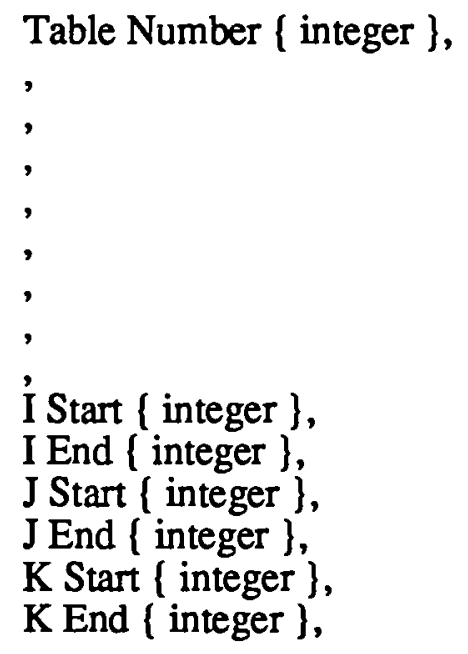

Line $n \quad M$ \{ integer \} " line(s) of source tables input follows."

Line $m$ Time $\{$ real $\}$,

Units [ $\mathrm{s}|\mathrm{min}| \mathrm{h} \mid$ day $|\mathrm{wk}| \mathrm{yr}$ ],

Species Mass Source Rate $\dot{s}_{c}\{$ real \}, [ (mol;Ci)/s | (mol;Ci)/h I (mol;Ci)/day ], 
Species Concentration.

Source Variation [ Constant I Tabular ],

Constant.

Species Mass Concentration Source Rate $\dot{s}_{c, v}\{$ real $\}$, [ (mol; $\mathrm{Ci}) / \mathrm{m}^{\wedge} 3 \mathrm{~s}\left|(\mathrm{~mol} ; \mathrm{Ci}) / \mathrm{m}^{\wedge} 3 \mathrm{~h}\right|(\mathrm{mol} ; \mathrm{Ci}) / \mathrm{m}^{\wedge} 3$ day $]$,

,

,

Source Start Time \{ real \},

Units [ $s|\min | \mathrm{h} \mid$ day $|\mathrm{wk}| \mathrm{yr}$ ],

Source End Time \{ real \},

Units $[s|\min | \mathrm{h} \mid$ day $|\mathrm{wk}| \mathrm{yr}$ ],

I Start \{ integer \},

I End \{ integer \},

J Start \{ integer \},

$J$ End \{ integer \},

K Start \{ integer \},

$\mathrm{K}$ End \{ integer \},

Tabular.

Table Number $\{$ integer $\}$,

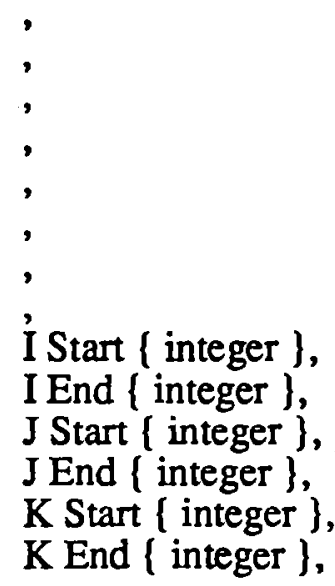

Line n $\quad M\{$ integer $\}$ "line(s) of source tables input follows."

Line $m$ Time $\{$ real $\}$,

Units [ $s|\min | \mathrm{h} \mid$ day $|\mathrm{wk}| \mathrm{yr}$ ],

Species Mass Concentration Source Rate $\dot{s}_{c}$ \{real \},

[ (mol; $\mathrm{Ci}) / \mathrm{m}^{\wedge} 3 \mathrm{~s}\left|(\mathrm{~mol} ; \mathrm{Ci}) / \mathrm{m}^{\wedge} 3 \mathrm{~h}\right|(\mathrm{mol} ; \mathrm{Ci}) / \mathrm{m}^{\wedge} 3$ day $]$,

,

,

, 


\section{Comments}

The source quantity (liquid mass, gas mass, thermal energy, or species mass) is per grid cell over the range of grid nodes identified by the I Start, I End, J Start, J End, K Start, and K End integer fields. Therefore, if $1.0 \mathrm{~kg} / \mathrm{s}$ of liquid mass is the source for the range of nodes $(1,3,1,1,1,1)$, then a total of $3.0 \mathrm{~kg} / \mathrm{s}$ of mass will be introduced to the problem domain. Source term rates between tabular inputs are obtained with linear interpolation; therefore, the integrated source quantity will be dependent on the simulation time step. Strongly varying source terms will require smaller time steps to accurately model the integrated source quantity.

Enthalpy inputs associated with the liquid and gas mass sources are used only if the solution option includes an active energy equation. If the energy equation is inactive (i.e., unsolved), then these values are unused and blank entries may be used. Enthalpy inputs include both water and air components for the gas phase, but only water components for the liquid phase. Component enthalpies are combined by mass fraction weighting. The reference point for water enthalpy is liquid water at $0.01^{\circ} \mathrm{C}$ whereas the reference point for air enthalpy is gaseous air at $0^{\circ} \mathrm{C}$.

Species "mass" may represent either the number of moles of the substance (e.g., mol/s represents the number of moles introduced per second) or the activity of a radionuclide, expressed in curies (Ci). Input and output are marked in both units (e.g., mol;Ci/s). The actual unit is based on the use of the code, so that if the input reflects activity, so does the output.

Species sources may be expressed as either concentration per unit time or mass per unit time. Sources expressed as concentration per unit time are usually preferred for source regions comprising several grid nodes of varying size, because MSTS will introduce a mass source equally to nodes regardless of cell volume. This would result in artificial concentration variation due purely to cell volume. This is not a problem if all grid cells in the source region (defined by I Start, I End, J Start, J End, $\mathrm{K}$ Start, and $\mathrm{K}$ End) are the same volume or if only one node is defined as a source region.

\section{Examples}

A problem that has no internal sources or sinks will have a Sources and Sinks card that appears as

rources sinks

0 line(s) of sources sinks input follows. 
The following example is for a problem with a time-varying source that releases nothing at time zero and increases the release by an additional $1.0 \mathrm{Ci} / \mathrm{m}^{3} \mathrm{~s}$ each year for a period of 5 years. The time variation of the source is expressed in tabular input (Table 1).

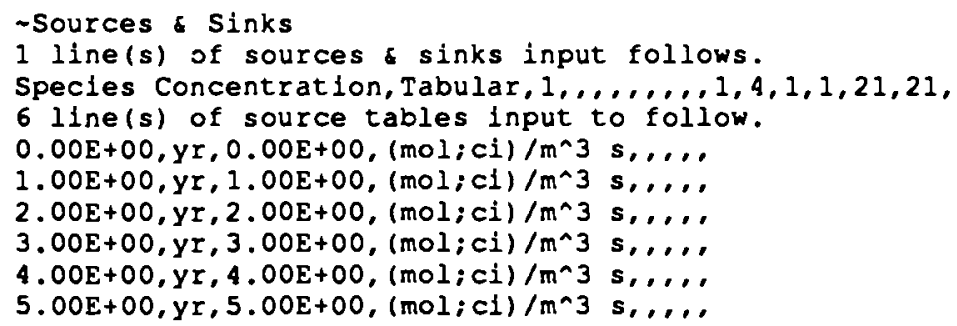

A thermal-energy source of $20,000 \mathrm{~W} / \mathrm{m}^{3}$ that is constant from time zero to 1875 seconds for a single grid node might look like the last example:

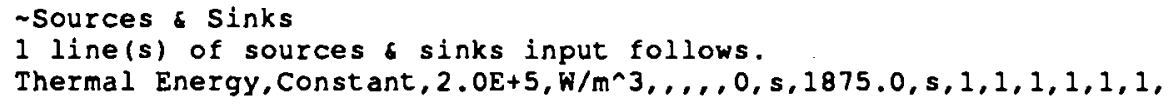




\subsection{Output Control}

Output control occurs in MSTS using the Liquid Pathline Trace card, Output Control card, and Surface Flux Integrator card. The Liquid Pathline Trace card invokes an algorithm for groundwater travel time that is based on the mean velocity of water though the model domain. The starting coordinates of a pathline are defined along with the circumstances under which to cease a trace, such as reaching a certain plane or crossing a specified distance. The Output Control card is used to indicate precisely what field variables should be recorded at what times and over what range of computational nodes. Separate output can be requested for the "output" and "plot" files, and for reference node reporting during a simulation execution. The Surface Flux Integrator card is useful for obtaining the flux of liquid mass, gas mass, species mass or concentration, or energy across specified planes. 


\subsubsection{Liquid Pathline Trace Card}

\section{Function}

The Liquid Pathline Trace card is used to define pathline beginning locations and termination conditions to be traced by MSTS to produce the pathline geometry and characteristic travel times based on mean liquid velocities.

\section{Format}

Line 0

Line 1

Line $n$
" Liquid Pathline Trace"

$\mathrm{N}\{$ integer \} " line(s) of liquid pathline trace input follows."

Pathline Identification Number \{ integer \},

Start Time \{ real \},

Units $[s|\min | \mathrm{h} \mid$ day $|\mathrm{wk}| \mathrm{yr}]$,

Initial $x$ - or $r$-Direction Coordinate \{ real \},

Units $[\mathrm{m} / \mathrm{ft} / \mathrm{cm}]$,

Initial $y$ - or $\theta$-Direction Coordinate \{ real \},

Units $[\mathrm{m}|\mathrm{ft}| \mathrm{cm}]$,

Initial z-Direction Coordinate $\{$ real \}, Units $[\mathrm{m} / \mathrm{ft} / \mathrm{cm}]$,

Termination Condition [ Boundary | Saturation $|X| Y \mid Z$ ],

Termination-Plane Index $\{$ integer $\}$,

\section{Comments}

The pathline identification number is purely for differentiating between pathlines. The identification numbers do not need to be in strictly increasing order or follow other restrictions.

The boundary termination condition directs MSTS to trace a pathline until the pathline encounters a problem domain boundary (including boundaries represented by internal nodes) or the end of the simulation. This is the default termination condition.

A saturation termination condition may be used only for pathlines that begin in an unsaturated region. The pathline is traced until fully saturated conditions are encountered, a grid boundary is reached, or the simulation ends.

The termination-plane conditions (X, $\mathrm{Y}$, and $\mathrm{Z}$ ) are used to force MSTS to stop tracing a pathline when a particular $X, Y$, or $Z$ plane is encountered and record the travel time. If this condition is used, the value of the index of the surface plane must also be provided. The termination-plane associated with a particular node index is defined for the lower cell surface (e.g., west, south, bottom). 


\section{Examples}

Here three pathlines are defined for travel time calculations in a two-dimensional $(x-z)$ unsaturated domain problem. All three are initially at the top of the domain $(z=10 \mathrm{~m})$. The first pathline will be traced until a boundary is encountered. The second will be traced until it reaches the water table at the bottom of the problem domain or a boundary. The third will terminate when (and if) it encounters the point half-way down at $z=5 \mathrm{~m}$. All are started at time zero.

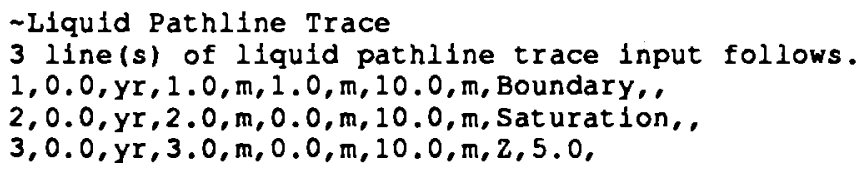




\subsubsection{Output Control Card}

\section{Function}

The Output Control card is used to specify conventional output, plotting output, and reference nodes output. Output controlled through this card is recorded on the "output" file, the "plot" file, and the output screen. Conventional output, which includes input records, simulation process data, reference node data, and field variable data is recorded on the "output" file. Plotting output, which contains condensed formats of field data, is recorded on the "plot" file. Reference nodes output, which includes selected field variables data for a maximum of four nodes is recorded both on the "output" file and output screen. Output times, structures, and formats are also controlled with the Output Control card. The "output" file contains records of specified field variables over a specified domain, presented as two-dimensional arrays of information. Conversely, the "plot" file contains records of specified field variables for every node (including inactive nodes), ordered sequentially by node number. Node numbers are ordered in the sequence row, column, tier, where rows represent east-west directions, columns represent north-south directions, and tiers represent top-bottom directions.

\section{Format}

Line 0 "Output Control"

Line 1 Field Variable Domain ("output" file) I Start \{ integer \}, Field Variable Domain ("output" file) I End [ integer ], Field Variable Domain ("output" file) J Start [ integer ], Field Variable Domain ("output" file) J End ( integer \}, Field Variable Domain ("output" file) K Start \{ integer \}, Field Variable Domain ("output" file) K End \{ integer \}, Plane [ xyplane | yzplane | zxplane | yxplane | zyplane | xzplane ],

Line $2 \quad \mathrm{~N}$ \{ integer \} " line(s) of output control input follows."

Line n Field Variable Output Time ("output" file and "plot" file) \{ real \}, Units $[s|\min | \mathrm{h} \mid$ day $|\mathrm{wk}| \mathrm{yr}$ ],

Next Line Reference Node 1 I-index [ integer ], Reference Node 1 J-index \{ integer \}, Reference Node $1 \mathrm{~K}$-index \{ integer \}, Reference Node 2 I-index [ integer \}, Reference Node 2 J-index \{ integer \}, Reference Node $2 \mathrm{~K}$-index \{ integer \}, Reference Node 3 I-index [ integer \}, Reference Node $3 \mathrm{~J}$-index \{ integer \}, Reference Node $3 \mathrm{~K}$-index \{ integer \}, Reference Node 4 I-index [ integer \}, 
Reference Node 4 J-index \{ integer \}, Reference Node $4 \mathrm{~K}$-index \{ integer $\}$,

Reference Node Output Frequency (Time Steps per Output)\{ integer \},

Next Line $\quad \mathrm{N}\{$ integer \} " line(s) of output control input follows."

Line n ["output" file field variable- see Table 6.3 for acceptable field variable names\},

Next Line $\quad \mathbf{N}\{$ integer \} " line(s) of output control input follows."

Line $n$ ["plot" file field variable- see Table 6.3 for acceptable field variable names\},

Next Line $\quad \mathrm{N}$ ( integer \} " line(s) of output control input follows."

Line n $\quad$ [reference node field variable- see Table 6.3 for acceptable field variable names

\section{Comments}

Because of line width restrictions on computer screens, a maximum of seven field variables may be selected for reference node output. Although there is no limit to the number of field variables that may be selected for "output" file and "plot" file output, the number of selected field variables should be limited to maintain conveniently sized files. The output field velocities associated with a particular node index are defined for the lower surface (e.g., west, south, bottom). For example, an $x$-direction velocity defined at a particular node with $(\mathrm{I}, \mathrm{J}, \mathrm{K})$ indices refers to the $x$ direction velocity at the west surface of the node with the same indices. 
Table 6.3. MSTS Field Variables Available for"Output" File,"Plot" File, and Reference Node Records

\begin{tabular}{|c|c|}
\hline Field Variable & Name Used in Output Control Card \\
\hline$P_{l}$ & Liquid Pressure \\
\hline$P_{g}$ & Gas Pressure \\
\hline$T$ & Temperature \\
\hline$C$ & Species Concentration \\
\hline$s_{\ell}$ & Liquid Saturation \\
\hline$s_{g}$ & Gas Saturation \\
\hline$x_{1}^{a}$ & Liquid Phase Air Mass Fraction \\
\hline$x_{g}^{a}$ & Gas Phase Vapor Mass Fraction \\
\hline$k_{r \ell}$ & Liquid Phase Relative Permeability \\
\hline$k_{r g}$ & Gas Phase Relative Permeability \\
\hline$\rho_{\ell}$ & Liquid Density \\
\hline$\rho_{g}$ & Gas Density \\
\hline$H_{T}$ & Total Hydraulic Head \\
\hline$\theta$ & Volumetric Moisture Content \\
\hline$x^{a}$ & Species Liquid Phase Mole Fraction \\
\hline$x_{g}^{a}$ & Species Gas Phase Mole Fraction \\
\hline$v_{\ell}$ & $\mathrm{X}$-Direction Liquid Velocity \\
\hline$v_{\ell}$ & Y-Direction Liquid Velocity \\
\hline$v_{\ell}$ & Z-Direction Liquid Velocity \\
\hline$V_{g}$ & $\mathrm{X}$-Direction Gas Velocity \\
\hline$V_{g}$ & Y-Direction Gas Velocity \\
\hline$v_{g}$ & Z-Direction Gas Velocity \\
\hline$\dot{Q}_{e, \gamma}$ & X-Direction Heat Transfer Rate \\
\hline
\end{tabular}


Table 6.3. (Contd)

Field Variable Name Used in Output Control Card

$\dot{Q}_{e, \gamma}$

Y-Direction Heat Transfer Rate

$\dot{Q}_{e, \gamma}$

Z-Direction Heat Transfer Rate

$\dot{Q}_{c, \gamma}$

X-Direction Species Flux

$\dot{Q}_{c, \gamma}$

Y-Direction Species Flux

$\dot{Q}_{c, \gamma}$

Z-Direction Species Flux

\section{Examples}

Careful use of the Output Control card will yield output to meet the user's needs without swamping files and computer monitors with undesired information. Here is an example of an Output Control card:

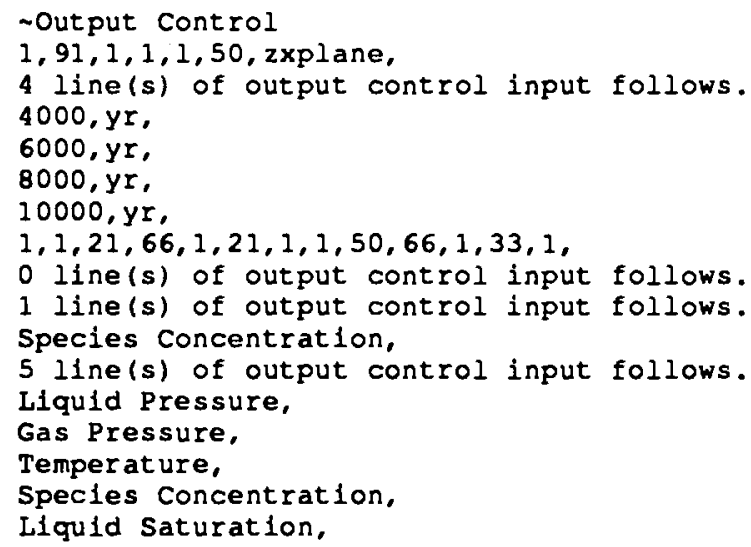

In the example, MSTS output is specified for the region between nodes 1 and 91 in the $x$ - or $r$ direction, nodes 1 and 1 in the $y$-or $\theta$-direction, and nodes 1 and 50 in the $z$-direction. Output is requested at four simulation times: $4000,6000,8000$, and 10,000 years. Four reference nodes are designated at node positions $(1,1,21),(66,1,21),(1,1,50)$, and $(66,1,33)$. No field variables will be output to "output" file. Only species concentration will be recorded to the "plot" file. Five field variables will be displayed on the computer monitor during the simulation for the reference nodes: liquid pressure, gas pressure, temperature, species concentration, and liquid saturation. 


\subsubsection{Surface Flux Integrator Card}

\section{Eunction}

The Surface Flux Integrator card is use to specify two-dimensional planes across which fluxes of liquid mass, gas mass, thermal energy, and species mass are recorded and integrated after each simulation time step. Surface orientation is with respect to an active node.

\section{Format}

Line 0

" Surface Flux Integrator"

Line 1

$\mathrm{N}$ ( integer \} " line(s) of surface flux integrator input follows."

Line $n$

Surface Orientation [ X-Direction: West Surface I X-Direction: East Surface I YDirection: South Surface I Y-Direction: North Surface I Z-Direction: Bottom Surface | Z-Direction: Top Surface ],

Surface Flux Type [ Liquid Phase I Gas Phase I Thermal Energy I Species

I Start \{ integer \}, Concentration ],

I End \{ integer \},

J Start \{ integer \},

$\mathrm{J}$ End \{ integer \},

K Start \{ integer \},

$\mathrm{K}$ End [ integer \},

\section{Comments}

The surface flux integrator serves several purposes. For example, the surface flux integrator feature can be used to test for steady-state conditions by comparing influx and outflow of a quantity at domain boundaries. More commonly, the surface flux integrator is used to determine the flux of a contaminant or the heat transfer across an outflow boundary as a function of simulation time. The surface flux integrator considers the diffusive, advective, and dispersive components of flux. The release of a radionuclide to the water table from an unsaturated model with a saturated lower boundary condition is an example of this use.

\section{Examples}

If no surface flux integration is desired, enter the following for the Surface Flux Integrator card:

\footnotetext{
-Surface Flux Integrator

o line(s) of surface flux integrator input follows.
} 
The following input card could be used to obtain a record of water flux entering and leaving a onedimensional, vertically oriented, 50-node problem in which the water direction is downward:

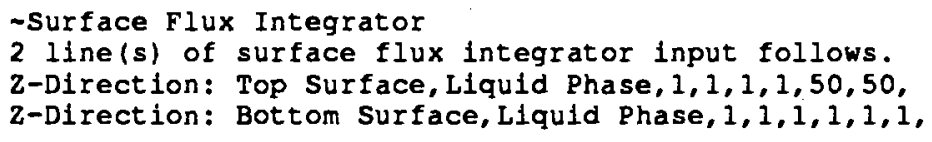




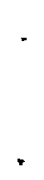




\subsection{Water-Retention and Relative-Permeability Functions}

A number of functional relations are available for use in MSTS to define the water-retention and relative-permeability characteristics of a porous medium. The available functions are defined in this section for reference.

\section{1 van Genuchten Relations}

The van Genuchten (1980) relations are available in MSTS for defining water-retention and liquid relative-permeability functions. For this functional description, effective liquid saturation $\left(s \ell^{*}\right)$ is defined as

$$
s_{\ell}^{*}=\frac{s_{\ell}-s_{\ell r}}{1-s_{\ell r}}
$$

where $s_{\ell}$ is the liquid saturation and $s_{\ell r}$ is the residual liquid saturation, with the restriction $0 \leq s_{\ell r} \leq s_{\ell} \leq 1$. The effective liquid saturation is expressed as a function of tension head $(\psi)$ in the following manner:

$$
\begin{array}{ll}
s_{\ell}^{*}=\left[1+(\alpha \psi)^{n}\right]^{-m} & \psi<0 \\
s_{\ell}^{*}=1 & \psi \geq 0
\end{array}
$$

where $\alpha$ and $n$ are empirically determined van Genuchten fitting parameters ( $\alpha$ has the dimension $\mathrm{L}^{-1}$ and $n$ is dimensionless) and $m$ is a function of $n$ :

$$
m=1-\frac{1}{n}
$$

The equation used to compute liquid relative permeability under the van Genuchten relations depends on whether the formula of Burdine (1953) or Mualem (1976) is chosen. If Burdine's equation is specified, the liquid relative hydraulic conductivity $\left(k_{r \ell}\right)$ under unsaturated conditions is expressed as

$$
k_{r \ell}\left(s_{\ell}^{*}\right)=\left(s_{\ell}^{*}\right) 2\left[1-\left(1-\left(s_{\ell}^{*}\right)^{\frac{1}{m}}\right)^{m}\right]
$$

but if Mualem's equation is specified, then the expression for $k_{r \ell}$ is 


$$
k_{r \ell}\left(s_{\ell}^{*}\right)=\left(s_{\ell}^{*}\right)^{\frac{1}{2}}\left[1-\left(1-\left(s_{\ell}^{*}\right)^{\frac{1}{m}}\right)^{m}\right]^{2}
$$

Section 6.3.6 (Liquid Relative Permeability Card) indicates how to specify Mualem's or Burdine's formula in MSTS input.

\subsection{Brooks and Corey Relations}

The Brooks and Corey relations are available in MSTS for defining water-retention and liquid relative-permeability functions. For this functional description, effective liquid saturation $\left(s \ell^{*}\right)$ is defined as

$$
s_{\ell}^{*}=\frac{s_{\ell}-s_{\ell I}}{1-s_{\ell I}}
$$

where $s_{\ell}$ is the liquid saturation and $s_{\ell r}$ is the residual liquid saturation, with the restriction $0 \leq s_{\ell r} \leq s_{\ell} \leq 1$. The effective liquid saturation is expressed as a function of tension head $(\psi)$ in the following manner.

$$
\begin{array}{ll}
s_{\ell}^{*}=\left(\frac{\psi}{\psi^{*}}\right)^{-m} & \psi<\psi^{*} \\
s_{\ell}^{*}=1 & \psi \geq \psi^{*}
\end{array}
$$

where $\psi^{*}$ is the air-entry head (minimum drainage capillary pressure at which a continuous nonwetting phase exists) and $m$ is an empirical constant. The constant $m$ is a measure of the poresize distribution of the media.

The liquid relative-permeability function depends on whether Burdine (1953) or Mualem (1976) relations are specified. If Burdine relations are chosen, then liquid relative permeability $\left(k_{r} \ell\right)$ is computed from

$$
k_{r \ell}\left(s_{l}^{*}\right)=s_{l}^{*}\left(3+\frac{2}{m}\right)
$$

while if Mualem relations are chosen, the equation used is

$$
k_{r \ell}\left(s_{\ell}^{*}\right)=s_{\ell}^{*}\left(\frac{5}{2}+\frac{2}{m}\right)
$$


Section 6.3.6 (Liquid Relative Permeability Card) indicates how to specify Mualem's or Burdine's formula in MSTS input.

\subsection{Haverkamp Relations}

The Haverkamp relations (Haverkamp et al. 1977) are available in MSTS for defining waterretention and liquid relative-permeability functions. For this functional description, effective liquid saturation $\left(s_{\ell}^{*}\right)$ is defined as

$$
s_{\ell}^{*}=\frac{s_{\ell}-s_{\ell I}}{1-s_{\ell I}}
$$

where $s_{\ell}$ is the liquid saturation and $s_{\ell r}$ is the residual liquid saturation, with the restriction $0 \leq s_{\ell \mathrm{r}} \leq s_{\ell} \leq 1$. The effective liquid saturation is expressed as a function of tension head $(\psi)$ in the following manner.

$$
\begin{array}{ll}
s_{\ell}^{*}=\frac{\alpha}{\alpha+(\ln |100 \psi|)^{\beta}} & \psi<0 \\
s_{\ell=1}^{*} & \psi \geq 0
\end{array}
$$

where $\alpha$ and $\beta$ are curve-fitting parameters and tension head is expressed in meters. The liquid relative permeability $\left(k_{r \ell}\right)$ is computed from

$$
k_{r \ell}=\frac{A}{A+(100 \psi)^{B}}
$$

where $A$ and $B$ are curve-fitting parameters and tension head is again expressed in meters.

\subsection{Corey Relations}

Corey's Curves are available in MSTS for defining water-retention, liquid relative-permeability, and gas relative-permeability functions. For this functional description, effective liquid saturation $\left(s_{\ell}^{*}\right)$ is defined as

$$
s_{\ell}^{*}=\frac{s_{\ell}-s_{\ell r}}{1-s_{\ell r}-s_{s r}}
$$

where $s_{\ell}$ is the liquid saturation, $s_{\ell}$ is the residual liquid saturation, and $s_{s r}$ is the entrapped air 
saturation with the restriction that $\left(s_{\ell r}+s_{s r}\right)<1$. The relative liquid permeability $\left(k_{r \ell}\right)$ is

$$
k_{\mathrm{r} \ell}=\left(s_{\ell}^{*}\right)^{4}
$$

and the relative gas permeability $\left(k_{r g}\right)$ is

$$
k_{r g}=\left(1-s_{\ell}^{*}\right)^{2}\left[1-\left(s_{\ell}^{*}\right)^{2}\right]
$$

\subsection{Fatt and Klikoff Relations}

The relations of Fatt and Klikoff (1959) are available in MSTS for defining water-retention, liquid relative-permeability, and gas relative-permeability functions. For this functional description, effective liquid saturation $\left(s \ell^{*}\right)$ is defined as

$$
s_{\ell}^{*}=\frac{s_{\ell}-s_{\ell r}}{1-s_{\ell r}}
$$

where $s_{\ell}$ is the liquid saturation and $s_{\ell} r$ is the liquid residual saturation with the restriction $0 \leq s_{\ell r} \leq s_{\ell} \leq 1$. Liquid relative permeability $\left(k_{r \ell}\right)$ is computed as

$$
k_{\mathrm{r} \ell}=\left(s_{\ell}^{*}\right)^{3}
$$

and gas relative permeability $\left(k_{r g}\right)$ is expressed as

$$
k_{r g}=\left(1-s_{\ell}^{*}\right)^{3}
$$

\subsection{Leverett Relations}

Leverett's function (Leverett 1941; Udell and Fitch 1985) is available in MSTS for defining water-retention, liquid relative-permeability, and gas relative-permeability functions. For this functional description, effective liquid saturation $\left(s_{\ell}^{*}\right)$ is defined as

$$
s_{\ell}^{*}=\frac{s_{\ell}-s_{\ell \mathrm{I}}}{1-s_{\ell \mathrm{T}}}
$$

with the restriction that $0 \leq s_{\ell r} \leq s_{\ell} \leq 1$. The capillary pressure $\left(P_{c a p}\right)$ is computed from

$$
P_{8}-P_{l}=-P_{\text {cap }}^{*} \sigma^{Q w} f
$$


where $\sigma^{a w}$ is the surface tension of water (supplied internally by MSTS as a function of temperature) and $f$ is computed from

$$
f=1.417\left(1-s_{\ell}^{*}\right)-2.120\left(1-s_{\ell}^{*}\right)^{2}+1.263\left(1-s_{\ell}^{*}\right)^{3}
$$

\subsection{Touma and Vauclin Relations}

Touma and Vauclin (1986) used, for modeling purposes, analytical expressions fitted to experimental data to describe the dependence of liquid and gas relative permeabilities on liquid saturation. The MSTS form of their expression to describe liquid relative permeability as function of volumetric moisture content $\theta$ is

$$
k_{r \ell}=A_{\ell} \theta^{B} \ell^{\prime}
$$

where $A_{\ell}$ and $B_{\ell}$ are empirical fitting parameters. The gas relative permeability is expressed as a function of the capillary pressure head, or tension head $\psi$.

$$
k_{r g}=\frac{A_{g}}{A_{g}+\psi^{B}}
$$

where $A_{g}$ and $B_{g}$ are empirical fitting parameters. This function was introduced to MSTS for the explicit purpose of simulating problems for Section 8.6 (One-Dimensional Two-Phase Infiltration).

\subsection{Dual-Porosity Model With van Genuchten Relations}

An equivalent-continuum (dual-porosity) model is available in MSTS to define water-retention and liquid relative-permeability functions. The model represents a composite domain that includes the effects of both fracture- and matrix-flow regimes. The equivalent bulk aqueous saturation $\left(s_{l_{b}}\right)$ is approximated as

$$
s_{\ell_{b}}=\frac{s_{\ell_{f}} n_{T_{f}}+s_{\ell_{m}}\left(1-n_{T_{f}}\right) n_{T_{m}}}{n_{T_{f}}+\left(1-n_{T_{f}}\right) n_{T_{m}}}
$$

where $s_{\ell_{f}}$ is the liquid saturation of the fractures, $s_{\ell_{m}}$ is the liquid saturation of the matrix, $n_{T}$ is the total porosity of the fractures (ratio of the fracture volume to the total bulk volume), and $n_{T_{m}}$ is 
the total porosity of the matrix (ratio of the volume of the voids in the matrix to the total bulk volume). The equivalent bulk aqueous relative permeability $\left(k_{r \ell_{b}}\right)$ is defined as

$$
k_{r \ell_{b}}=k_{r \ell_{m}}\left(1-n_{T s}\right)+k_{r \ell_{s}}{ }^{n} T_{s}
$$

where $k_{r \ell}$ and $k_{r \ell}$ are the fracture and matrix relative liquid permeabilities, respectively. 


\subsection{Sample Problems}

Eight sample problems are presented in this section. The sample problems were selected to demonstrate the application of MSTS to a variety of thermal and hydrogeologic transport problems. The eight sample problems are summarized in Table 8.1 and include single-phase flow and transport, multiphase flow and heat transfer, interphase mass transfer, and phase transition. The sample problems demonstrate the application of the code's various solution modes and provide complete examples of MSTS input files. Sample problem results are compared to analytical, semianalytical, independent numerical, and experimental results.

In all of these sample problems, the input files for the MSTS code used to direct the simulations described in each section (and in some cases the output files) are shown as "exhibits." Each exhibit is a verbatim copy of an input or output file provided to assist in understanding how the input file is prepared for a specific problem and to show complete file examples. Line numbers are provided on the left side of each exhibit; these are not a part of the actual file. The line numbers are available only to aid in reading the input file and referencing portions of it in the discussion. When the line that appears in an input file is too wide to display in this document, the continuation is shown indented on the subsequent line without a line number.

Where comparisons are made to analytical or semi-analytical results, the relative root mean square $(R R M S)$ was adopted as the performance indicator. This statistic is computed as

$$
R R M S=\sqrt{\frac{\sum_{i=1}^{k}\left(\frac{\alpha_{i}-n_{i}}{\alpha_{i}}\right)^{2}}{k}}
$$

where $\alpha_{i}=$ analytic solution parameter value (e.g., liquid saturation) at point i, $n_{i}=$ numerical solution parameter value (liquid saturation) at point $i$, and $k=$ number of comparison points. To judge the accuracy level of this verification test, the performance categories set by Baca and Magnuson (1990) are adopted. These ranges are "somewhat arbitrary but nevertheless represent a consistent basis for evaluating the quality of the comparison" (Baca and Magnuson 1990). The ranges are: 
- Excellent - $\quad R R M S \leq 0.01$

- Good- $\quad$ RRMS $\leq 0.05$

- Acceptable- $\quad R R M S \leq 0.10$

- Unacceptable- $\quad R R M S>0.10$

Table 8.1. Summary of Sample Problems

\section{Problem Description}

1 Single-Node Evaporation

2 Single-Node Condensation

3 Hot Two-Phase Flow

4 Philip's Vertical Infiltration

5 Two-Dimensional Infiltration

6 Two-Phase Infiltration

7 One-Dimensional Heat Pipe

8 Unsaturated Transport
Dimensions

1

1

Energy

1

1

2

Water
Water-Air-Energy
Water

Energy

phase transitions, component appearance and disappearance, mass conservation

phase transitions, component appearance and disappearance, mass conservation

energy conservation

code verification against semi-analytic solution (Philip 1957)

code comparison to numerical results (Pruess 1987)

Water, Water-Air

1 Water-Air-Energy

code verification against known semi-analytical solution (Udell and Fitch 1985)

air-flow effects on water infiltration; code comparison to experimental and numerical results (Touma and Vauclin 1986)

code verification against semi-analytical solution (Ross et al. 1982; Smiles et al. 1977) 


\subsection{Single-Node Evaporation}

The single-node-evaporation problem involves the evaporation of liquid water within a closed adiabatic volume of partially saturated porous medium. The evaporation process occurs through the addition of heat into the control volume. Sufficient heat is added to the system to desaturate the porous medium. Because the control volume surfaces are closed and adiabatic, the initial quantities of air and water mass should remain constant throughout the simulation. Upon desaturation of a node, MSTS switches the primary variable for the water mass conservation equation from the liquid pressure to the water-vapor mass fraction. This problem demonstrates this numerical transition from two-phase conditions to gas-phase-only conditions. The problem requires the coupled solution of the water mass, air mass, and energy conservation equations.

\subsubsection{Problem Domain and Input Parameters}

This problem involves a $10-\mathrm{m}^{3}$ cube of porous media, with a total porosity of 0.50 , rock density of $2650 \mathrm{~kg} / \mathrm{m}^{3}$, and specific heat of $1000 \mathrm{~J} / \mathrm{kg} \mathrm{K}$. The initial gas pressure, liquid saturation, and temperature are $10^{5} \mathrm{~Pa}$ (absolute), 0.01 , and $90^{\circ} \mathrm{C}$, respectively. A constant internal heat source of $2 \mathrm{MW}$ was applied. All boundary surfaces are adiabatic, no-flow surfaces. A complete description of the problem specifics is given in the "input" file shown in Exhibit 8.1.

\subsubsection{Simulation Results}

Simulation results in terms of the system temperature and liquid saturation are shown in Figure 8.1. The system begins at an initially fully saturated state, and liquid saturation declines with time to a completely desaturated state as water undergoes phase change with increasing temperature. The simulation is halted at 1875 seconds at a final temperature of $363.9^{\circ} \mathrm{C}$, which is slightly below the critical temperature for water. The output file generated by MSTS for the single-nodeevaporation problem is shown in Exhibit 8.2.

\subsubsection{Mass Balance Check}

This problem concerns a closed system in which mass must be conserved. To test that MSTS conserves mass in simulating a phase change, a mass balance check was performed. The check compares the total mass of water in the cube represented by the single node at the initial conditions to the total mass of water at 1875 seconds, the end of the simulation time. The total mass of water in the node $\left(m_{T}^{w}\right)$ is the sum of the water in the aqueous $\left(m_{l}^{w}\right)$ and vapor $\left(m_{g}^{w)}\right.$ phases. 


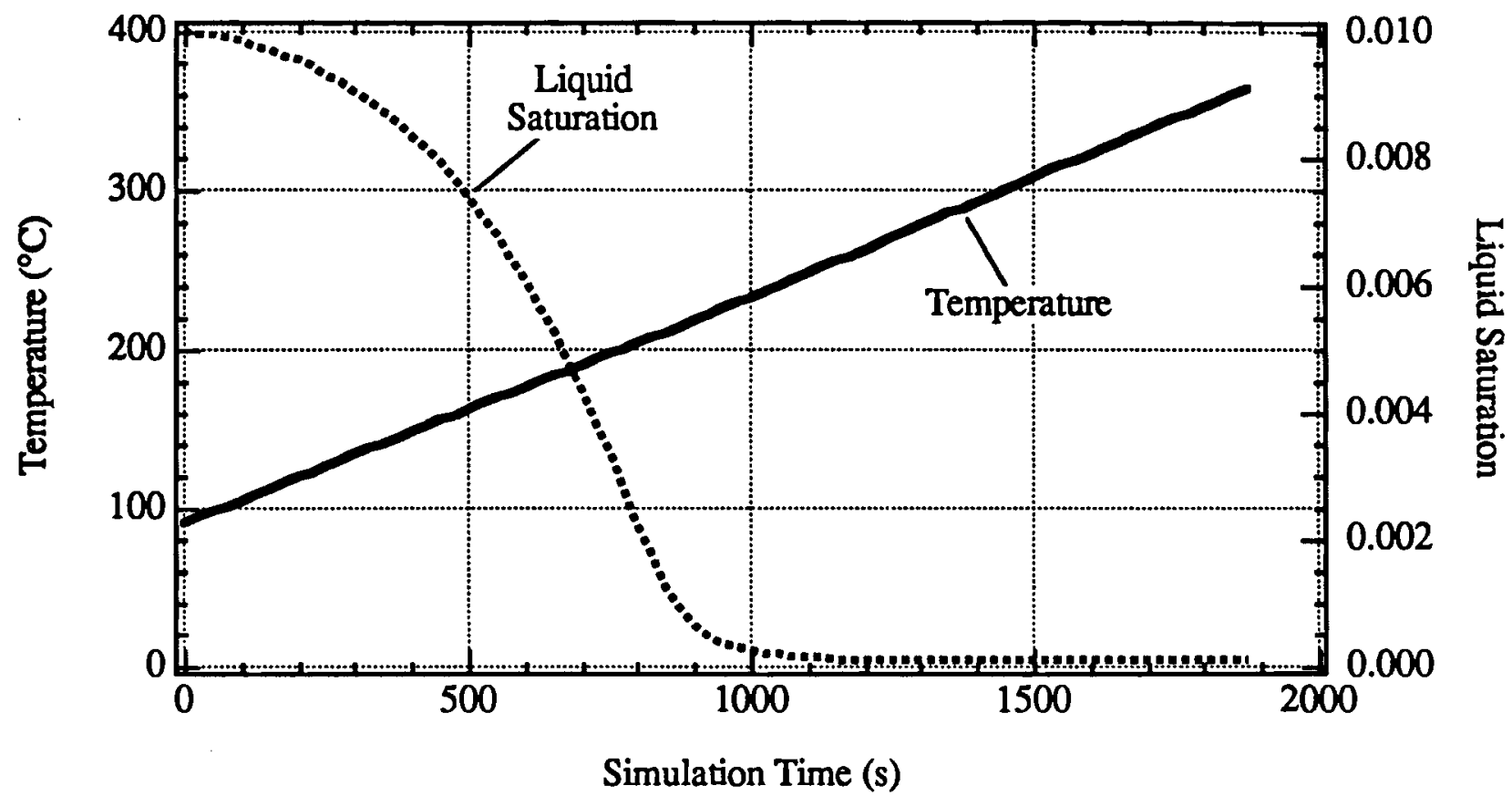

Figure 8.1. MSTS-Predicted Temperature and Liquid Saturation Variation with Time for the Single-Node-Evaporation Problem

The mass of water in the aqueous phase is given by

$$
m_{\ell}^{w}=\eta_{D} V \rho_{\ell}=\left(n_{T}-n_{D}+s_{\ell} n_{D}\right) V \rho_{\ell}
$$

and the mass of water in the gas phase by

$$
m_{g}^{w}=\left(1-s_{\ell}\right) \mathrm{n}_{D} V x_{\ell}^{w} \rho_{g}
$$

In mathematical terms, the total mass in the domain is the sum of the water in each phase:

$$
m_{T}^{w}=m_{\ell}^{w}+m_{g}^{w}
$$

The initial conditions were derived from the problem definition and the final conditions were obtained from the plot file generated by the MSTS code; both are shown in Table 8.2. Substituting the values from Table 8.2 into Equation (8.2), the total water mass in the aqueous phase at time zero (initial) is

$$
\begin{aligned}
m_{\ell}^{w} & =\left(n_{T}-n_{D}+s_{\ell} n_{D}\right) \vee \rho_{\ell} \\
& =[0.50-0.50+(0.01)(0.50)]\left(10 \mathrm{~m}^{3}\right)\left(965.1 \mathrm{~kg} / \mathrm{m}^{3}\right) \\
& =48.255 \mathrm{~kg}
\end{aligned}
$$


Table 8.2. Single-Node-Evaporation Problem Initial and Final Conditions

$\begin{array}{lccc}\text { Variable } & \text { Units } & \text { Initial Conditions } & \text { Final Conditions } \\ V & \mathrm{~m}^{3} & 10.0 & 10.0 \\ n_{T} & - & 0.50 & 0.50 \\ n_{D} & - & 0.50 & 0.50 \\ T & { }^{\circ} \mathrm{C} & 90.0 & 363.86 \\ s_{\ell} & - & 0.01 & 9.6219 \times 10-5 \\ \rho_{\ell} & \mathrm{kg} / \mathrm{m}^{3} & 965.1 & 473.72 \\ \rho_{\&} & \mathrm{~kg} / \mathrm{m}^{3} & 0.7102 & 10.307 \\ x_{\ell}^{w} & - & 0.5964 & 0.97170\end{array}$

Substituting values from Table 8.2 into Equation (8.3), the water mass in the gas phase at time zero is

$$
\begin{aligned}
m_{g}^{w} & =\left(1-s_{\ell}\right) n_{D} V x_{g}^{w} \rho_{g} \\
& =(1-0.01)(0.50)\left(10 \mathrm{~m}^{3}\right)(0.5964)\left(0.7102 \mathrm{~kg} / \mathrm{m}^{3}\right) \\
& =2.09664 \mathrm{~kg}
\end{aligned}
$$

Therefore, the total water mass in the node at time zero is $50.352 \mathrm{~kg}$. When the simulation is completed, the temperature is $363.9^{\circ} \mathrm{C}$ and the water mass in the aqueous phase is

$$
\begin{aligned}
m_{\ell}^{w} & =\left(n_{T}-n_{D}+s_{\ell} n_{D}\right) V \rho_{\ell} \\
& =\left[0.50-0.50+\left(9.6219 \times 10^{-5}\right)(0.50)\right]\left(10 \mathrm{~m}^{3}\right)\left(473.72 \mathrm{~kg} / \mathrm{m}^{3}\right) \\
& =0.2279 \mathrm{~kg}
\end{aligned}
$$

(i.e., there is essentially no water mass in the aqueous phase) and the water mass in the gas phase is

$$
\begin{aligned}
m_{g}^{w} & =\left(1-s_{\ell}\right) n_{D} V x_{g}^{w} \rho_{g} \\
& =\left(1-9.6219 \times 10^{-5}\right)(0.50)\left(10 \mathrm{~m}^{3}\right)(0.97170)\left(10.307 \mathrm{~kg} / \mathrm{m}^{3}\right) \\
& =50.0717 \mathrm{~kg}
\end{aligned}
$$


These two values sum to a total water mass in the node at final conditions of $50.300 \mathrm{~kg}$. The mass difference between initial and final conditions is therefore $50.352-50.300=0.052 \mathrm{~kg}$, or $0.10 \%$. 


\section{Exhibit 8.1. MSTS Input File for Single-Node-Evaporation Problem}

\section{Line No. Input File}

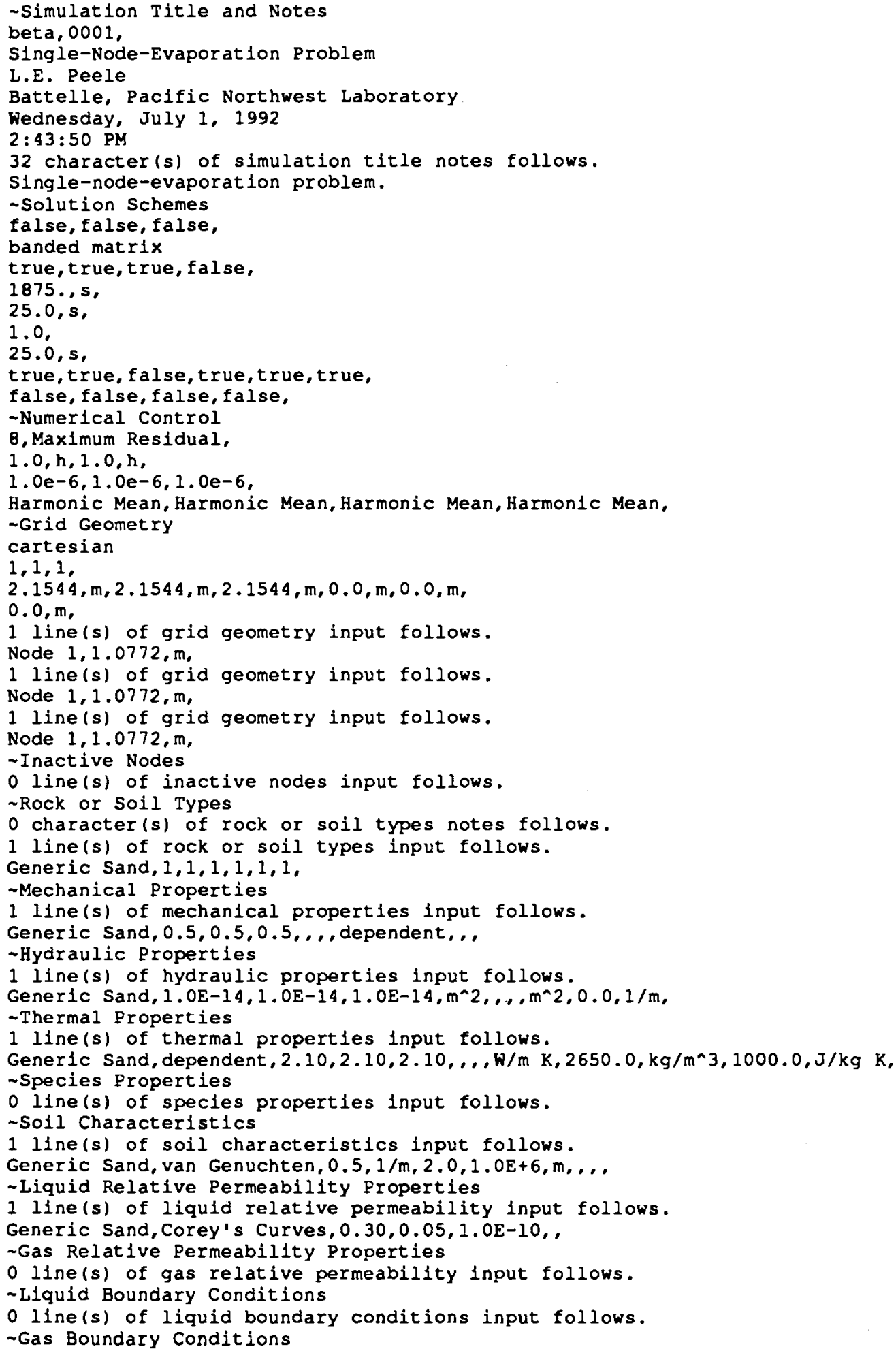




\section{Exhibit 8.1. (Contd)}

\section{Line No Input File}

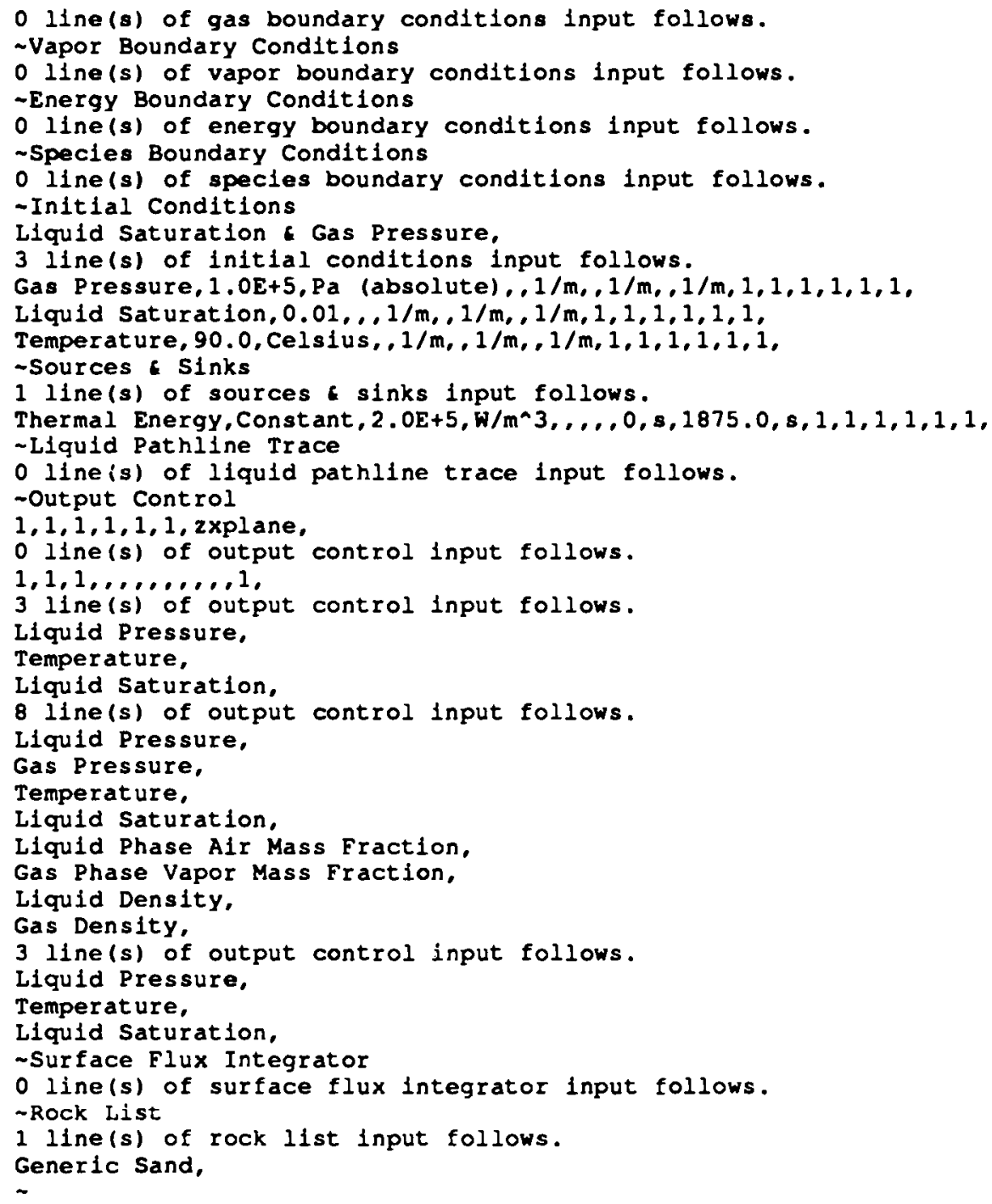


Exhibit 8.2. MSTS Output File for Single-Node-Evaporation Problem

\section{Line No Output File}

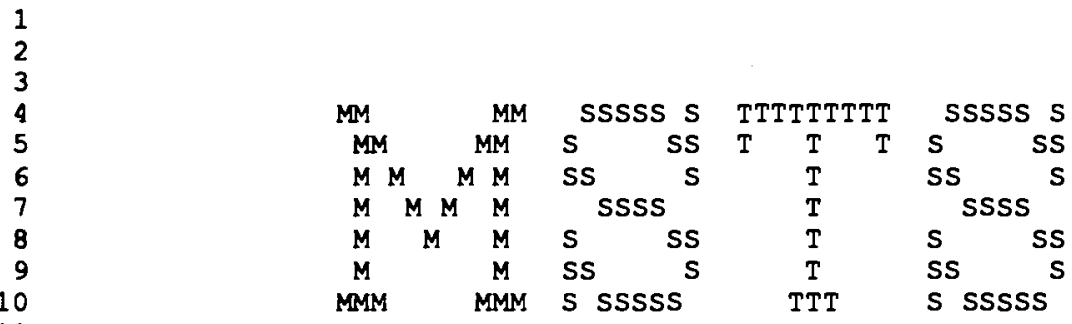

THIS OUTPUT FILE WAS PRODUCED BY THE NUMERICAL MODEL

M S T S

MULTIPHASE SUBSURFACE TRANSPORT SIMULATOR

DEVELOPED BY BATTELLE, PACIFIC NORTHWEST LABORATORY

THIS COMPUTER CODE WAS DEVELOPED UNDER CONTRACT TO

THE UNITED STATES DEPARTMENT OF ENERGY

FOR ENQUIRIES OR ASSISTANCE: CALL 509 375-2183 USA

OR $509 \quad 376-8315$ USA

VERSION: beta REVISION: 0001

EXECUTION DATE: $12 / 7 / 1992$

EXECUTION TIME: $15: 45: 5$

EXECUTION IDENTIFICATION : 127921545

RESULTS FROM THIS VERSION OF MSTS SHOULD NOT BE USED FOR LICENSE RELATED APPLICATIONS.

RECORD OF INPUT DATA

RECORD OF INPUT DATA

Simulation Title and Notes

Simulation Title: Single-Node-Evaporation Problem

User Name: L.E. Peele

Company Name: Battelle, Paclfic Northwest Laboratory

Input Creation Date: Wednesday, July 1, 1992

Input Creation Time: $2: 43: 50 \mathrm{PM}$ 
Exhibit 8.2. (Contd)

\section{Line No Qutput File}

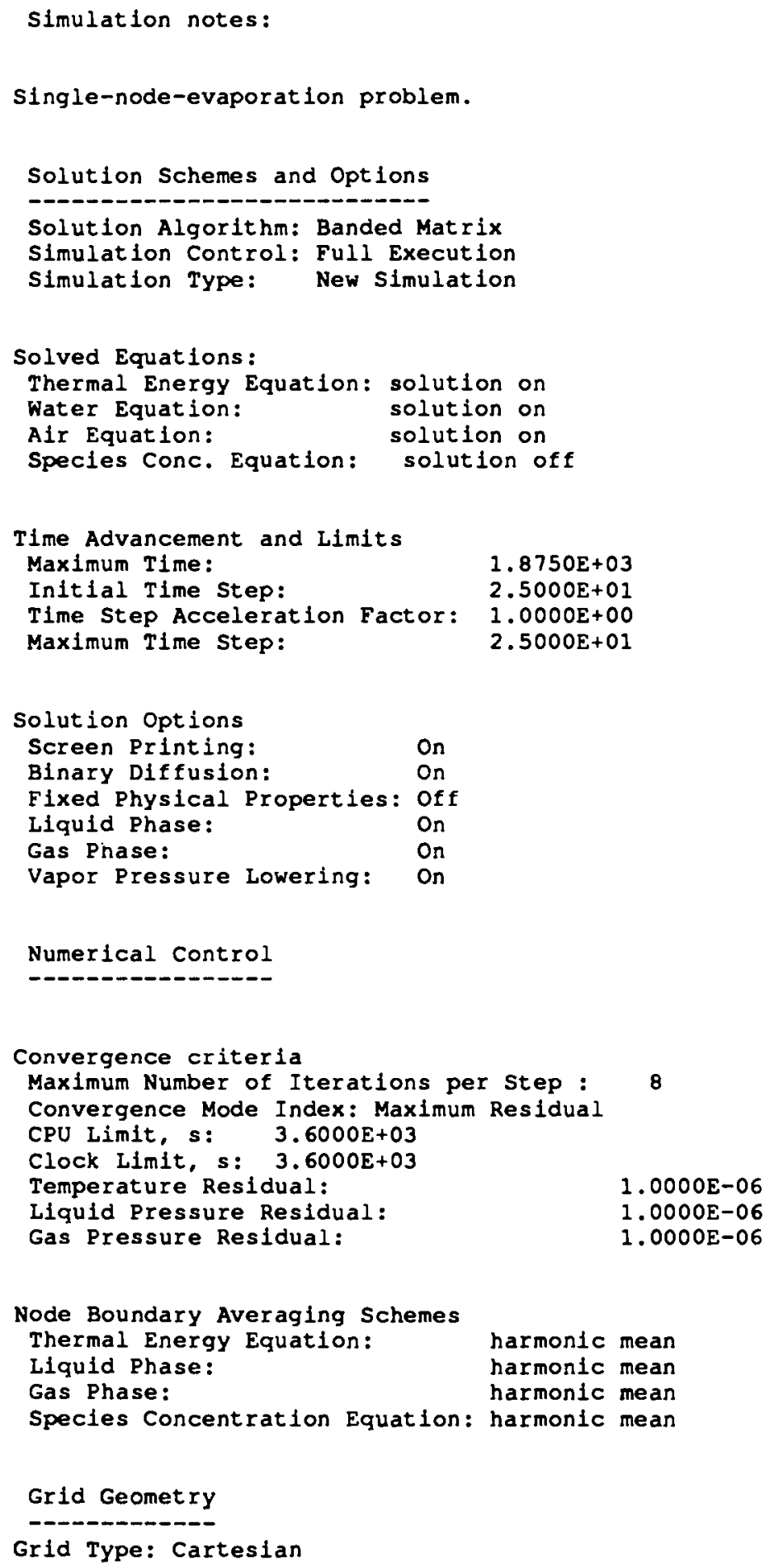




\section{Exhibit 8.2. (Contd)}

\section{Line No Qutput File}

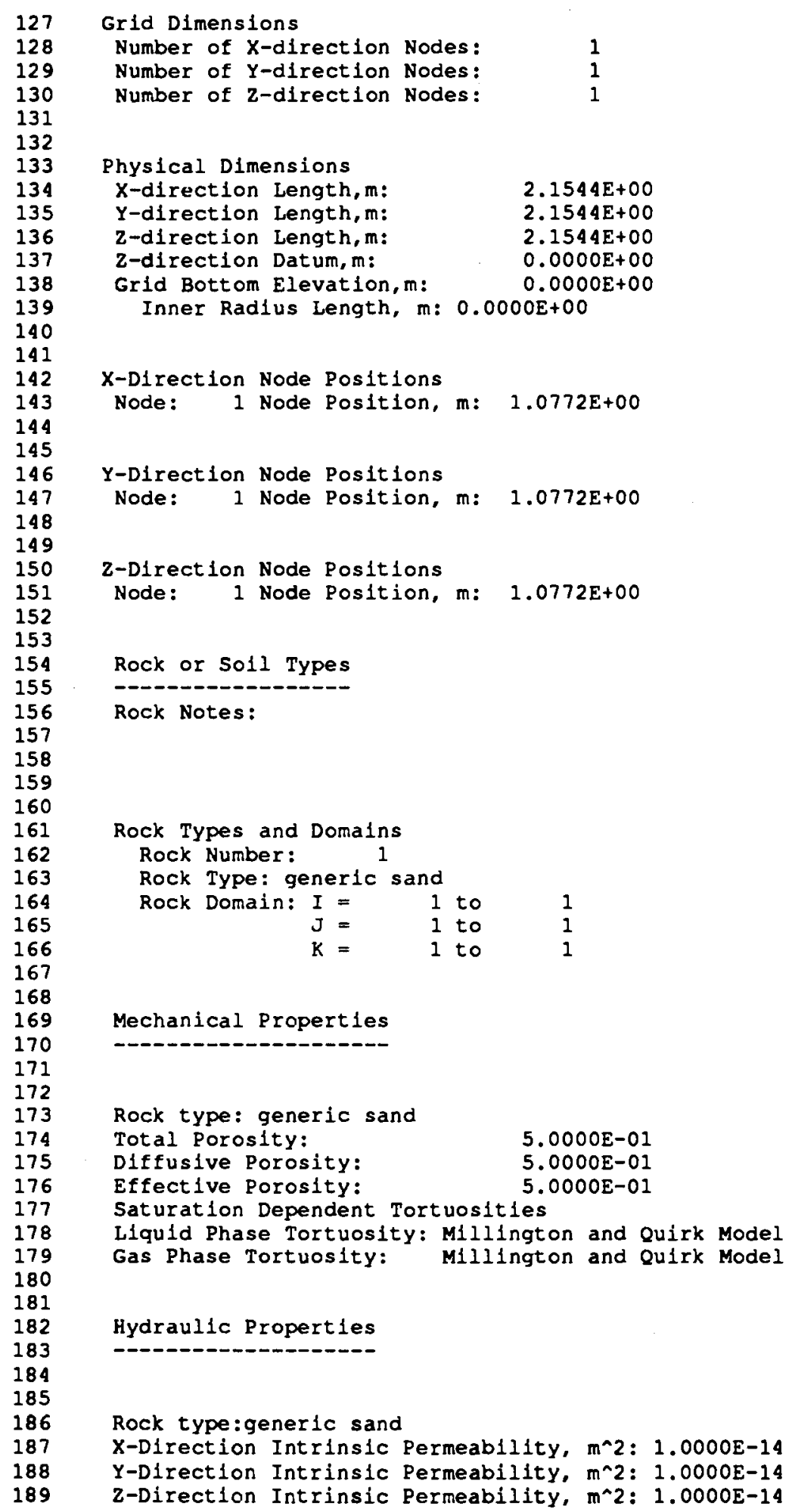

\subsection{1}




\section{Exhibit 8.2. (Contd)}

\section{Line No Output File}

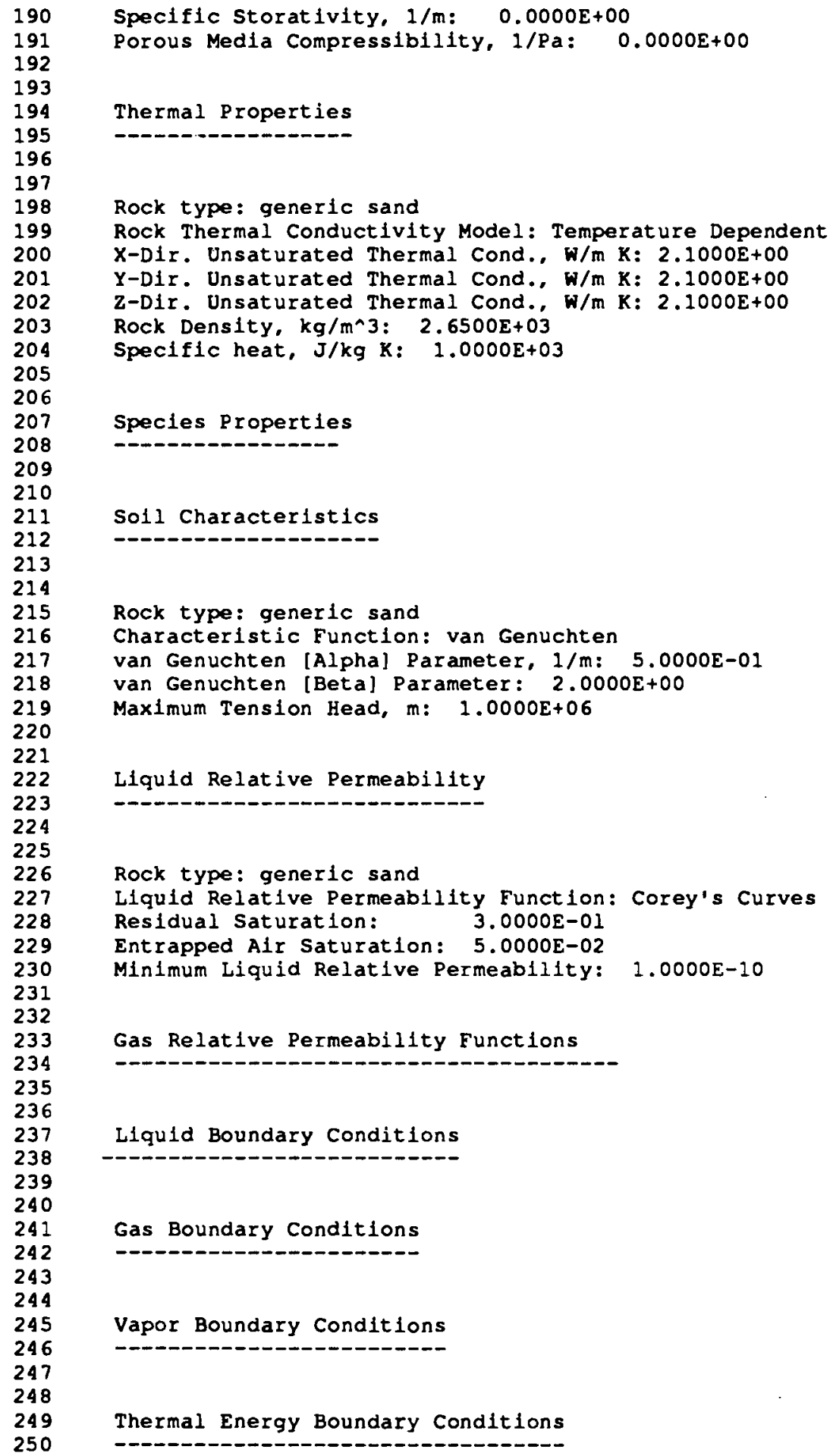


Exhibit 8.2. (Contd)

\section{Line No. Output File}

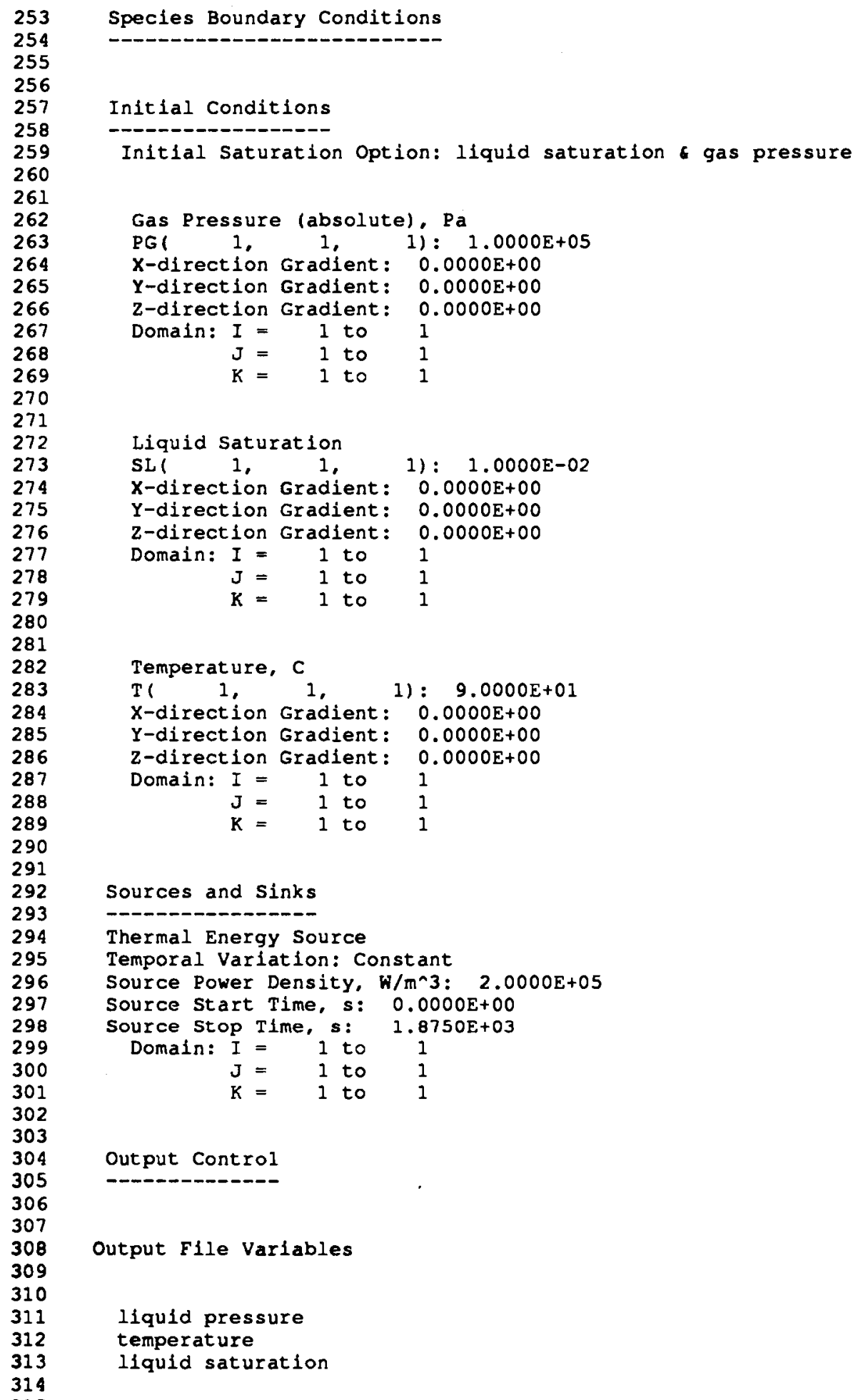




\section{Exhibit 8.2. (Contd)}

Line No Qutput File

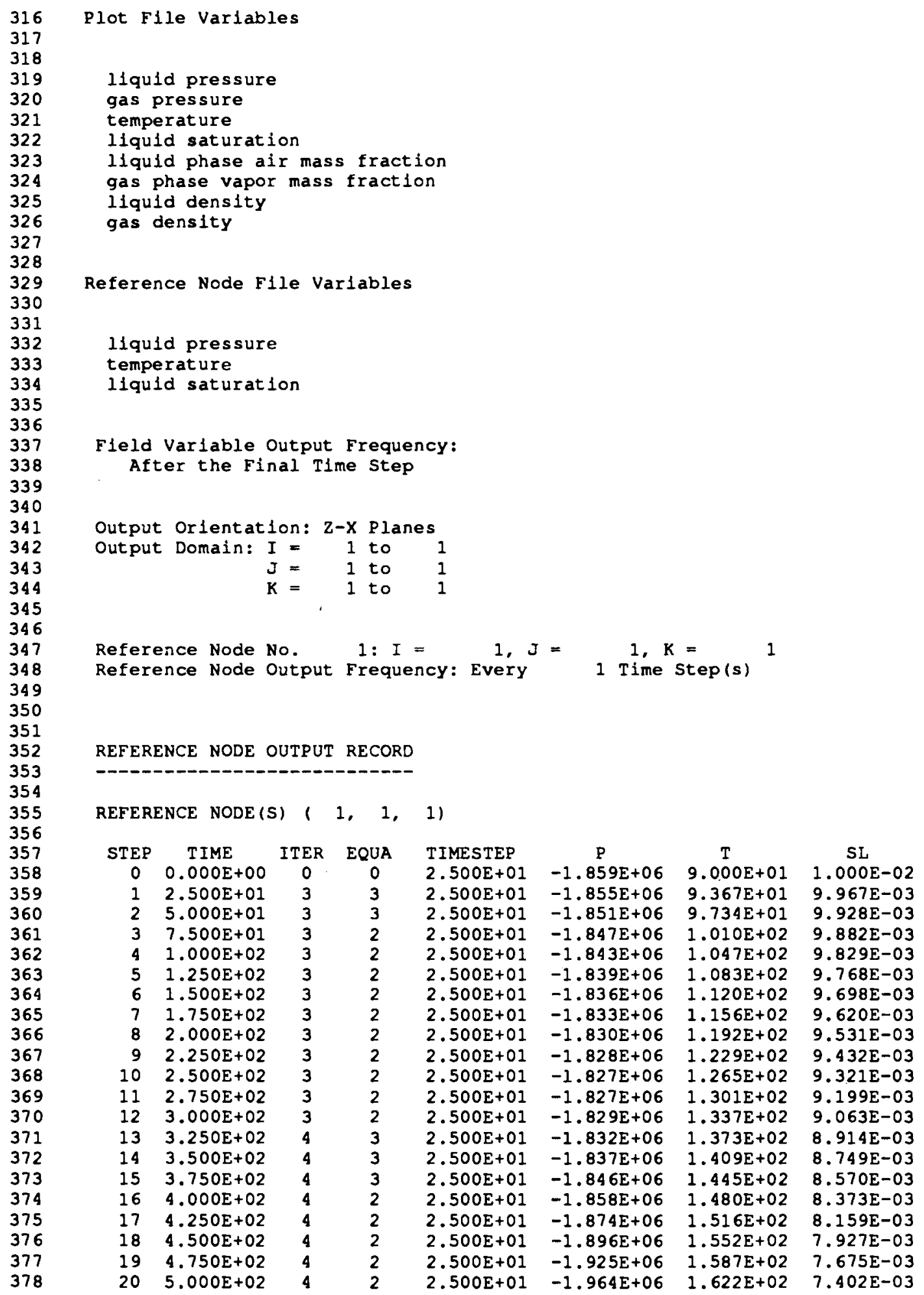


Exhibit 8.2. (Contd)

Line No. Qutput File

$\begin{array}{ccccc}379 & 21 & 5.250 E+02 & 4 & 2 \\ 380 & 22 & 5.50 O E+02 & 4 & 2 \\ 381 & 23 & 5.750 E+02 & 4 & 2 \\ 382 & & & & \\ 383 & \text { REFERENCE NODE (S) } & 1 & 1, & 1, \\ 384 & & & & \\ 385 & \text { STEP } & \text { TIME } & \text { ITER } & \text { EQUA } \\ 386 & 24 & 6.000 E+02 & 4 & 2 \\ 387 & 25 & 6.250 E+02 & 4 & 2 \\ 388 & 26 & 6.500 E+02 & 4 & 2 \\ 389 & 27 & 6.750 E+02 & 4 & 2 \\ 390 & 28 & 7.000 E+02 & 4 & 2 \\ 391 & 29 & 7.250 E+02 & 4 & 2 \\ 392 & 30 & 7.500 E+02 & 4 & 2 \\ 393 & 31 & 7.750 E+02 & 4 & 2 \\ 394 & 32 & 8.000 E+02 & 5 & 2 \\ 395 & 33 & 8.250 E+02 & 5 & 2 \\ 396 & 34 & 8.500 E+02 & 5 & 2 \\ 397 & 35 & 8.750 E+02 & 5 & 2 \\ 398 & 36 & 9.000 E+02 & 4 & 2 \\ 399 & 37 & 9.250 E+02 & 4 & 2 \\ 400 & 38 & 9.500 E+02 & 4 & 2 \\ 401 & 39 & 9.750 E+02 & 4 & 2 \\ 402 & 40 & 1.000 E+03 & 4 & 2 \\ 403 & 41 & 1.025 E+03 & 3 & 2 \\ 404 & 42 & 1.050 E+03 & 3 & 2 \\ 405 & 43 & 1.075 E+03 & 3 & 2 \\ 406 & 44 & 1.100 E+03 & 3 & 2 \\ 407 & 45 & 1.125 E+03 & 3 & 2 \\ 408 & 46 & 1.150 E+03 & 3 & 2 \\ 409 & 47 & 1.175 E+03 & 3 & 2 \\ 410 & & & & \end{array}$
$2.500 E+01-2.014 E+06$
$1.658 \mathrm{E}+02$
$2.500 E+01 \quad-2.080 E+06 \quad 1.693 E+02$
$7.108 \mathrm{E}-03$
$2.500 \mathrm{E}+01$
$1.728 \mathrm{E}+02$
$6.791 \mathrm{E}-03$
$6.450 \mathrm{E}-03$

1)

REFERENCE NODE(S) ( 1, 1, 1)

412

413

414

415

416

417

418

419

420

421

422

423

424

425

426

427

428

429

430

431

432

433

434

435

436

437

438

439

440

STEP TIME ITER EQUA

$48 \quad 1.2005+03$

$49 \quad 1.225 \mathrm{E}+03$

3

2
2
3

$50 \quad 1.250 E+03$

$51 \quad 1.275 \mathrm{E}+03$

TIMESTEP

2.500E+01

2. $500 E+01$

2. $500 E+01$

2. $500 E+01$

2.500E+01

2. $500 E+01$

2. $500 E+01$

2.500E+O1

2. $500 E+01$

2. $500 \mathrm{E}+01$

2. $500 \mathrm{E}+01$

2. $500 E+01$

2.500E+01

2.500E+01

2. $500 E+01$

$2.500 E+01$

2. $500 E+01$

2. $500 E+01$

2.500E+01

2. $500 E+01$

2. $500 E+01$

2. $500 E+01$

$2.500 E+01$

$2.500 E+01$

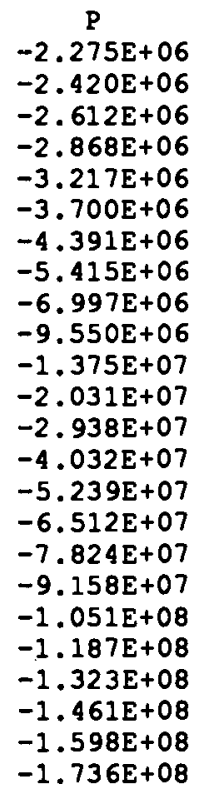

$T$

$1.763 \mathrm{E}+02$

$1.798 \mathrm{E}+02$

$1.832 \mathrm{E}+02$

$1.867 \mathrm{E}+02$

$1.901 \mathrm{E}+02$

$1.936 \mathrm{E}+02$

$1.970 \mathrm{E}+02$

$2.005 E+02$

$2.039 E+02$

$2.074 E+02$

$2.109 E+02$

$2.144 \mathrm{E}+02$

$2.180 \mathrm{E}+02$

$2.217 E+02$

2.254 E+02

$2.291 E+02$

2. $328 \mathrm{E}+02$

$2.365 \mathrm{E}+02$

$2.402 \mathrm{E}+02$

$2.440 \mathrm{E}+02$

$2.477 \mathrm{E}+02$

$2.515 E+02$

$2.552 \mathrm{E}+02$

2. $589 \mathrm{E}+02$

SI

$6.085 \mathrm{E}-03$

5. 695E-03

5.278E-03

$4.834 \mathrm{E}-03$

4. $363 \mathrm{E}-03$

3. 867E-03

$3.347 \mathrm{E}-03$

$2.808 \mathrm{E}-03$

$2.262 \mathrm{E}-03$

$1.732 \mathrm{E}-03$

$1.256 \mathrm{E}-03$

8.807E-04

$6.245 E-04$

$4.625 \mathrm{E}-04$

3. $596 \mathrm{E}-04$

$2.914 \mathrm{E}-04$

2. $437 \mathrm{E}-04$

$2.090 \mathrm{E}-04$

$1.826 \mathrm{E}-04$

$1.620 \mathrm{E}-04$

1. $455 \mathrm{E}-04$

1. $321 \mathrm{E}-04$

$1.208 \mathrm{E}-04$

$1.114 E-04$

1)

TIMESTEP

2.500E+01

2. $500 E+01$

2. $500 E+01$

2. $500 \mathrm{E}+01$

2. $500 E+01$

$2.500 E+01$

2. $500 E+01$

2.500E+01

2. $500 \mathrm{E}+01$

2. $500 \mathrm{E}+01$

2. $500 E+01$

2. $500 \mathrm{E}+01$

$2.500 E+01$

2. $500 E+01$

2. $500 E+01$

2. $500 E+01$

2. $500 E+01$

2. $500 E+01$

2. $500 E+01$

2. $500 \mathrm{E}+01$

2. $500 E+01$

2. $500 E+01$

2. $500 E+01$

$2.500 E+01$

$P$
$-1.874 E+08$
$-2.012 E+08$
$-2.012 E+08$
$-2.012 E+08$
$-2.012 E+08$
$-2.011 E+08$
$-2.011 E+08$
$-2.011 E+08$
$-2.011 E+08$
$-2.011 E+08$
$-2.010 E+08$
$-2.010 E+08$
$-2.010 E+08$
$-2.010 E+08$
$-2.010 E+08$
$-2.009 E+08$
$-2.009 E+08$
$-2.009 E+08$
$-2.009 E+08$
$-2.009 E+08$
$-2.008 E+08$
$-2.008 E+08$
$-2.008 E+08$
$-2.008 E+08$

$\mathrm{T}$

2. $627 \mathrm{E}+02$

$2.664 \mathrm{E}+02$

2. $702 \mathrm{E}+02$

$2.739 E+02$

$2.777 \mathrm{E}+02$

$2.814 \mathrm{E}+02$

$2.852 \mathrm{E}+02$

$2.889 \mathrm{E}+02$

2. $927 \mathrm{E}+02$

2. $964 \mathrm{E}+02$

3. $002 \mathrm{E}+02$

$3.039 \mathrm{E}+02$

$3.076 \mathrm{E}+02$

3. $114 \mathrm{E}+02$

3.151E+02

3. $189 E+02$

$3.226 \mathrm{E}+02$

3. $264 \mathrm{E}+02$

3. $301 \mathrm{E}+02$

3. $339 E+02$

3. $376 E+02$

$3.414 \mathrm{E}+02$

$3.451 \mathrm{E}+02$

$3.489 E+02$

SL

1. $032 E-04$

9. $622 \mathrm{E}-05$

$9.622 \mathrm{E}-05$

$9.622 \mathrm{E}-05$

9. $622 \mathrm{E}-05$

9.622E-05

9. $622 \mathrm{E}-05$

$9.622 \mathrm{E}-05$

9. $622 \mathrm{E}-05$

9. $622 \mathrm{E}-05$

9. $622 \mathrm{E}-05$

9. $622 \mathrm{E}-05$

$9.622 \mathrm{E}-05$

$9.622 \mathrm{E}-05$

9.622 E-05

$9.622 \mathrm{E}-05$

9.622 E-05

$9.622 E-05$

9.622E-05

9.622E-05

9. $622 \mathrm{E}-05$

$9.622 \mathrm{E}-05$

9. $622 \mathrm{E}-0.5$

9. $622 \mathrm{E}-05$

REFERENCE NODE(S) ( 1, 1, 1)

STEP TIME ITER EQUA TIMESTEP

P

$\mathbf{T}$

SI 
Exhibit 8.2. (Contd)

Line No Output File

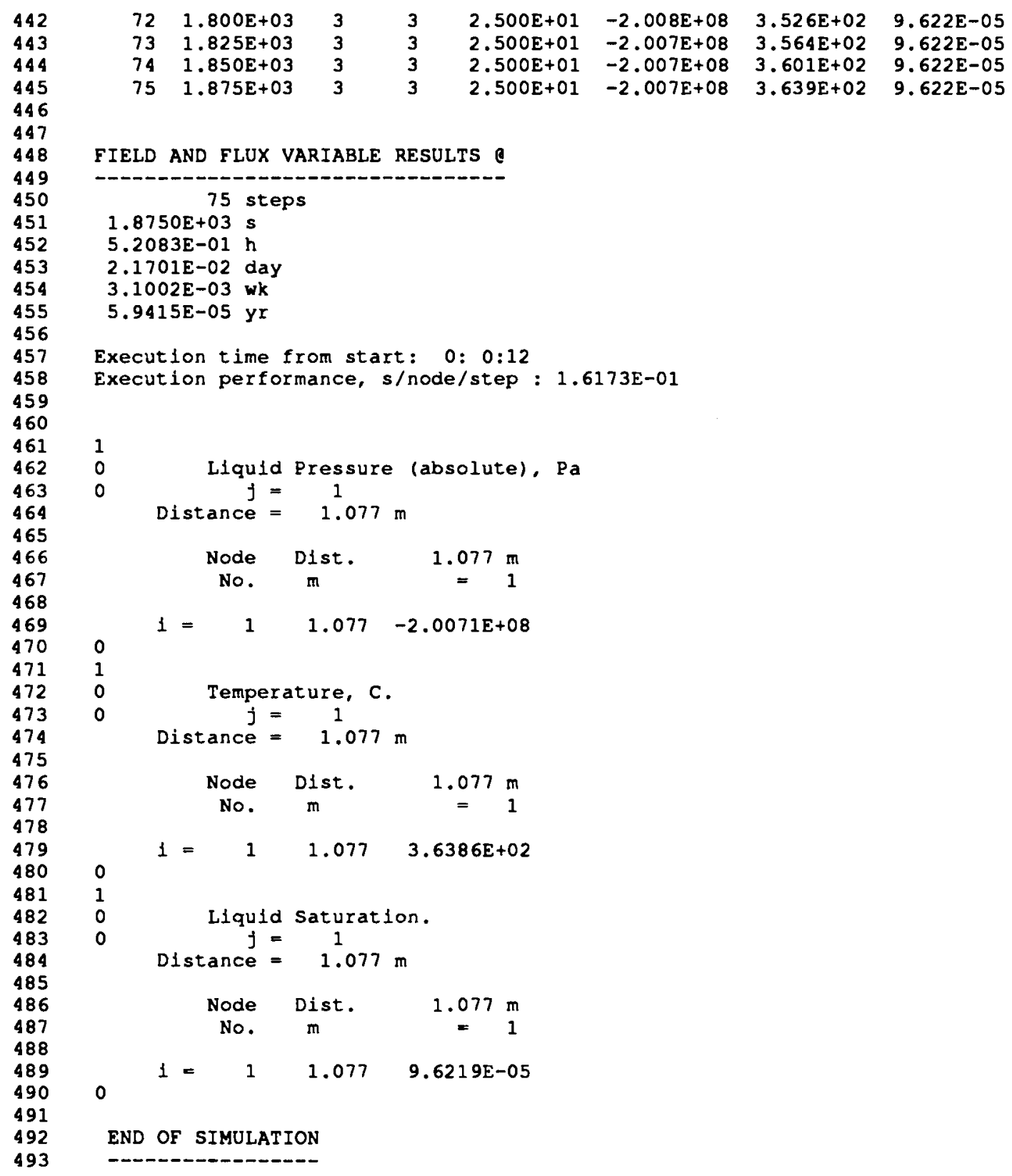




\subsection{Single-Node Condensation}

The single-node-condensation problem involves the condensation of water vapor within a closed adiabatic volume of porous medium initially desaturated. The condensation process occurs through the removal of heat from the control volume. Sufficient heat is removed from the system to cause condensation and partially saturate the porous medium. Because the control volume surfaces are closed and adiabatic, the initial quantities of air and water mass should remain constant throughout the simulation. Upon partial saturation of a desaturated node, MSTS switches the primary variable for the water mass conservation equation from the the water-vapor mass fraction to the liquid pressure. This problem demonstrates this numerical transition from gas-phase-only conditions to two-phase conditions. The problem requires the coupled solution of the water mass, air mass, and energy conservation equations.

\subsubsection{Problem Domain and Input Parameters}

This problem involves a $10-\mathrm{m}^{3}$ cube of porous media, with a total porosity of 0.50 , rock density of $2650 \mathrm{~kg} / \mathrm{m}^{3}$, and specific heat of $1000 \mathrm{~J} / \mathrm{kg} \mathrm{K}$. The initial gas pressure, liquid saturation, and temperature are $4 \mathrm{MPa}$ (absolute), $2 \times 10^{-8}$, and $280^{\circ} \mathrm{C}$, respectively. A constant internal heat removal rate of $-0.5 \mathrm{MW}$ was applied. All boundary surfaces are adiabatic, no-flow surfaces. A complete description of the problem specifics is given in the "input" file shown in Exhibit 8.3.

\subsubsection{Simulation Results}

Simulation results in terms of the system temperature and liquid saturation are shown in Figure 8.2. The liquid saturation remains at zero (the initial condition) until near 1000 seconds, at which time the numerical switch of primary variables occurs when temperature has declined to a point where phase change from gas to aqueous begins. The simulation is halted at 1875 seconds at a final temperature of $214.7^{\circ} \mathrm{C}$.

\subsubsection{Mass Balance Check}

As for the single-node-evaporation problem, this problem concerns a closed system in which mass must be conserved. The mass balance check is repeated for the condensation problem using Equations (8.2) through (8.4) with values from the single-node-condensation problem (Table 8.3). For the initial conditions, the mass of water in the aqueous phase is computed as 


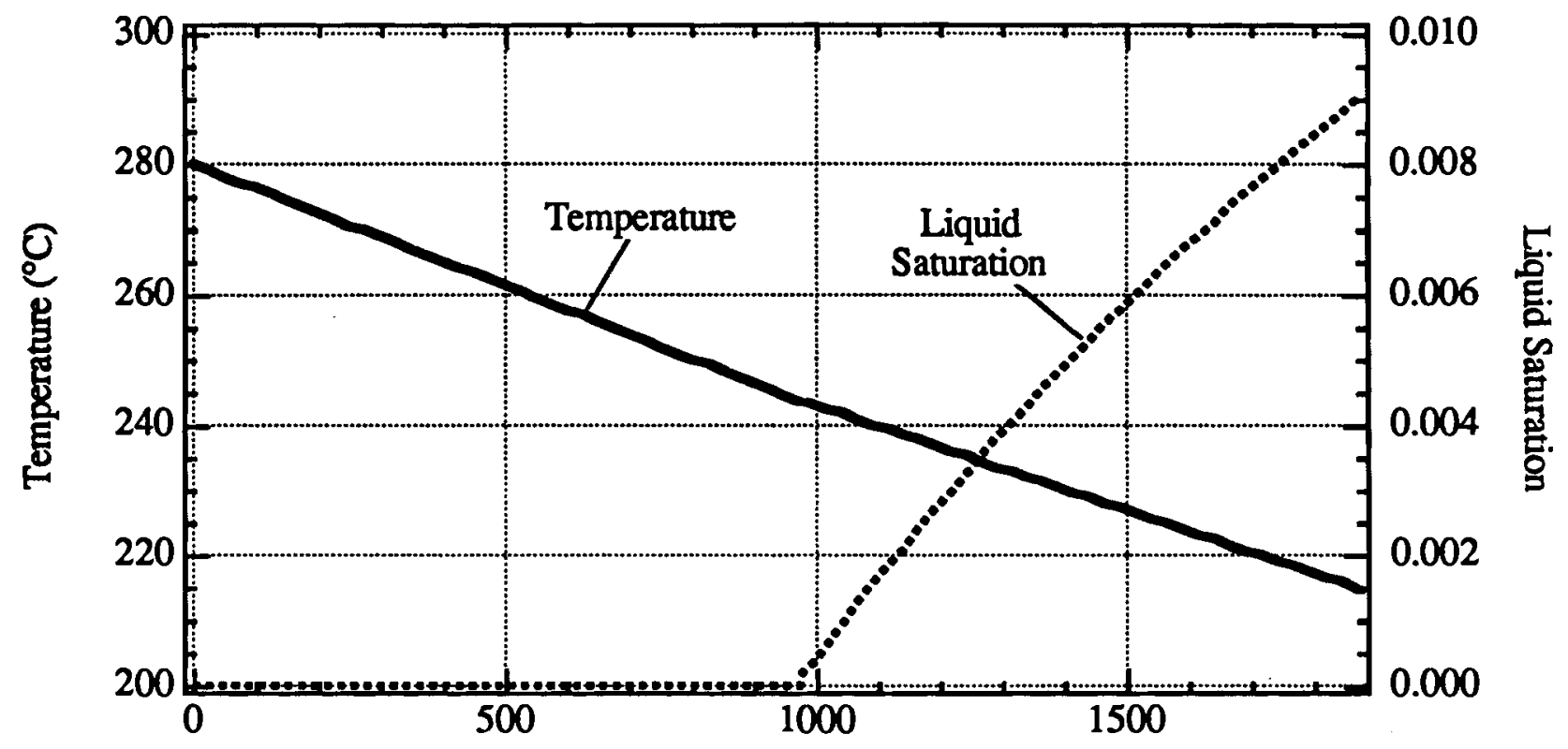

Simulation Time (s)

Figure 8.2. MSTS-Predicted Temperature and Liquid Saturation Variation with Time for the Single-Node-Condensation Problem

Table 8.3. Single-Node-Condensation Problem Initial and Final Conditions Variable Units Initial Conditions Final Conditions

$\begin{array}{llll}V & \mathrm{~m}^{3} & 10.0 & 10.0\end{array}$

$\begin{array}{llll}n_{T} & - & 0.50 & 0.50\end{array}$

$\begin{array}{llll}n_{D} & - & 0.50 & 0.50\end{array}$

$\begin{array}{llll}T & { }^{\circ} \mathrm{C} & 280.00 & 214.68\end{array}$

$\begin{array}{llll}s_{\ell} & - & 0.000000 & 0.009000\end{array}$

$\begin{array}{llll}\rho_{\ell} & \mathrm{kg} / \mathrm{m}^{3} & 746.3 & 847.1\end{array}$

$\begin{array}{llll}\rho_{\mathrm{g}} & \mathrm{kg} / \mathrm{m}^{3} & 18.04 & 10.64\end{array}$

$\begin{array}{llll}x_{\ell}^{w} & - & 1.0000 & 1.0000\end{array}$ 


$$
\begin{aligned}
m_{\ell}^{w} & =\left(n_{T}-n_{D}+s_{\ell} n_{D}\right) V \rho_{\ell} \\
& =[0.50-0.50+(0.0)(0.50)]\left(10 \mathrm{~m}^{3}\right)\left(746.3 \mathrm{~kg} / \mathrm{m}^{3}\right) \\
& =0.0 \mathrm{~kg}
\end{aligned}
$$

(i.e., there is no water in the aqueous phase), while the water mass in the gas phase at time zero is

$$
\begin{aligned}
m_{g}^{w} & =\left(1-s_{\ell}\right) \mathrm{n}_{D} V x_{\ell}^{w} \rho_{g} \\
& =(1-0.0)(0.50)\left(10 \mathrm{~m}^{3}\right)(1.0)\left(18.04 \mathrm{~kg} / \mathrm{m}^{3}\right) \\
& =90.200 \mathrm{~kg}
\end{aligned}
$$

Therefore, the total water mass at the start of the simulation period is $90.200 \mathrm{~kg}$. At the end of the simulation (after 1875 seconds), the temperature is reduced to $214.7^{\circ} \mathrm{C}$ and the water mass in the aqueous phase is

$$
\begin{aligned}
m_{\ell}^{w} & =\left(n_{T}-n_{D}+s_{\ell} n_{D}\right) V \rho_{\ell} \\
& =[0.50-0.50+(0.0090)(0.50)]\left(10 \mathrm{~m}^{3}\right)\left(847.1 \mathrm{~kg} / \mathrm{m}^{3}\right) \\
& =38.120 \mathrm{~kg}
\end{aligned}
$$

while the water mass remaining in the gas phase is

$$
\begin{aligned}
m_{g}^{w} & =\left(1-s_{\ell}\right) n_{D} V x_{\ell}^{w} \rho_{g} \\
& =(1-0.0090)(0.50)\left(10 \mathrm{~m}^{3}\right)(1.0)\left(10.64 \mathrm{~kg} / \mathrm{m}^{3}\right) \\
& =52.721 \mathrm{~kg}
\end{aligned}
$$

Summing, the total water mass in the system at the end of the simulation is $38.120+52.721=$ $90.841 \mathrm{~kg}$. The difference between initial and final mass is $90.200-90.841=-0.641 \mathrm{~kg}$, which is $0.71 \%$ of the initial mass. 
Exhibit 8.3. MSTS Input File for Single-Node-Condensation Problem

\section{Line No. Input File}

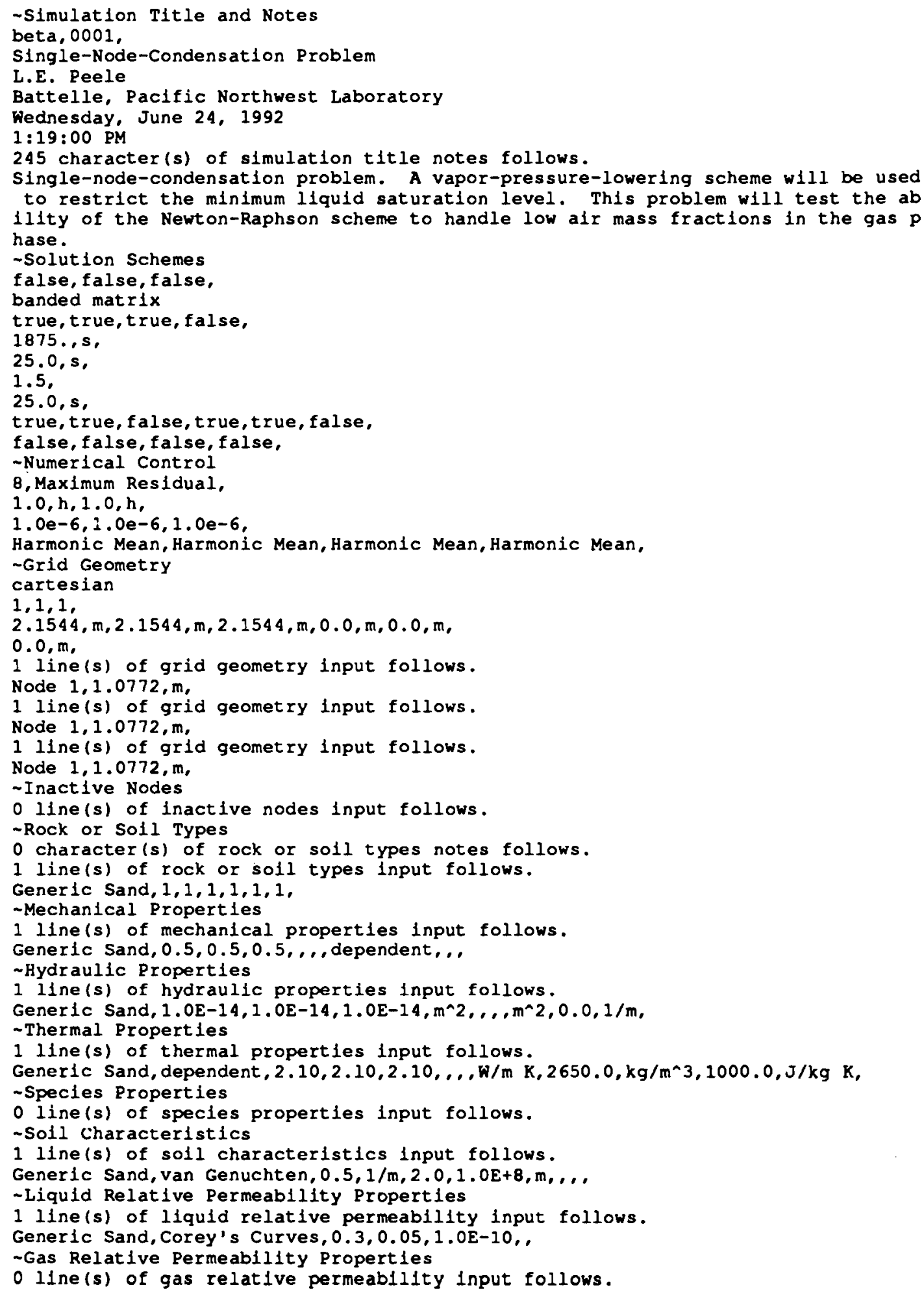




\section{Exhibit 8.3. (Contd)}

\section{Line No. Input File}

64 Liquid Boundary Conditions

650 line(s) of liquid boundary conditions input follows. - Gas Boundary Conditions

o line(s) of gas boundary conditions input follows.

- Vapor Boundary Conditions

o line(s) of vapor boundary conditions input follows.

- Energy Boundary Conditions

o line (s) of energy boundary conditions input follows.

- Species Boundary Conditions

o line(s) of species boundary conditions input follows.

- Initial Conditions

Liquid Saturation Gas Pressure,

4 line(s) of initial conditions input follows.

Gas Pressure, 4.0E+06, Pa (absolute), 1/m, 1/m, 1/m, 1, 1, 1, 1, 1, 1 ,

Liquid Saturation, $0.0, \ldots, 1 / \mathrm{m}, 1 / \mathrm{m}, 1 / \mathrm{m}, 1,1,1,1,1,1$,

Temperature, 280.0, Celsius, ,1/m, $1 / \mathrm{m}, 1 / \mathrm{m}, 1,1,1,1,1,1$,

Vapor Mass Fraction, $1.0, \ldots, 1 / \mathrm{m}, 1 / \mathrm{m}, 1 / \mathrm{m}, 1,1,1,1,1,1$,

- Sources sinks

1 line(s) of sources sinks input follows.

Thermal Energy, Constant, $-5.0 \mathrm{E}+4, \mathrm{~W} / \mathrm{m} \wedge 3, \ldots, 0, \mathrm{~s}, 1875.0, \mathrm{~s}, 1,1,1,1,1,1$,

- Liquid Pathline Trace

0 line(s) of liquid pathline trace input follows.

Output Control

$1,1,1,1,1,1$, zxplane,

0 line(s) of output control input follows.

$1,1,1, \ldots, \ldots, \ldots, 1$,

3 line (s) of output control input follows.

Liquid Pressure,

Temperature,

Liquid Saturation,

8 Ine(s) of output control input follows.

Liquid Pressure,

Gas Pressure,

Temperature,

Liquid Saturation,

Gas Phase Vapor Mass Fraction,

Liquid Phase Rel. Permeability.

Liquid Density.

Gas Density,

3 line(s) of output control input follows.

Liquid Pressure,

Temperature,

Liquid Saturation,

-Surface Flux Integrator

0 line(s) of surface flux integrator input follows.

rock List

1 line(s) of rock list input follows.

Generic Sand,

$\sim$ 


\subsection{Flow from Hot Two-Phase Conditions}

This problem is concerned with two-phase flow between two adjacent cubes of porous medium with sharply differing initial conditions. The problem starts with both nodes in two-phase conditions; however, one node is nearly saturated with relatively cool water and the other is nearly desaturated at an elevated temperature. The problem involves simulating imbibition and vaporization of the liquid water from relatively cool saturated conditions to hot dry conditions. The control volume surfaces surrounding the two nodes are adiabatic and impermeable to fluid flow. The problem proceeds until equilibrium conditions are reached. The problem demonstrates the conservation of water mass, air mass, and thermal energy for two-phase flow conditions. An energy balance error analysis is presented from the simulation results of this problem.

\subsubsection{Problem Domain and Input Parameters}

The problem involves two cubes of porous medium, both with porosity 0.50 , volume $10 \mathrm{~m}^{3}$, and hydraulic conductivity $1.0 \times 10^{-14} \mathrm{~m}^{2}$. The rock thermal conductivity, density, and specific heat are $2.10 \mathrm{~W} / \mathrm{m} \mathrm{K}, 2650 \mathrm{~kg} / \mathrm{m}^{3}$, and $1000 \mathrm{~J} / \mathrm{kg} \mathrm{K}$, respectively. The initial conditions for the first (west) cube are: gas pressure, $1.0 \times 10^{5} \mathrm{~Pa}$ (absolute); liquid saturation, 0.999 ; temperature, $99.5^{\circ} \mathrm{C}$. For the second (east) cube, the initial conditions are: gas pressure, $9.9 \times 10^{6} \mathrm{~Pa}$ (absolute); liquid saturation, 0.001 ; temperature, $310.0^{\circ} \mathrm{C}$. The input file is shown in Exhibit 8.4.

\subsubsection{Energy Balance Check}

Let $q_{i}$ represent the thermal energy in computational node $i ; q_{i}$ is the sum of the rock or soil internal energy, aqueous-phase-water internal energy, water-vapor internal energy, and air internal energy (the contribution from air dissolved in the aqueous phase is negligible):

$$
\begin{aligned}
q_{i}= & \left(1-n_{T}\right) c_{s} \rho_{s} T+s_{\ell} \rho_{\ell} \eta_{D} u_{\ell}^{w}+ \\
& s_{g} \rho_{g} x_{g}^{w} n_{D} u_{g}^{w}+s_{g} \rho_{g}\left(1-x_{g}^{w}\right) n_{D} u_{g}^{a}
\end{aligned}
$$

The total energy in the problem domain at time $t$ is the sum of all computational nodes, or $\Sigma q_{i}$.

Table 8.4 lists the values of all of the parameters required by Equation (8.5) for both initial and final conditions. All parameter values except the internal energies $\left(u_{\ell}^{w}, u_{g}^{w}, u_{g}^{a}\right)$ were obtained from MSTS-generated "plot" files using the "input" file shown in Exhibit 8.4. The plot file for initial conditions was obtained by changing the Initial Conditions Only option in the "input" file (Exhibit 8.4, Solution Schemes card, line 11, first option) from "false" to "true" and running MSTS again. The values for the internal energies of water in the aqueous and gas phases were 
Table 8.4. Hot Two-Phase Flow Problem Initial and Final Conditions

\begin{tabular}{|c|c|c|c|c|c|}
\hline \multirow[b]{2}{*}{ Variable } & \multirow[b]{2}{*}{ Units } & \multicolumn{2}{|c|}{ Initial Conditions } & \multicolumn{2}{|c|}{ Final Conditions } \\
\hline & & West Node & East Node & West Node & East Node \\
\hline$c_{s}$ & $\mathrm{~J} / \mathrm{kg} \mathrm{K}$ & 1000.0 & 1000.0 & 1000.0 & 1000.0 \\
\hline$n_{T}$ & - & 0.50 & 0.50 & 0.50 & 0.50 \\
\hline$\rho_{s}$ & $\mathrm{~kg} / \mathrm{m}^{3}$ & 2650.0 & 2650.0 & 2650.0 & 2650.0 \\
\hline$\rho_{l}$ & $\mathrm{~kg} / \mathrm{m}^{3}$ & 958.48 & 690.68 & 898.49 & 898.49 \\
\hline$\rho_{g}$ & $\mathrm{~kg} / \mathrm{m}^{3}$ & 0.59213 & 54.724 & 4.2060 & 4.2060 \\
\hline$s_{\ell}$ & - & 0.999 & 0.001 & 0.56160 & 0.56160 \\
\hline$s_{8}$ & - & 0.001 & 0.999 & 0.43840 & 0.43840 \\
\hline$T$ & ${ }^{\circ} \mathrm{C}$ & 99.5 & 310.0 & 168.83 & 168.83 \\
\hline$u_{\ell}^{\mathrm{w}}$ & $\mathrm{kJ} / \mathrm{kg}$ & 416.83 & 1387.1 & 713.24 & 713.24 \\
\hline$u_{g}^{w}$ & $\mathrm{~kJ} / \mathrm{kg}$ & 2505.91 & 2546.4 & 2575.56 & 2575.56 \\
\hline$u_{g}^{a}$ & $\mathrm{~kJ} / \mathrm{kg}$ & 266.39 & 421.96 & 316.79 & 316.79 \\
\hline$x_{g}^{w}$ & - & 0.99290 & 0.99686 & 0.95439 & 0.95439 \\
\hline
\end{tabular}

obtained from steam tables (Table A.1, van Wylen and Sonntag 1985). The values for the internal energy of air in the gas phase were obtained from a table of ideal gas integrals for air (Table A.10, van Wylen and Sonntag 1985).

Computing the total internal energy per unit volume for the west node at initial conditions:

$$
\begin{aligned}
q_{\text {west }}^{\text {init }}= & (1-0.50)\left(1000 \frac{\mathrm{J}}{\mathrm{kg} \mathrm{K}}\right)\left(2650 \frac{\mathrm{kg}}{\mathrm{m}^{3}}\right)(372.65 \mathrm{~K}) \\
& +(0.999)\left(958.48 \frac{\mathrm{kg}}{\mathrm{m}^{3}}\right)(0.50)\left(416,830 \frac{\mathrm{J}}{\mathrm{kg}}\right) \\
& +(0.001)\left(0.59213 \frac{\mathrm{kg}}{\mathrm{m}^{3}}\right)(0.99290)(0.50)\left(2,505,910 \frac{\mathrm{J}}{\mathrm{kg}}\right) \\
& +(0.001)\left(0.59213 \frac{\mathrm{kg}}{\mathrm{m}^{3}}\right)(1-0.99290)(0.50)\left(266,390 \frac{\mathrm{J}}{\mathrm{kg}}\right) \\
= & 6.9332 \times 10^{8} \mathrm{~J} / \mathrm{m}^{3}
\end{aligned}
$$


which, when multiplied by the node volume $\left(10 \mathrm{~m}^{3}\right)$, yields the total node energy at time zero of $6.9332 \times 10^{9} \mathrm{~J}$. Repeating for the east node, we obtain

$$
\begin{aligned}
q_{\text {east }}^{\text {init }}= & (1-0.50)\left(1000 \frac{\mathrm{J}}{\mathrm{kg} \mathrm{K}}\right)\left(2650 \frac{\mathrm{kg}}{\mathrm{m}^{3}}\right)(583.15 \mathrm{~K}) \\
& +(0.001)\left(690.68 \frac{\mathrm{kg}}{\mathrm{m}^{3}}\right)(0.50)\left(1,387,100 \frac{\mathrm{J}}{\mathrm{kg}}\right) \\
& +(0.999)\left(54.724 \frac{\mathrm{kg}}{\mathrm{m}^{3}}\right)(0.99686)(0.50)\left(2,546,400 \frac{\mathrm{J}}{\mathrm{kg}}\right) \\
& +(0.999)\left(54.724 \frac{\mathrm{kg}}{\mathrm{m}^{3}}\right)(1-0.99686)(0.50)\left(421,960 \frac{\mathrm{J}}{\mathrm{kg}}\right) \\
= & 8.4258 \times 10^{8} \mathrm{~J} / \mathrm{m}^{3}
\end{aligned}
$$

for which the total energy is therefore $8.4258 \times 109 \mathrm{~J}$. The total energy in the problem domain is the sum of these two nodes, or 15,359.0 MJ. For the final conditions, the two nodes are in equilibrium; hence only one calculation is necessary:

$$
\begin{aligned}
q^{\text {final }}= & (1-0.50)\left(1000 \frac{\mathrm{J}}{\mathrm{kg} \mathrm{K}}\right)\left(2650 \frac{\mathrm{kg}}{\mathrm{m}^{3}}\right)(441.98 \mathrm{~K}) \\
& +(0.56160)\left(898.49 \frac{\mathrm{kg}}{\mathrm{m}^{3}}\right)(0.50)\left(713,240 \frac{\mathrm{J}}{\mathrm{kg}}\right) \\
& +(0.43840)\left(4.2060 \frac{\mathrm{kg}}{\mathrm{m}^{3}}\right)(0.95439)(0.50)\left(2,575,560 \frac{\mathrm{J}}{\mathrm{kg}}\right) \\
& +(0.43840)\left(4.2060 \frac{\mathrm{kg}}{\mathrm{m}^{3}}\right)(1-0.95439)(0.50)\left(316,790 \frac{\mathrm{J}}{\mathrm{kg}}\right) \\
= & 7.6785 \times 10^{8} \mathrm{~J} / \mathrm{m}^{3}
\end{aligned}
$$

This applies for both nodes, or a total volume of $20 \mathrm{~m}^{3}$; hence the total energy in the system predicted by MSTS at the end of the simulation for equilibrium conditions is $15,357.0 \mathrm{MJ}$. The difference between the total energy in the domain at the beginning and at the end of the simulation $(2.0 \mathrm{MJ})$ is the error introduced during the simulation by MSTS through numerical approximation, and is equal to $0.013 \%$ of the initial total energy. 
Exhibit 8.4. MSTS Input File for Hot Two-Phase Problem

\section{Line No. Input File}

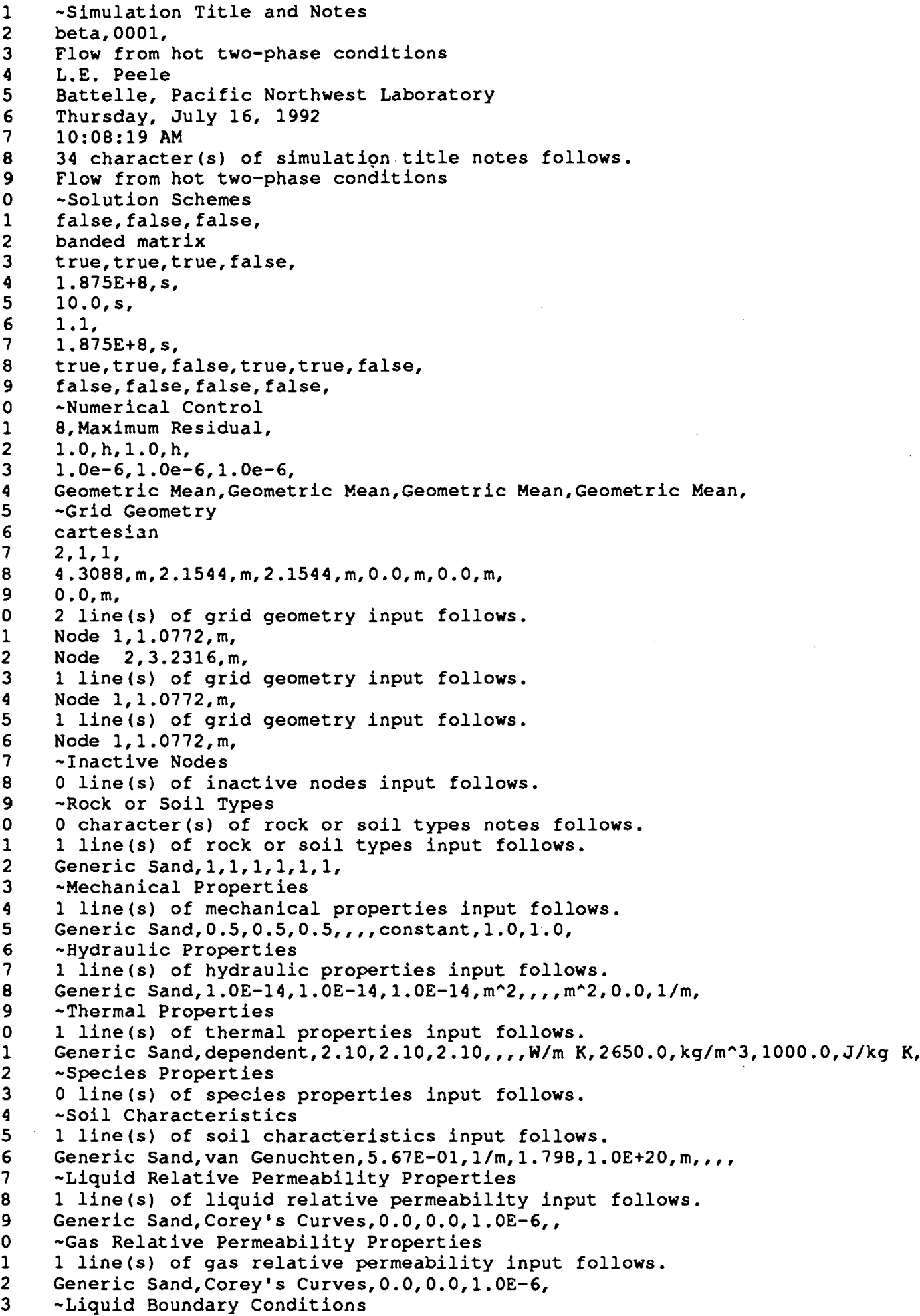




\section{Exhibit 8.4. (Contd)}

\section{Line No. Input File}

40 line(s) of liquid boundary conditions input follows. - Gas Boundary Conditions

0 line(s) of gas boundary conditions input follows.

- Vapor Boundary Conditions

o line(s) of vapor boundary conditions input follows.

- Energy Boundary Conditions

0 line(s) of energy boundary conditions input follows.

- Species Boundary Conditions

o line(s) of species boundary conditions input follows.

- Initial Conditions

Liquid Saturation Gas Pressure,

6 line(s) of initial conditions input follows.

Gas Pressure, 1.0E+05, Pa (absolute), , 1/m, 1/m, 1/m, 1, 1, 1, 1, 1, 1,

Gas Pressure, 99.0E+05, Pa (absolute),.,1/m, 1/m, 1/m, 2, 2, 1, 1, 1, 1,

Liquid Saturation, $0.001, \ldots, 1 / \mathrm{m}, 1 / \mathrm{m}, 1 / \mathrm{m}, 2,2,1,1,1,1$,

Liquid Saturation, $0.999, \ldots, 1 / \mathrm{m}, 1 / \mathrm{m}, 1 / \mathrm{m}, 1,1,1,1,1,1$,

Temperature, 310.0 , Celsius, $1 / \mathrm{m}, 1 / \mathrm{m}, 1 / \mathrm{m}, 2,2,1,1,1,1$,

Temper ature, 99.5, Celsius, ,1/m, , 1/m, , 1/m, 1, 1, 1, 1, 1, 1,

- Sources \& Sinks

0 line(s) of sources sinks input follows.

- Liquid Pathline Trace

0 line(s) of liquid pathline trace input follows.

- Output Control

$1,2,1,1,1,1,2 x p l a n e$

o line(s) of output control input follows.

$1,1,1, \ldots, \ldots, \ldots, 1$,

9 line (s) of output control input follows.

Liquid Pressure.

Gas Pressure,

Temperature,

Liquid Saturation,

Gas Saturation,

Liquid Phase Air Mass Fraction,

Gas Phase Vapor Mass Fraction,

Liquid Density.

Gas Density,

9 line(s) of output control input follows.

Liquid Pressure,

Gas Pressure.

Temperature,

Liquid Saturation,

Gas Saturation.

Liquid Phase Air Mass Fraction,

Gas Phase Vapor Mass Fraction,

Liquid Density.

Gas Density,

3 line(s) of output control input follows.

Liquid Pressure,

Temperature.

Liquid Saturation,

-Surface Flux Integrator

0 line(s) of surface flux integrator input follows.

r Rock L1st

1 line(s) of rock list input follows.

Generic Sand, 


\subsection{Philip's Vertical Infiltration Problem}

This problem demonstrates that the MSTS code correctly computes a wetting front propagation with gravitational effects. Results obtained with MSTS are compared to those of a semi-analytic solution reported by Philip (1957).

\subsubsection{Purpose}

The purpose of this one-dimensional, time-dependent, unsaturated, single-phase, vertical infiltration problem is to demonstrate that MSTS correctly solves the water conservation equation for a wetting front in a semi-infinite porous medium. The problem involves a $15-\mathrm{cm}$ column filled with Yolo light clay, first described by Moore (1939). The governing equation for this problem is the one-dimensional form of Richards' Equation:

$$
C(\psi)=\frac{\partial \psi}{\partial t}=\frac{\partial}{\partial z}\left[k(\psi)\left(\frac{\partial \psi}{\partial z}-1\right)\right]
$$

where $\psi=$ tension head (L)

$$
\begin{aligned}
k(\psi) & =\text { unsaturated hydraulic conductivity }\left(\mathrm{L} \mathrm{t}^{-1}\right) \\
t & =\text { time (t) } \\
z & =\text { distance along the vertical coordinate }(\mathrm{L}) .
\end{aligned}
$$

The specific moisture capacity $C(\psi)$ is given by

$$
C(\psi)=\frac{\partial \theta}{\partial \psi}
$$

where $\theta$ is the volumetric moisture content.

The analytic results used for this comparison were generated by the INFIL computer code (ElKadi 1987) for conditions at the end of 2.0 hours of infiltration.

\subsubsection{Model Domain and Input Parameters}

The functional relationships used for the moisture-retention curves are described by Haverkamp et al. (1977). The formulae for these relationships are provided in Section 7.3. The parameter values used are as follows: 


$$
\begin{aligned}
& \theta_{s}=0.495 \text { saturated volumetric moisture content } \\
& \theta_{r}=0.124 \text { residual volumetric moisture content } \\
& k_{s}=0.04428 \text { saturated hydraulic conductivity }(\mathrm{cm} / \mathrm{h}) \\
& \alpha=739 \quad \text { curve-fitting parameter } \\
& \beta=4.0 \quad \text { curve-fitting parameter } \\
& A=124.6 \quad \text { curve-fitting parameter } \\
& B=1.77 \quad \text { curve-fitting parameter }
\end{aligned}
$$

These parameter values were entered into the Soil Characteristics card (Exhibit 8.5, lines 153-155) and Liquid Relative Permeability card (Exhibit 8.5, lines 156-158).

The initial conditions for the problem are a uniform pressure head of $-601.8 \mathrm{~cm}$, which corresponds to a moisture content of 0.238 . The boundary conditions are saturation at the upper boundary and constant pressure at the initial value for the lower boundary. Thus, infiltration begins at time zero from the saturated upper boundary.

A total of 99 nodes are specified in the $z$-direction to represent the $15 \mathrm{~cm}$ of soil (Exhibit 8.5, lines 27-135). The distribution of these nodes was made to match those available from the INFIL computer code output, and is not precisely uniform as it would be if it were assigned by the MSTS Graphical Input's Uniform option.

Because MSTS is a multiphase code, we wish to express the the initial pressure as a pressure rather than a pressure head. To convert this, assuming standard conditions $\left(20^{\circ} \mathrm{C}\right)$, we compute

$$
\begin{aligned}
P_{\ell} & =P_{8}+P_{c a p} \\
& =101,325 \mathrm{~Pa}+\psi \rho_{\ell} g \\
& =101,325 \mathrm{~Pa}-\left[(601.8 \mathrm{~cm})\left(\frac{1 \mathrm{~m}}{100 \mathrm{~cm}}\right)\right]\left(998.32 \frac{\mathrm{kg}}{\mathrm{m}^{3}}\right)\left(9.81 \frac{\mathrm{m}}{\mathrm{s}^{2}}\right) \\
& =101,325 \mathrm{~Pa}-58,937.4 \mathrm{~Pa} \\
& =42,387.6 \mathrm{~Pa}
\end{aligned}
$$

where $P_{\ell}$ is the liquid-phase pressure, $P_{g}$ is the gas-phase (atmospheric) pressure, $P_{c a p}$ is the capillary pressure, and $\psi$ is the tension head (negative of pressure head). This result is entered as liquid pressure for the initial conditions (Exhibit 8.5, line 177), along with the uniform gas pressure of 101,325 Pa (absolute), which is the atmospheric pressure (Exhibit 8.5, line 176). 


\subsubsection{Simulation Results and Comparison to Semi-Analytic Solution}

The Philip's vertical infiltration problem was simulated by MSTS in 183 time steps. The input file was also modified to use the geometric mean and arithmetic mean techniques for computing internodal hydraulic conductivities. The liquid pressures predicted by MSTS for all three averaging techniques are compared to the Philip's analytical solution in Figure 8.3 in terms of liquid pressure head. Table 8.5 summarizes the relative root mean squares computed for the liquid saturation predictions of the three MSTS solutions. The RRMS for the harmonic mean technique achieved an "excellent" rating according to the criteria set forth in Baca and Magnuson (1990) while the RRMS for the geometric and arithmetic averaging techniques were rated "good."

Table 8.5. Comparison of MSTS Liquid Saturation Predictions to Philip's Semi-Analytical Solution for Vertical Infiltration of Water Into Yolo Light Clay After 2 Hours

$\begin{array}{cc}\text { Hydraulic Conductivity Averaging Technique } & \text { RRMS } \\ \text { Harmonic } & 0.0093 \\ \text { Geometric } & 0.0124 \\ \text { Arithmetic } & 0.0154\end{array}$




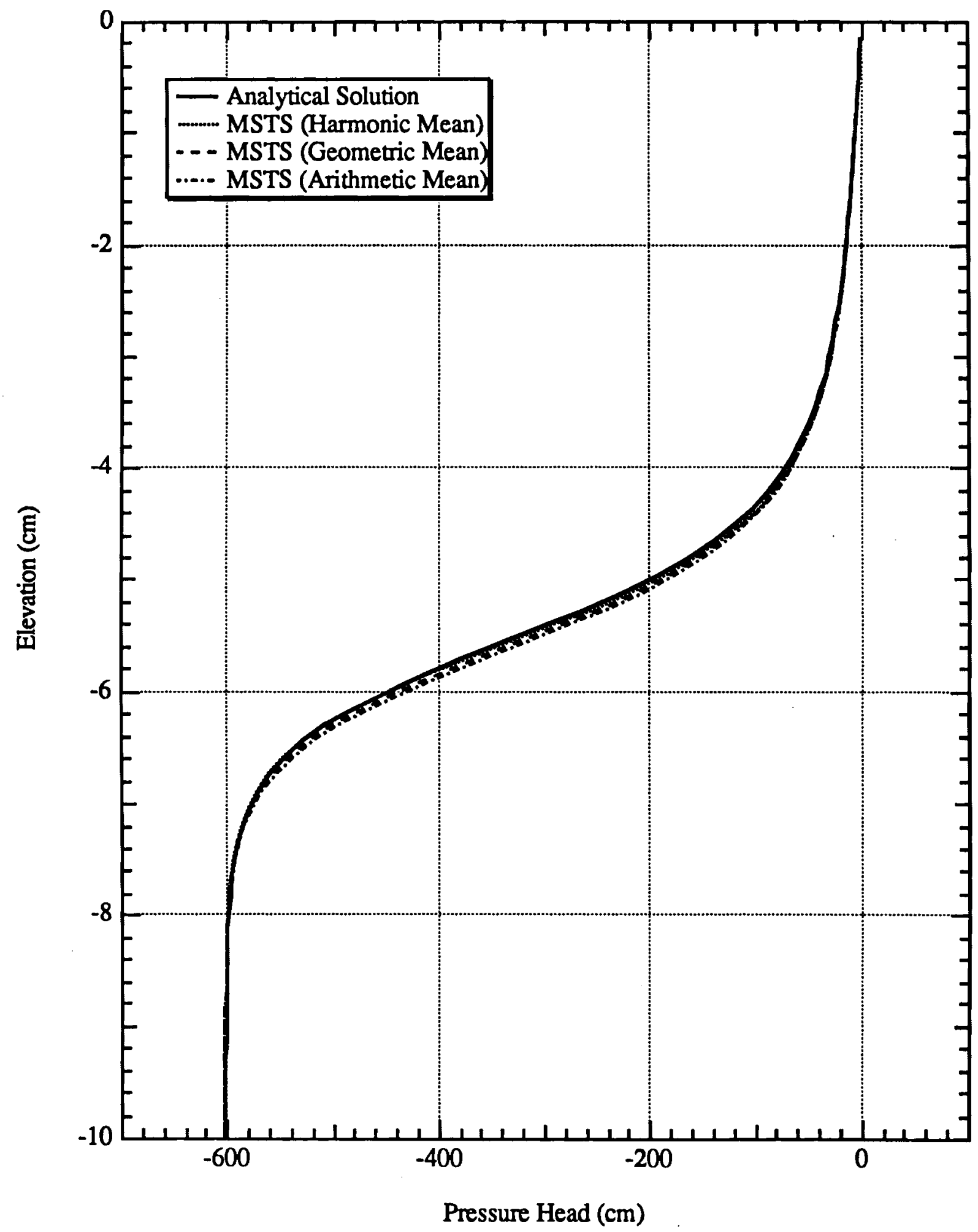

Figure 8.3. Comparison of MSTS Numerical Solutions to the Analytical Solution of the Philip's Vertical Infiltration Problem After 2 Hours of Infiltration 


\section{Exhibit 8.5. MSTS Input File for Philip's Vertical Infiltration Problem}

\section{Line No. Input File}

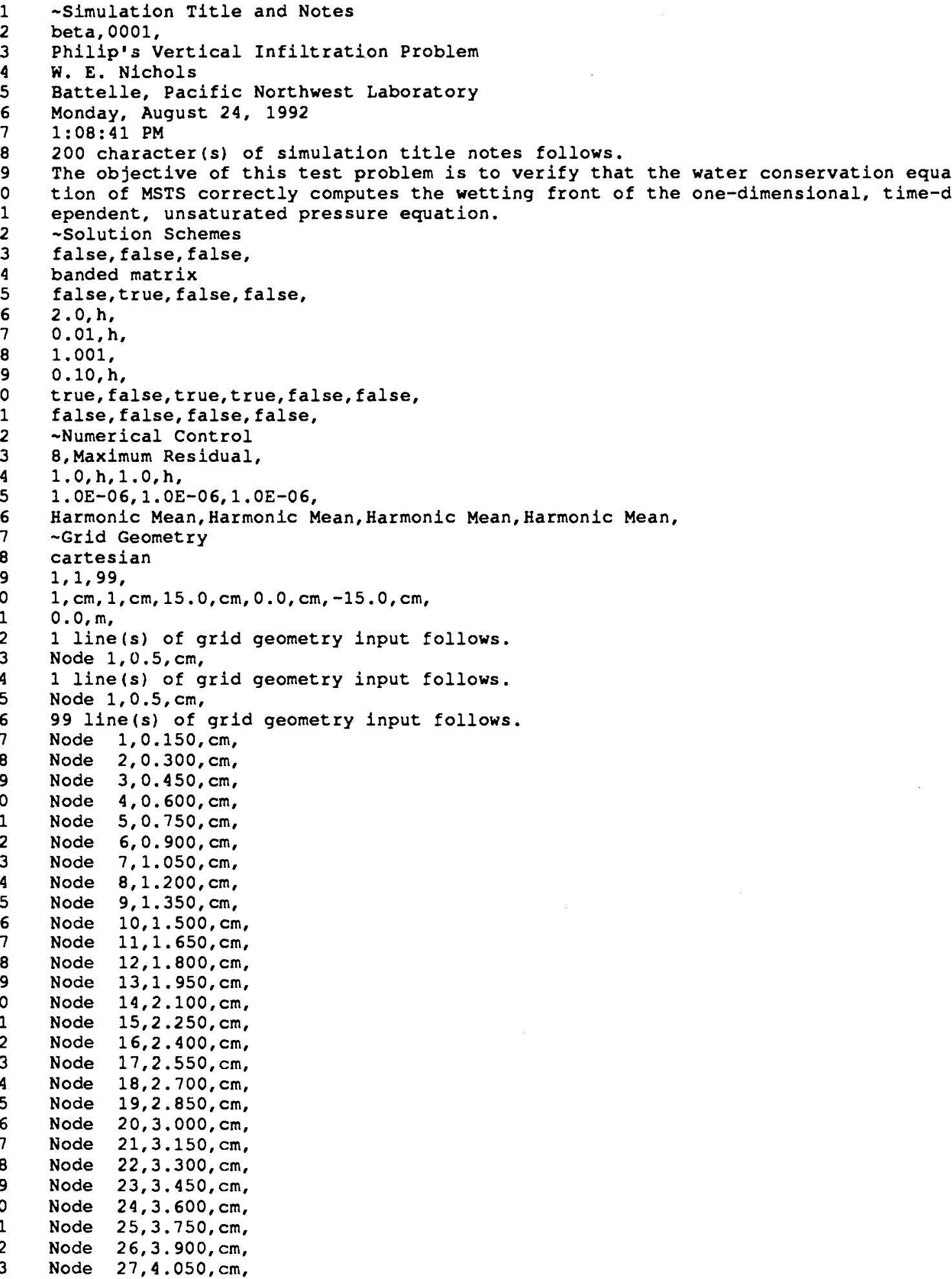


Exhibit 8.5. (Contd)

\section{Line No. Input File}

\begin{tabular}{|c|c|}
\hline 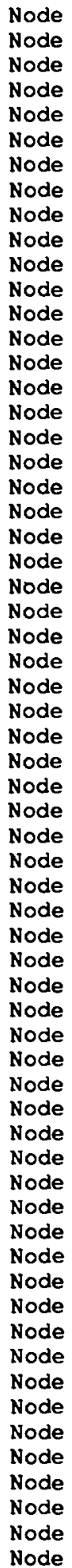 & 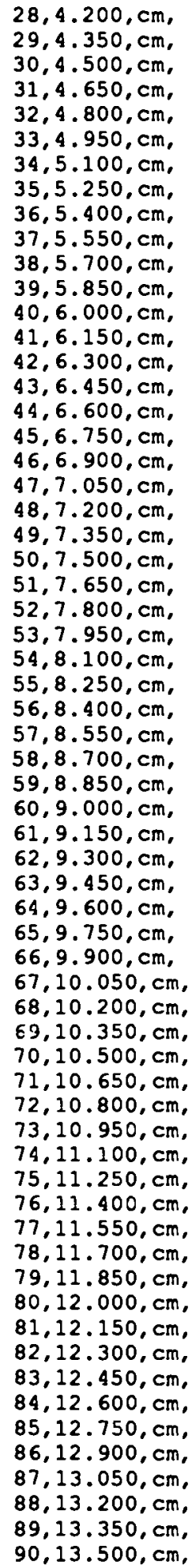 \\
\hline
\end{tabular}




\section{Exhibit 8.5. (Contd)}

\section{Line No. Input File}

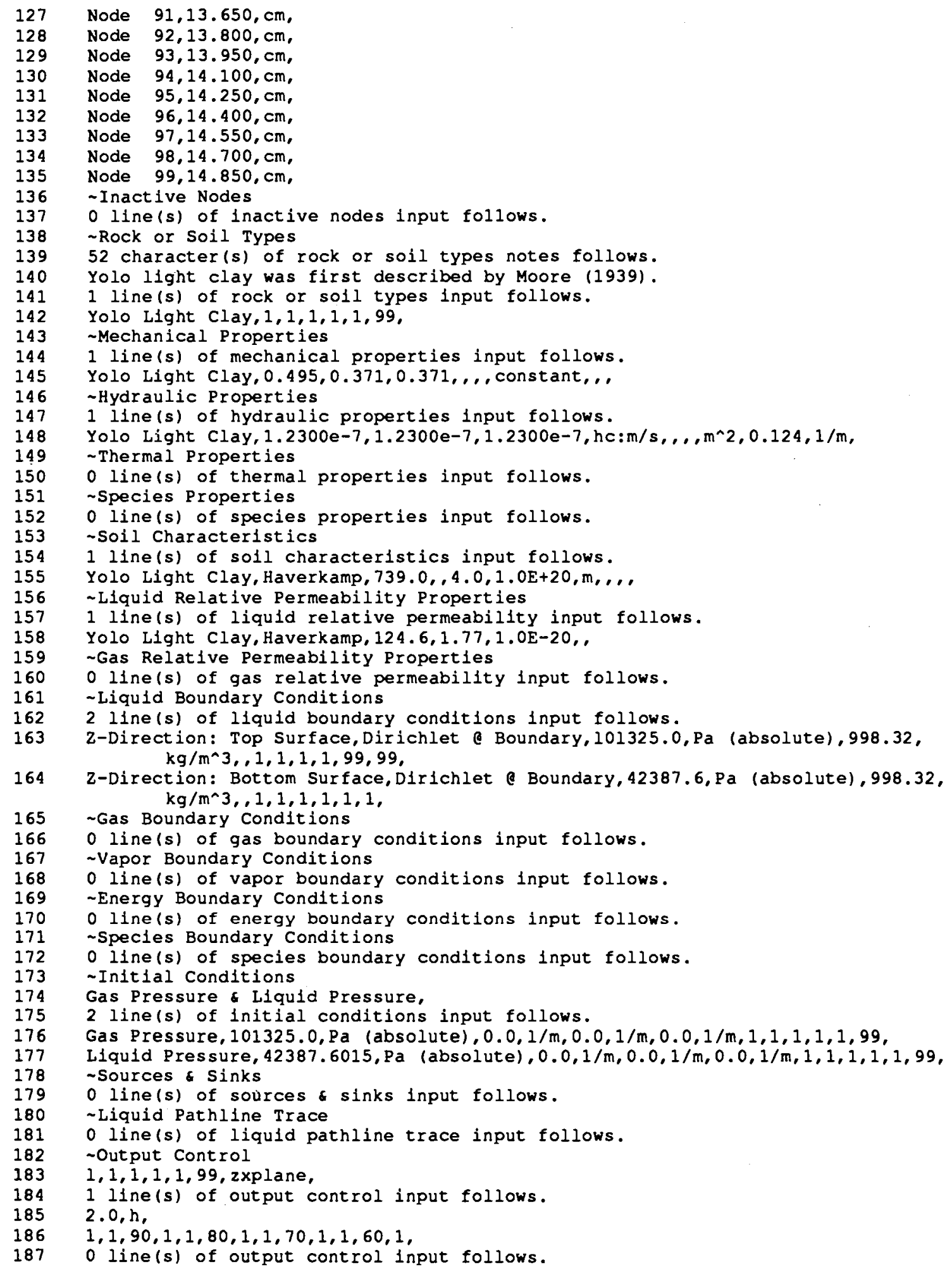




\section{Exhibit 8.5. (Contd)}

\section{Line No Input File}

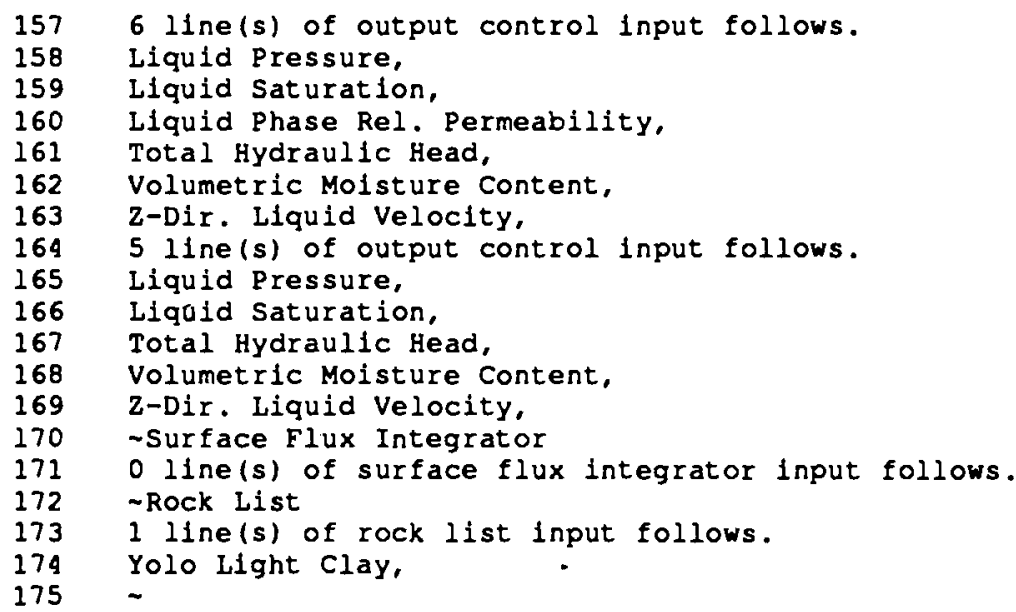




\subsection{Two-Dimensional Infiltration}

This is a two-dimensional infiltration problem proposed by Ross et al. (1982) that is concerned with liquid flow through a two-dimensional rectangular section $(10 \mathrm{~cm}$ high by $15 \mathrm{~cm}$ wide) of partially saturated soil with uniform initial conditions. A wetting surface is propagated through the domain by imposing a wetting surface on a portion of a boundary and allowing the water introduced at the boundary to infiltrate over time. The purpose of the problem is to demonstrate how MSTS propagates a wetting front in two dimensions with gravitational effects.

\subsubsection{Problem Domain and Input Parameters}

The top $4 \mathrm{~cm}$ of the left boundary is maintained at a prescribed capillary (tension) head of $-\psi=$ $6-z(6 \mathrm{~cm} \leq z \leq 10 \mathrm{~cm})$. The whole right boundary is maintained at a prescribed capillary (tension) head of $-\psi=90 \mathrm{~cm}$. All other boundary surfaces are defined as zero-flux surfaces. Figure 8.4 provides a schematic of the problem and illustrates the discretization and boundary condition placement (unmarked boundaries are zero-flux).

The input file for this problem is shown in Exhibit 8.6. The soil-moisture tension characteristic and relative-permeability curves for the soil are provided as tabular input; these are shown graphically in Figure 8.5.

\subsubsection{Simulation Results}

Figure 8.6 shows two capillary pressure profiles, along the top $(z=9.5 \mathrm{~cm})$ and the bottom $(z$ $=0.5 \mathrm{~cm}$ ) of the domain at the same time. Figure 8.7 shows the liquid saturation contours for the domain at the end of the simulation at time 0.508 day. Ross et al. (1982) only proposed this problem; they did not provide a solution (it had not yet been simulated). Pruess (1987) demonstrated the solution to this problem using the TOUGH code. The results reported here are essentially the same as those reported by Pruess (1987). 


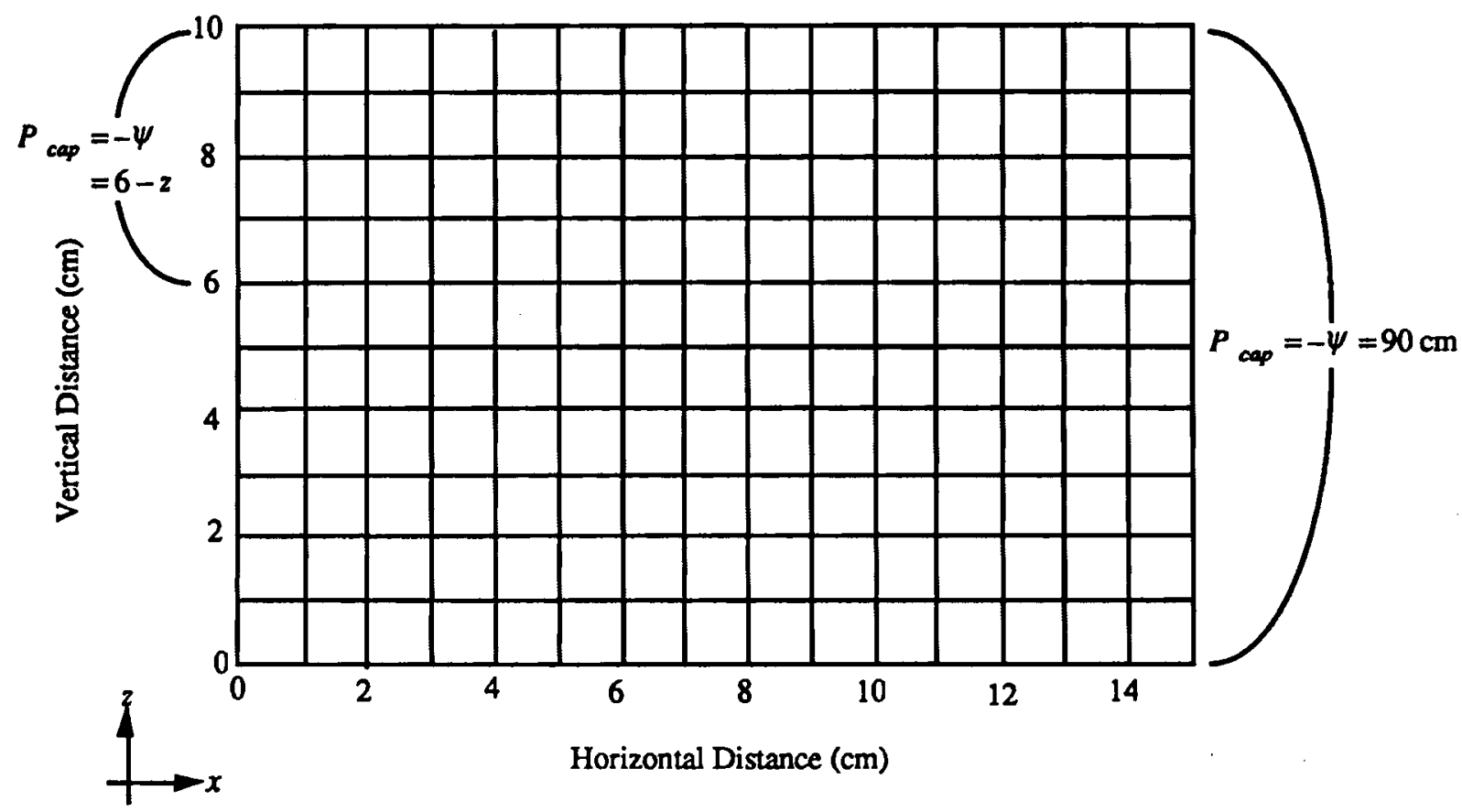

Figure 8.4. Two-Dimensional Infiltration Problem Geometry, Discretization, and Boundary Conditions

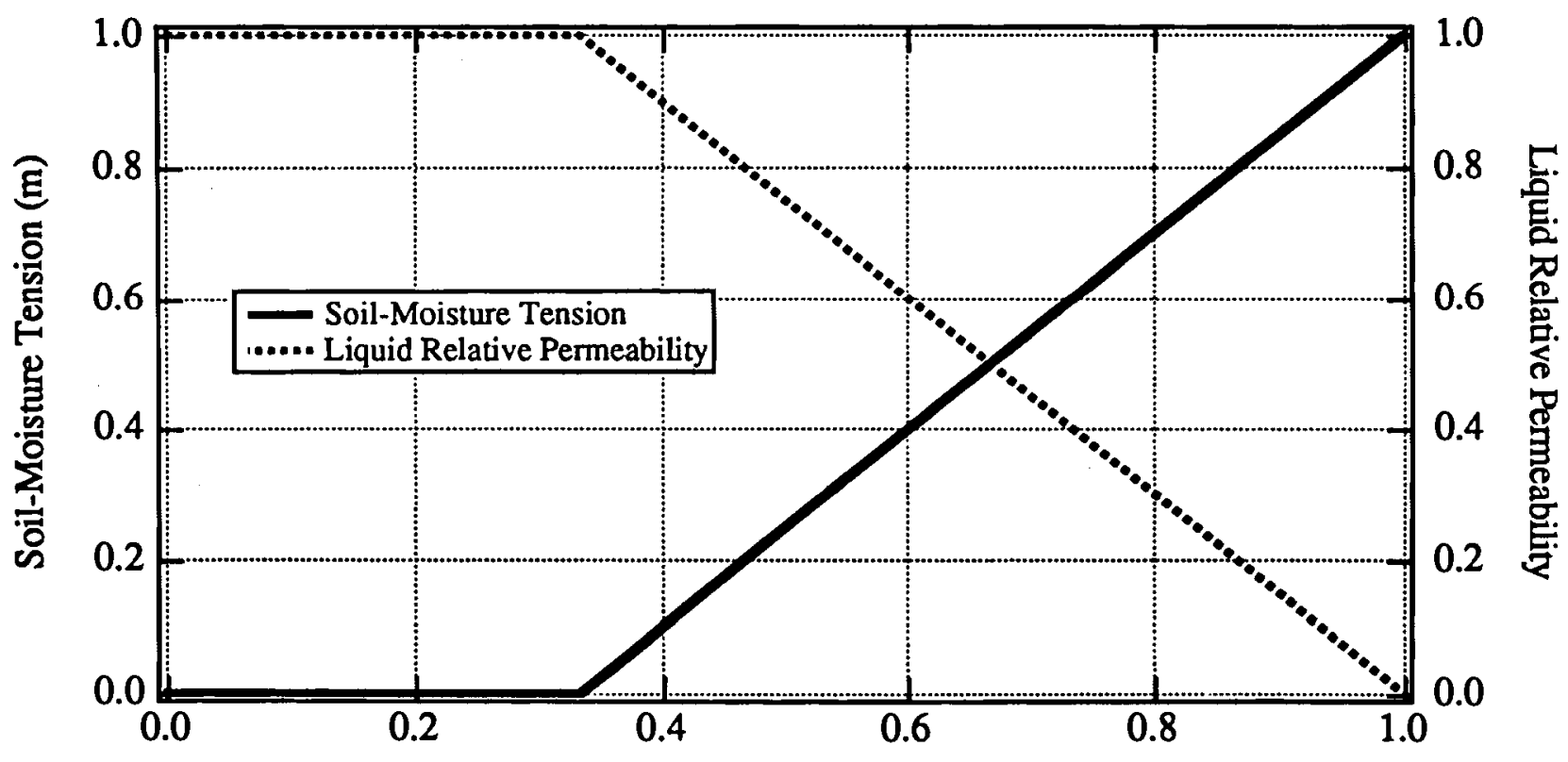

Liquid Saturation

Figure 8.5. Functional Relationships Among Liquid Saturation, Liquid Pressure, and Liquid Relative Permeability for the Two-Dimensional Infiltration Problem 


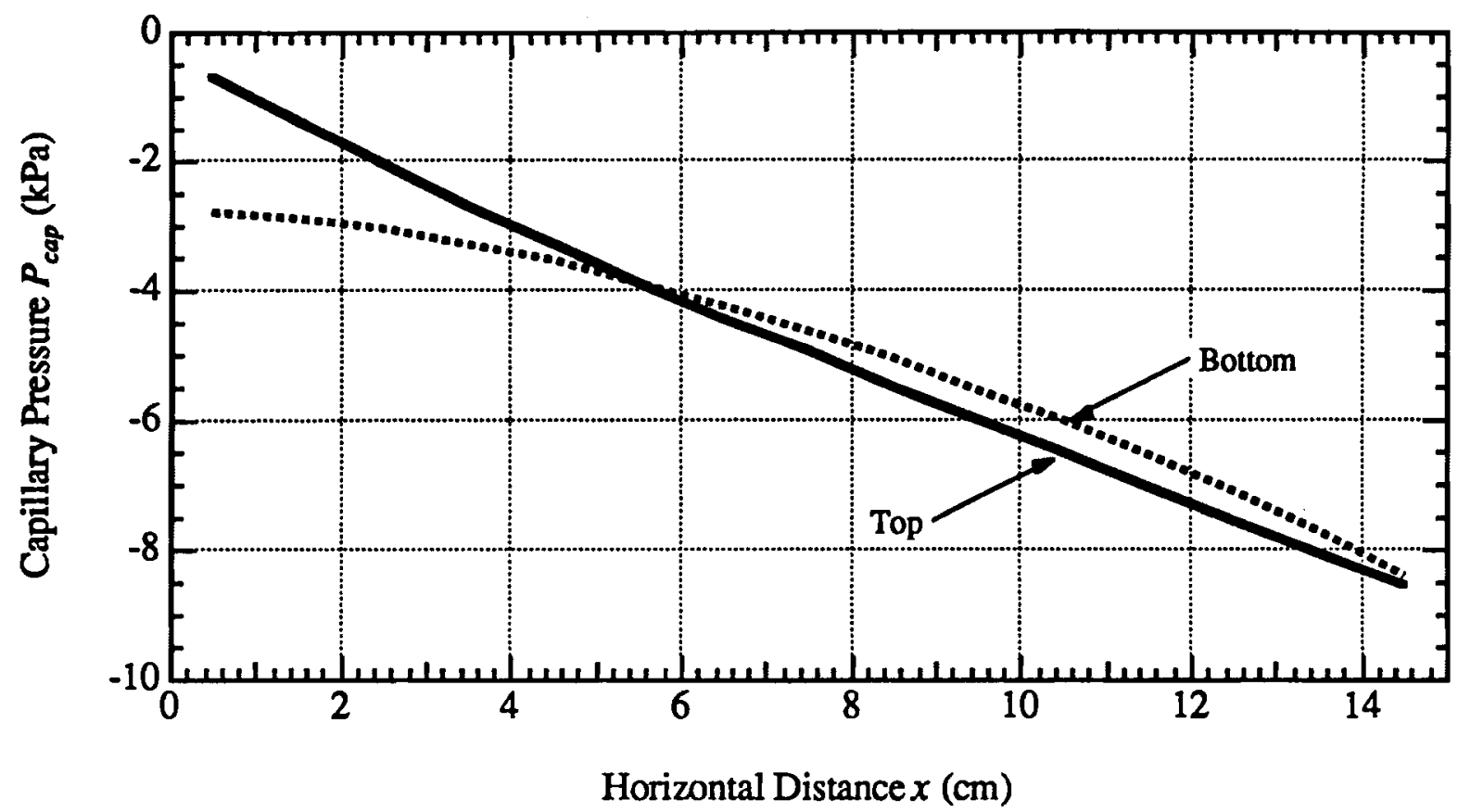

Figure 8.6. Capillary Pressure Profiles at 0.508 Day for Two-Dimensional Infiltration Problem

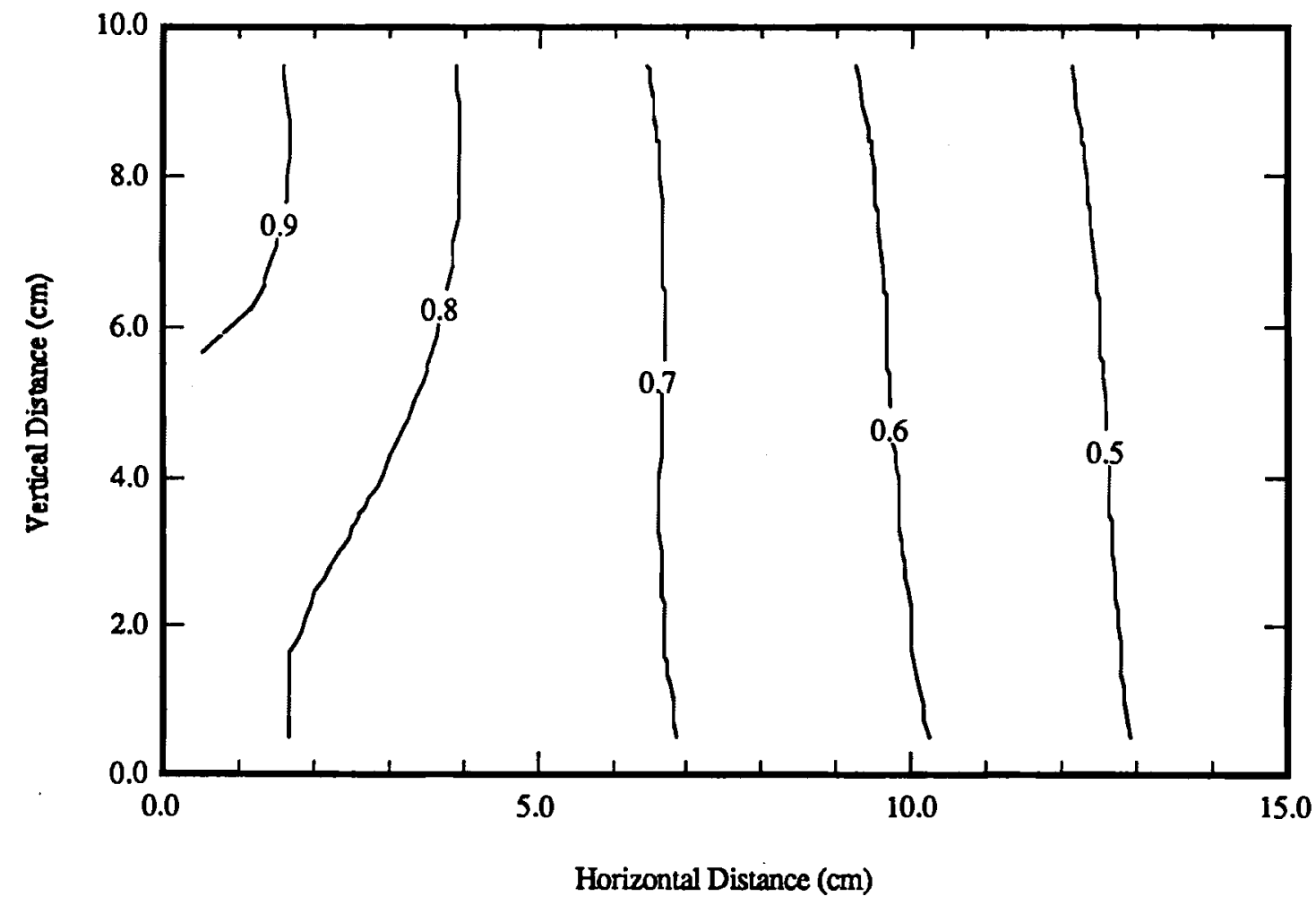

Figure 8.7. Liquid Saturation Contours at 0.508 Day for Two-Dimensional Infiltration Problem 
Exhibit 8.6. MSTS Input File for Two-Dimensional Infiltration Problem

\section{Line No. Input File}

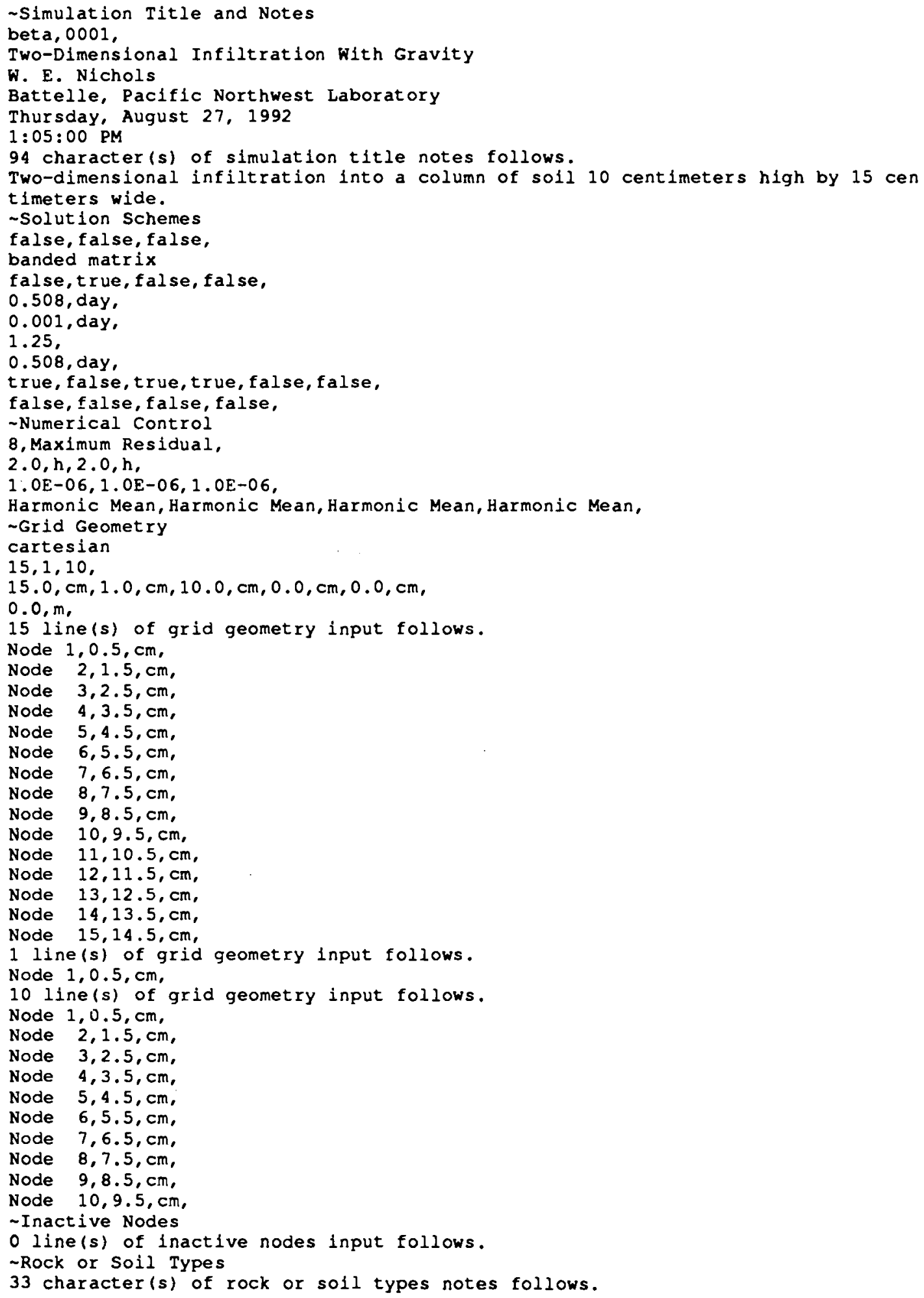




\section{Exhibit 8.6. (Contd)}

\section{Line No. Input File}

651 line(s) of rock or soil types input follows.

66 Homogeneous Sol1,1,15, 1, 1, 1,10,

67 Mechanical Properties

68 I line(s) of mechanical properties input follows.

69 Homogeneous Soll, 0.45, 0.45,0.30, ., , dependent, .,

70 - Hydraulic Properties

71 line(s) of hydraulic properties input follows.

72

Homogeneous Soil, $1.2 \mathrm{E}-14,1.2 \mathrm{E}-14,1.2 \mathrm{E}-14, \mathrm{~m}^{\wedge} 2, \ldots, \mathrm{m}^{\wedge} 2,1.9,1 / \mathrm{m}$,
- Thermal Properties

1 line(s) of thermal properties input follows.

Homogeneous Soll, independent, $1.0 e+5,1.0 e+5,1.0 e+5,1.0 e+5,1.0 e+5,1.0 e+5, \mathrm{~W} / \mathrm{m} \mathrm{K}$, $2385, \mathrm{~kg} / \mathrm{m}^{\wedge} 3,1030, \mathrm{~J} / \mathrm{kg} \mathrm{K}$,

- Species Properties

0 line(s) of species properties input follows.

-Soll Characteristics

1 Ine (s) of soll characteristics input follows.

Homogeneous Soil, Tabular, Table No. 1,

2 line(s) of capillary tables input follows.

$0.0,1.0, \mathrm{~m}$,

$1.0,0.333, \mathrm{~m}$,

- Iiquid Relative Permeability Properties

1 line(s) of liquid relative permeability input follows.

Homogeneous Soll, Tabular vs Liquid Saturation, Table No. 1 ,

3 line(s) of liquid permeability tables input follows.

0.0 .0 .0 .

$0.333,0.0$

$1.0,1.0$,

- Gas Relative Permeability Properties

1 line(s) of gas relative permeability input follows.

Homogeneous Soil, Tabular vs Liquid Saturation, Table No. 1,

2 line(s) of gas permeability tables input follows.

$0.0,1.0$,

$1.0,1.0$,

- Liquid Boundary Conditions

5 line(s) of liquid boundary conditions input follows.

X-Direction: West Surface,Dirichlet e Boundary,101276.06, Pa (absolute),998.32, $\mathrm{kg} / \mathrm{m} \wedge 3,1,1,1,1,7,7$,

100

X-Direction: West Surface,Dirichlet Boundary,101178.17,Pa (absolute),998.32, $\mathrm{kg} / \mathrm{m} \wedge 3,1,1,1,1,8,8$,

101 X-Direction: West Surface,Dirichlet B Boundary, 101080.28, Pa (absolute), 998.32, $\mathrm{kg} / \mathrm{m}^{\wedge} 3,1,1,1,1,9,9$,

X-Direction: West Surface,Dirichlet f Boundary,100982.39, Pa (absolute),998.32, $\mathrm{kg} / \mathrm{m} \backsim 3,1,1,1,1,10,10$,

X-Direction: East Surface, Dirlchlet E Boundary,92514.90,Pa (absolute),998.32, $\mathrm{kg} / \mathrm{m}^{\wedge} 3,15,15,1,1,1,10$,

- Gas Boundary Conditions

o line(s) of gas boundary conditions input. follows.

- Vapor Boundary Conditions

0 line(s) of vapor boundary conditions input follows.

-Energy Boundary Conditions

O line(s) of energy boundary conditions input follows.

-Species Boundary Conditions

o line(s) of species boundary conditions input follows.

- Initial Conditions

Liquid Saturation \& Gas Pressure,

2 line(s) of initial conditions input follows.

Gas Pressure, 101325.0, Pa (absolute),.,1/m, 1/m, 1/m, 1, 15, 1, 1, 1, 10,

Liquid Saturation, $0.3333, \ldots, 1 / \mathrm{m}, 1 / \mathrm{m}, 1 / \mathrm{m}, 1,15,1,1,1,10$,

-Sources \& Sinks

0 line(s) of sources sinks input follows.

- Liquid Pathline Trace

0 line(s) of liquid pathline trace input follows. 
Exhibit 8.6. (Contd)

\section{Line No. Input File}

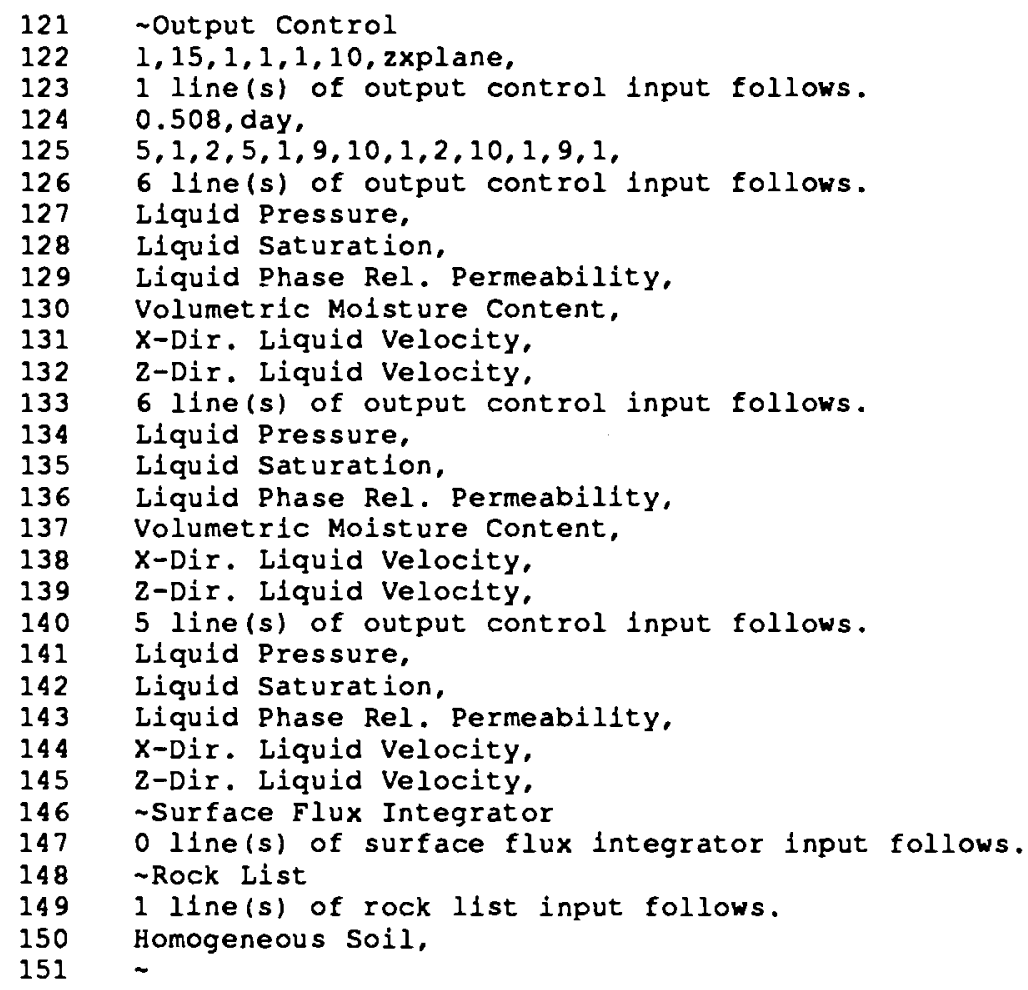




\subsection{One-Dimensional Two-Phase Infiltration}

Touma and Vauclin (1986) demonstrated, both experimentally and numerically, the effects of air flow on water infiltration in a 93.5-cm column of soil. Touma and Vauclin (1986) applied three types of boundary conditions to the top of the soil column: 1) positive time-constant liquid head, 2) positive time-constant liquid flux less than the saturated soil hydraulic conductivity, and 3) positive time-constant liquid flux greater than the saturated hydraulic conductivity. In this section, the positive time-constant liquid head (referred to as ponded infiltration) simulations of Touma and Vauclin (1986) are repeated using MSTS to demonstrate the impact of air flow on water infiltration rates. Two simulations are described: one for a column open to air flow at the base, and one closed at the base so that air must flow out the top as water infiltrates.

\subsubsection{Problem Domain and Input Parameters}

The problem domain is a column of soil of height $L=93.5 \mathrm{~cm}$ for which hydraulic properties were experimentally determined by Touma and Vauclin (1986). The experimental data for the relationship between capillary pressure head and water content were statistically fit by Touma and Vauclin (1986) with the van Genuchten analytical expression (van Genuchten 1980). No distinction was made between drainage and infiltration in this expression (i.e., hysteresis was not modeled). The relationships between soil-moisture tension and liquid saturation used in the MSTS simulation are shown in Figure 8.8a. The relationships between air and water relative permeability and liquid saturation were experimentally determined and are shown in Figure 8.8b.

The first case is the open column, which represents the traditional single-phase solution for water infiltration neglecting air-flow effects. The MSTS input file for this problem is provided in Exhibit 8.7. The Water mode (Section 2.4.1) is used in this solution. The upper boundary is specified as a Dirichlet condition equivalent to a constant hydraulic head of $2.3 \mathrm{~cm}$ while the lower boundary is specified as a Dirichlet condition equal to the initial condition (Exhibit 8.7, Liquid Boundary Conditions card, lines 157-160). Touma and Vauclin (1986) specified that the initial condition of the soil column was reached by draining the saturated column to the static equilibrium corresponding to a piezometric level at $120 \mathrm{~cm}$ below the surface (top) of the column. The initial hydrologic conditions are specified as a uniform gas pressure of 101,325 $\mathrm{Pa}$ and a liquid pressure that is computed directly from the van Genuchten relation for a zero hydraulic gradient (pressure head and elevation head equal throughout). Examine the actual input card (Exhibit 8.7, Initial Conditions card, lines 169-173) to see how the input condition is specified using the gradient feature of the Initial Conditions card. 


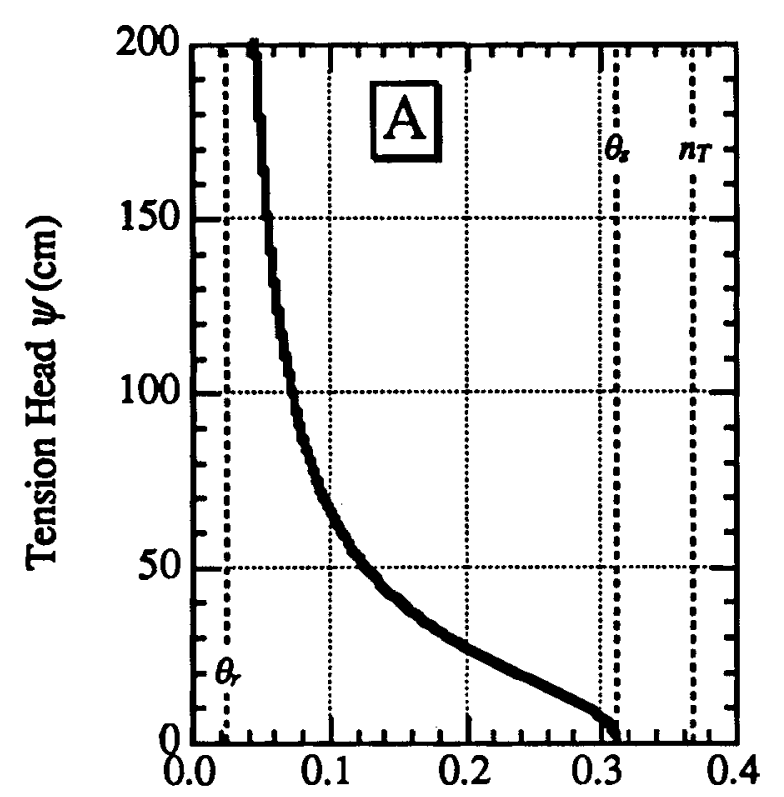

Volumetric Moisture Content $\theta\left(\mathrm{cm}^{3} / \mathrm{cm}^{3}\right)$

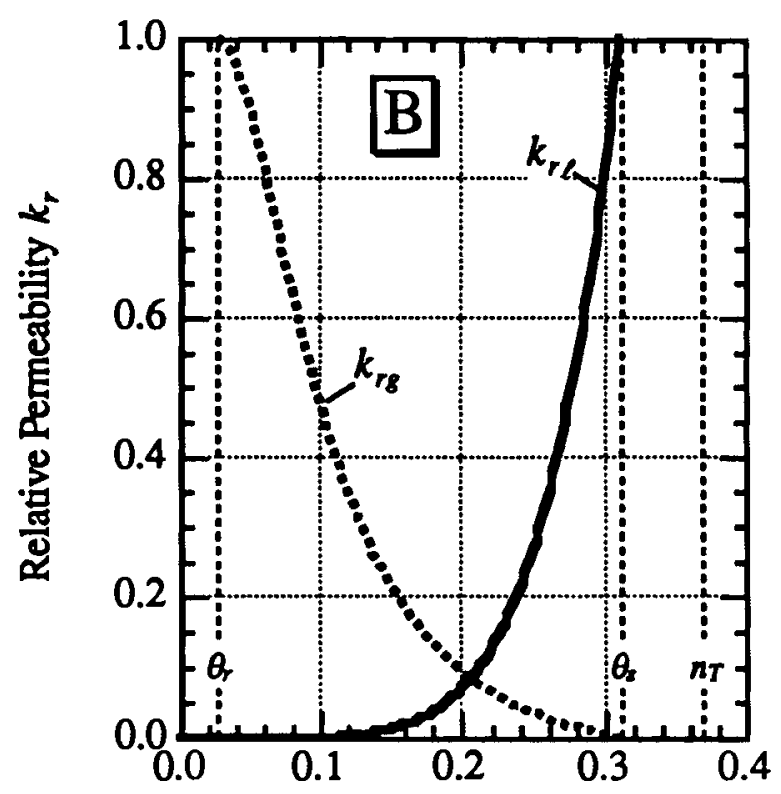

Volumetric Moisture Content $\theta\left(\mathrm{cm}^{3} / \mathrm{cm}^{3}\right)$

Figure 8.8. Moisture-Retention and Relative-Permeability Functions for the One-Dimensional Two-Phase Infiltration Problem: A) Relationship Between Volumetric Moisture Content and Tension Head, B) Relationship Between Volumetric Moisture Content and Liquid and Gas Relative Permeabilities

The second simulation includes the effects of air flow in the column. The Water-Air mode (Section 2.4.3) is used and the gas boundary conditions are defined to address this issue. Exhibit 8.8 shows the MSTS input file for this simulation. A longer time is simulated than for the open column case ( 1.2 hour rather than 0.4 hour) because the inclusion of air flow slows the wetting front greatly, as reported by Touma and Vauclin (1986). Touma and Vauclin also reported that the air-entry pressure was estimated to be $14 \mathrm{~cm}$ for this problem. Hence, the gas pressure at the upper boundary is specified as the pressure equivalent of the liquid pressure boundary $(2.3 \mathrm{~cm})$ plus the air-entry pressure $(14.0 \mathrm{~cm})$, or 101,550.25 Pa (Exhibit 8.8, Gas Boundary Conditions card, lines 161-164).

\subsubsection{Simulation Results}

The water content profiles for the open-column case, which represents traditional single-phase infiltration models, are depicted in Figure 8.9. The water content profiles for the bounded-column case are depicted in Figure 8.10. Comparing the two figures, we observe as Touma and Vauclin (1986) reported that the rate of advance of the wetting front is drastically reduced when air cannot escape. Notice also the reduction in the maximum saturation of the water content profiles 


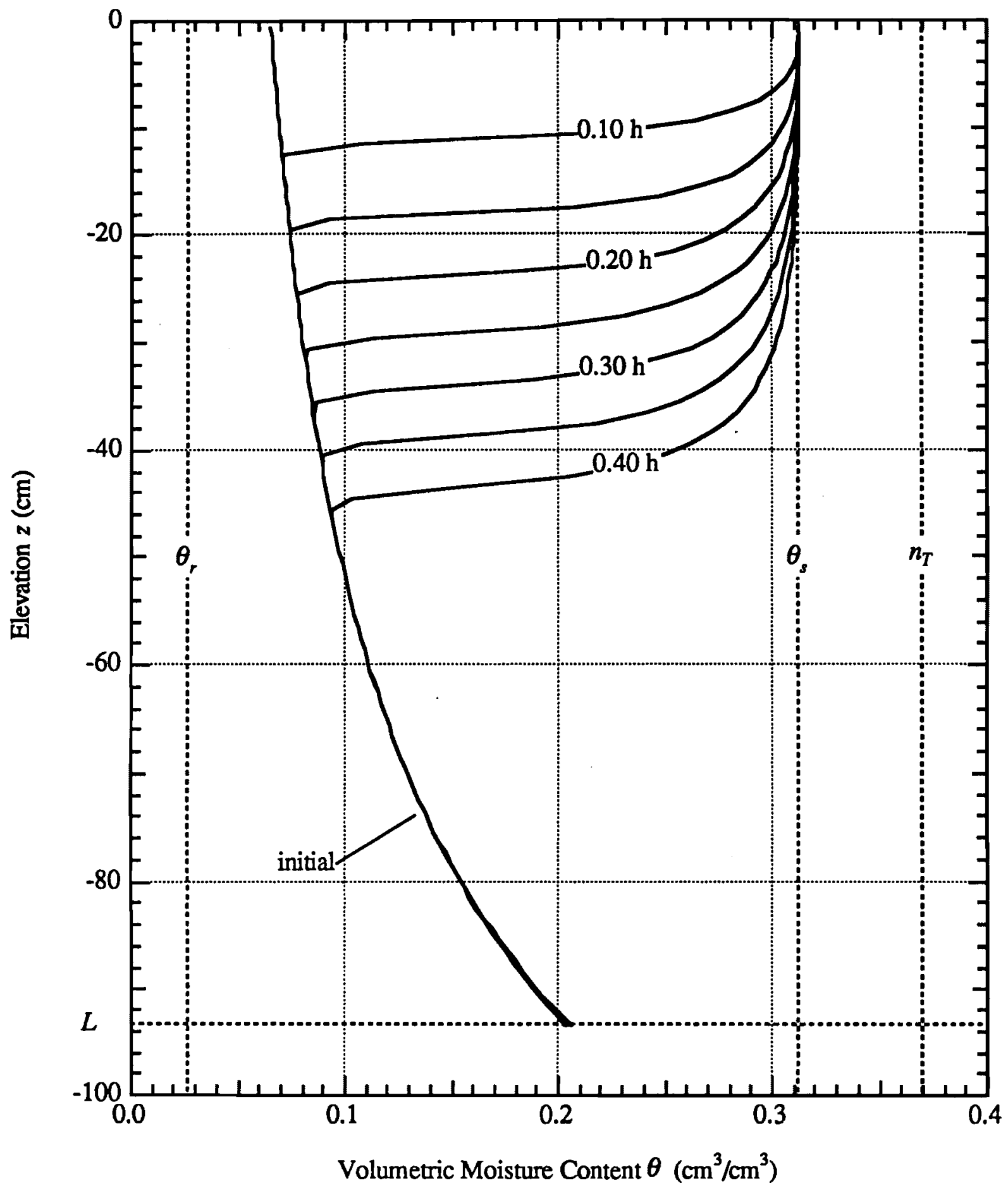

Figure 8.9. Water Content Profiles Predicted by MSTS for Ponded Infiltration in the OneDimensional, Two-Phase Infiltration Problem: Open Column 


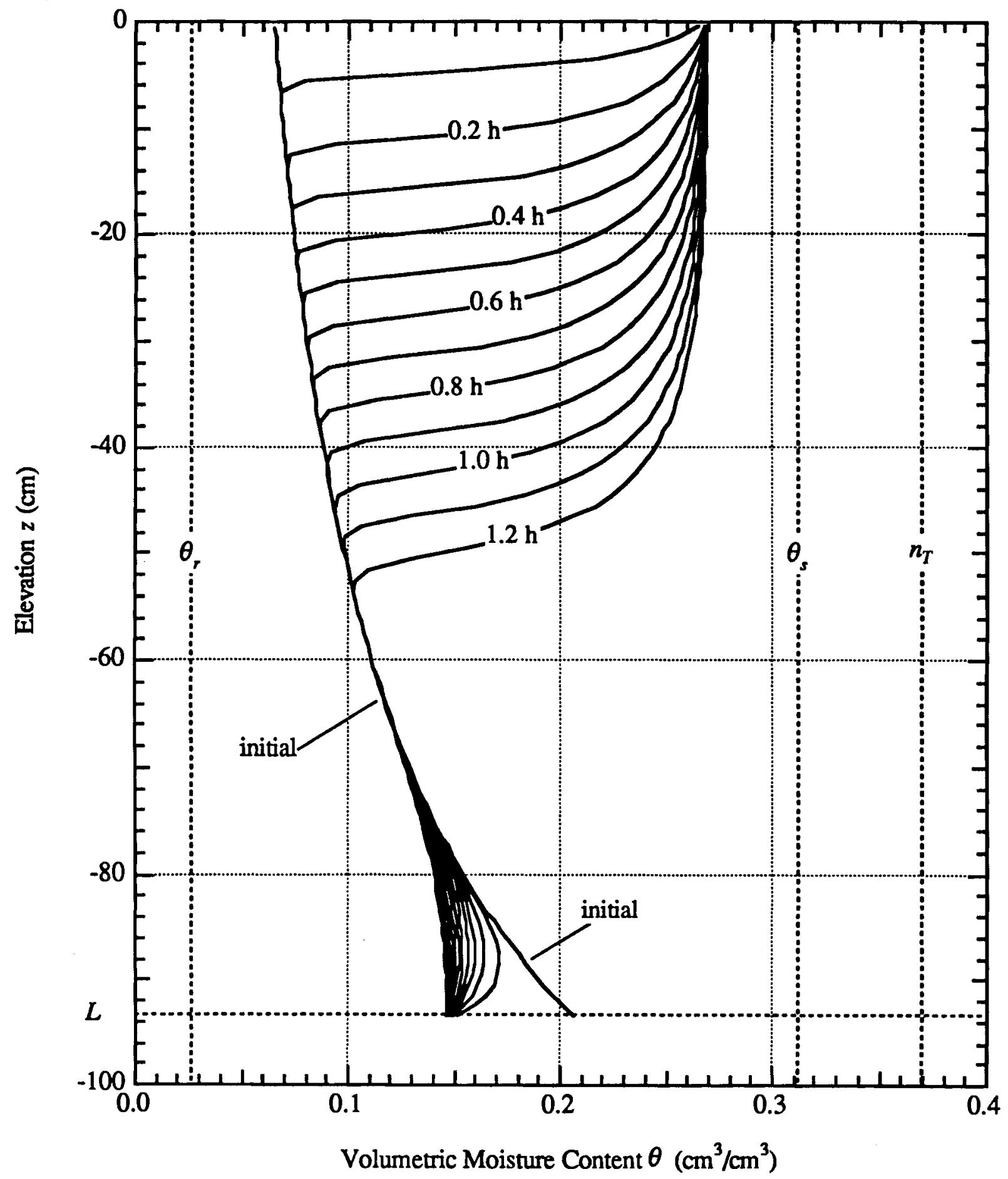

Figure 8.10. Water Content Profiles Predicted by MSTS for Ponded Infiltration in the OneDimensional, Two-Phase Infiltration Problem: Bounded Column 
from $0.312 \mathrm{~cm}^{3} / \mathrm{cm}^{3}$ in the open-column case to approximately $0.27 \mathrm{~cm}^{3} / \mathrm{cm}^{3}$ in the boundedcolumn case, an effect caused by the flow of escaping air. Finally, we note that the wetting fronts shown in Figure 8.10 for the bounded-column case are less steep than for the open-column case shown in Figure 8.9. 


\section{Exhibit 8.7. MSTS Input File for Two-Phase Infiltration Problem, Open Column}

Line No. Input File

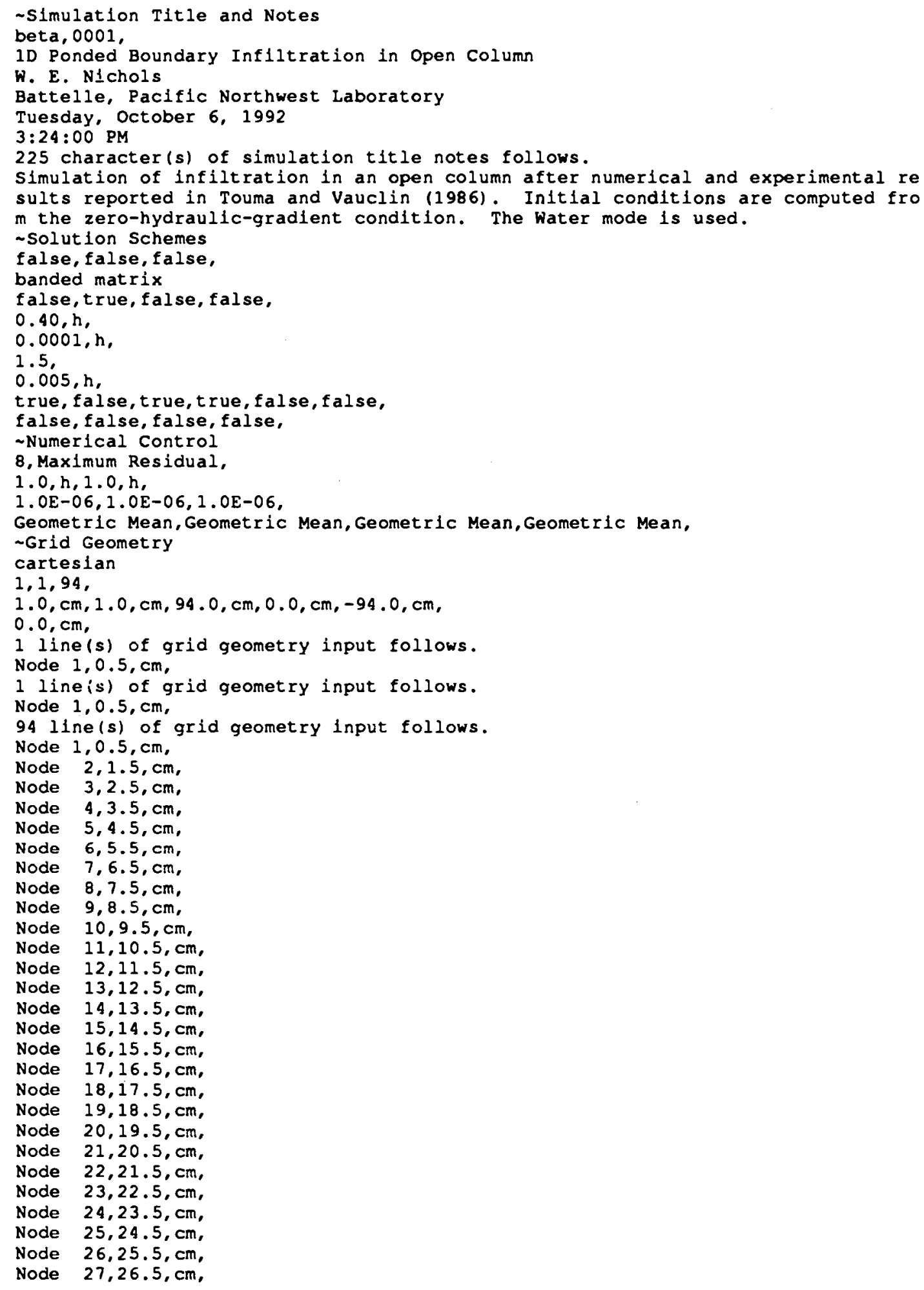


Exhibit 8.7. (Contd)

Line No Input File

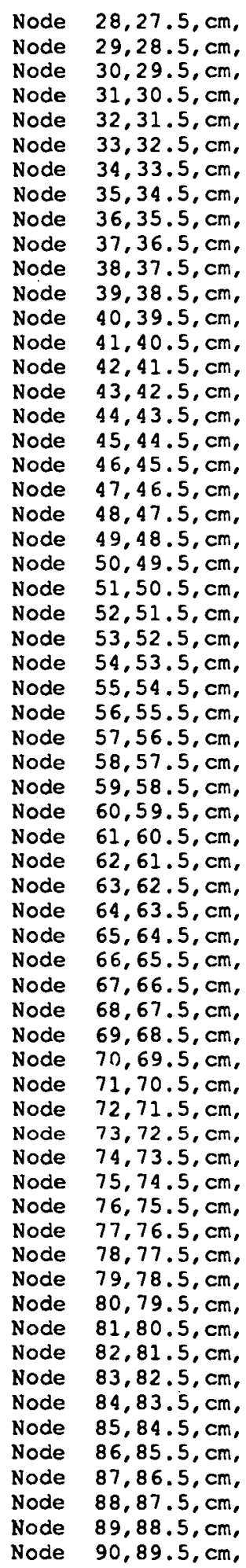




\section{Exhibit 8.7. (Contd)}

\section{Line No. Input File}

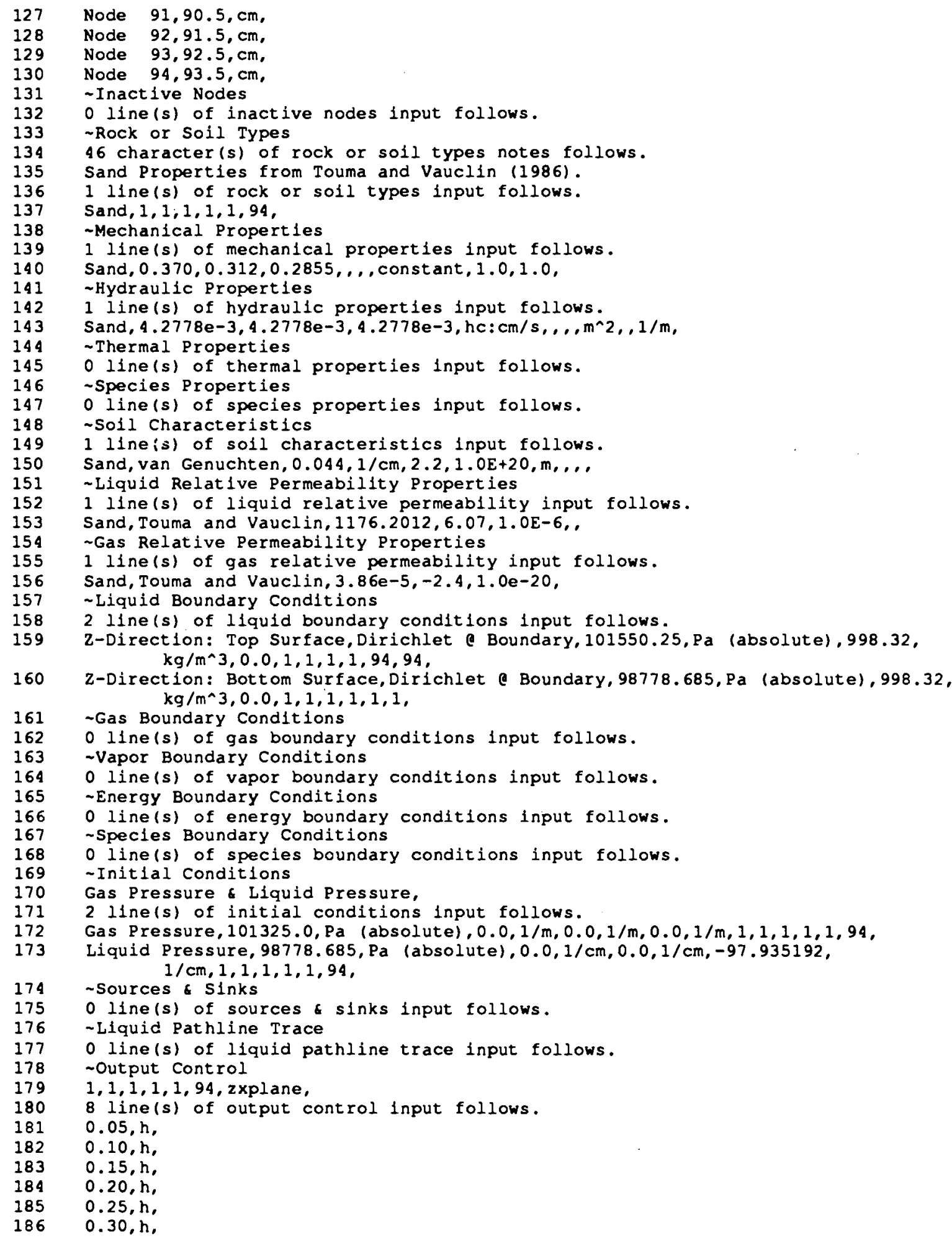


Exhibit 8.7. (Contd)

Line No. Input File

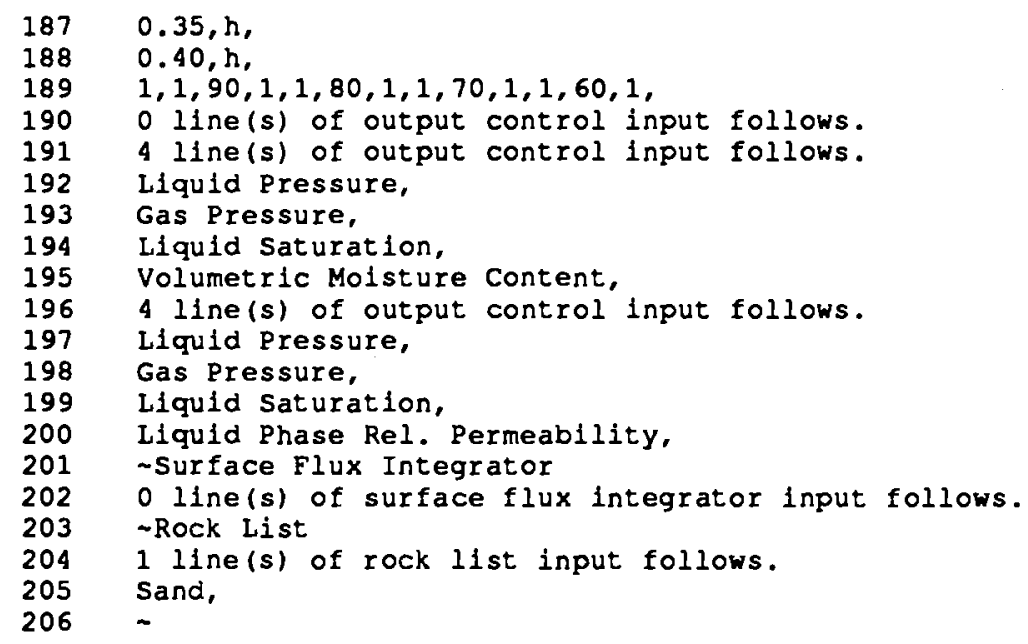




\section{Exhibit 8.8. MSTS Input File for Two-Phase Infiltration Problem, Bounded Column}

\section{Line No. Input File}

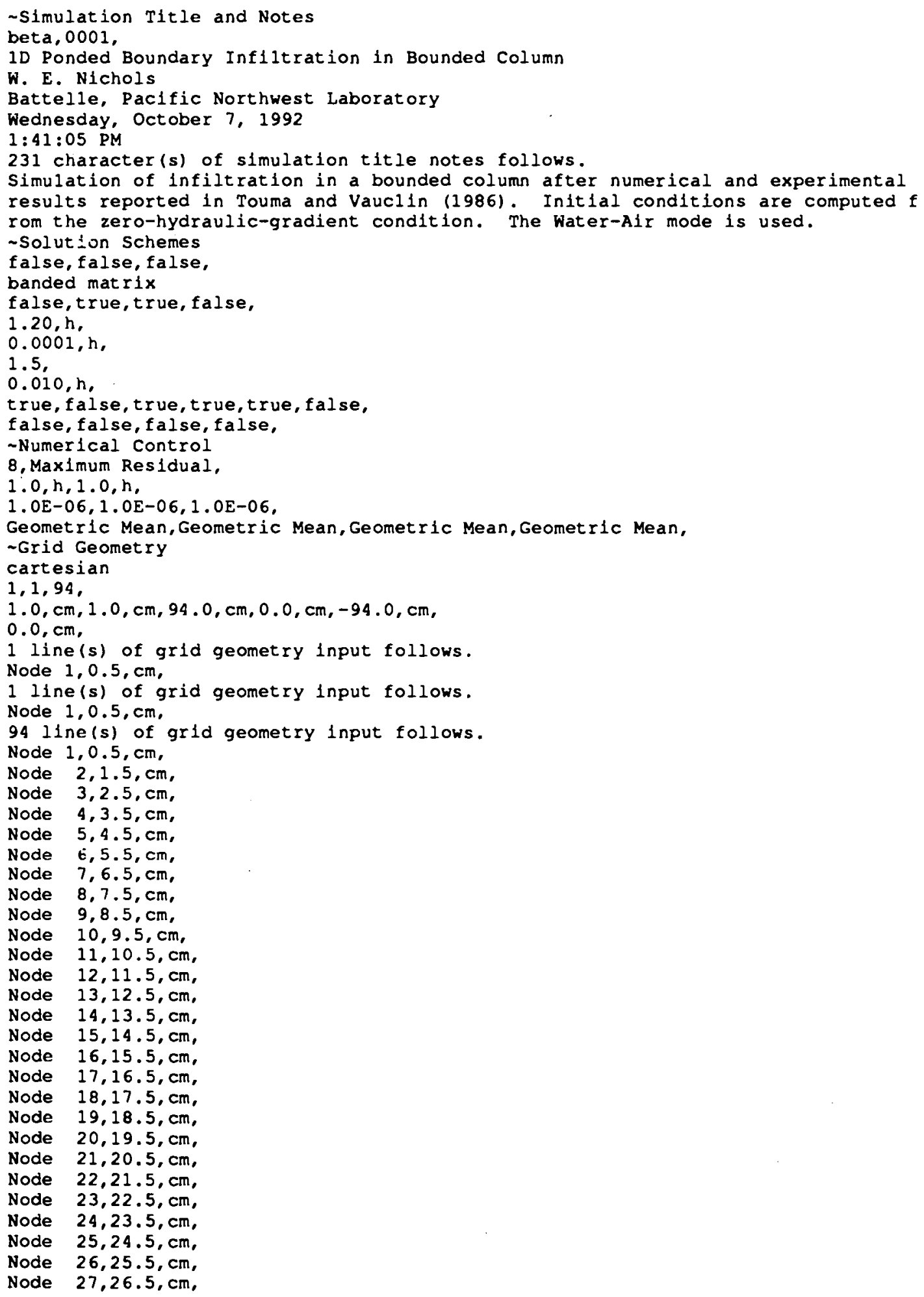


Exhibit 8.8. (Contd)

\section{Line No. Input File}

\begin{tabular}{|c|c|c|}
\hline 64 & Node & $28,27.5, \mathrm{~cm}$, \\
\hline 65 & Node & $29,28,5, \mathrm{~cm}$, \\
\hline 66 & Node & $30,29.5, \mathrm{~cm}$, \\
\hline 67 & Node & $31,30.5$ \\
\hline 68 & Node & $32,31,5, \mathrm{~cm}$, \\
\hline 69 & Node & $33,32,5, \mathrm{~cm}$, \\
\hline 70 & Node & $34,33,5, \mathrm{~cm}$, \\
\hline 71 & Node & $35,34,5, \mathrm{~cm}$, \\
\hline 72 & Node & $5, \mathrm{~cm}$, \\
\hline 73 & Node & $37,36,5, \mathrm{~cm}$, \\
\hline 74 & Node & $38,37,5, \mathrm{~cm}$, \\
\hline 75 & Node & $39,38,5, \mathrm{~cm}$, \\
\hline 76 & Node & $40,39.5, \mathrm{~cm}$, \\
\hline 77 & Node & $41,40.5, \mathrm{~cm}$, \\
\hline 78 & Node & $42,41,5, \mathrm{~cm}$, \\
\hline 79 & Node & $43,42.5, \mathrm{~cm}$ \\
\hline 80 & Node & $5, \mathrm{~cm}$, \\
\hline 81 & Node & $45,44,5, \mathrm{~cm}$ \\
\hline 82 & Node & $46,45,5, \mathrm{~cm}$ \\
\hline 83 & Node & $47,46.5, \mathrm{~cm}$ \\
\hline 84 & Node & $48,47.5, \mathrm{~cm}$, \\
\hline 85 & Node & $49,48.5, \mathrm{~cm}$ \\
\hline 86 & Node & $50,49.5, \mathrm{~cm}$, \\
\hline 87 & Node & $51,50.5, \mathrm{~cm}$, \\
\hline 88 & Node & $52,51.5, \mathrm{~cm}$, \\
\hline 89 & Node & $53,52.5, \mathrm{~cm}$, \\
\hline 90 & Node & $54,53.5, \mathrm{~cm}$, \\
\hline 91 & Node & $55,54.5, \mathrm{~cm}$, \\
\hline 92 & Node & $56,55.5, \mathrm{~cm}$, \\
\hline 93 & Node & $57,56.5, \mathrm{~cm}$, \\
\hline 94 & Node & $58,57.5, \mathrm{~cm}$, \\
\hline 95 & Node & $8.5, \mathrm{~cm}$, \\
\hline 96 & Node & $60,59.5, \mathrm{~cm}$. \\
\hline 97 & Node & $61,60.5, \mathrm{~cm}$, \\
\hline 98 & Node & $62,61,5, \mathrm{~cm}$, \\
\hline 99 & Node & $63,62.5, \mathrm{~cm}$, \\
\hline 100 & Node & $64,63.5, \mathrm{~cm}$ \\
\hline 101 & Node & $65,64.5, \mathrm{~cm}$ \\
\hline 102 & Node & 66,65 \\
\hline 103 & Node & $67,66,5, \mathrm{~cm}$ \\
\hline 104 & Node & $68,67.5, \mathrm{~cm}$ \\
\hline 105 & Node & $69,68.5, \mathrm{~cm}$, \\
\hline 106 & Node & 70,69 \\
\hline 107 & Node & $71,70.5, \mathrm{~cm}$ \\
\hline 108 & Node & 72,71 \\
\hline 109 & Node & $73,72.5, \mathrm{~cm}$ \\
\hline 110 & Node & 74.73 \\
\hline 111 & Node & 75,74 \\
\hline 112 & Node & $\mathrm{cm}$, \\
\hline 113 & Node & 77,76 \\
\hline 114 & Node & 79,77 \\
\hline 115 & Node & 79,78 \\
\hline 116 & Node & $80,79.5, \mathrm{~cm}$, \\
\hline 117 & Node & $81,80.5, \mathrm{~cm}$, \\
\hline 118 & Node & $82,81,5, \mathrm{~cm}$, \\
\hline 119 & Node & $5, \mathrm{~cm}$, \\
\hline 120 & Node & 84,83 \\
\hline 121 & Node & 85,8 \\
\hline 122 & Node & $86,85,5, \mathrm{~cm}$, \\
\hline 123 & Node & $87,86.5, \mathrm{~cm}$, \\
\hline 124 & Node & $88,87.5, \mathrm{~cm}$, \\
\hline 125 & Node & $89,88.5, \mathrm{~cm}$ \\
\hline 126 & Node & $90,89.5, \mathrm{~cm}$, \\
\hline
\end{tabular}




\section{Exhibit 8.8. (Contd)}

\section{Line No. Input File}

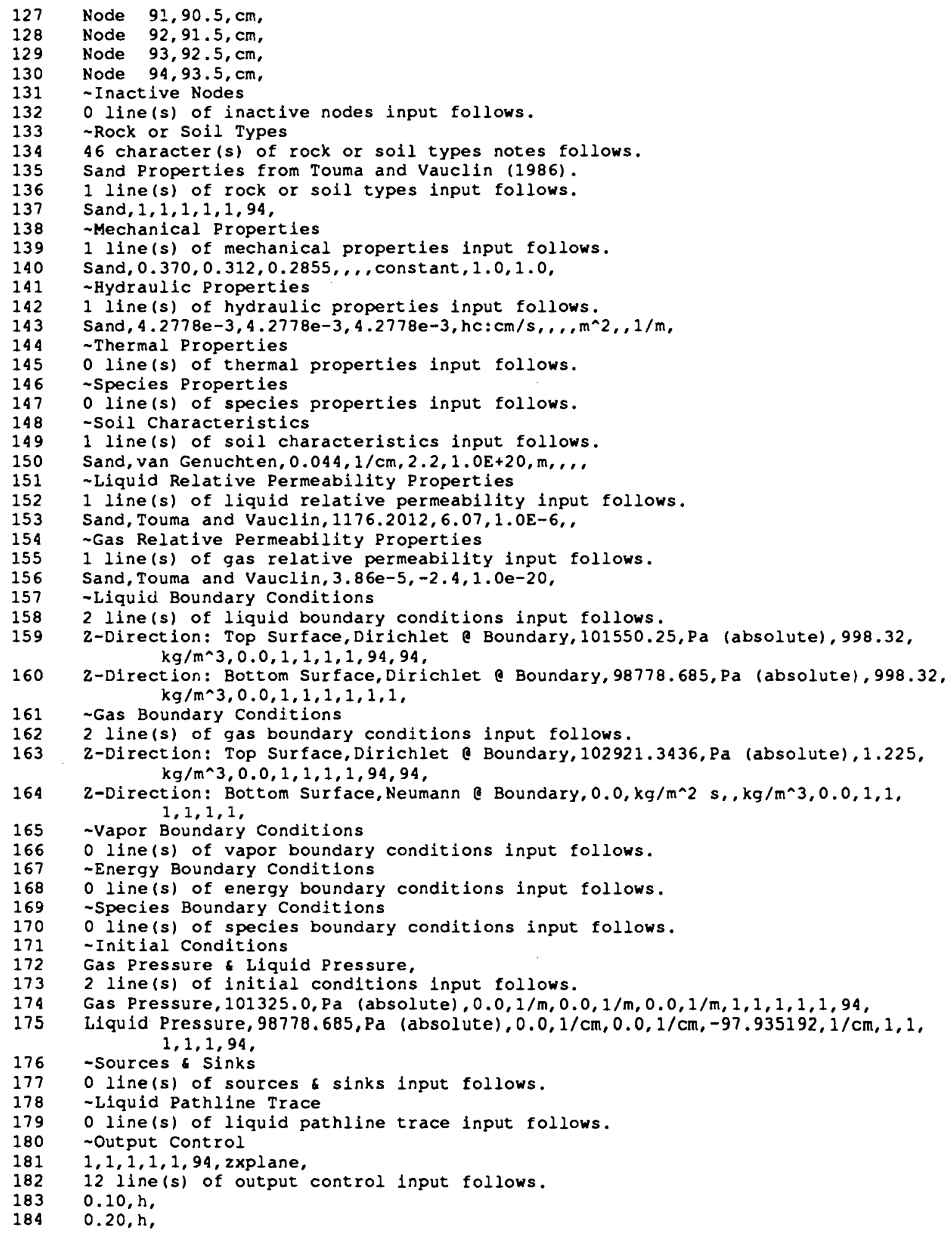




\section{Exhibit 8.8. (Contd)}

Line No Input File

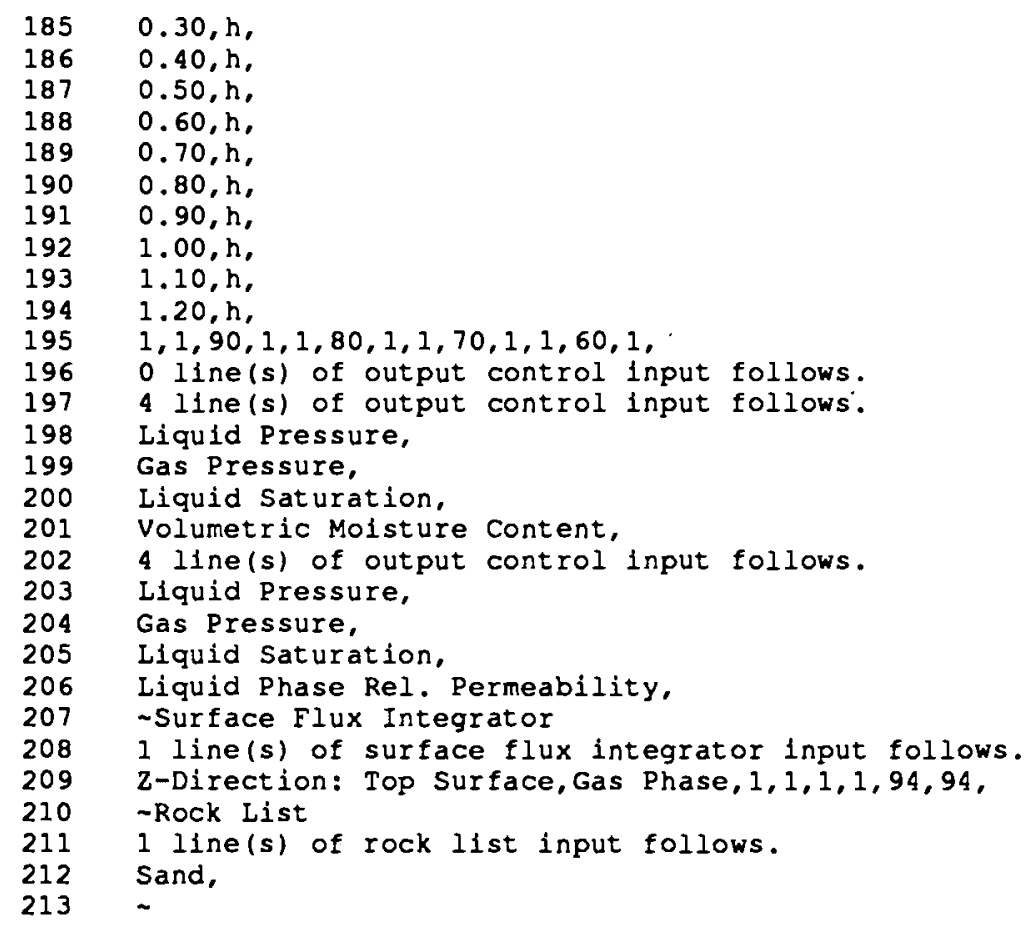




\subsection{One-Dimensional Heat Pipe}

The heat pipe problem involves a one-dimensional horizontal layer of porous medium subjected to a heat flux on one end and is characterized by high heat transfer rates and countercurrent flow of liquid and gas phases. Both phases are composed of water and air components (e.g., liquid water and dissolved air for the liquid phase, and air and water vapor for the gas phase). The specifics of the subject problem were described and solved by Udell and Fitch (1985) for constant physical properties at steady state. The Udell and Fitch model used a twocomponent gas phase made up of nitrogen and water vapor. A schematic of the problem proposed by Udell and Fitch is shown in Figure 8.11. The problem geometry consists of a tube, with an adiabatic wall, filled with a partially saturated porous medium. The right end of the tube is closed and subjected to a heat flux of $100 \mathrm{~W} / \mathrm{m}^{2}$. Conditions at the left end of the tube are maintained constant at total saturation, $70^{\circ} \mathrm{C}$, and $101,330 \mathrm{~Pa}$ (absolute) gas pressure. Initial conditions within the tube are 0.7 liquid saturation, $70^{\circ} \mathrm{C}$, and $101,330 \mathrm{~Pa}$ (absolute) gas pressure. The problem involves solving the transient response of the heat pipe from the initial conditions to steady-state conditions. The problem was selected to test the simulator's capability to model coupled two-phase flow, heat transport, and evaporation and condensation processes.

\subsubsection{Problem Parameters and Input File}

The soil-moisture-retention characteristics and saturation-relative-permeability functions for the porous media were described as shown in Equations (8.8) through (8.12). The Udell and Fitch model used a constant water-air surface tension value of $0.05878 \mathrm{~N} / \mathrm{m}$, instead of the

Adiabatic/Zero-Flux Horizontal Boundaries

\begin{tabular}{ll|}
\hline Initial Conditions: & Liquid Saturation $s_{\ell}=0.5$ \\
& Gas Pressure $P_{g}=101,330 \mathrm{~Pa}$ \\
& Temperature $T^{\circ}=70^{\circ} \mathrm{C}$ \\
\hline & $2.25 \mathrm{~m}$
\end{tabular}

Liquid Saturation $s_{\ell}=1.0$

Gas Pressure $P_{g}=101,330 \mathrm{~Pa}$

Temperature $\mathrm{T}=70.0^{\circ} \mathrm{C}$
Liquid Flux $\dot{Q}_{\ell}=0.0$

Gas Flux $\dot{Q}_{g}=0.0$

Heat Flux $\dot{Q}_{e}=-100.0 \mathrm{~W} / \mathrm{m}^{2}$

Figure 8.11. Schematic Describing the Heat Pipe Problem 
temperature dependent model shown in Equation (8.9). The thermal conductivity of the porous medium was considered a function of the effective liquid saturation as shown in Equation (8.12). Properties for density, viscosity, heat of vaporization, and molecular diffusion coefficient were computed in MSTS as functions of local temperature and pressures; for the Udell and Fitch model, these parameters were considered constants. These constants, and other physical properties required to define the heat pipe problem, according to Udell and Fitch, are listed in Table 8.6. The input file for this problem is shown in Exhibit 8.9.

$$
\begin{aligned}
& P_{c a p}=P_{g}-P_{\ell}=\sigma^{\alpha_{w}}\left(\frac{n_{D}}{\tilde{k}}\right)^{\frac{1}{2}}\left[1.417\left(1-s_{\ell}^{*}\right)-2.12\left(1-s_{\ell}^{*}\right)^{2}+1.263\left(1-s_{\ell}^{*}\right)^{3}\right] \\
& \sigma^{a w}=0.2358\left[1-0.625 \frac{(374.15-T)}{647.3}\right]\left[\frac{(374.15-T)}{647.3}\right]^{1.256} \\
& k_{r \ell}=\left(s_{\ell}^{*}\right)^{3} \\
& k_{r g}=\left(1-s_{\ell}^{*}\right)^{3} \\
& \tilde{k}_{e}=k_{d r y}+\sqrt{s_{\ell}^{*}}\left(k_{w e t}-k_{d r y}\right) \\
& \text { where } k_{d r y}=0.582 \frac{\mathrm{W}}{\mathrm{m} \mathrm{K}} \text { and } k_{\text {wet }}=1.130 \frac{\mathrm{W}}{\mathrm{m} \mathrm{K}}
\end{aligned}
$$


Table 8.6. Heat Pipe Problem Physical Properties

\begin{tabular}{|c|c|c|}
\hline Property Description & Symbol & Property Value \\
\hline Dry-rock thermal conductivity & $k_{d r y}$ & $0.582 \mathrm{~W} / \mathrm{m} \mathrm{K}$ \\
\hline Wet-rock thermal conductivity & $k_{\text {wet }}$ & $1.130 \mathrm{~W} / \mathrm{m} \mathrm{K}$ \\
\hline Gas density & $\rho_{g}$ & $0.960 \mathrm{~kg} / \mathrm{m}^{3}$ \\
\hline Liquid density & $\rho_{\ell}$ & $958.4 \mathrm{~kg} / \mathrm{m}^{3}$ \\
\hline Gas kinematic viscosity & $v_{8}$ & $21.0 \times 10-6 \mathrm{~m}^{2} / \mathrm{s}$ \\
\hline Liquid kinematic viscosity & $v e$ & $0.3066 \times 10^{-6} \mathrm{~m}^{2} / \mathrm{s}$ \\
\hline Heat of vaporization & $h_{\text {vap }}$ & $2.257 \times 106 \mathrm{~J} / \mathrm{kg}$ \\
\hline Intrinsic permeability & $k$ & $1.0 \times 10^{-12} \mathrm{~m}^{2}$ \\
\hline Surface tension & $\sigma$ & $0.05878 \mathrm{~N} / \mathrm{m}$ \\
\hline Molecular diffusion coefficient & $D_{g}^{a w}$ & $26.0 \times 10-6 \mathrm{~m} 2 / \mathrm{s}$ \\
\hline Nitrogen gas constant & $R$ & $461.52 \mathrm{~J} / \mathrm{kg} \mathrm{mol} \mathrm{K}$ \\
\hline Diffusive porosity & $n_{D}$ & 0.4 \\
\hline Gas tortuosity & $\tau_{g}$ & 0.5 \\
\hline Heat flux & $\dot{Q}_{e}$ & $100.0 \mathrm{~W} / \mathrm{m}^{2}$ \\
\hline
\end{tabular}

\subsubsection{Simulation Results}

The transient simulation begins by imbibing liquid water from the saturated boundary into the porous medium and increasing the temperature of the porous medium near the heat flux boundary. Liquid saturations near the heat flux boundary initially decrease because of the increased gas pressures. This initial decrease is followed by a short period of liquid saturation increase as the imbibed water from the saturated boundary reaches the other end of the pipe. The liquid saturations near the heat flux boundary then decrease to residual saturation levels as the temperature and pressures increase. The steady-state solution is characterized by three distinct regions within the heat pipe, as shown in Figures 8.12 through 8.14. Starting from the saturated boundary (left-hand side), the first region spans the distance from 0.0 to $0.5 \mathrm{~m}$. This region is characterized by sharp gradients in temperature and air mole fractions as shown in Figures 8.12 and 8.13. Both 


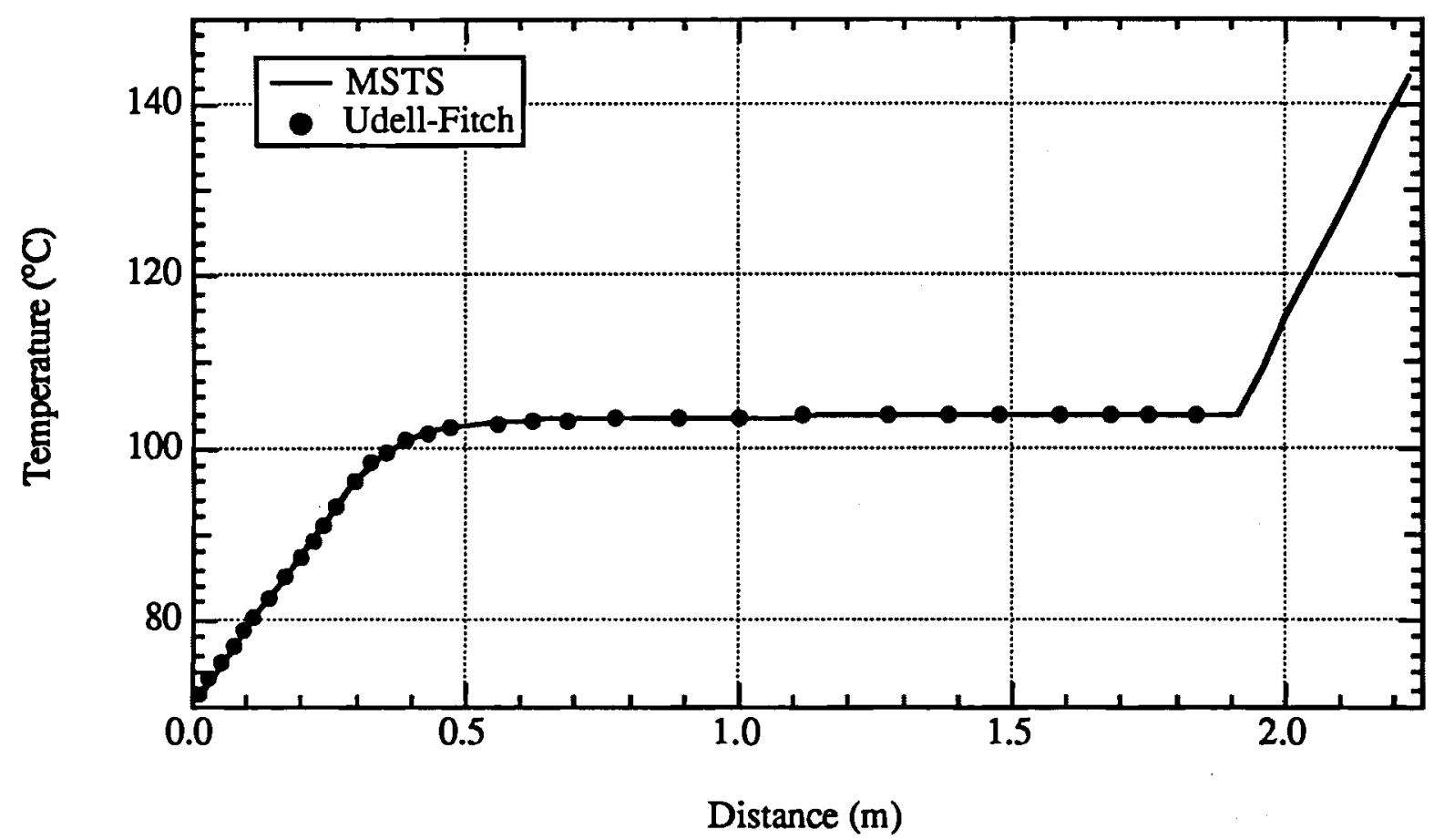

Figure 8.12. Steady-State Temperature Profiles for Heat Pipe Problem

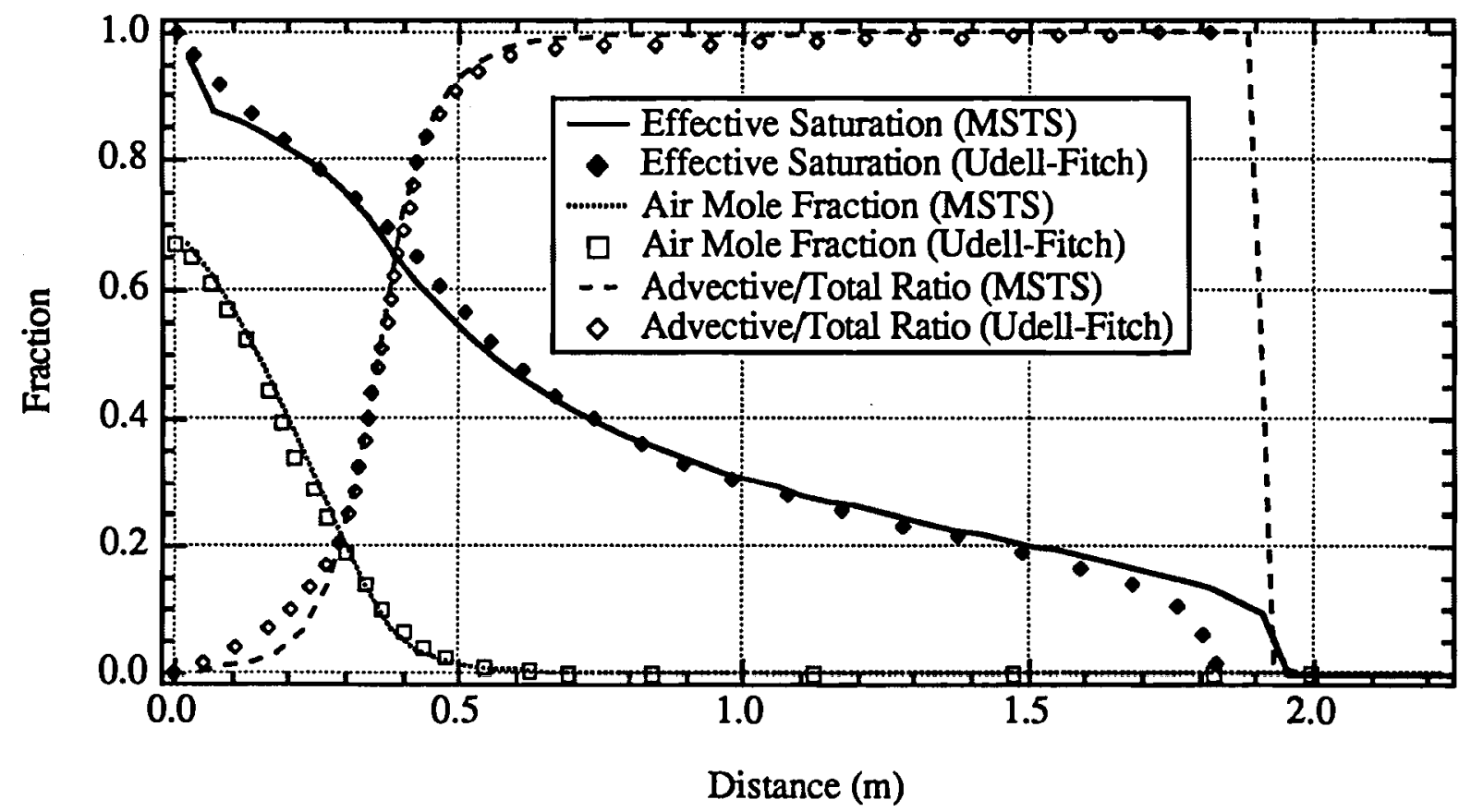

Figure 8.13. Steady-State Saturation, Air Mole Fraction, and Advective/Total Ratio Profiles for Heat Pipe Problem 


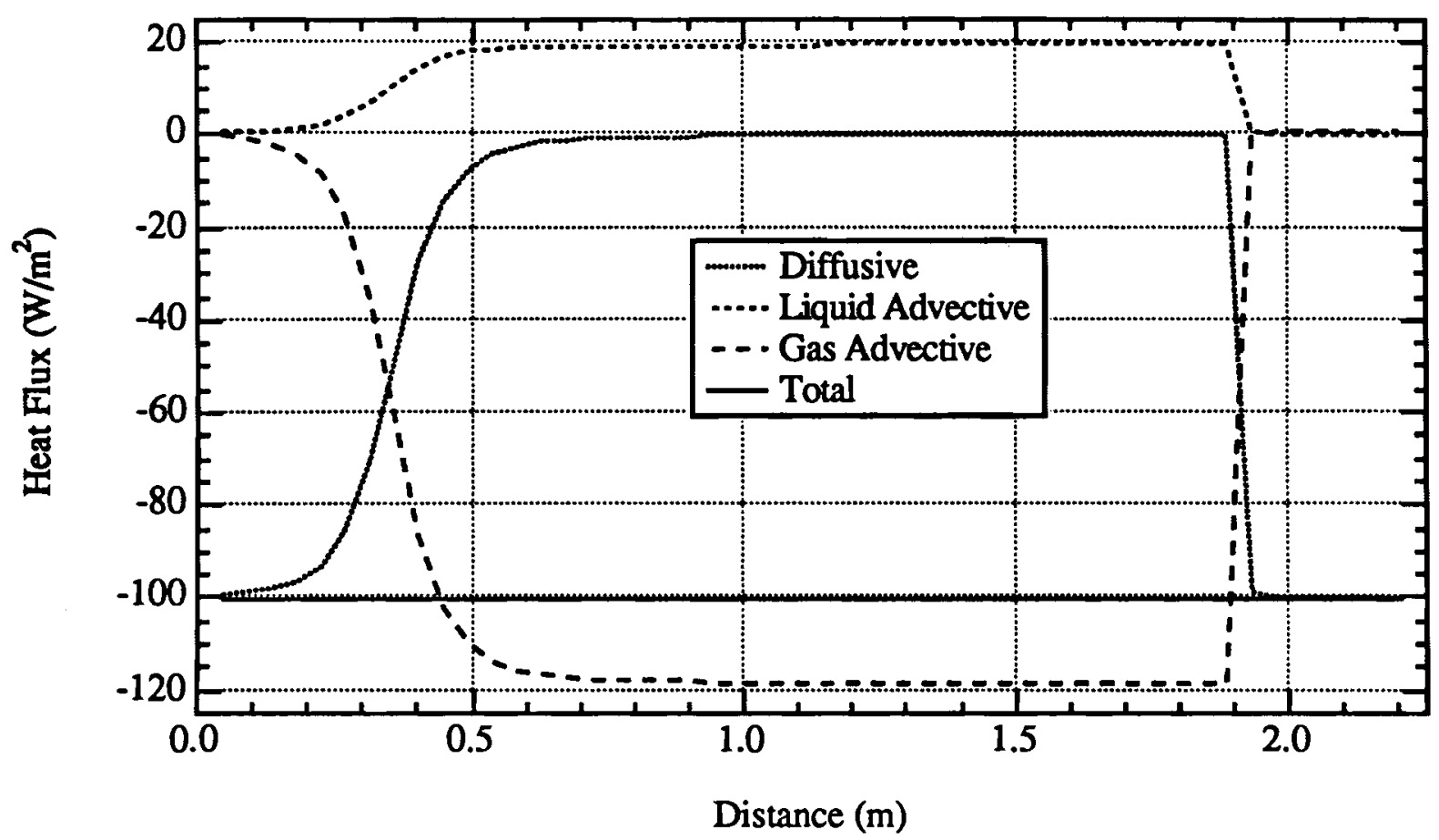

Figure 8.14. Steady-State Heat Transport Modes for Heat Pipe Problem

diffusive and advective heat transport mechanisms contribute to the overall heat transport. The second region spans from $0.5 \mathrm{~m}$ to $2.0 \mathrm{~m}$, and is characterized by strong countercurrent gas- and liquid-phase flow and near-isothermal conditions. The gas phase flows toward the left end of the pipe and is composed primarily of pure water vapor. The liquid phase returns the condensed water to the desaturated region at the left end of the pipe. Heat transport in this region of the pipe is dominated by advection, as indicated by the near-isothermal conditions. The third region of the heat pipe spans from $2.0 \mathrm{~m}$ to $2.25 \mathrm{~m}$, and is characterized by desaturated conditions. Heat transport in this region of the pipe is dominated by conduction. The MSTS results agree well with those computed by Udell and Fitch using a semi-analytical model with constant physical properties. 


\section{Exhibit 8.9. MSTS Input File for Heat Pipe Problem}

Line No. Input File

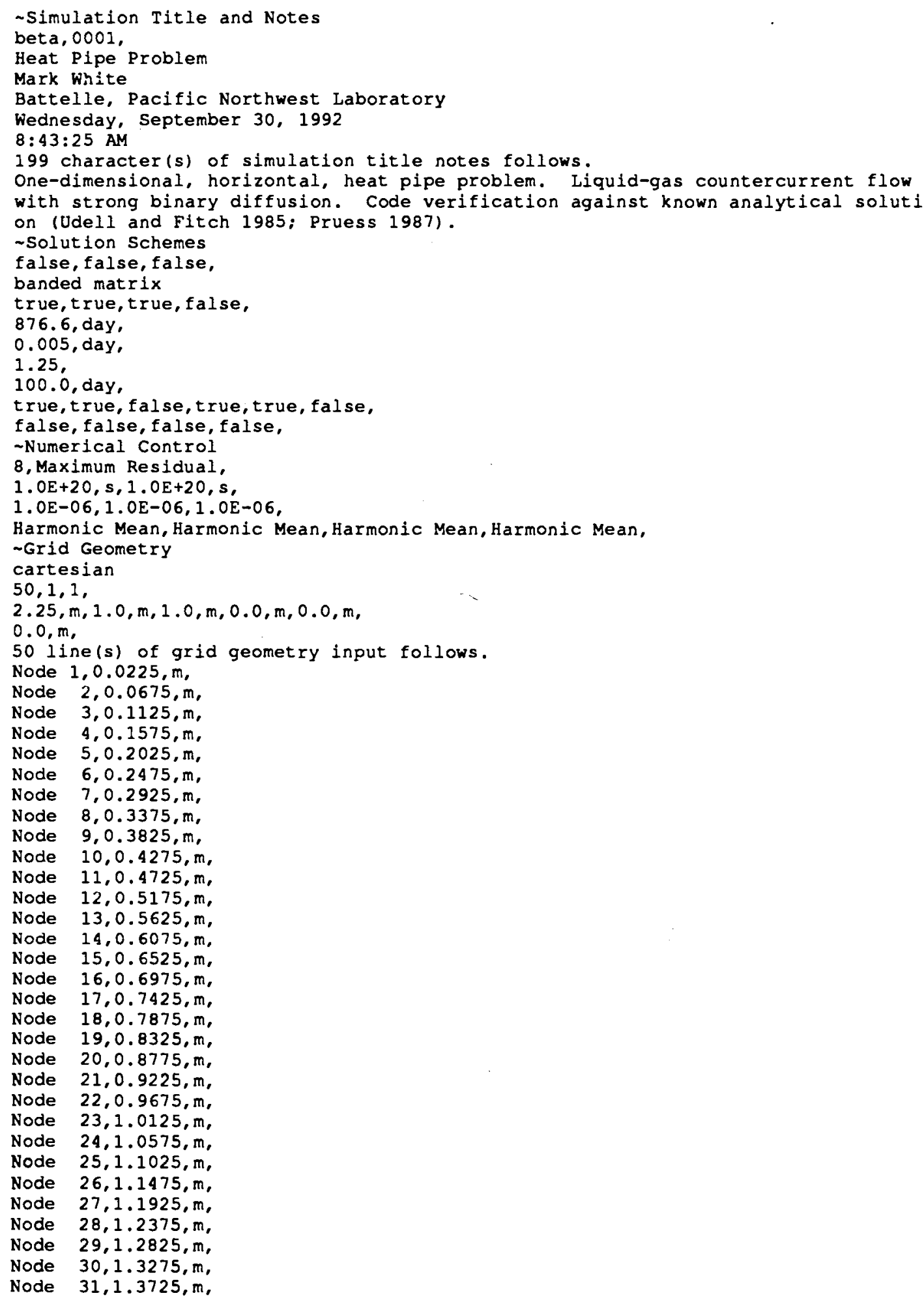


Exhibit 8.9. (Contd)

\section{Line No. Input File}

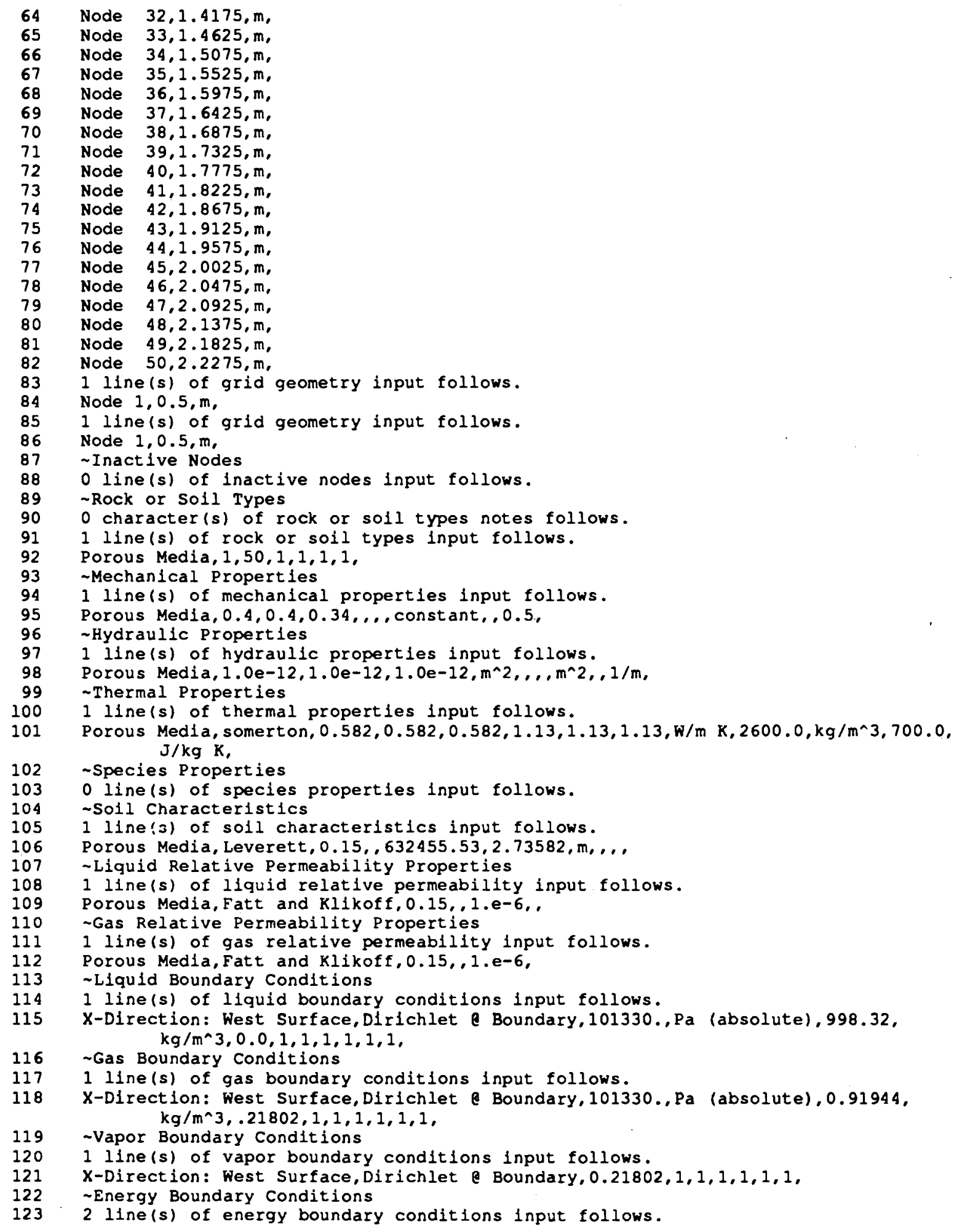




\section{Exhibit 8.9. (Contd)}

\section{Line No. Input File}

124

125

126

127

128

129

130

131

132

133

134

135

136

137

138

139

140

141

142

143

144

145

146

147

148

149

150

151

152

153

154

155

156

157

158

159

160

161

162

163

164

X-Direction: West Surface,Dirichlet Boundary, 70, Cels 1 us, $2.864 \mathrm{e}+8, \mathrm{~J} / \mathrm{m} \wedge 3$, $5.2098 \mathrm{e}+5, \mathrm{~J} / \mathrm{m}^{\wedge} 3,1,1,1,1,1,1$, $\mathrm{X}$-Direction: East Surface, Neumann B Boundary, $-100, \mathrm{~W} / \mathrm{m}^{\wedge} 2, \mathrm{~J} / \mathrm{m}^{\wedge} 3, \mathrm{~J} / \mathrm{m}^{\wedge} 3,50,50$, $1,1,1,1$,

- Species Boundary Conditions

o line(s) of species boundary conditions input follows.

Initial Conditions

Liquid Saturation \& Gas Pressure,

3 line(s) of initial conditions input follows.

Gas Pressure, $101330.0, \mathrm{~Pa}$ (absolute), 0.0,1/m,0.0,1/m,0.0,1/m,1,50,1,1,1,1,

Liquid Saturation, $0.5,0.0,1 / \mathrm{m}, 0.0,1 / \mathrm{m}, 0.0,1 / \mathrm{m}, 1,50,1,1,1,1$,

Temperature, 70.0 , Celsius, $0.0,1 / \mathrm{m}, 0.0,1 / \mathrm{m}, 0.0,1 / \mathrm{m}, 1,50,1,1,1,1$,

-Sources sinks

o line(s) of sources s sinks input follows.

- Iiquid Pathline Trace

o line(s) of liquid pathline trace input follows.

Output Control

$1,50,1,1,1,1$, xyplane,

0 line(s) of output control input follows.

$35,1,1,40,1,1,45,1,1,50,1,1,1$

0 line(s) of output control input follows.

7 line(s) of output control input follows.

Liquid Pressure.

Gas Pressure,

Temperature,

Liquid Saturation,

Gas Phase Vapor Mass Fraction,

X-Dir. Liquid Velocity,

X-Dir. Gas Velocity,

7 line(s) of output control input follows.

Iiquid Pressure,

Gas Pressure,

Temperature,

Liquid Saturation,

Gas Phase Vapor Mass Fraction,

X-Dir. Liquid Velocity,

x-Dir. Gas Velocity.

surface Flux Integrator

0 line(s) of surface flux integrator input follows.

Rock List

1 line(s) of rock list Input follows.

Porous Media, 


\subsection{Unsaturated Transport}

This problem considers transport in a one-dimensional, semi-infinite, horizontal tube of partially saturated soil. The object is to determine the concentration field in a tube of soil that has a uniform initial concentration and moisture content and in which the upstream boundary is subject to a prescribed moisture content and solute concentration. Transport processes include both advection and hydrodynamic dispersion, with both the hydraulic diffusivity and the hydraulic dispersion being functions of moisture content. This problem was proposed by Ross et al. (1982) as a test problem, and results are compared with those obtained from a semi-analytical solution reported by Smiles et al. (1977). The water transport problem on which this transport case is imposed is the horizontal infiltration problem for which Philip (1955) provided a semi-analytical solution.

\subsubsection{Problem Domain and Input Parameters}

This problem considers transport in an unsaturated flow field calculated for a one-dimensional, horizontal pipe. The objective is to determine the species concentration field in a tube of soil with porosity 0.45 , a uniform initial species concentration and moisture content, and an upstream boundary that is maintained at a prescribed moisture content and a prescribed solute concentration. Transport processes includes both advection and hydrodynamic dispersion, with both hydraulic diffusivity and hydraulic dispersion as functions of moisture content $\left(D_{S}(\theta)=\theta D\right.$, where $D$ is equal to $1.0 \mathrm{~cm} 2 /$ day in this problem).

The liquid-phase boundary conditions are saturation commencing at time zero on the west (left, or $x=0 \mathrm{~cm}$ ) boundary and a constant saturation of 0.444 (initial condition) at the east (right, or $x=20 \mathrm{~cm}$ ) boundary. The species boundary conditions are $1.0 \mathrm{~g} / \mathrm{L}$ concentration commencing at time zero at the west boundary and $0.1 \mathrm{~g} / \mathrm{L}$ at the east boundary. MSTS species concentrations are always indicated in terms of moles or curies per volume, but mass per volume ( $\mathrm{g} / \mathrm{L}$ in this case) may also be used so long as this usage is consistent. Note that concentrations at boundaries are expressed with respect to the volume of the phase (i.e., $1.0 \mathrm{~g} / \mathrm{L}$ of water at the west boundary) while concentrations for initial conditions are expressed with respect to total volume. The initial conditions are: gas pressure, 101,325 $\mathrm{Pa}$ (absolute); liquid saturation, 0.444; species concentration, $0.1 \mathrm{~g} / \mathrm{L}$; and temperature, $20.0^{\circ} \mathrm{C}$. The species concentration proposed by Ross et al. (1982) $(0.1$ $\mathrm{g} / \mathrm{L}$ ) is in terms of water volume, so we must express this value in terms of total volume for MSTS. Multiplying $0.1 \mathrm{~g} / \mathrm{L}$ by the moisture content (which is the ratio of water volume to total volume), we obtain $0.1 \mathrm{~g} / \mathrm{L}_{\text {wtr }} \times 0.2 \mathrm{~L}_{w t} / \mathrm{L}_{\text {tot }}=0.02 \mathrm{~g} / \mathrm{L}_{\text {rot }}$. 


\subsubsection{Semi-Analytic Solution}

Philip (1955) introduced a semi-analytical solution to the one-dimensional flow domain represented in this unsaturated transport problem. Smiles et al. (1977) reported the semi-analytical solution for the transport.

\subsubsection{Numerical Accuracy}

The spatial and temporal discretization requires careful attention so that stability is ensured in solving the dilute species mass conservation equation. A discretization or time stepping that is too large may result in excessive numerical dispersion, which would result in unacceptable error in predictions of solute transport. We base the maximum grid size for the domain in which dilute species transport is to be modeled on the criterion (Huyakorn and Pinder 1983; Price et al. 1966)

$$
P e=\frac{u \Delta x}{\tau_{\ell} s_{\ell} n_{D} D} \leq 2
$$

$$
\text { where } \begin{aligned}
P e & =\text { Peclet Number } \\
u & =\text { Darcy velocity in } x \text {-direction, } \mathrm{cm} / \text { day } \\
\Delta x & =\text { grid cell length in } x \text {-direction, } \mathrm{cm} \\
\tau_{\ell} & =\text { liquid-phase tortuosity } \\
s_{\ell} & =\text { liquid saturation } \\
n_{D} & =\text { diffusive porosity } \\
D & =\text { molecular diffusivity, } \mathrm{cm}^{2} / \text { day. }
\end{aligned}
$$

The expression in Equation (8.13) reflects the one-dimensional nature of this problem (i.e., we are not concerned with the Peclet Number in other dimensions). The liquid-phase tortuosity in this problem is 1 , the diffusive porosity is 0.45 , and the molecular diffusivity is $1.0 \mathrm{~cm}^{2} /$ day. To determine the maximum permissible $\Delta x$ requires values for the Darcy velocity in the $x$-direction and the liquid saturation, both of which are time-varying in this problem. By performing preliminary simulations with MSTS for the water conservation equation only, we find that early peak velocities are on the order of $22.3 \mathrm{~cm} /$ day, and the associated volumetric moisture content (equal to the product $\left.s_{\ell} n_{D}\right)$ is 0.445 . Substituting these values into Equation (8.13), we obtain 


$$
\Delta x \leq \frac{2 \tau_{\ell} s, n_{D} D}{u}=\frac{2(1.0)(0.445)\left(1.0 \mathrm{~cm}^{2} / \text { day }\right)}{22.3 \mathrm{~cm} / \text { day }}=0.0399 \mathrm{~cm} \approx 0.04 \mathrm{~cm}
$$

The maximum permissible time step is similarly determined by following the criterion

$$
\operatorname{Cr}=\frac{u \Delta t}{\Delta x} \leq 1
$$

where $\mathrm{Cr}$ is the Courant Number. This is merely a mathematical expression for the criterion that the time required for fluid to transit the grid cell length must be less than the time-step duration. Again, substituting:

$$
\Delta t \leq \frac{\Delta x}{u}=\frac{0.04 \mathrm{~cm}}{22.3 \mathrm{~cm} / \mathrm{day}}=0.00179 \text { day } \approx 150 \text { seconds }
$$

Therefore, the input file shown in Exhibit 8.10 uses a grid cell length of $0.04 \mathrm{~cm}$ for the portion of the domain between 0.0 and $4.5 \mathrm{~cm}$ (Exhibit 8.10, Grid Geometry card, lines 31-190). Somewhat larger grid cells are used beyond $4.5 \mathrm{~cm}$ because that is the maximum distance we expect the solute front to reach in the 0.11 day; hence, the $0.04-\mathrm{cm}$ resolution is not required beyond $4.5 \mathrm{~cm}$. The maximum time step is restricted to 150 seconds (Exhibit 8.10, Solution Schemes card, line 23). A final caution: nothing in the MSTS code will prevent the user from exceeding the Peclet or Courant limits discussed here. It is the responsibility of the modeler to ensure numerical accuracy through appropriate spatial- and temporal-discretization choices.

\subsubsection{Simulation Results and Comparison to Semi-Analytic Solution}

The total time simulated for this problem was 9504 seconds ( 0.11 day). The "dimensionless concentrations" at 0.01, 0.06, and 0.11 day reported in Ross et al. (1982) for the Smiles et al. (1977) semi-analytical solution are shown as solid lines in Figure 8.15. The MSTS solution for the input file shown in Exhibit 8.10 is shown as one dashed line (labeled " $\Delta x=0.04 \mathrm{~cm}$ " in the legend), while a second MSTS solution using a grid spacing of only $0.02 \mathrm{~cm}$ and a maximum time step of 75 seconds is shown as a second dashed line (" $\Delta \mathrm{x}=0.02 \mathrm{~cm}$ " in the legend). Note that decreasing the grid spacing (and hence the time spacing) to meet the Courant limit reduces the numerical dispersion for this strongly advection-driven problem. Further reductions in the time and space stepping would bring even better predictions compared to the semi-analytical solution, but at the price of greater computer time requirements to solve the problem. 


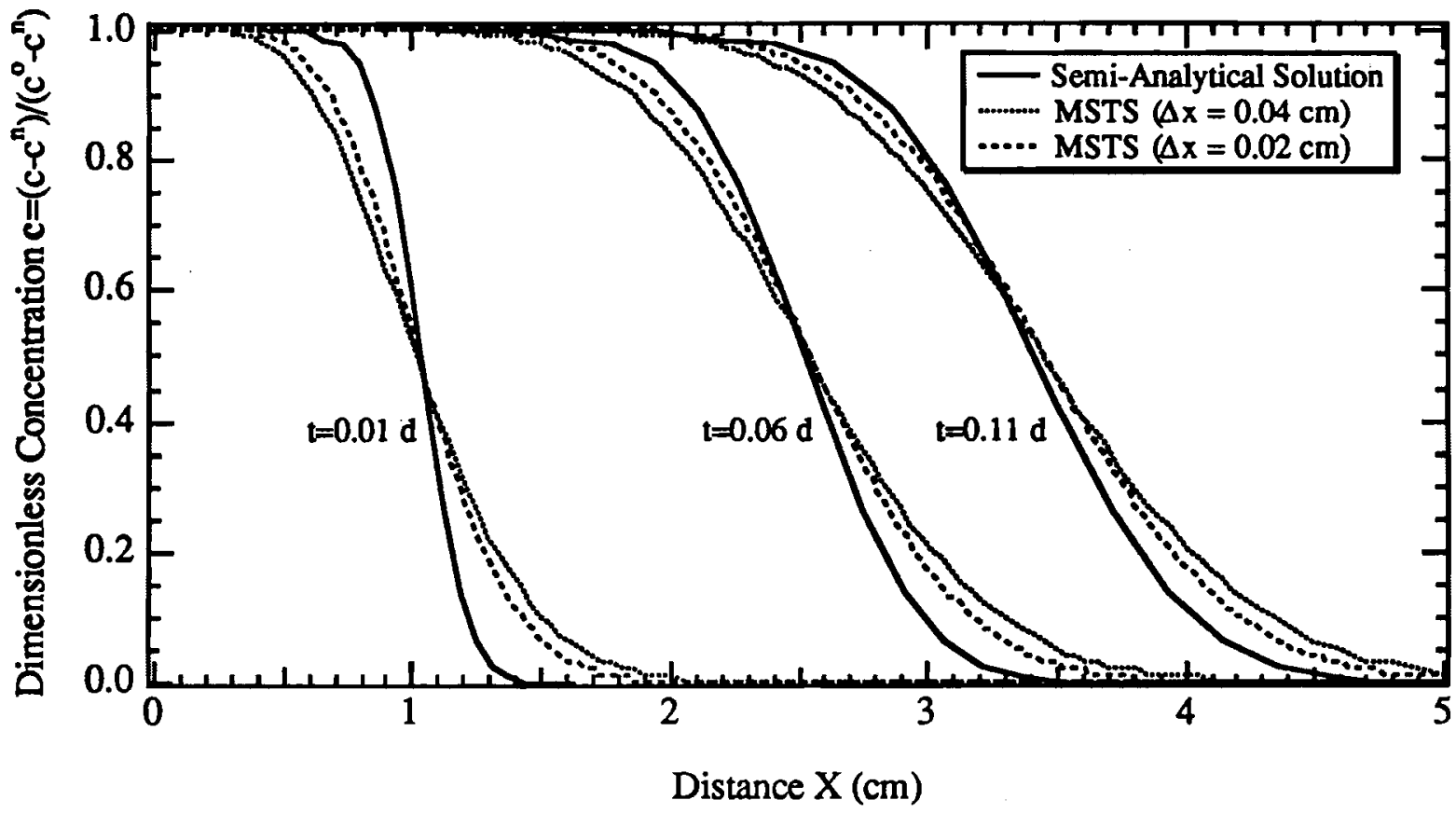

Figure 8.15. Dimensionless Solute Concentration Versus Distance at Various Times Using the Semi-Analytical Solution of Smiles et al. (1977) and MSTS Solutions With Two Spatial and Temporal Discretizations

The dimensionless concentration was not a direct output of MSTS, but was computed using MSTS output. MSTS reports species concentration in units of mol/L, where the volumetric measure (liters) refers to total volume (not water volume, as Smiles et al. [1977] reports it). Therefore, we divide each concentration reported by MSTS by the corresponding moisture content for the time and cell to obtain the concentration with respect to the aqueous phase. This value is converted into a dimensionless concentration by scaling it between the values at each boundary so that all concentrations have a value between 0 (initial condition) and 1 (maximum concentration). 
Exhibit 8.10. MSTS Input File for Unsaturated Transport Problem

\section{Line No. Input File}

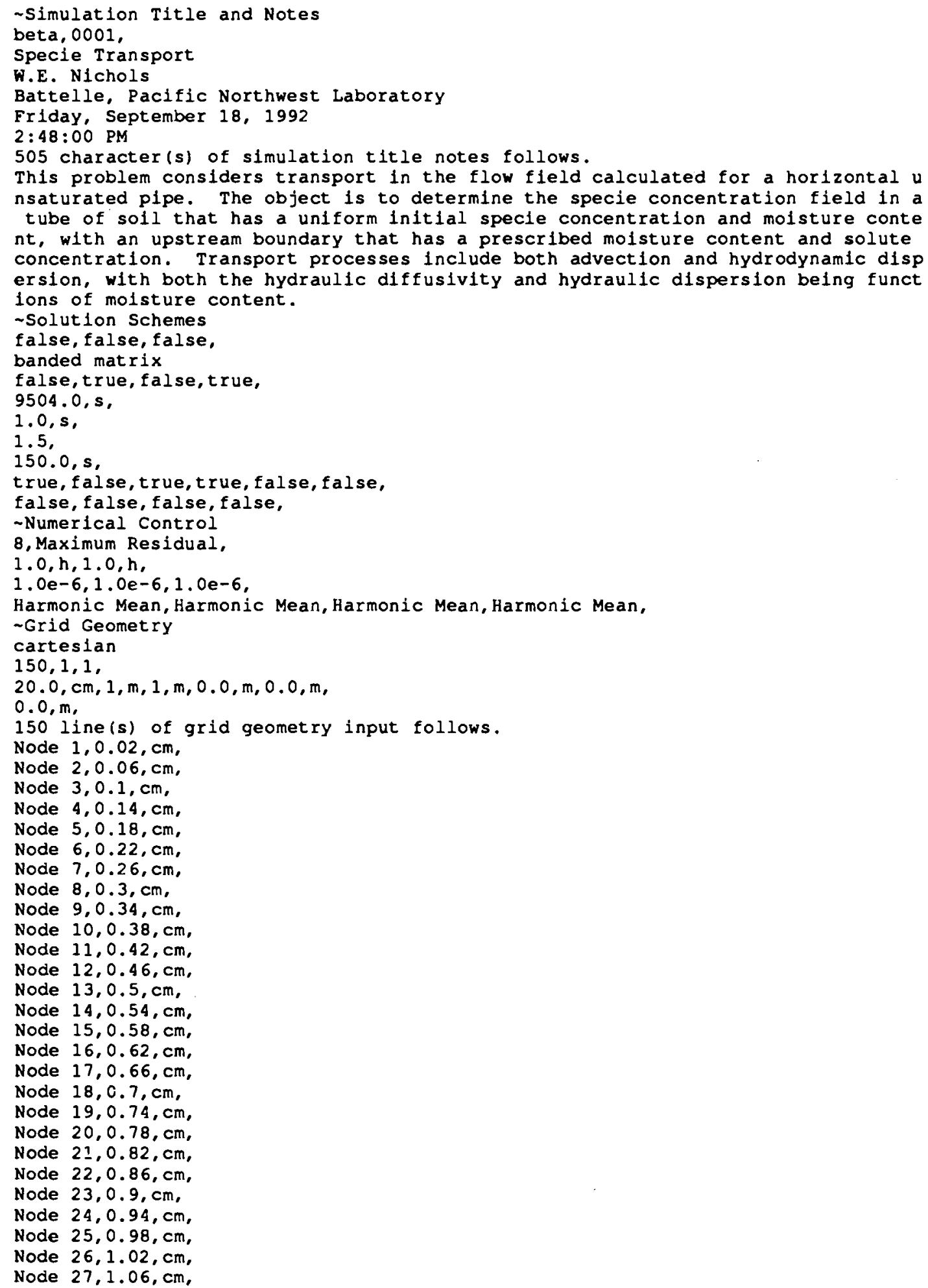


Exhibit 8.10. (Contd)

\section{Line No. Input File}

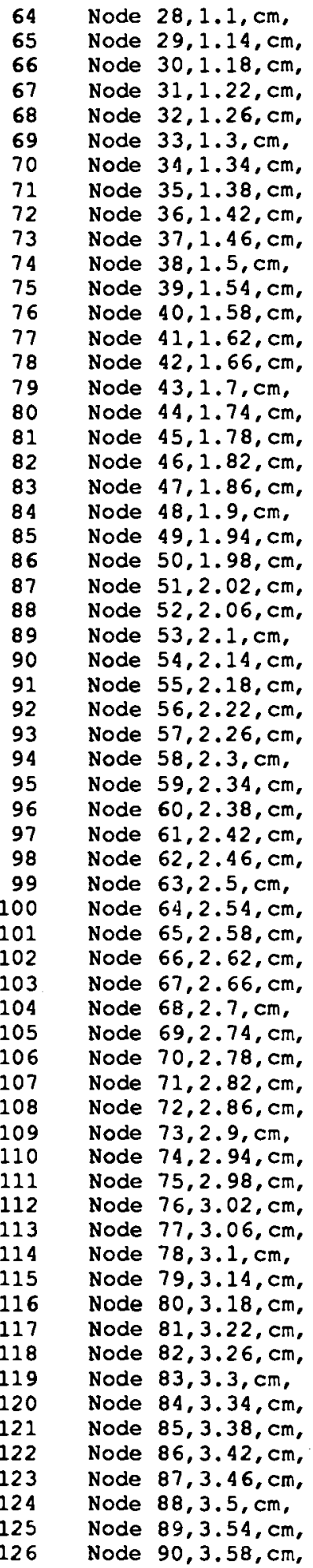


Exhibit 8.10. (Contd)

Line No Input File

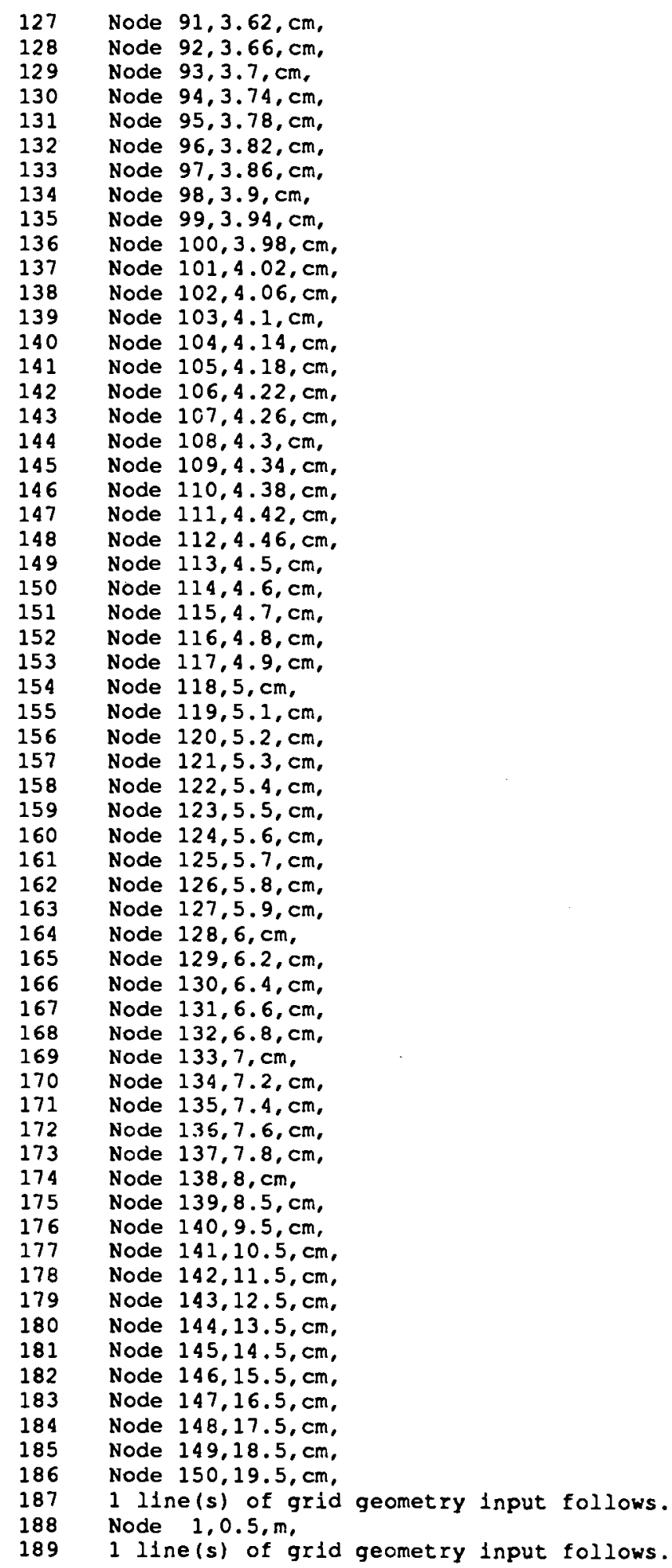




\section{Exhibit 8.10. (Contd)}

\section{Line No Input File}

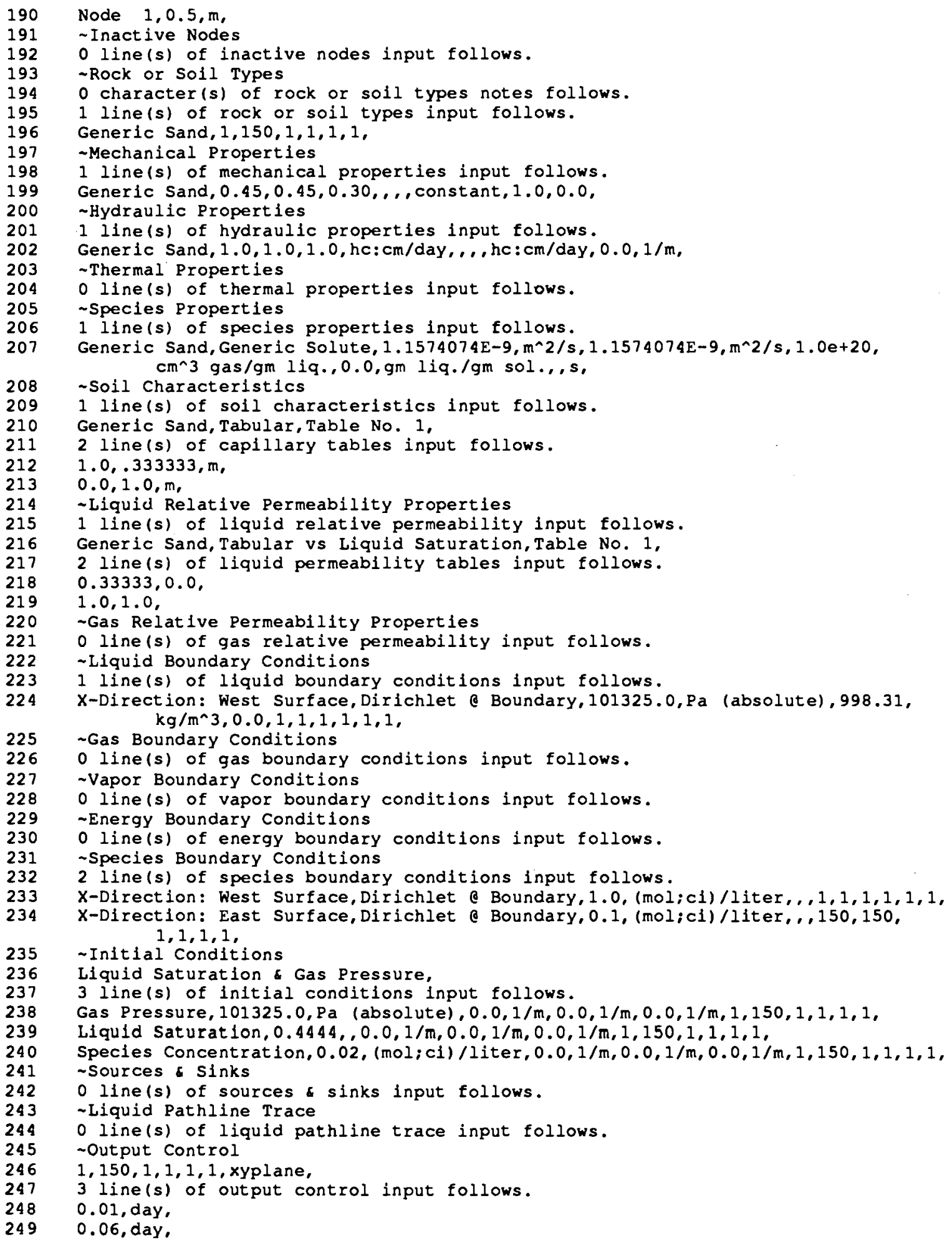




\section{Exhibit 8.10. (Contd)}

\section{Line No. Input File}

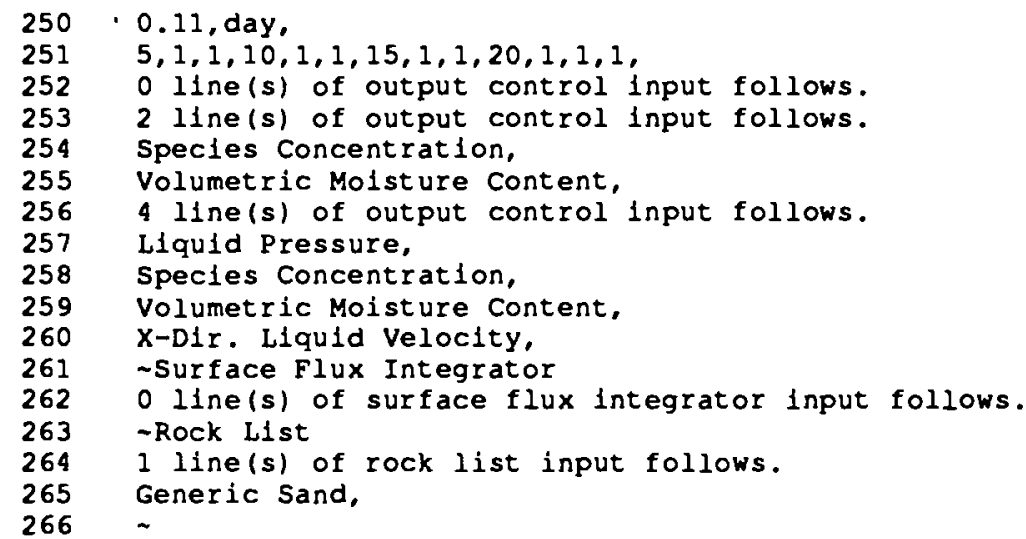




\subsection{References}

Baca, R. G., and S. O. Magnuson. 1990. Independent Verification and Benchmark Testing of the UNSAT-H Computer Code, Version 2.0. EGG-BEG-88112, Idaho National Engineering Laboratory, Idaho Falls, Idaho.

Burdine, N. T. 1953. "Relative Permeability Calculations from Pore-Size Distribution Data." Petroleum Trans., American Institute of Mining and Metallurgical Engineering, 198:71-77.

El-Kadi, A. I. 1987. INFIL. International Groundwater Modeling Center, Holcomb Research Institute, Indiana.

Fatt, I., and W. A. Klikoff. 1959. "Effect of Fractional Wettability on Multiphase Flow Through Porous Media." AIME Transactions 216:246.

Haverkamp, R., M. Vauclin, J. Touma, P. Wierenga, and G. Vachaud. 1977. "A Comparison of Numerical Simulation Models for One-Dimensional Infiltration." Soil Sciences America Journal 41:285-294.

Huyakorn, P. S., and G. F. Pinder. 1983. Computational Methods in Subsurface Flow. Academic Press, San Diego, California.

Leverett, M. C. 1941. "Capillary Behavior in Porous Solids." AIME Transactions 142:152.

Millington, R. J., and J. P. Quirk. 1959. "Permeability of Porous Media." Nature 183:387-388.

Moore, R. E. 1939. "Water Conduction from Shallow Water Tables." Hilgardia 12:383-426.

Mualem, Y. 1976. "A New Model for Predicting the Hydraulic Conductivity of Unsaturated Porous Media." Water Resources Research 12(3):513-522.

Philip, J. R. 1955. "Numerical Solution of Equations of the Diffusion Type with Diffusivity Concentration Dependent." Trans. Faraday Soc. 51:885-892.

Philip, J. R. 1957. "Numerical Solution of Equations of the Diffusion Type with Diffusivity Concentration-Dependent II." Australian Journal of Physics 10(2):29-42.

Price, H. S., R. S. Varga, and J. E. Warren. 1966. "Application of Oscillation Matrices to Diffusion-Convection Equations." J. Mathematical Physics 45:301-311.

Pruess, K. 1987. TOUGH User's Guide. LBL-20700, Lawrence Berkeley Laboratory, Berkeley, California.

Ross, B., J. W. Mercer, S. D. Thomas, and B. H. Lester. 1982. Benchmark Problems for Repository Siting Models. NUREG/CR-3097, U.S. Nuclear Regulatory Commission, Washington, D.C.

Smiles, D. E., J. R. Philip, J. H. Knight, and D. E. Elrick. 1977. "Hydrodynamic Dispersion During Absorption of Water by Soil." Soil Sciences America Journal 42:229-234. 
Touma, J., and M. Vauclin. 1986. "Experimental and Numerical Analysis of Two-Phase Infiltration in a Partially Saturated Soil." Transport in Porous Media (1986):27-55.

Udell, K. S., and J. S. Fitch. 1985. "Heat and Mass Transfer in Capillary Porous Media Considering Evaporation, Condensation, and Non-Condensible Gas Effects." Presented at the 23rd ASME/AIChE National Heat Transfer Conference, Denver, August 1985.

van Genuchten, M. T. 1980. "A Closed Form Equation for Predicting the Hydraulic Conductivity of Unsaturated Soils." Soil Sciences America Journal 44:982-998.

van Wylen, G. J., and R. E. Sonntag. 1985. Fundamentals of Classical Thermodynamics. Wiley, New York.

White, M. D., and W. E. Nichols. 1992. MSTS: Multiphase Subsurface Transport Simulator Theory Manual. PNL-8636, Pacific Northwest Laboratory, Richland, Washington. 


\section{Index}

Air entrapment effects $\ldots \ldots \ldots \ldots \ldots \ldots 2.4 \quad$ CPU execution time limit

Air mass conservation equation ..... 2.6-2.7 Darcy's law $2.4,2.6-2.7$

Anisotropic $\ldots \ldots \ldots \ldots \ldots \ldots \ldots . .2 .1$

Datum

Application scope, MSTS .......... 2.4

Debug file

Banded matrix solver $\ldots \ldots \ldots \ldots .2 .2,6.8$

parameterization ...........4.3

Density $\ldots \ldots \ldots \ldots \ldots \ldots \ldots .6 .27$

Dimensionality ............... 2.1

Binary diffusion $\ldots \ldots \ldots \ldots \ldots \ldots .6$

Boundary conditions $\ldots \ldots \ldots \ldots .2 .2,6.43$ inactive nodes $\ldots \ldots \ldots \ldots \ldots 6.18$ parameter $\ldots \ldots \ldots \ldots \ldots \ldots .4 .3$

Brooks and Corey relations $\ldots \ldots \ldots \ldots 7.2$ liquid relative permeability $\quad 6.35-6.36$ moisture retention .........6. 6.31

Burdine function

Computational effort 2.10

Conductivity

hydraulic $\ldots \ldots \ldots \ldots .65 .9,6.22,6.25$

thermal $\ldots \ldots \ldots \ldots \ldots .6 .22,6.27$

units $\ldots \ldots \ldots \ldots \ldots \ldots \ldots .6 .9$

Conjugate gradient solver $\ldots \ldots \ldots \ldots \quad 2.2,6.8$ parameterization $\ldots \ldots \ldots \ldots \ldots .4 .3$

Conservation equations

air mass $\ldots \ldots \ldots \ldots \ldots \ldots . \quad 2.6-2.7$

species mass $\ldots \ldots \ldots \ldots \ldots \ldots \ldots .2 .9$

thermal energy $\ldots \ldots \ldots \ldots \ldots, \quad 2.7-2.8$

water mass $\ldots \ldots \ldots \ldots \ldots . \quad 2.5-2.6$

Dirichlet boundary $\ldots \ldots \ldots \ldots \ldots \ldots .2 .2$

Dual-porosity model $\quad \ldots \quad 6.23,6.35-6.36,7.5$ moisture retention $\ldots \ldots \ldots \ldots 6.32$

Energy Boundary Conditions card .... 6.52

Equation coupling $\ldots \ldots \ldots \ldots \ldots \ldots 2.2$

Fatt and Klikoff relations ........... 7.4

gas relative permeability ...... 6.40

liquid relative permeability $\ldots .66 .36$

File management $\ldots \ldots \ldots \ldots \ldots \ldots .4 .1$

Fixed properties $\ldots \ldots \ldots \ldots \ldots \ldots .6 .8$

Fractures, discrete $\ldots \ldots \ldots \ldots \ldots \ldots .2 .4$

Gas Boundary Conditions card ...... 6.47

Gas Relative Permeability card ...... 6.40

Governing equations $\ldots \ldots \ldots \ldots 2.2,2.5$

Grid Geometry card ........... 6.14

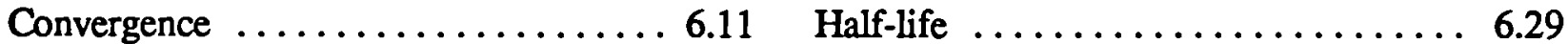

Coordinate systems $\ldots \ldots \ldots \ldots \ldots \ldots .2 .1 \quad$ Havercamp relations $\ldots \ldots \ldots \ldots \ldots .7 .3$

Corey relations $\ldots \ldots \ldots \ldots \ldots \ldots . . .7 .3$

gas relative permeability .......6.40

liquid relative permeability $\ldots .6 .36$

Courant Number ............. 8.64 liquid relative permeability ..... 6.36 moisture retention $\ldots \ldots \ldots \ldots 6.32$

Heat transfer

diffusive $\ldots \ldots \ldots \ldots \ldots \ldots .2 .4$

latent $\ldots \ldots \ldots \ldots \ldots \ldots \ldots, 2.4$

sensible $\ldots \ldots \ldots \ldots \ldots \ldots \ldots . .4 .4$

Index.1 


\section{Index}

Heterogeneity $\ldots \ldots \ldots \ldots \ldots \ldots \ldots .2 .1$

Hydraulic Properties card ...... 5.9, 6.25 Hysteretic effects $\ldots \ldots \ldots \ldots \ldots \ldots .4 .4$

Inactive nodes $\ldots \ldots \ldots \ldots 2.2,4.3,6.2,6.18$

Inactive Nodes card $\ldots \ldots \ldots \ldots \ldots \ldots .6 .18$

Initial Conditions card $\ldots \ldots \ldots \ldots .5 .12,6.58$

Initial Conditions Only option $\ldots \ldots \quad 5.5,6.8$

Input file $\ldots \ldots \ldots \ldots \ldots \ldots .4 .2,5.13$

Kinetic modeling ............ 2.4-2.5

Leverett relations ................. 7.4

moisture retention $\ldots \ldots \ldots \ldots .6 .32$

Liquid Boundary Conditions card . 5.11, 6.44

Liquid Pathline Trace card $\ldots \ldots \ldots .6 .70$

Liquid Relative Permeability card 5.10, 6.35

Machine specification $\ldots \ldots \ldots \ldots 4.4,5.13$

Mechanical dispersion $\ldots \ldots \ldots \ldots \ldots . .2 .8$

Mechanical Properties card ...... 5.9, 6.23

Millington and Quirk $\ldots \ldots \ldots \ldots \ldots .6 .23$

Molecular diffusion coefficient ...... 6.29

Mualem function $\ldots \ldots \ldots \ldots \ldots \ldots \ldots .6 .35$

Neumann boundary $\ldots \ldots \ldots \ldots \ldots .2 .2$

Newton-Raphson iteration $\quad \ldots \quad 1.1,2.2,6.11$

Numerical Control card ......... 5.7,6.11

Output control $\ldots \ldots \ldots \ldots \ldots \ldots \ldots .2 .2$

Output Control card

$5.13,6.72$

Output file
Output time

Parameterization

$4.2,5.13$

Partition coefficient $\ldots . \ldots \ldots \ldots . \ldots 6.29$

Peclet Number $\ldots \ldots \ldots \ldots \ldots \ldots .6 .63$

Permeability .................. 6.25

gas relative $\ldots \ldots \ldots \ldots \ldots, 6.2,6.40$

intrinsic $\ldots \ldots \ldots \ldots \ldots \ldots, 5.9$

liquid relative $\ldots \ldots \ldots \ldots 6.6 .2,6.35$

Plot file

6.73

Porosity $\ldots \ldots \ldots \ldots \ldots \ldots, 2.3,5.9,6.22$

diffusive $\ldots \ldots \ldots \ldots \ldots \ldots 2.3,6.23$

dual-porosity model $\ldots \ldots \ldots \quad 2.4,6.36$

effective $\ldots \ldots \ldots \ldots \ldots \ldots, 2.3,6.23$

fracture $\ldots \ldots \ldots \ldots \ldots \ldots \ldots, 6.23$

total $\ldots \ldots \ldots \ldots \ldots \ldots, 2.3,6.23$

Radioactive decay ............. 6.29

Reference node $\ldots \ldots \ldots \ldots \ldots \ldots .6 .72$

Representative elementary volume $\ldots \ldots 2.3$

Restart file ................. 4.2

Restart from Time Zero option ....... 6.8

Restart option $\ldots \ldots \ldots \ldots \ldots \ldots .2 .2,6.8$

Revision number $\ldots \ldots \ldots \ldots \ldots$ 6.5-6.6

Rock or Soil Types card $\ldots \ldots \ldots .5 .8,6.20$

Sample problems $\ldots . \ldots \ldots \ldots \ldots .8 .1$

Saturated boundary $\ldots \ldots \ldots \ldots \ldots .2 .2$

Screen echo $\ldots \ldots \ldots \ldots \ldots \ldots \ldots, 6.8$

Simulation Title card $\ldots \ldots \ldots \ldots \ldots \ldots, 6.6$

Simulation, running $\ldots \ldots \ldots \ldots \ldots . .16$

Soil Characteristics card ....... 5.10,6.31

Index.2 


\section{Index}

Solution modes or options $\ldots \ldots \ldots \quad$ 2.9-2.11

Air ................... 2.13

Air-Energy $\ldots \ldots \ldots \ldots \ldots \ldots .2 .17$

Energy $\ldots \ldots \ldots \ldots \ldots \ldots \ldots .2 .15$

Water $\ldots \ldots \ldots \ldots \ldots \ldots \ldots .2 .12$

Water-Air ................ 2.14

Water-Air-Energy ........... 2.18

Water-Energy $\ldots \ldots \ldots \ldots \ldots \ldots 2.16$

Solution Schemes card $\ldots \ldots \ldots \ldots \quad 5.4,6.8$

Sources and sinks $\ldots \ldots \ldots \ldots \ldots \ldots 2.3$

Unit-gradient boundary $\ldots \ldots \ldots \ldots \ldots .2 .2$

Units, Système International $\ldots \ldots \ldots .2 .4$

van Genuchten relations ........ 5.10,7.1

in dual-porosity model ....... 7.5

liquid relative permeability $\ldots \ldots 6.35$

moisture retention $\ldots \ldots \ldots \ldots 6.31$

Vapor Boundary Conditions card $\ldots . .6 .50$

Vapor pressure lowering $\ldots \ldots \ldots \ldots 6.8$

Sources and Sinks card .......... 6.61

Variable saturation $\ldots \ldots \ldots \ldots \ldots \ldots .2 .3$

Space dependence $\ldots \ldots \ldots \ldots \ldots \ldots .2 .1$

Version name $\ldots . \ldots \ldots \ldots \ldots .6 .6 .5-6.6$

Species Boundary Conditions card $\ldots .6 .55$ Wallclock time limit $\ldots \ldots \ldots \ldots .7,6.11$

Species mass conservation equation $\ldots . .2 .9$ Water mass conservation equation $\ldots$ 2.5-2.6

Species Properties card .......... 6.29

Specific heat $\ldots \ldots \ldots \ldots \ldots \ldots .6 .27$

Steady-state solutions $\ldots \ldots \ldots \ldots \ldots . .1$

Surface file $\ldots \ldots \ldots \ldots \ldots \ldots \ldots . .2$

Surface Flux Integrator card $\ldots \ldots \ldots 6.76$

Système International (SI) $\ldots \ldots \ldots \ldots .4$

Thermal energy conservation equation $\quad 2.7-2.8$

Thermal Properties card $\ldots \ldots \ldots \ldots .6 .27$

Time dependence $\ldots \ldots \ldots \ldots \ldots \ldots . . .1$

Time step

acceleration factor $\ldots \ldots \ldots \ldots, 6.8$

initial .................. 6.8

maximum $\ldots \ldots \ldots \ldots \ldots \ldots 6.8$

Tortuosity .............. $6.22-6.23$

Touma and Vauclin relations .......... 7.5

gas relative permeability . . . . 6.40

liquid relative permeability $\ldots .66 .36$ 


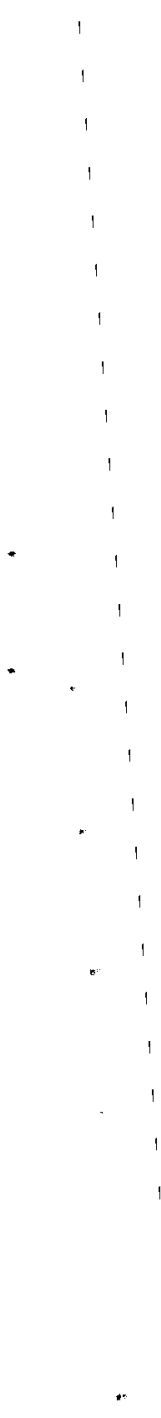




\section{DISTRIBUTION}

No. of

Copies

\section{QFFSITE}

12 DOE Office of Scientific and Technical Information

Technical Information Center

P.O. Box 62

Oak Ridge, TN 37830

8 Yucca Mountain Site Characterization Project Office

U.S. Department of Energy

P.O. Box 98608

Las Vegas, NV 89193-8608

ATTN: J. M. Boak

J. R. Dyer

C. P. Gertz (5)

J. J. Lorenz

15 DOE Nevada Field Office

U.S. Department of Energy

P.O. Box 98518

Las Vegas, NV 89193-8518

ATTN: D. R. Elle

P. K. Fitzsimmons

Tech Information Officer (12)

C. L. West

Science and Technology Division

Office of Scientific \& Technical

Information

U.S. Department of Energy

P.O. Box 62

Oak Ridge, TN 37831

ATTN: A. T. Tamura
No. of

Copies

8 U.S. Department of Energy

1000 Independence Avenue, S.W.

Washington, DC 20585

ATTN: S. J. Brocoum

RW-22

P. Bunton

RW-331

L. Desell

RW-331

R. A. Milner

RW-332

G. J. Parker

J. Roberts

RW-322

S. Rousso

RW-30

T. Wood

RW-50

RW-52

C. Anderson

National Academy of Sciences

2101 Constitution Ave. NW

Harris Bldg, Rm 456

Washington, DC 20418

2 Agency for Nuclear Projects

State of Nevada

Evergreen Center, Suite 252

1802 N. Carson St.

Carson City, NV 89710

ATTN: C. H. Johnson

R. R. Loux

C. R. Allen

Nuclear Waste Technical Review Board 1000 E. California Boulevard

Pasadena, CA 91106

E. Anderson

Mountain West Research

Southwest, Inc.

2901 N. Central Ave., \#1000

Phoenix, AZ 85012-2730

Argonne National Laboratory

9700 S. Cass Avenue

Argonne, IL 60439

ATTN: A. Anderson

Bldg 362

Distr.1 
No. of

Copies

M. L. Baughman

35 Clark Road

Fiskdale, MA 01518

C. G. Bell, Jr.

Professor of Civil Engineering

Civil and Mechanical Engineering

University of Nevada, Las Vegas

4505 S. Maryland Parkway

Las Vegas, NV 89154

\section{J. A. Blink}

Deputy Project Leader

Lawrence Livermore Natl Laboratory

101 Convention Center Drive

Suite 280, MS 527

Las Vegas, NV 89109

S. Bradhurst

P.O. Box 1510

Reno, NV 89505

L. Bradshaw

Nye County District Attorney

P.O. Box 593

Tonopah, NV 89049

G. D. Brewer

Nuclear Waste Technical Review Board

The Dana Building, Room 3516

University of Michigan

Ann Arbor, MI 48109-1115

R. J. Budnitz

President, Future Resource Associates

2000 Center Street, Suite 418

Berkeley, CA 94704

R. L. Bullock

Raytheon Services Nevada

Suite P-250 (MS-403)

101 Convention Center Dr.

Las Vegas, NV 89109
No. of

Copies

J. E. Cantlon, Chairman

Nuclear Waste Technical Review Board

1795 Bramble Drive

East Lansing, MI 48823

2 City of Caliente

P.O. Box 158

Caliente, NV 89008

ATTN: J. Foremaster

G. Van Roekel

City of Boulder City

Community Development \& Planning

P.O. Box 61350

Boulder City, NV 89006

City of North Las Vegas

Community Planning \& Development

P.O. Box 4086

North Las Vegas, NV 89030

City of Las Vegas

Economic Development Department

400 E. Stewart Avenue

Las Vegas, NV 89101

Clark County

Department of Comprehensive Planning

225 Bridger Ave., 7th Floor

Las Vegas, NV 89155

B. W. Colston

Las Vegas Branch

Raytheon Services Nevada

P.O. Box 95487, MS 416

Las Vegas, NV 89193-5487

T. A. Cotton

JK Research Associates, Inc.

4429 Butterworth Place, N.W.

Washington, DC 20016 
No. of

Copies

R. H. Cuenca

Oregon State University

Department of Bioresource Engineering

Gilmore Hall 106

Corvallis, OR 97331

2 Desert Research Institute

P.O. Box 60220

Reno, NV 89506

ATTN: J. Fordham

D. Rhode

P. A. Domenico

Nuclear Waste Technical Review Board

Geology Department

Texas A \& M University

College Station, TX 77843

C. E. Ezra

YMP Support Office Manager

EG7G Energy Measurements, Inc.

P.O. Box 1912, MS V-02

Las Vegas, NV 89125

Exploratory Shaft Test Manager

Los Alamos National Laboratory

101 Convention Center Drive

Suite 820, Mail Stop 527

Las Vegas, NV 89101

L. J. Fiorenzi

P.O. Box 257

Eureka, NV 89316

L. D. Foust

Nevada Site Manager

TRW Environmental Safety Systems

101 Convention Center Dr.

Suite 540, MS 423

Las Vegas, NV 89109

B. Hastings

TRW Environmental Safety Systems

2650 Park Tower Drive, Suite 800

Vienna, VA 22180
No. of

Copies

T. Hay, Executive Assistant

Office of the Governor

State of Nevada

Capitol Complex

Carson City, NV 89710

J. Hayes

P.O. Box 490

Goldfield, NV 89013

D. Hedges

Roy F. Weston, Inc.

4425 Spring Mountain Road, Suite 300

Las Vegas, NV 89102

D. Hillel

Department of Plant and Soil Science

12A Stockbridge Hall

University of Massachusetts

Amherst, MA 01003

R. G. Hills

Department of Mechanical Engineering

Box 30001, Department 3450

New Mexico State University

Las Cruces, NM 88003

R. Horton

Department of Agronomy

Iowa State University

Ames, IA 50011

5 INTERA, Inc.

101 Convention Center Drive

Phase II, Suite 540

Las Vegas, NV 89193

ATTN: R. W. Nelson (2)

S. Pawah

A. E. Van Luik

H. N. Kalia

G. G. Katul

Department of Hydrologic Science

Veihmeyer Hall

University of California

Davis, CA 95616

Distr.3 
No. of

Copies

D. Langmuir

Nuclear Waste Technical Review Board 109 S. Lookout Mountain Creek

Golden, CO 80401

7 Lawrence Berkley Laboratory

Berkeley, CA 94720

ATTN: E. P. Binnall

W. W.-L. Lee

T. N. Narasimhan

W. J. O'Connell

K. Pruess

C. F. Tsang

J. Wang

5 Lawrence Livermore National Laboratory

P.O. Box 808

Livermore, CA 94551

ATTN: T. A. Buscheck

D. Chesnut

W. L. Clarke

W. Halsey

W. J. O'Connell

Lincoln County Commission

Lincoln County

P.O. Box 90

Pioche, NV 89043

H. Loo

Weston House Idaho Nuclear Co.

P.O. Box 4000, M/S 5219

Idaho Falls, ID 83415

2 Los Alamos National Laboratory

P.O. Box 1663

Los Alamos, NM 87545

ATTN: J. A. Canepa

R. Rundberg
No. of

Copies
A. Makhijani

Institute for Energy and Environmental

Research

6935 Laurel Avenue

Takoma Park, MD 20912

C. John Mann

Department of Geology

245 Natural History Bldg.

1301 West Green Street

Urbana, Il 61801

M. Marshall

c/o LATIR Energy Consultants

Route 7, Box 126 B

Old Santa Fe Trail

Santa Fe, NM 87505

J. E. Martin

Asst Professor of Radiological Health

University of Michigan

School of Public Health

Ann Arbor, MI 48109

J. Massmann

Department of Civil Engineering

306 More Hall, FX-10

University of Washington

Seattle, WA 98195

J. J. McKetta, Jr.

Nuclear Waste Technical Review Board

Department of Chemical Engineering

CPE Building 1.450

Austin, TX 78712

H. R. Meyer

C.N.S.I.

750 East Park Drive, Suite 200

Harrisburg, PA 17111

I. Miller

Golder Associates

4104 184th Avenue N.E.

Redmond, WA 98052 
No. of

Copies

R. H. Neill

Director, New Mexico Environmental Evaluation Group

7007 Wyoming Boulevard, N.E.

Suite F-2

Albuquerque, NM 87109

D. W. North

Nuclear Waste Technical Review Board

Decision Focus, Inc.

4984 El Camino Real

Los Altos, CA 94062

2 North Carolina Agricultural and Technical University

Department of Civil Engineering

Greensboro, NC 27411

ATTN: E. U. Nzewi

L. E. Peele

NRC Document Control Desk

Divison of Waste Management

Nuclear Regulatory Commission

Washington, DC 20555

Nye County

Planning Department

P.O. Box 153

Tonopah, NV 89049

2 Oak Ridge National Laboratory

P.O. Box 2008

Oak Ridge, TN 37831

ATTN: A. G. Croff

F. O. Hoffman

MS-6235

ONWI Library

Battelle Columbus Laboratory

Office of Nuclear Waste Isolation

505 King Ave.

Columbus, $\mathrm{OH} 43201$
No. of

Copies

T. Pigford

Department of Nuclear Engineering

4153 Etcheverry Hall

University of California

Berkeley, CA 94720

J. Pitts

Lincoln County Courthouse

Pioche, NV 89043

V. Poe

P.O. Box 1026

Hawthorne, NV 89415

P. T. Prestholt

NRC Site Representative

301 E. Stewart Avenue, Room 203

Las Vegas, NV 89101

D. L. Price

Nuclear Waste Technical Review Board

1011 Evergreen Way

Blacksburg, VA 24060

M. Reeves

INTERA, Inc.

6850 Austin Crr. Blvd, Suite 300

Austin, TX 78731

3 Reynolds Electrical \& Engineering Co.

P.O. Box 98521

Las Vegas, NV 89193-8521

ATTN: M. J. Dorsey MS-407

D. L. Fraser MS-555

R. F. Pritchett MS-408

J. Riley

Heinman Research Laboratories

Charlotte, NC 28207

B. Ross

Disposal Safety, Inc.

1660 L. Street, N.W., Suite 314

Washington, DC 20036

Distr.5 
No. of

Copies

G. Ross

Heath, Chairman

College of Ocean \& Fishery Sciences

583 Henderson hall

University of Washington

Seattle, WA 98185

5 Roy F. Weston, Inc.

955 L'Enfant Plaza, S.W.

Washington, D.C. 20024

ATTN: L. Rickertsen

E. L. Snow

Chris Charles

Janet Docka

Technical Info. Center

8 Sandia National Laboratory

Albuquerque, NM 87185

ATTN: R. W. Barnard

6312

G. E. Barr

6312

F. W. Bingham 6312

T.E. Blejwas $\quad 6302$

R. R. Eaton 1513

P. G. Kaplan 6312

M. J. Martinez 6312

M. A. Wilson 6312

F. W. Schwartz

Dept of Geololgy and Mineralogy

The Ohio State University

1090 Carmack Road

Columbus, OH 43210-1398

3 Science Applications International Corporation (SAIC)

101 Convention Center Blvd, Suite 450

Las Vegas, NV 89109

ATTN: A. Brandstetter

P. L. Cloke

M. D. Voegele

B. Selinder

190 W. First St.

Fallon, NV 89406
No. of

Copies

L. Shepard

Ogden Environmental \& Energy

Services

7301A Indian School Rd., NE

Albuquerque, NM 87110

4 Southwest Research Institute

6220 Culebra Road

San Antonio, TX 78228

ATTN: B. Sagar (3)

J. Walton

C. Thistlethwaite, AICP

Associate Planner

Inyo County Planning Dept.

Drawer L

Independence, CA 93526

2 University of California at Riverside

Department of Soils

Riverside, CA 92502

ATTN: W. A. Jury

M. Th. van Genuchten

U.S. Bureau of Reclamation

Code D-3790

P.O. Box 25007

Denver, CO 80225

3 U.S. Environmental Protection Agency 401 "M" Street, S.W.

Washington, DC 20460

ATTN: D. M. Clark

A-101F

K. W. Conway

W. Russo

A-101F

ANR 460

2 U.S. Geological Survey

2255 N. Gemini Drive

Flagstaff AZ 86001

ATTN: J. H. Sass

S. C. Wu

U.S. Geological Survey

345 Middlefield Road

Menlo Park, CA 94025

ATTN: E. J. Helley 
No. of

Copies

6 U.S. Geological Survey

P.O. Box 25046

Denver CO 80225

ATTN:

D. H. Appel

G. L. Ducret

L. R. Hayes

W. R. Keefer

J. M. LaMonaca

J. S. Stuckless

U.S. Geological Survey

Federal Building, Room 224

Carson City, NV 89701

ATTN: P. A. Glancy

3 U.S. Geological Survey

101 Convention Center Drive

Suite 860 MS 509

Las Vegas, NV 89109

ATTN: R. W. Craig

D. Hoxie

D. Zesiger

U.S. Geological Survey

Water Resources Division

6770 S. Paradise Road

Las Vegas, NV 89119

ATTN: D. A. Beck

U.S. Geological Survey

P.O. Box 327, MS 721

Mercury, NV 89023

ATTN: A. L. Flint

2 U.S. Geological Survey

106 National Center

12201 Sunrise Valley Drive

Reston, VA 22092

ATTN: J. F. Devine

V. R. Schneider
No. of

Copies

4 U.S. Nuclear Regulatory Commission

Division of Engineering Safety

Washington, D.C. 20555

ATTN: D. Codell

S. Coplan

N. Eisenberg

NLS-260

T. J. Nicholson NL-005

U. S. Nuclear Regulatory Commission Division of Waste Management

Repository Licensing \& Quality Assurance-Project Directorate Washington, DC 20555

U. S. Nuclear Regulatory Commission Senior Project Manager

Repository Project Branch

Washington, DC 20555

Ellis D. Verink

Nuclear Waste Technical Review Board 4401 N.W. 18th Place

Gainesville, FL 32605

J. E. Watson, Jr.

Department of Environmental Sciences and Engineering

Campus Box 7400

University of North Carolina at Chapel Hill

Chapel Hill, NC 27599-7400

P. J. Weeden

U.S. Environmental Protection Agency

Nuclear Radiation Assessment Division

Environmental Monitoring Systems Laboratory

P.O. Box 93478

Las Vegas, NV 89193-3478

C. Whipple

Clement International

160 Spear Street, Suite 1380

San Francisco, CA 94105-1535 
No. of

Copies

P. J. Wierenga

New Mexico State University

Department of Soil Science

Las Cruces, NM 88004

R. Williams, Jr.

P.O. Box 10

Austin, NV 89310

\section{ONSITE}

2 DOE Field Office, Richland
D. C. Langstaff
J. J. Sutey
A5-90
A5-90

99 Pacific Northwest Laboratory

M. P. Bergeron

K6-77

J. W. Cary

K6-77

C. R. Cole

K6-77

J. L. DeVary

L. A. Doremus

K6-77

K6-96

D. W. Engel

D. J. Epstein

P. W. Eslinger (5)

M. J. Fayer

M. D. Freshley

T. R. Ginn

G. W. Gee

C. T. Kincaid

R. R. Kirkham

M. R. Kreiter

R. L. Lenhard

M. I. McKinley

P. D. Meyer

W. E. Nichols (25)

D. A. Perez

W. A. Perkins

J. C. Ritter

K. R. Roberson

J. S. Roberts

M. L. Rockhold

C. Sato

K7-34

K6-77

K6-96

K6-77

K6-77

K6-77

K6-77

K6-77

K6-77

K7-90

K6-77

K6-77

K6-77

K6-77

K6-86

K6-77

K6-77

K6-77

K7-15

K6-77

K6-77

T.D. Scheibe

K6-77

C. S. Simmons
No. of

Copies

J. L. Smoot

G. P. Streile

J. L. Stroh

J. E. Szecsody

L. V. Vail

R. W. Wallace

G. Whelan

M. D. White (25)

M. D. Williams

B. D. Wood

S. K. Wurstner

S. B. Yabusaki

Publishing Coordination

Technical Report Files (5)

K6-77

K6-77

K6-77

K6-77

K6-77

K6-77

K6-77

K6-77

K6-77

K6-77

K6-77

K6-77

Routing

R. M. Ecker

M. J. Graham

C. J. Hostetler

P. M. Irving

R. L. Skaggs

C. S. Sloane

P. C. Hays (last)
SEQUIM

K6-78

K6-81

K6-98

K6-77

K6-04

K6-86 